\title{
Mechanistic Investigation of Palladium-Catalyzed Meta-C-H Bond Activation of Arenes with Carboxyl Directing Group
}

Huiling Li, Miao Yang, Liyuan Jin, Yun-Fang Yang*, and Yuan-Bin She*.

College of Chemical Engineering, Zhejiang University of Technology, Hangzhou, Zhejiang 310014, China

*E-mail: yangyf@zjut.edu.cn

*E-mail: sheyb@zjut.edu.cn

\section{Contents}

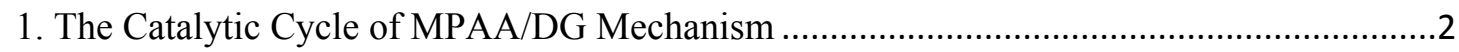

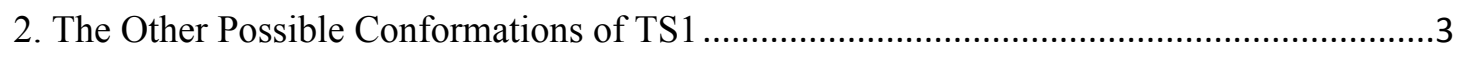

3. C-H Bond Activation TSs of the Pd/Ag Bimetallic Mechanism..........................................

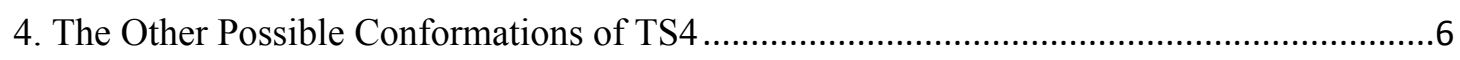

5. Activation of the ortho $_{2}-\mathrm{C}-\mathrm{H}$ Bond for Different Models...............................................

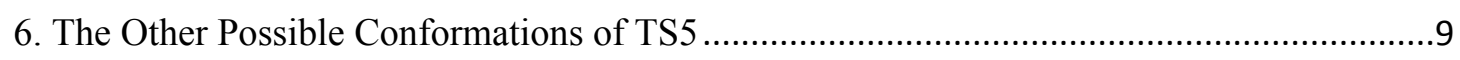

7. C-H Bond Activation TSs of the $\mathrm{Pd}(\mathrm{OAc})_{2}$ Mechanism.................................................11

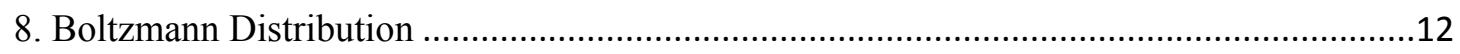

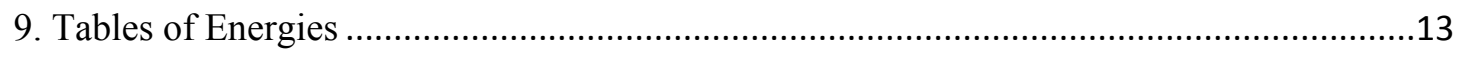

10. Cartesian Coordinates for Calculated Species .................................................................19 


\section{The Catalytic Cycle of MPAA/DG Mechanism}

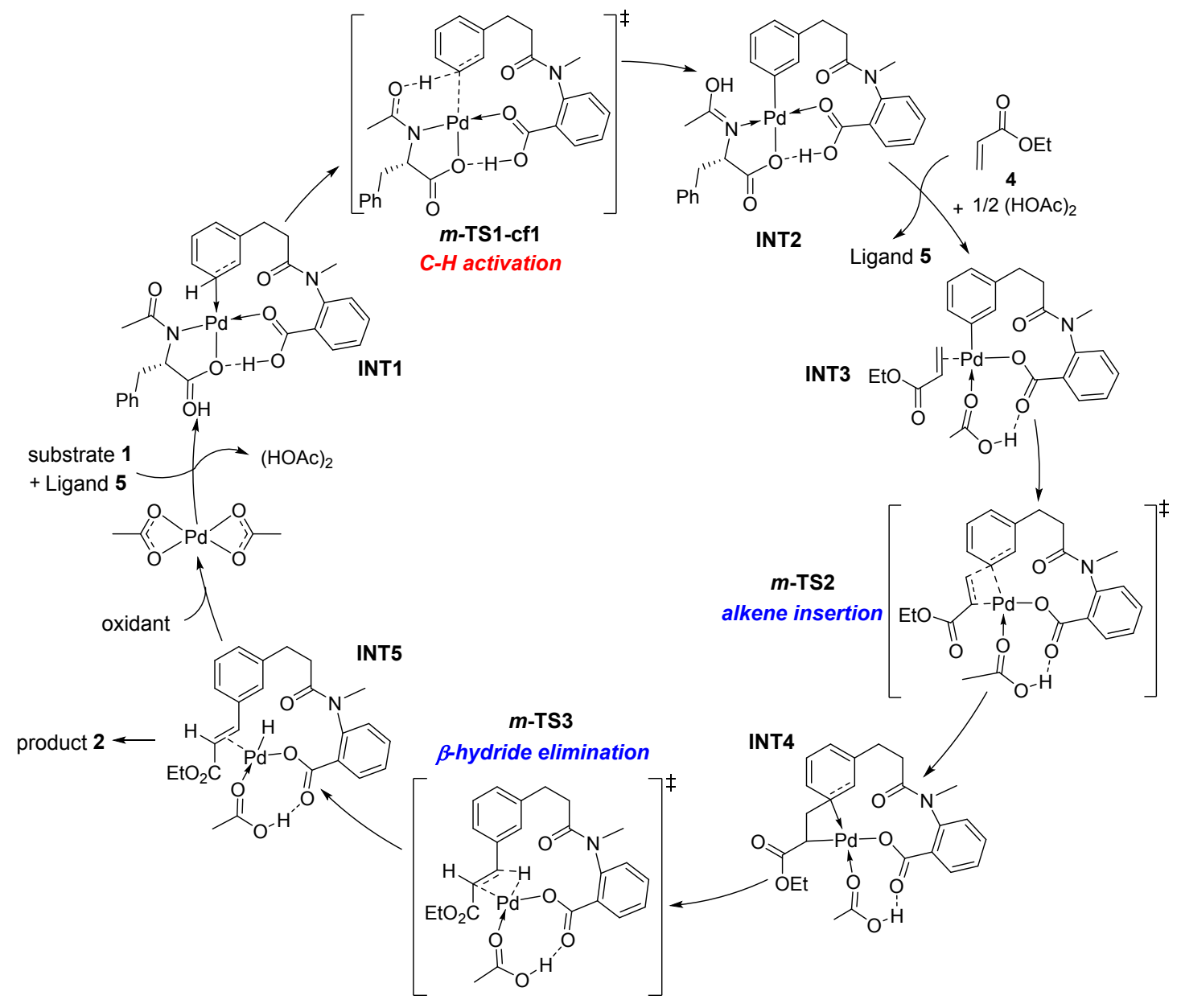

Figure S1. The catalytic cycle of $\mathrm{Pd}(\mathrm{OAc})_{2}$-catalyzed meta-C-H bond olefination. 


\section{The Other Possible Conformations of TS1}

Locating all conformations of the template is critical. This template is very flexible and there are many conformational minima which can be easily reached by torsional motions. The five dihedral angles $\psi 1-\psi 5$ were rotated with steps of $30^{\circ}$ manually. However, only several conformations are accessible for matching the geometry of the $\mathrm{C}-\mathrm{H}$ bond activation transition states, and the conformations with severe steric clash or not forming the macrocycle were discarded manually. There are only eight conformations for meta- and ortho $_{1}-\mathrm{C}-\mathrm{H}$ bond activation transition states and four conformations for para- $\mathrm{C}-\mathrm{H}$ bond activation transition state.

a)
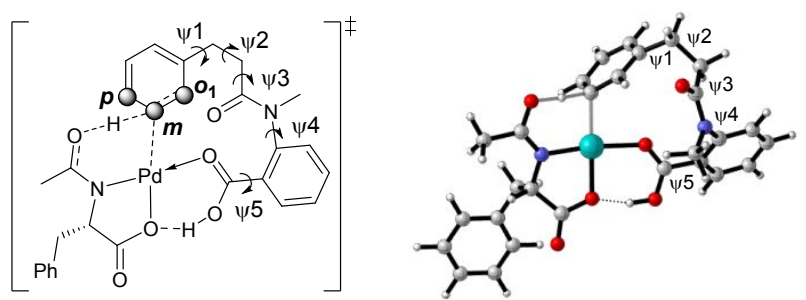

b)
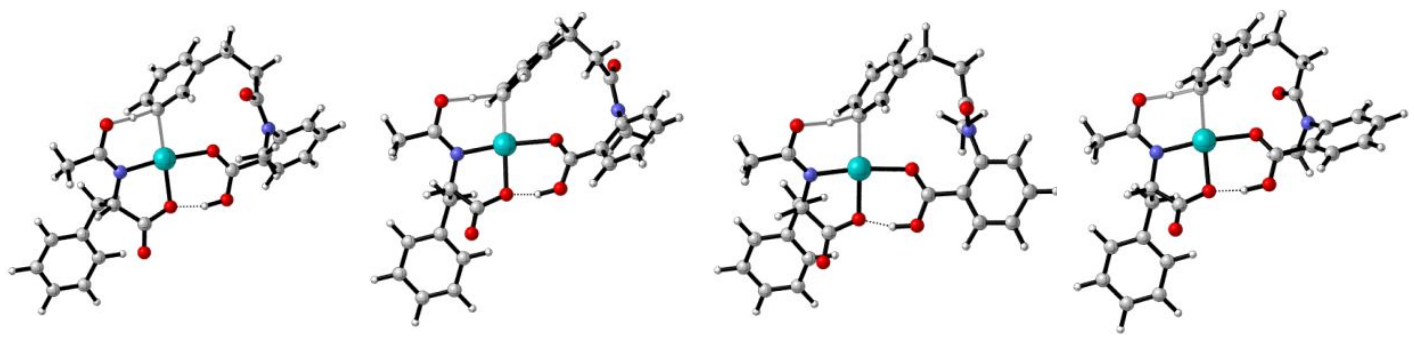

$m-\mathrm{TS} 1-\mathrm{cf} 1$
$\Delta \mathrm{G}_{\mathrm{sol}}^{\ddagger}=23.0 \mathrm{kcal} / \mathrm{mol}$

$$
\begin{gathered}
m-\mathrm{TS} 1-\mathrm{cf} 2 \\
\Delta \mathrm{G}_{\mathrm{sol}}^{\ddagger}=23.0 \mathrm{kcal} / \mathrm{mol}
\end{gathered}
$$
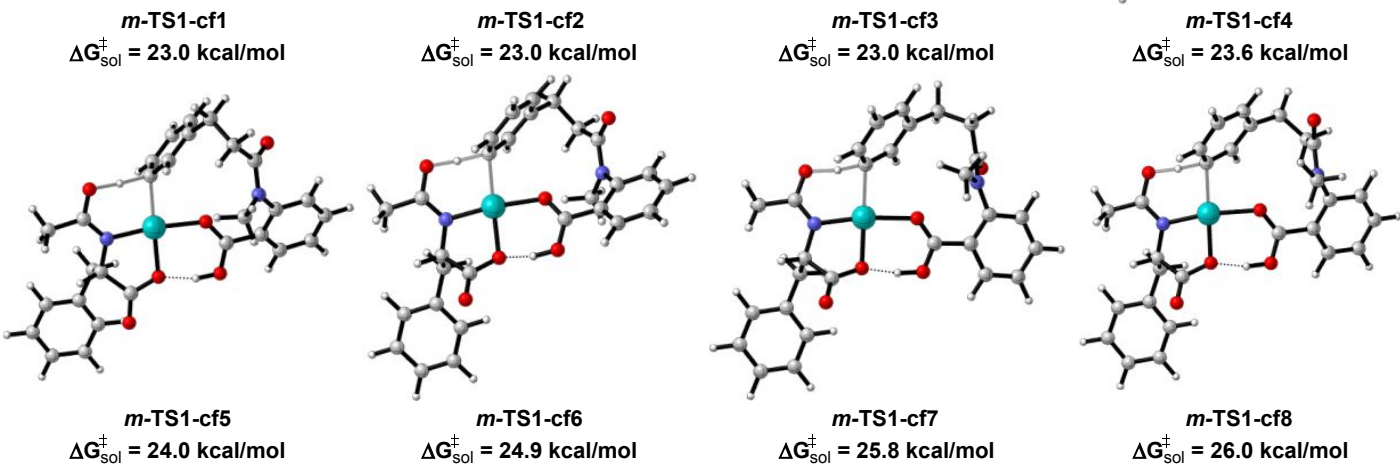

Figure S2. (a) Conformational search of $\mathrm{C}-\mathrm{H}$ activation TS of the cooperative MPAA/DG mechanism. (b) Optimized geometries of meta-C-H bond activation TSs of the cooperative MPAA/DG mechanism. 

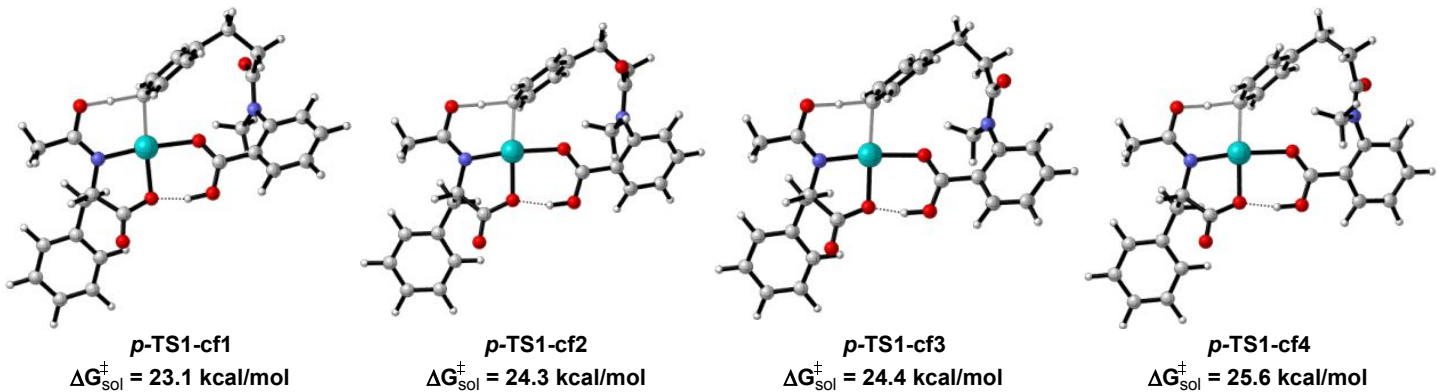

Figure S3. Optimized geometries of para-C-H bond activation TSs of the cooperative MPAA/DG mechanism.
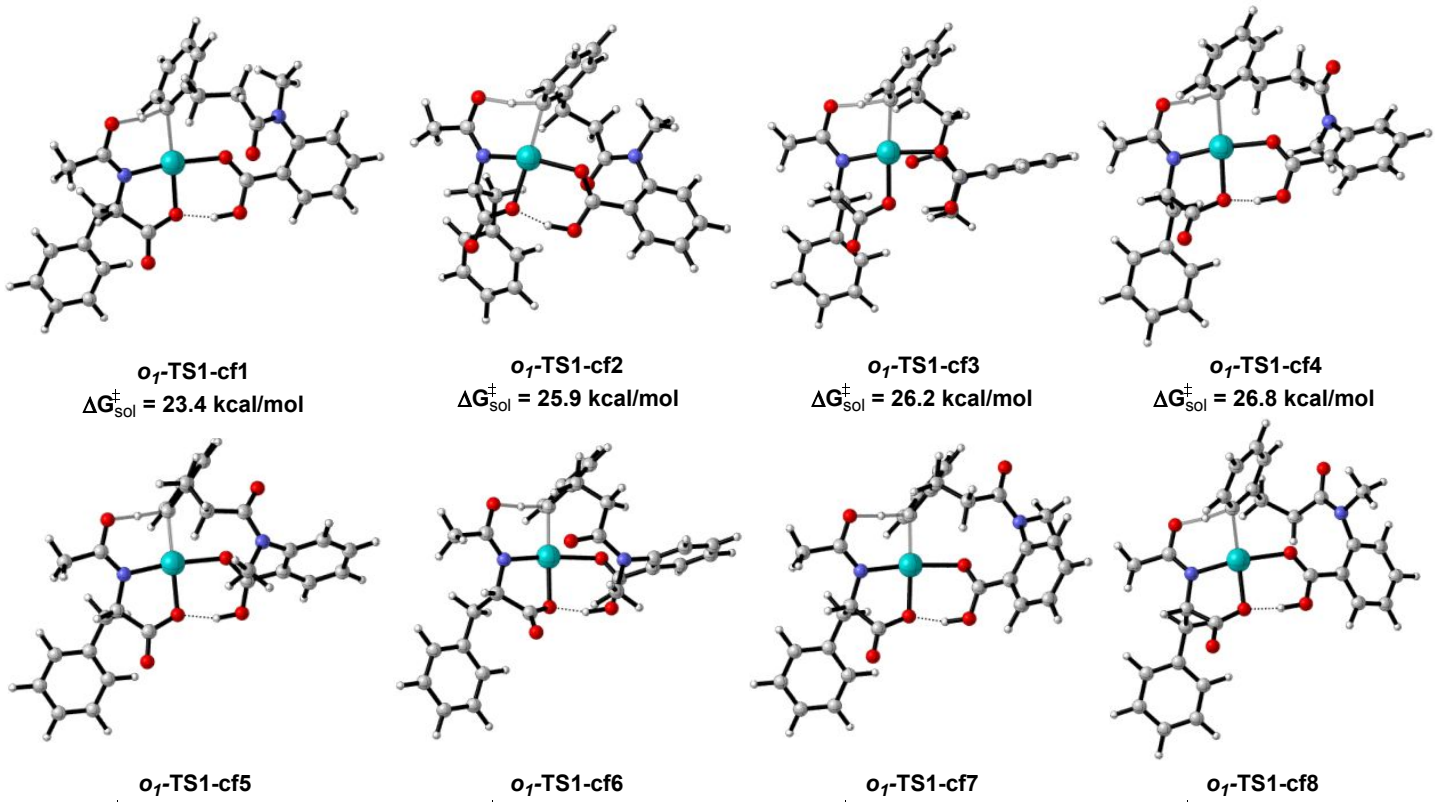

$\Delta G_{\text {sol }}^{\ddagger}=27.0 \mathrm{kcal} / \mathrm{mol}$

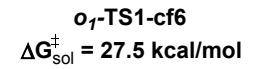

$\Delta \mathrm{G}_{\mathrm{sol}}^{\ddagger}=29.2 \mathrm{kcal} / \mathrm{mol}$

$\Delta G_{\text {sol }}^{\ddagger}=32.0 \mathrm{kcal} / \mathrm{mol}$

Figure S4. Optimized geometries of ortho ${ }_{1}-\mathrm{C}-\mathrm{H}$ bond activation $\mathrm{TSs}$ of the cooperative MPAA/DG mechanism. 


\section{C-H Bond Activation TSs of the Pd/Ag Bimetallic Mechanism}
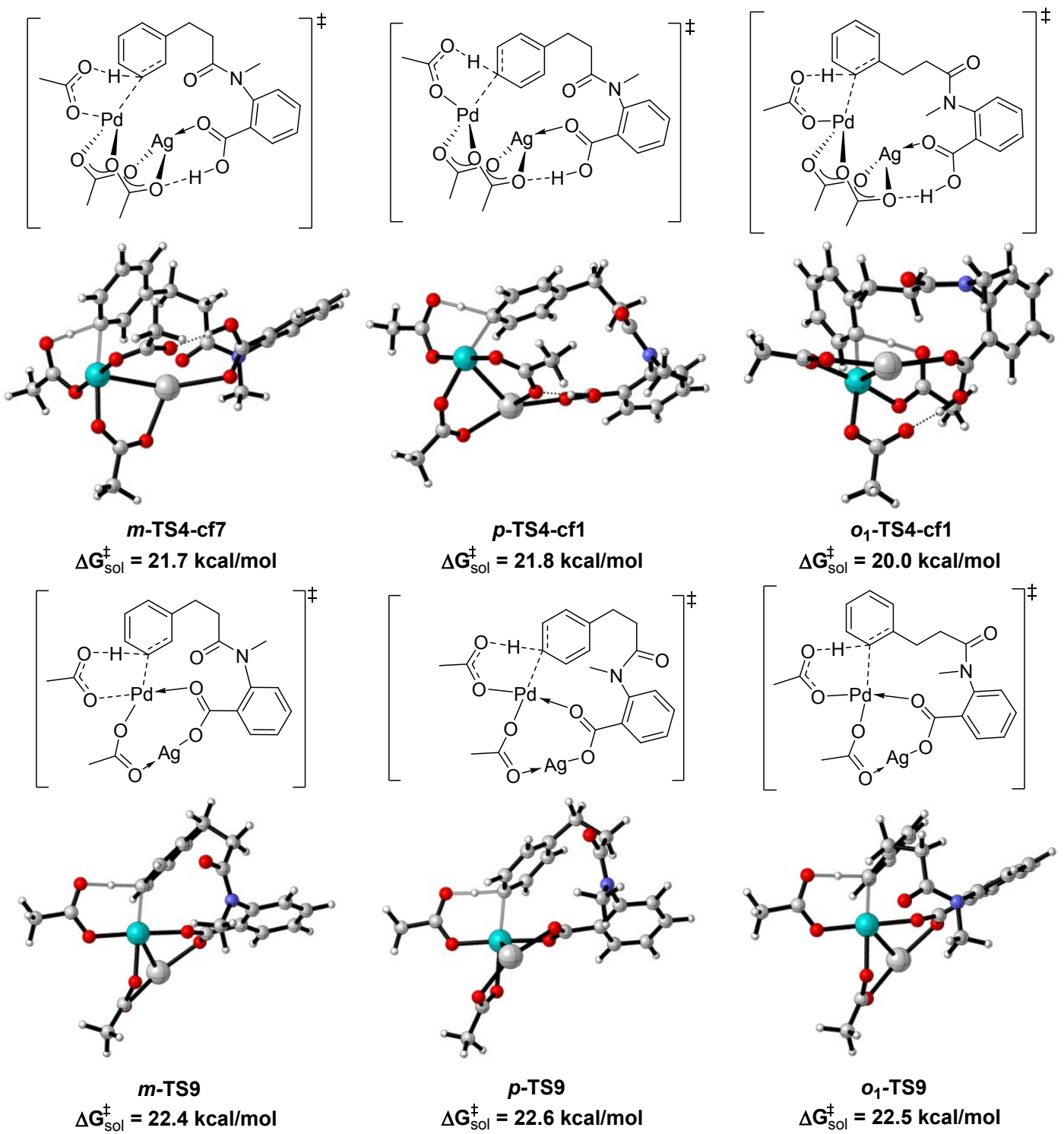

Figure S5. Optimized geometries of $\mathrm{C}-\mathrm{H}$ bond activation TSs of the PdAg-heterodimer mechanism. All energies are with respect to $\mathrm{Pd}_{3}(\mathrm{OAc})_{6}$ and $\mathrm{Ag}_{2}(\mathrm{OAc})_{2}$. 


\section{The Other Possible Conformations of TS4}

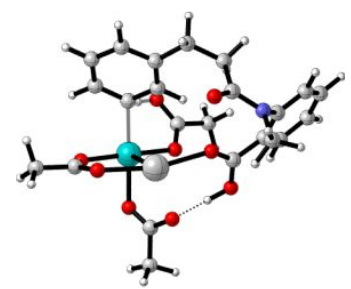

$m$-TS4-cf 1

$\Delta \mathrm{G}_{\mathrm{sol}}^{\ddagger}=22.5 \mathrm{kcal} / \mathrm{mol}$

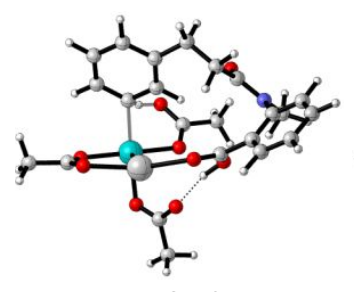

$m$-TS4-cf5

$\Delta \mathrm{G}_{\mathrm{sol}}^{\ddagger}=25.1 \mathrm{kcal} / \mathrm{mol}$

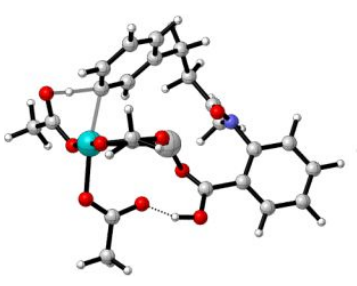

$m$-TS4-cf9

$\Delta \mathrm{G}_{\mathrm{sol}}^{\ddagger}=26.6 \mathrm{kcal} / \mathrm{mol}$

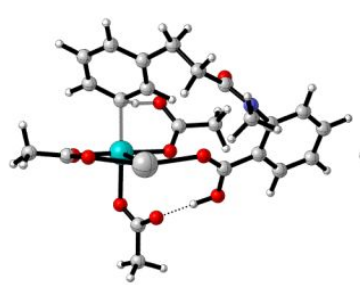

$m-\mathrm{TS} 4-\mathrm{cf} 13$
$\Delta \mathrm{G}_{\mathrm{sol}}^{\ddagger}=23.4 \mathrm{kcal} / \mathrm{mol}$

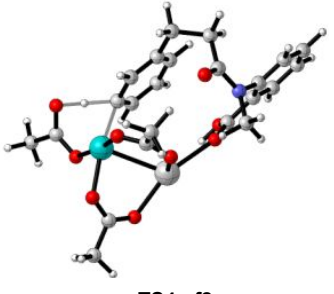

$m$-TS4-cf2

$\Delta G_{\mathrm{sol}}^{\ddagger}=31.5 \mathrm{kcal} / \mathrm{mol}$

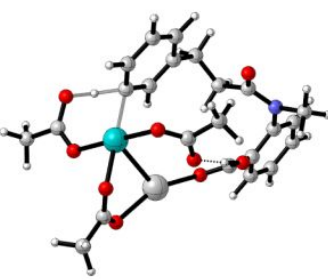

$m$-TS4-cf3

$\Delta G_{\text {sol }}^{\ddagger}=23.2 \mathrm{kcal} / \mathrm{mol}$

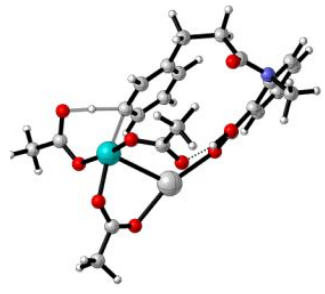

m-TS4-cf4

$\Delta G_{\mathrm{sol}}^{\ddagger}=25.2 \mathrm{kcal} / \mathrm{mol}$

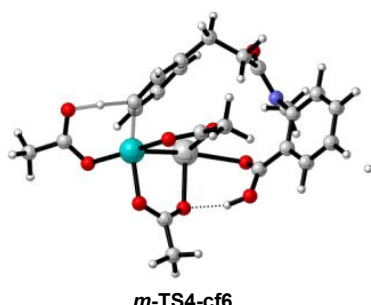

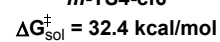

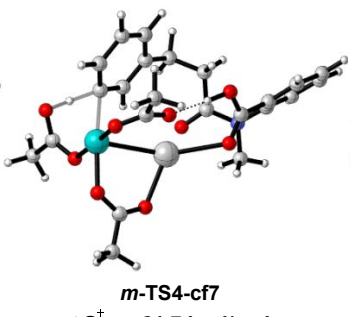

$\Delta G_{\text {sol }}^{\ddagger}=\mathbf{2 1 . 7} \mathrm{kcal} / \mathrm{mol}$
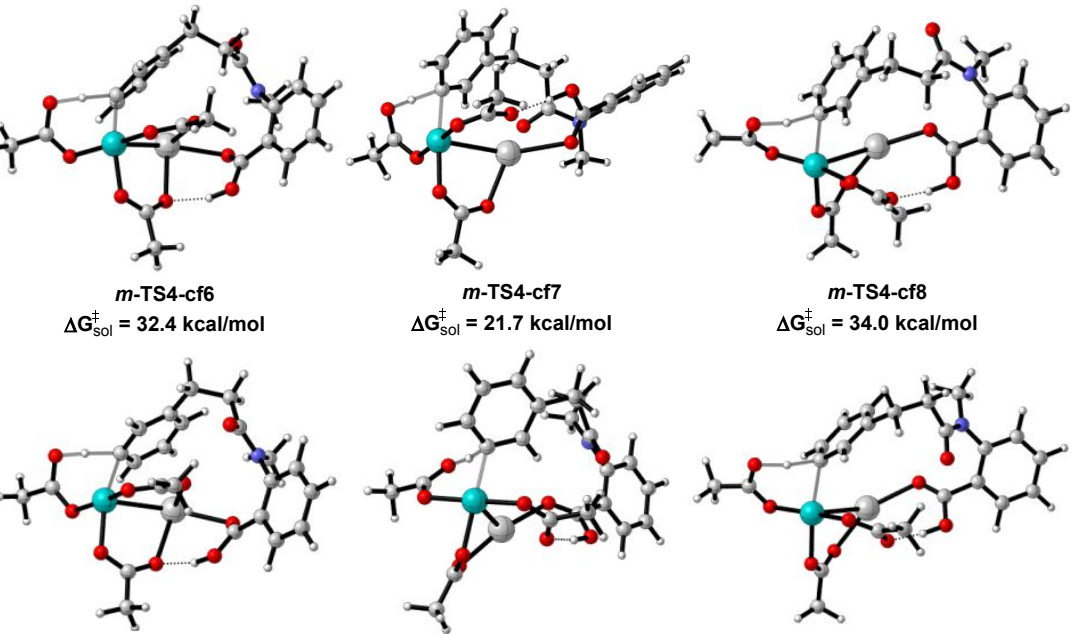

$m$-TS4-cf10

$\Delta G_{\text {sol }}^{\ddagger}=36.2 \mathrm{kcal} / \mathrm{mol}$

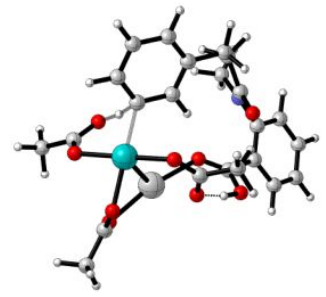

$m$-TS4-cf11

$\Delta G_{\text {sol }}^{\ddagger}=27.6 \mathrm{kcal} / \mathrm{mol}$

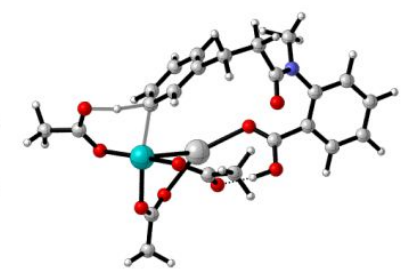

$m$-TS4-cf12

$\Delta G_{\text {sol }}^{\ddagger}=32.0 \mathrm{kcal} / \mathrm{mol}$

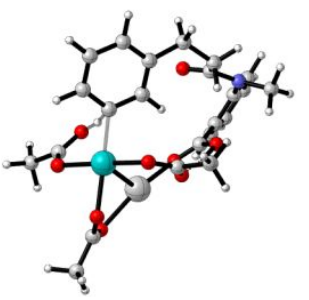

m-TS4-cf15

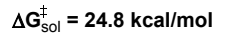

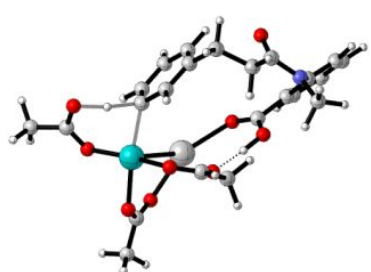

m-TS4-cf16 $\Delta G_{\text {sol }}^{\ddagger}=28.7 \mathrm{kcal} / \mathrm{mol}$

Figure S6. Optimized geometries of meta-C-H bond activation TSs of the PdAg-heterodimer mechanism. All energies are with respect to $\mathrm{Pd}_{3}(\mathrm{OAc})_{6}$ and $\mathrm{Ag}_{2}(\mathrm{OAc})_{2}$. 


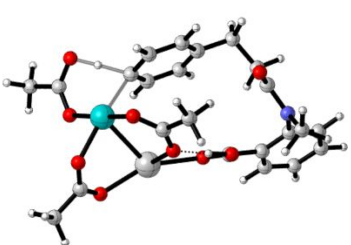

p-TS4-cf1

$\Delta G_{\text {sol }}^{\ddagger}=21.8 \mathrm{kcal} / \mathrm{mol}$

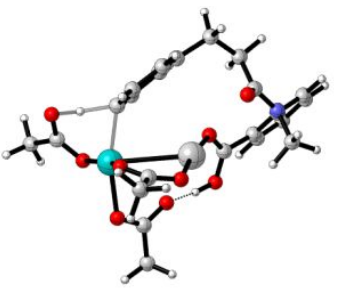

p-TS4-cf5

$\Delta \mathrm{G}_{\mathrm{sol}}^{\ddagger}=30.6 \mathrm{kcal} / \mathrm{mol}$

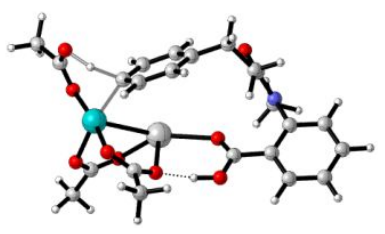

p-TS4-cf2

$\Delta G_{\text {sol }}^{\ddagger}=24.2 \mathrm{kcal} / \mathrm{mol}$

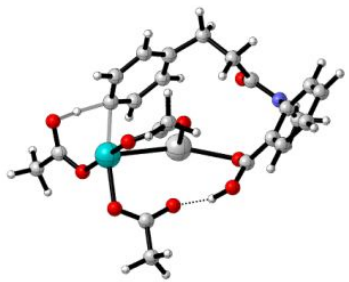

p-TS4-cf6

$\Delta \mathrm{G}_{\mathrm{sol}}^{\ddagger}=27.8 \mathrm{kcal} / \mathrm{mol}$

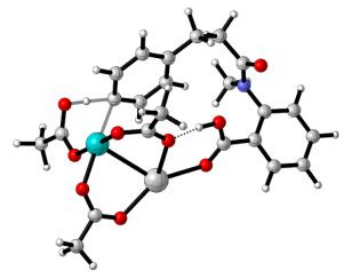

p-TS4-cf3

$\Delta G_{\text {sol }}^{\ddagger}=27.8 \mathrm{kcal} / \mathrm{mol}$

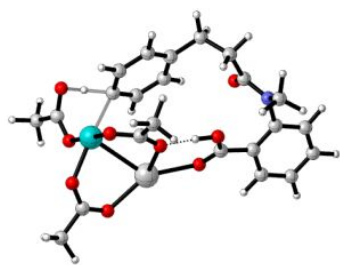

p-TS4-cf4

$\Delta G_{\text {sol }}^{\ddagger}=21.9 \mathrm{kcal} / \mathrm{mol}$

Figure S7. Optimized geometries of para-C-H bond activation TSs of the PdAg-heterodimer mechanism. All energies are with respect to $\mathrm{Pd}_{3}(\mathrm{OAc})_{6}$ and $\mathrm{Ag}_{2}(\mathrm{OAc})_{2}$.

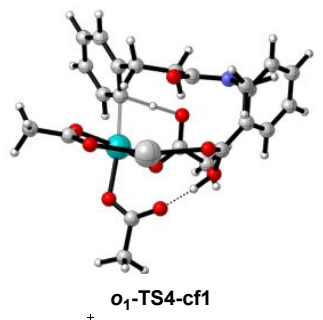

$\Delta G_{\text {sol }}^{\ddagger}=20.0 \mathrm{kcal} / \mathrm{mol}$

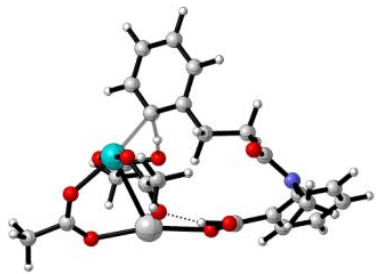

$0_{1}$-TS4-cf5

$\Delta G_{\text {sol }}^{\ddagger}=26.3 \mathrm{kcal} / \mathrm{mol}$

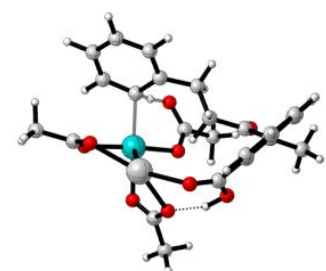

$\mathrm{O}_{1}$-TS4-cf2

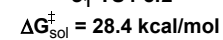

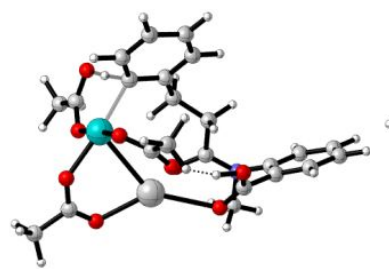

0 1 -TS4-cf6

$\Delta G_{\mathrm{sol}}^{\ddagger}=24.0 \mathrm{kcal} / \mathrm{mol}$

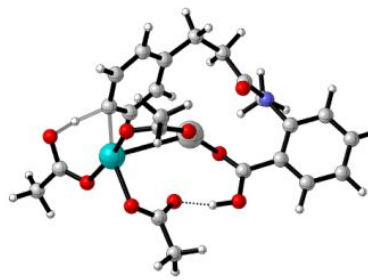

p-TS4-cf7

$p-$ TS4-cf7
$\Delta G_{\text {sol }}^{\ddagger}=32.6 \mathrm{kcal} / \mathrm{mol}$

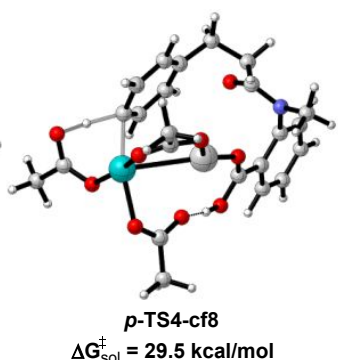

$\Delta \mathrm{G}_{\mathrm{sol}}^{\ddagger}=29.5 \mathrm{kcal} / \mathrm{mol}$

Figure S8. Optimized geometries of ortho $_{1}-\mathrm{C}-\mathrm{H}$ bond activation TSs of the PdAg-heterodimer mechanism. All energies are with respect to $\mathrm{Pd}_{3}(\mathrm{OAc})_{6}$ and $\mathrm{Ag}_{2}(\mathrm{OAc})_{2}$. 


\section{Activation of the ortho $2-\mathrm{C}-\mathrm{H}$ Bond for Different Models}
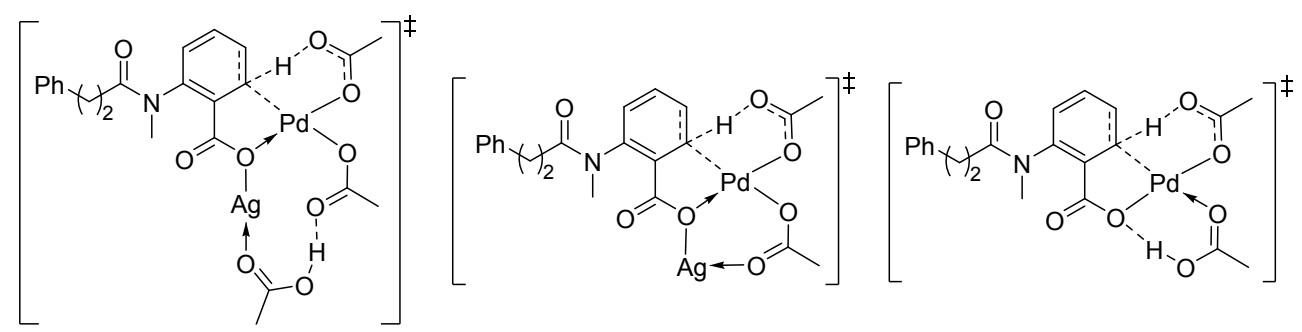

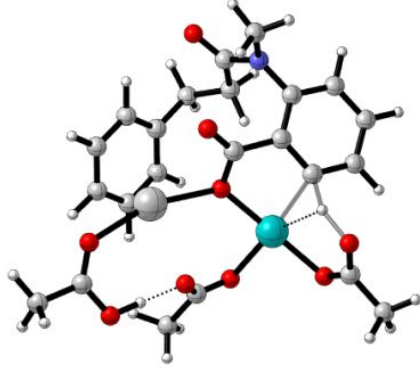

$\mathrm{O}_{2}$-TS1-a

$\Delta G_{\text {sol }}^{\ddagger}=25.9 \mathrm{kcal} / \mathrm{mol}$

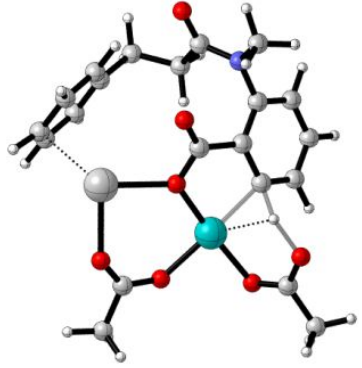

$o_{2}$-TS1-b

$\Delta G_{\text {sol }}^{\ddagger}=28.6 \mathrm{kcal} / \mathrm{mol}$

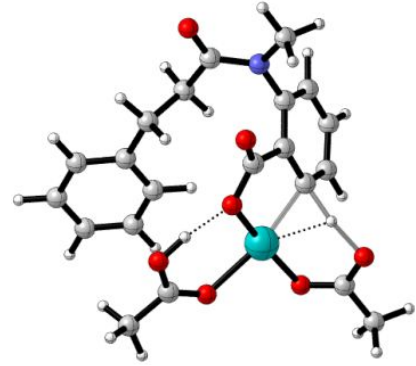

$\mathrm{O}_{2}$-TS1-C

$\Delta G^{\ddagger}=31.8 \mathrm{kcal} / \mathrm{mol}$

Figure S9. The possible ortho $_{2}-\mathrm{C}-\mathrm{H}$ bond activation mechanistic models.
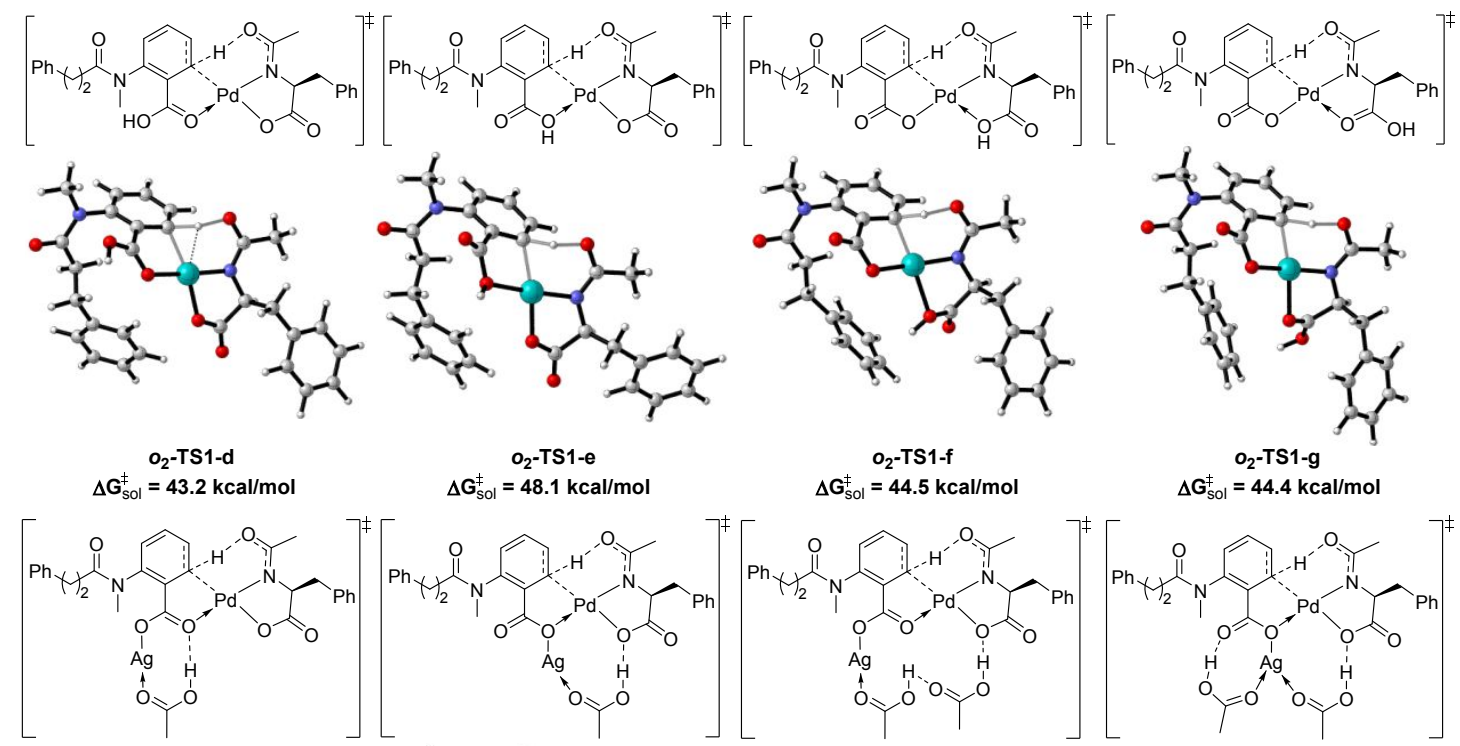

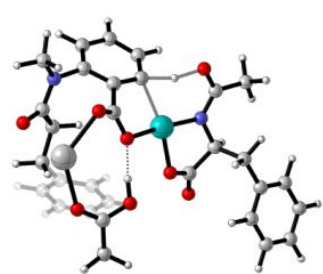

o - TS1-h

$\Delta G_{\text {sol }}^{\ddagger}=\mathbf{4 0 . 3} \mathrm{kcal} / \mathrm{mol}$

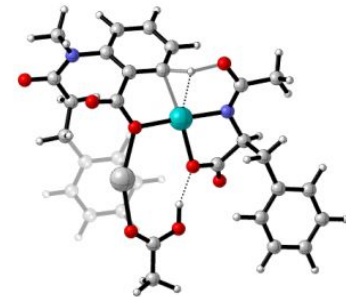

$\mathrm{O}_{2}$-TS1-i

$\Delta G_{\text {sol }}^{\ddagger}=32.3 \mathrm{kcal} / \mathrm{mol}$

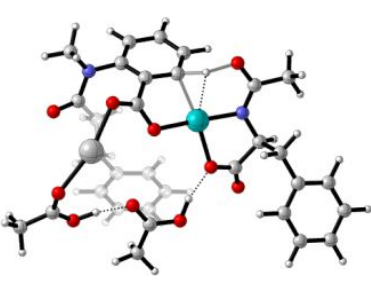

$\mathrm{o}_{2}$-TS1-j

$\Delta G_{\text {sol }}^{\ddagger}=37.7 \mathrm{kcal} / \mathrm{mol}$

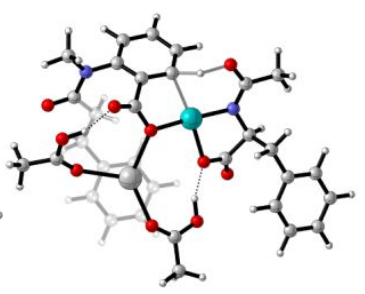

$\mathrm{o}_{2}$-TS1-k $\Delta G_{\text {sol }}^{\ddagger}=33.2 \mathrm{kcal} / \mathrm{mo}$

Figure S10. The possible ortho $_{2}-\mathrm{C}-\mathrm{H}$ bond activation mechanistic models when MPAA was involved. 


\section{The Other Possible Conformations of TS5}
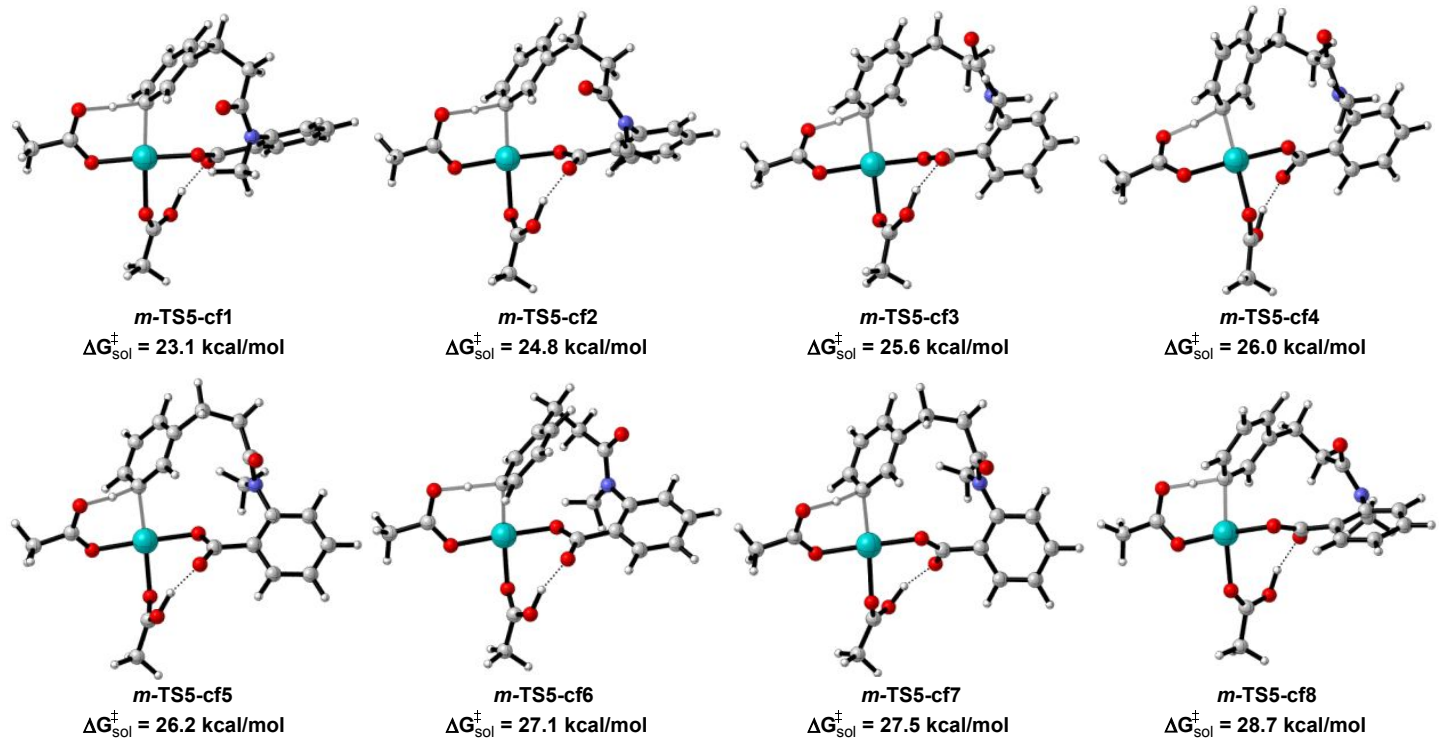

Figure S11. Optimized geometries of meta-C-H bond activation TSs of the $\mathrm{Pd}(\mathrm{OAc})_{2}$ mechanism with DG in $\kappa^{1}$ coordination.

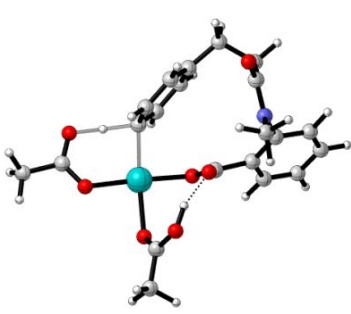

p-TS5-cf1

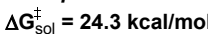

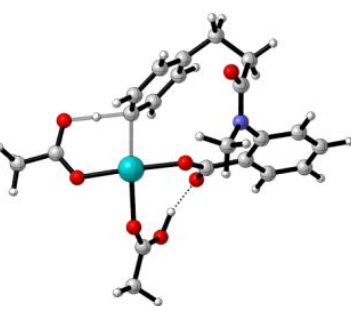

$p-\mathrm{TS} 5-\mathrm{cf} 2$
$\Delta \mathrm{G}_{\mathrm{sol}}^{\ddagger}=24.9 \mathrm{kcal} / \mathrm{mol}$

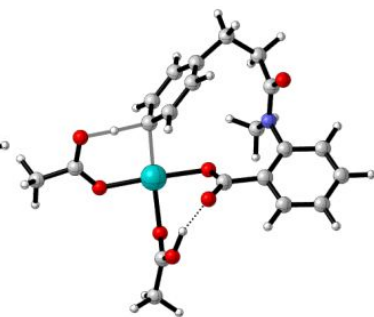

p-TS5-cf3

$\Delta \mathrm{G}_{\mathrm{sol}}^{\ddagger}=27.3 \mathrm{kcal} / \mathrm{mol}$

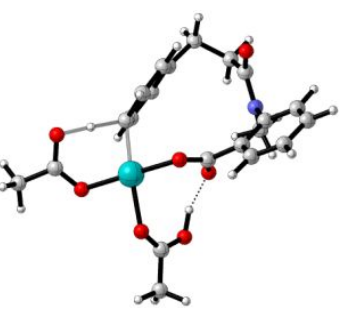

p-TS5-cf4 $\Delta G_{\text {sol }}^{\ddagger}=28.7 \mathrm{kcal} / \mathrm{mol}$

Figure S12. Optimized geometries of para-C-H bond activation TSs of the $\mathrm{Pd}(\mathrm{OAc})_{2}$ mechanism with DG in $\kappa^{1}$ coordination.

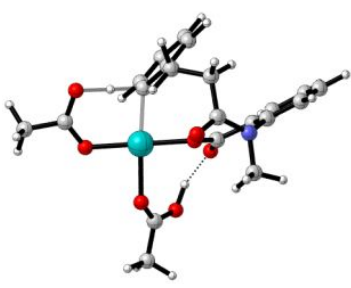

$0_{1}$-TS5-cf1

$\Delta \mathbf{G}_{\mathrm{sol}}^{\ddagger}=24.2 \mathrm{kcal} / \mathrm{mol}$

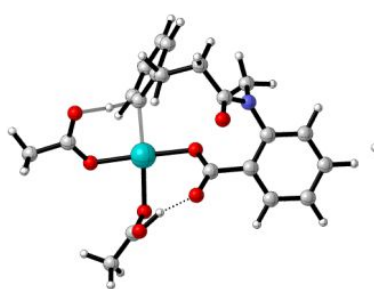

$0_{1}$-TS5-cf5

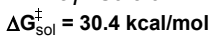
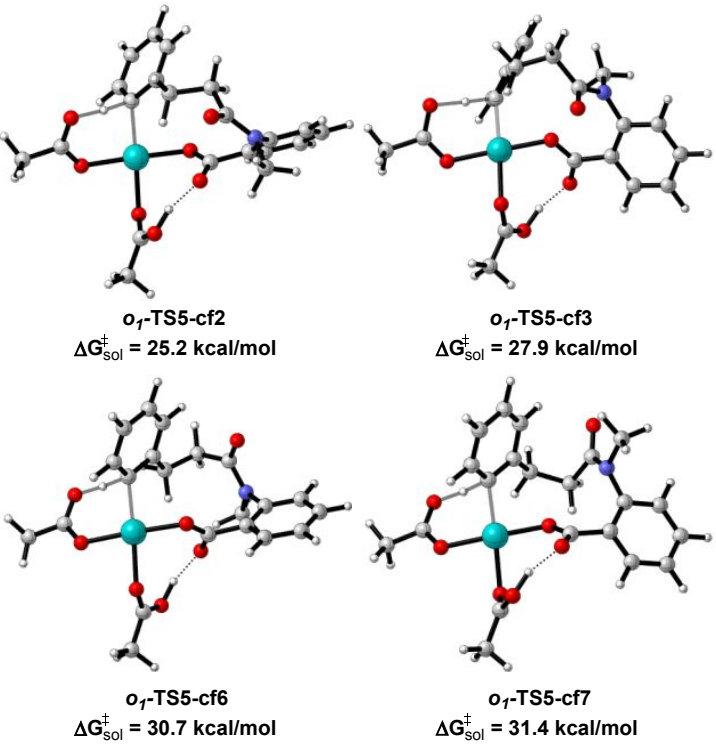

01-TS5-cf7

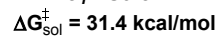

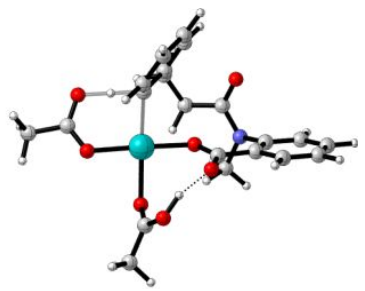

$0_{1}$-TS5-cf 4

$\Delta \mathrm{G}_{\mathrm{sol}}^{\ddagger}=28.3 \mathrm{kcal} / \mathrm{mol}$

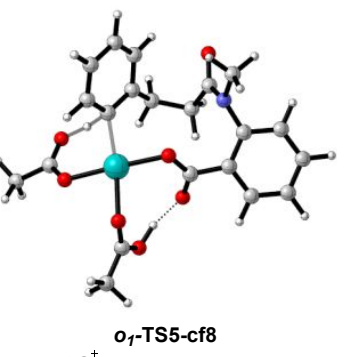

$\Delta G_{\text {sol }}^{\ddagger}=35.7 \mathrm{kcal} / \mathrm{mol}$

Figure S13. Optimized geometries of ortho ${ }_{1}-\mathrm{C}-\mathrm{H}$ bond activation $\mathrm{TSs}$ of the $\mathrm{Pd}(\mathrm{OAc})_{2}$ mechanism with DG in $\kappa^{1}$ coordination. 


\section{C-H Bond Activation TSs of the $\operatorname{Pd}(\mathrm{OAc})_{2}$ Mechanism}

The $\mathrm{C}-\mathrm{H}$ bond activation TSs with proton either on the DG or on the coordinated carboxylic acid group were studied. The meta-C-H bond activation with proton on the coordinated carboxylic acid group (m-TS5-cf1) require a barrier of $23.1 \mathrm{kcal} / \mathrm{mol}$, while the meta-C-H bond activation with proton on the DG $(\boldsymbol{m}$-TS10) require a barrier of $30.7 \mathrm{kcal} / \mathrm{mol}$ which is much higher than $\boldsymbol{m}$-TS5-cf1. The $p \mathrm{~K}_{\mathrm{a}}$ of acetic acid is larger than the $p \mathrm{~K}_{\mathrm{a}}$ of benzoic acid. Therefore the proton staying on the acetic acid is favorable than on the DG.
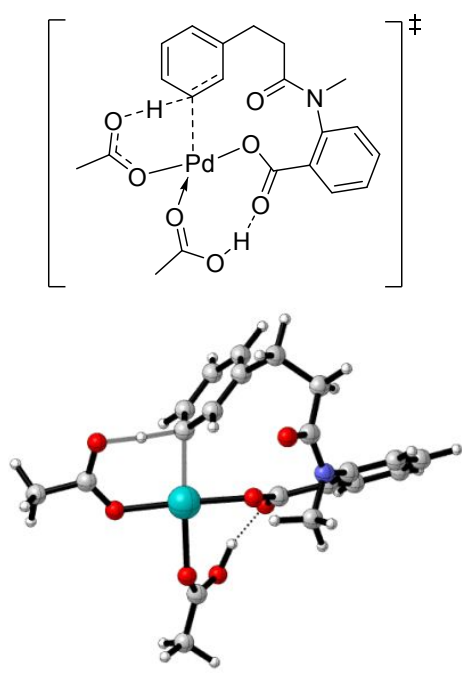

$m$-TS5-cf1

$\Delta G_{\text {sol }}^{\ddagger}=23.1 \mathrm{kcal} / \mathrm{mol}$
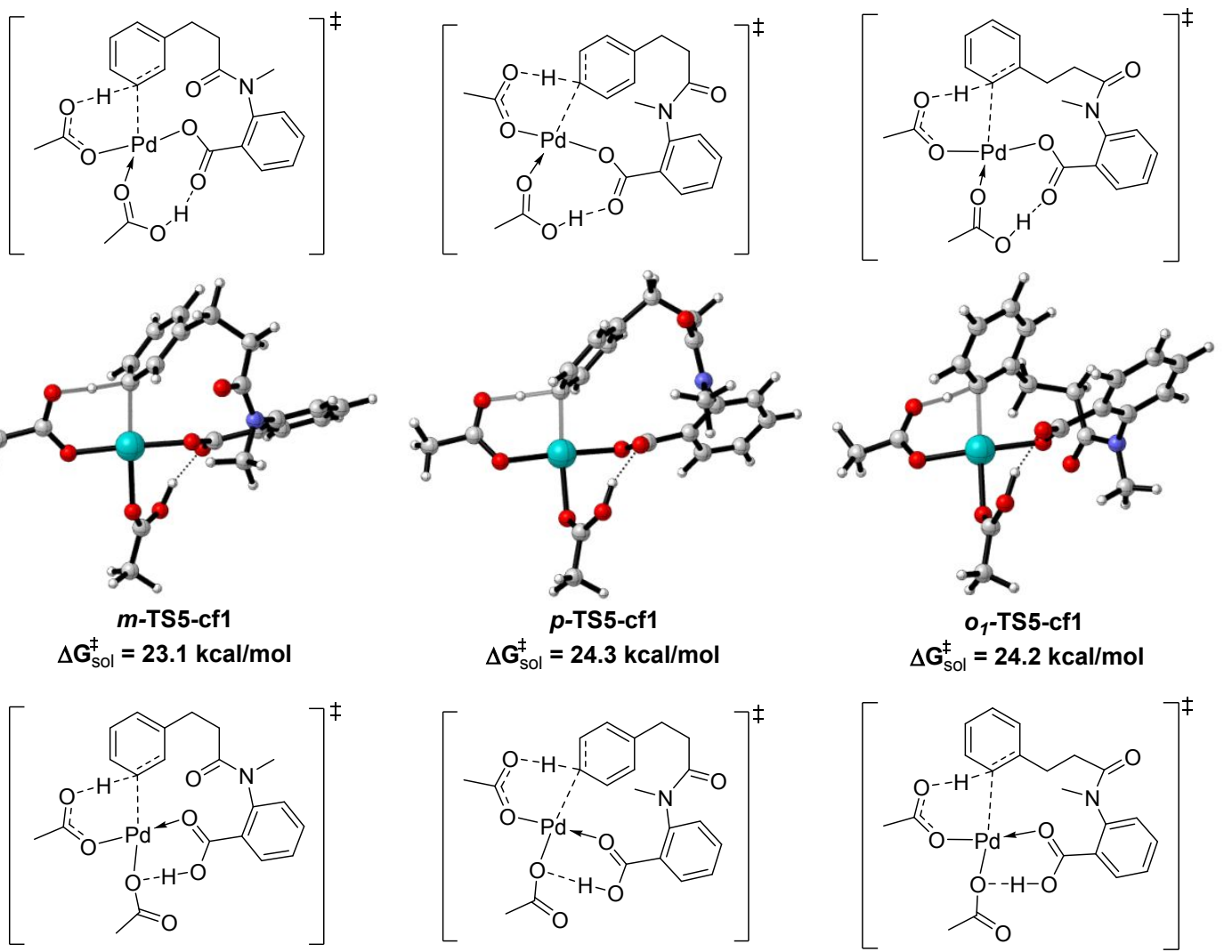

$\Delta \mathrm{G}_{\mathrm{sol}}^{\ddagger}=24.3 \mathrm{kcal} / \mathrm{mol}$

$0_{1}$-TS5-cf1

$\Delta \mathrm{G}_{\mathrm{sol}}^{\ddagger}=24.2 \mathrm{kcal} / \mathrm{mol}$
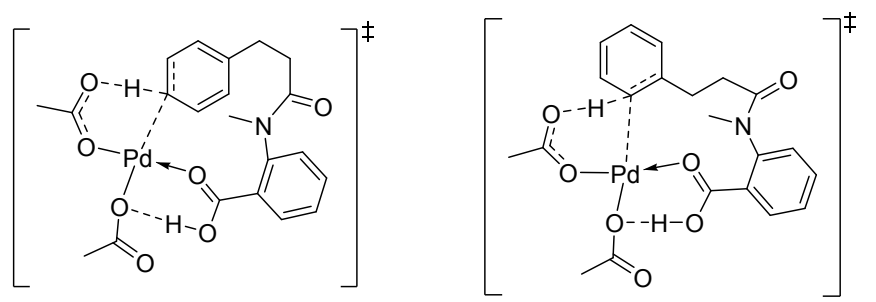

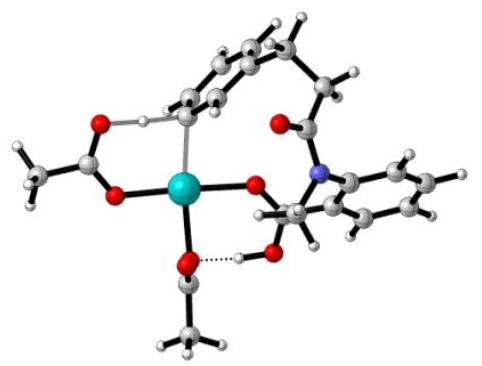

$m-$ TS10

$\Delta G_{\text {sol }}^{\ddagger}=\mathbf{3 0 . 7} \mathrm{kcal} / \mathrm{mol}$

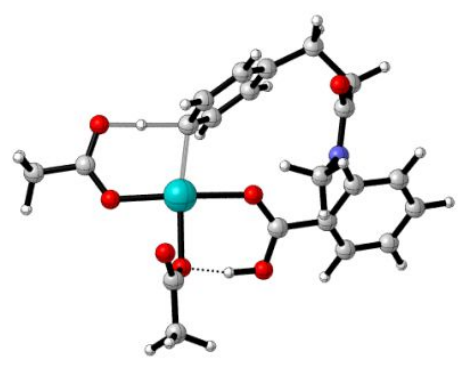

p-TS10

$\Delta G_{\text {sol }}^{\ddagger}=31.1 \mathrm{kcal} / \mathrm{mol}$

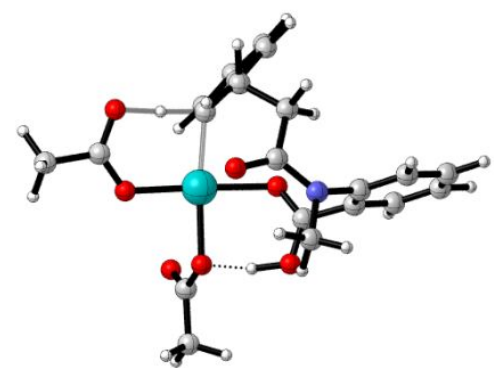

$0_{1}-\mathrm{TS} 10$

$\Delta \mathrm{G}_{\mathrm{sol}}^{\ddagger}=33.2 \mathrm{kcal} / \mathrm{mol}$

Figure S14. Optimized geometries of $\mathrm{C}-\mathrm{H}$ bond activation $\mathrm{TSs}$ of the $\mathrm{Pd}(\mathrm{OAc})_{2}$ mechanism with DG in $\kappa^{1}$ coordination. 


\section{Boltzmann Distribution}

Conformations within $3 \mathrm{kcal} / \mathrm{mol}$ of the global minimum are included in the Boltzmann distribution and are reported in the supporting information below. Theoretical regioselectivities were computed using a Boltzmann distribution at $298 \mathrm{~K}$ $\left(25^{\circ} \mathrm{C}\right)$. The probability $\left(P_{i}\right)$ of reaction through each transition state was computed as:

$$
P_{i}=\frac{e^{-\Delta G_{i}^{\ddagger} / R T}}{\sum_{i=1}^{N} e^{-\Delta G_{i}^{\ddagger} / R T}}
$$

The probabilities for all transition states leading to a particular regioisomer were summed to compute a theoretical regioselectivity. In most cases, there are several low-energy conformations, so the theoretical regioselectivity could not be approximated using a single lowest-energy transition state.

\begin{tabular}{|c|c|c|c|c|}
\hline & Transition State & $\Delta G^{\ddagger}$ & $\Delta \Delta G^{\ddagger}$ & $\%$ \\
\hline \multirow[t]{14}{*}{ TS1 Pd-Gly } & $m$-TS1-cf1 & 23.0 & 0.0 & 21.5 \\
\hline & $m$-TS1-cf2 & 23.0 & 0.0 & 21.5 \\
\hline & $m$-TS1-cf3 & 23.0 & 0.0 & 21.5 \\
\hline & $m$-TS1-cf4 & 23.6 & 0.6 & 7.8 \\
\hline & $m$-TS1-cf5 & 24.0 & 1.0 & 4.0 \\
\hline & $m$-TS1-cf6 & 24.9 & 1.9 & 0.9 \\
\hline & $m$-TS1-cf7 & 25.8 & 2.8 & 0.2 \\
\hline & $m$-TS1-cf8 & 26.0 & 3.0 & 0.1 \\
\hline & $p$-TS1-cf1 & 23.1 & 0.1 & 9.1 \\
\hline & $p$-TS1-cf2 & 24.3 & 1.3 & 1.2 \\
\hline & $p$-TS1-cf3 & 24.4 & 1.4 & 1.0 \\
\hline & $p$-TS1-cf4 & 25.6 & 2.6 & 0.1 \\
\hline & $o_{1}$-TS1-cf1 & 23.4 & 0.4 & 10.9 \\
\hline & $o_{1}$-TS1-cf2 & 25.9 & 2.9 & 0.2 \\
\hline & Transition State & $\Delta G^{t}$ & $\Delta \Delta G^{\ddagger}$ & $\%$ \\
\hline \multirow[t]{8}{*}{ TS5 Pd(OAc) $)_{2}$} & $m$-TS5-cf1 & 23.1 & 0.0 & 73.9 \\
\hline & $m$-TS5-cf2 & 24.8 & 1.7 & 4.2 \\
\hline & $m$-TS5-cf3 & 25.6 & 2.5 & 1.1 \\
\hline & $m$-TS5-cf4 & 26.0 & 2.9 & 0.5 \\
\hline & $p$-TS5-cf1 & 24.3 & 1.2 & 4.9 \\
\hline & $p$-TS5-cf2 & 24.9 & 1.8 & 1.8 \\
\hline & $o_{1}$-TS5-cf1 & 24.2 & 1.1 & 11.5 \\
\hline & $o_{1}$-TS5-cf2 & 25.2 & 2.1 & 2.1 \\
\hline
\end{tabular}




\section{Tables of Energies}

Table S1. The Calculated Single-Point Solvation Energies of the Key Transition States Using Different Functionals

\begin{tabular}{|c|c|c|c|c|}
\hline & Transition State & $\begin{array}{c}\text { B3LYP-D3 } \\
\left(\Delta G^{\ddagger} / \mathbf{k c a l} / \mathbf{m o l}\right)\end{array}$ & $\begin{array}{c}\text { M06 } \\
\left(\Delta G^{\ddagger} / \mathbf{k c a l} / \mathbf{m o l}\right)\end{array}$ & $\begin{array}{c}\omega \mathrm{B} 97 \mathrm{X}-\mathrm{D} \\
\left(\Delta G^{\ddagger} / \mathrm{kcal} / \mathrm{mol}\right)\end{array}$ \\
\hline \multirow[t]{20}{*}{ Pd-MPAA } & $m$-TS1-cf1 & 23.0 & 22.2 & 24.0 \\
\hline & $m$-TS1-cf2 & 23.0 & 22.2 & 24.3 \\
\hline & $m$-TS1-cf3 & 23.0 & 22.1 & 24.0 \\
\hline & $m$-TS1-cf4 & 23.6 & 22.6 & 24.7 \\
\hline & $m$-TS1-cf5 & 24.0 & 22.8 & 24.8 \\
\hline & $m$-TS1-cf6 & 24.9 & 23.6 & 25.9 \\
\hline & $m$-TS1-cf7 & 25.8 & 24.9 & 27.2 \\
\hline & $m$-TS1-cf8 & 26.0 & 24.8 & 27.5 \\
\hline & $p$-TS1-cf1 & 23.1 & 21.8 & 24.3 \\
\hline & $p$-TS1-cf2 & 24.3 & 22.8 & 25.5 \\
\hline & $p$-TS1-cf3 & 24.4 & 23.0 & 25.5 \\
\hline & $p$-TS1-cf4 & 25.6 & 23.9 & 26.8 \\
\hline & $o_{1}$-TS1-cf1 & 23.4 & 22.4 & 23.9 \\
\hline & $o_{1}$-TS1-cf2 & 25.9 & 25.2 & 26.9 \\
\hline & $o_{1}$-TS1-cf3 & 26.2 & 25.6 & 26.7 \\
\hline & $o_{1}-\mathrm{TS} 1-\mathrm{cf} 4$ & 26.8 & 26.0 & 27.5 \\
\hline & $o_{1}$-TS1-cf5 & 27.0 & 24.7 & 26.9 \\
\hline & $o_{1}$-TS1-cf6 & 27.5 & 27.5 & 28.5 \\
\hline & $o_{1}$-TS1-cf7 & 29.2 & 28.7 & 30.4 \\
\hline & $o_{1}$-TS1-cf8 & 32.0 & 31.5 & 33.4 \\
\hline \multirow[t]{3}{*}{ Pd-Ag } & $m$-TS4-cf7 & 21.7 & 20.7 & 22.7 \\
\hline & $p$-TS4-cf1 & 21.8 & 20.6 & 23.1 \\
\hline & $o_{1}$-TS4-cf1 & 20.0 & 20.5 & 21.6 \\
\hline \multirow[t]{14}{*}{$\operatorname{Pd}(\mathrm{OAc})_{2}$} & $m$-TS5-cf1 & 23.1 & 22.9 & 24.9 \\
\hline & $m$-TS5-cf2 & 24.8 & 24.4 & 26.5 \\
\hline & $m$-TS5-cf3 & 25.6 & 24.4 & 26.8 \\
\hline & $m$-TS5-cf4 & 26.0 & 25.1 & 27.6 \\
\hline & $m$-TS5-cf5 & 26.2 & 25.2 & 27.3 \\
\hline & $m$-TS5-cf6 & 27.1 & 26.1 & 28.8 \\
\hline & $m$-TS5-cf7 & 27.5 & 26.6 & 28.6 \\
\hline & $m$-TS5-cf8 & 28.8 & 27.4 & 30.5 \\
\hline & $p$-TS5-cf1 & 24.3 & 23.0 & 25.7 \\
\hline & $p$-TS5-cf2 & 24.9 & 23.4 & 26.5 \\
\hline & $p$-TS5-cf3 & 27.3 & 25.7 & 28.5 \\
\hline & $p$-TS5-cf4 & 28.7 & 26.6 & 29.9 \\
\hline & $o_{1}$-TS5-cf1 & 24.2 & 22.8 & 25.2 \\
\hline & $o_{1}$-TS5-cf2 & 25.2 & 24.7 & 26.6 \\
\hline
\end{tabular}




\begin{tabular}{lllll}
\hline $\boldsymbol{o}_{1}$-TS5-cf3 & 27.9 & 27.6 & 29.1 \\
\hline $\boldsymbol{o}_{\mathbf{1}}$-TS5-cf4 & 28.3 & 27.6 & 29.8 \\
\hline $\boldsymbol{o}_{\mathbf{1}}$-TS5-cf5 & 30.4 & 29.3 & 31.6 \\
\hline $\boldsymbol{o}_{\mathbf{1}}$-TS5-cf6 & 30.7 & 29.6 & 31.7 \\
\hline $\boldsymbol{o}_{\mathbf{1}}$-TS5-cf7 & 31.4 & 31.4 & 33.0 \\
\hline $\boldsymbol{o}_{\mathbf{1}}$-TS5-cf8 & 35.7 & 34.6 & 37.2 \\
\hline
\end{tabular}

For cooperative MPAA/DG mechanism, using a Boltzmann distribution of all low-energy transition-state conformations, the theoretical $m: p: o_{1}$ regioselectivities were computed to be 78:11:11, 67:21:12 and 67:8:25 at the level of B3LYP-D3, M06 and $\omega \mathrm{B} 97 \mathrm{X}-\mathrm{D}$, respectively. For $\mathrm{Pd}(\mathrm{OAc})_{2}$-catalyzed $\mathrm{C}-\mathrm{H}$ activation mechanism, using a Boltzmann distribution of all low-energy transition-state conformations, the theoretical $m: p: o_{1}$ regioselectivities were computed to be 80:6:14, 39:21:40 and 57:9:34 at the level of B3LYP-D3, M06 and $\omega$ B97X-D, respectively. 
Table S2. Energies in Figure 2, Figure 3, Figure 4, Figures S2 to S14. Zero-point correction (ZPE), thermal correction to enthalpy (TCH), thermal correction to Gibbs free energy (TCG), energies (E), enthalpies (H), and Gibbs free energies (G) (in Hartree) of the structures calculated at the B3LYP-D3/6-311++G**-SDD-IEFPCM(HFIP)//B3LYP-D3/6-31G*-LANL2DZ level of theory

\begin{tabular}{|c|c|c|c|c|c|c|c|}
\hline structures & $Z P E$ & $\mathrm{TCH}$ & $T C G$ & $E$ & $\boldsymbol{H}$ & $G$ & $\begin{array}{l}\text { Imaginary } \\
\text { Frequency }\end{array}$ \\
\hline 1 & 0.309168 & 0.329264 & 0.257993 & -938.191107 & -938.190163 & -938.261433 & \\
\hline 2 & 0.416634 & 0.443568 & 0.359097 & -1282.702352 & -1282.701408 & -1282.785879 & \\
\hline 3 & 0.316228 & 0.351727 & 0.245010 & -1751.017092 & -1751.016147 & -1751.122864 & \\
\hline 4 & 0.124478 & 0.133268 & 0.091933 & -345.657077 & -345.656133 & -345.697468 & \\
\hline 5 & 0.228533 & 0.243941 & 0.184119 & -707.226347 & -707.225403 & -707.285224 & \\
\hline INT1 & 0.516977 & 0.552507 & 0.446775 & -1771.027290 & -1771.026346 & -1771.132078 & \\
\hline$m$-TS1-cf1 & 0.512456 & 0.547137 & 0.444708 & -1771.011390 & -1771.010445 & -1771.112874 & $-1382.75 i$ \\
\hline INT2 & 0.517648 & 0.552807 & 0.449147 & -1771.022463 & -1771.021519 & -1771.125179 & \\
\hline INT3 & 0.479251 & 0.513177 & 0.414996 & -1638.506615 & -1638.505671 & -1638.603852 & \\
\hline$m$-TS2 & 0.477270 & 0.511019 & 0.411016 & -1638.485623 & -1638.484679 & -1638.584682 & $-245.22 i$ \\
\hline INT4 & 0.479221 & 0.513092 & 0.412163 & -1638.528402 & -1638.527458 & -1638.628386 & \\
\hline$m$-TS3 & 0.474094 & 0.508290 & 0.404986 & -1638.498455 & -1638.497511 & -1638.600815 & $-625.21 i$ \\
\hline INT5 & 0.476674 & 0.511148 & 0.409160 & -1638.509299 & -1638.508355 & -1638.610342 & \\
\hline$p$-TS1-cf1 & 0.512620 & 0.547090 & 0.445875 & -1771.012355 & -1771.011411 & -1771.112626 & $-1428.25 i$ \\
\hline$o_{1}$-TS1-cf1 & 0.512547 & 0.547215 & 0.444487 & -1771.008711 & -1771.007767 & -1771.110495 & $-827.73 i$ \\
\hline$m$-TS1-cf2 & 0.511629 & 0.546583 & 0.443144 & -1771.011016 & -1771.010071 & -1771.113510 & $-1236.73 i$ \\
\hline$m$-TS1-cf3 & 0.512441 & 0.547207 & 0.444169 & -1771.008544 & -1771.007600 & -1771.110639 & $-1029.41 i$ \\
\hline$m$-TS1-cf4 & 0.512188 & 0.546963 & 0.443252 & -1771.010903 & -1771.009958 & -1771.113669 & $-1369.66 i$ \\
\hline$m$-TS1-cf5 & 0.512392 & 0.547076 & 0.443456 & -1771.008146 & -1771.007202 & -1771.110823 & $-1253.82 i$ \\
\hline$m$-TS1-cf6 & 0.512684 & 0.547133 & 0.445575 & -1771.008527 & -1771.007583 & -1771.109141 & $-1329.47 i$ \\
\hline$m$-TS1-cf7 & 0.512323 & 0.547130 & 0.443729 & -1771.004925 & -1771.003981 & -1771.107381 & $-968.25 i$ \\
\hline$m$-TS1-cf8 & 0.511864 & 0.546821 & 0.443034 & -1771.006162 & -1771.005218 & -1771.109005 & $-1262.59 i$ \\
\hline
\end{tabular}




\begin{tabular}{|c|c|c|c|c|c|c|c|}
\hline$p$-TS1-cf2 & 0.512618 & 0.547202 & 0.445400 & -1771.009880 & -1771.008936 & -1771.110738 & $-1434.91 i$ \\
\hline$p$-TS1-cf3 & 0.512654 & 0.547193 & 0.445259 & -1771.008817 & -1771.007873 & -1771.109807 & $-1373.91 i$ \\
\hline$p$-TS1-cf4 & 0.512729 & 0.547272 & 0.445276 & -1771.006477 & -1771.005533 & -1771.107529 & $-1352.04 i$ \\
\hline$o_{1}$-TS1-cf2 & 0.512979 & 0.547322 & 0.446457 & -1771.007039 & -1771.006095 & -1771.106960 & $-763.79 i$ \\
\hline$o_{1}$-TS1-cf3 & 0.511629 & 0.546582 & 0.444517 & -1771.007745 & -1771.006801 & -1771.108866 & $-1090.21 i$ \\
\hline$o_{1}$-TS1-cf4 & 0.512499 & 0.547101 & 0.444782 & -1771.003981 & -1771.003037 & -1771.105357 & $-1094.74 i$ \\
\hline$o_{1}$-TS1-cf5 & 0.511687 & 0.546494 & 0.443615 & -1771.004102 & -1771.003158 & -1771.106037 & $-1341.66 i$ \\
\hline$o_{1}$-TS1-cf6 & 0.511187 & 0.546065 & 0.443599 & -1771.008166 & -1771.007222 & -1771.109689 & $-1061.75 i$ \\
\hline$o_{1}$-TS1-cf7 & 0.511687 & 0.546641 & 0.442329 & -1771.002142 & -1771.001198 & -1771.105510 & $-1110.6 i$ \\
\hline$o_{1}$-TS1-cf8 & 0.512634 & 0.547152 & 0.445208 & -1770.998689 & -1770.997745 & -1771.099689 & $-990.53 i$ \\
\hline$m$-TS4-cf1 & 0.462079 & 0.500666 & 0.387955 & -1896.117074 & -1896.116129 & -1896.228841 & $-1254.56 i$ \\
\hline$m$-TS4-cf2 & 0.462938 & 0.501644 & 0.388733 & -1896.096759 & -1896.095815 & -1896.208726 & $-1316.56 i$ \\
\hline$m$-TS4-cf3 & 0.461676 & 0.500275 & 0.387976 & -1896.116343 & -1896.115399 & -1896.227697 & $-1170.03 i$ \\
\hline$m$-TS4-cf4 & 0.462679 & 0.50 & 0.390315 & -1896.114545 & -1896.113600 & -1896.224 & $-1319.71 i$ \\
\hline$m$-TS4-cf5 & 0.462 & 0.501007 & 0.389452 & -1896.114471 & -1896.113527 & -1896.225082 & $-1184.17 i$ \\
\hline$m$-TS4-cf6 & 0.463366 & 0.501422 & 0.392496 & -1896.107643 & -1896.106699 & -1896.215626 & $-1464.67 i$ \\
\hline$m$-TS4-cf7 & 0.462066 & 0.500852 & 0.387087 & -1896.121216 & -1896.120271 & -1896.234036 & $-1356.06 i$ \\
\hline$m$-TS4-cf8 & 0.462010 & 0.500634 & 0.387487 & -1896.098991 & -1896.098046 & -1896.211194 & $-1366.83 i$ \\
\hline$m$-TS4-cf9 & 0.461887 & 0.500831 & 0.386018 & -1896.110670 & -1896.109726 & -1896.224539 & $-1385.64 i$ \\
\hline$m$-TS4-cf10 & 0.463591 & 0.501350 & 0.393808 & -1896.100043 & -1896.099099 & -1896.206641 & $-1434.34 i$ \\
\hline$m$-TS4-cf11 & 0.462278 & 0.500520 & 0.390007 & -1896.109773 & -1896.108829 & -1896.219342 & $-1285.37 i$ \\
\hline$m$-TS4-cf12 & 0.462729 & 0.500927 & 0.390287 & -1896.100896 & -1896.099952 & -1896.210591 & $-1393.53 i$ \\
\hline$m$-TS4-cf13 & 0.462722 & 0.500900 & 0.390833 & -1896.118830 & -1896.117886 & -1896.227952 & $-1212.92 i$ \\
\hline$m$-TS4-cf14 & 0.462572 & 0.501265 & 0.388484 & -1896.089444 & -1896.088499 & -1896.201280 & $-1466.91 i$ \\
\hline$m$-TS4-cf15 & 0.461691 & 0.500378 & 0.387908 & -1896.111542 & -1896.110598 & -1896.223069 & $-1291.12 i$ \\
\hline$m$-TS4-cf16 & 0.461873 & 0.500558 & 0.387140 & -1896.105978 & -1896.105034 & -1896.218452 & $-1370.03 i$ \\
\hline$p$-TS4-cf1 & 0.462098 & 0.500508 & 0.388547 & -1896.119197 & -1896.118253 & -1896.230214 & $-1356.74 i$ \\
\hline$p$-TS4-cf2 & 0.462270 & 0.500932 & 0.387921 & -1896.114252 & -1896.113308 & -1896.226319 & $-1380.40 i$ \\
\hline$p$-TS4-cf3 & 0.461979 & 0.500896 & 0.387025 & -1896.100769 & -1896.099825 & -1896.213696 & $-1344.20 i$ \\
\hline
\end{tabular}




\begin{tabular}{|c|c|c|c|c|c|c|c|}
\hline$p$-TS4-cf4 & 0.462264 & 0.500810 & 0.388155 & -1896.116049 & -1896.115105 & -1896.227760 & $-1272.49 i$ \\
\hline$p$-TS4-cf5 & 0.461616 & 0.500405 & 0.386775 & -1896.106853 & -1896.105909 & -1896.219539 & $-1480.32 i$ \\
\hline$p$-TS4-cf6 & 0.462851 & 0.501127 & 0.390796 & -1896.114503 & -1896.113559 & -1896.223889 & $-1364.34 i$ \\
\hline$p$-TS4-cf7 & 0.461577 & 0.500469 & 0.386510 & -1896.103356 & -1896.102412 & -1896.216370 & $-1431.90 i$ \\
\hline$p$-TS4-cf8 & 0.462267 & 0.501034 & 0.387001 & -1896.106942 & -1896.105998 & -1896.220031 & $-1477.56 i$ \\
\hline$o_{1}$-TS4-cf1 & 0.461992 & 0.500664 & 0.388952 & -1896.123000 & -1896.122056 & -1896.233768 & $-1262.03 i$ \\
\hline$o_{1}$-TS4-cf2 & 0.462888 & 0.501437 & 0.389582 & -1896.110506 & -1896.109562 & -1896.221417 & $-1333.46 i$ \\
\hline$o_{1}$-TS4-cf3 & 0.462216 & 0.501013 & 0.388987 & -1896.108430 & -1896.107486 & -1896.219512 & $-1273.06 i$ \\
\hline$o_{1}$-TS4-cf4 & 0.462446 & 0.500629 & 0.391985 & -1896.102293 & -1896.101349 & -1896.209993 & $-1251.70 i$ \\
\hline$o_{1}$-TS4-cf5 & 0.462953 & 0.501135 & 0.391070 & -1896.113996 & -1896.113052 & -1896.223117 & $-1401.56 i$ \\
\hline$o_{1}$-TS4-cf6 & 0.462038 & 0.500944 & 0.387049 & -1896.116716 & -1896.115772 & -1896.229668 & $-1192.56 i$ \\
\hline$o_{1}$-TS4-cf7 & 0.462509 & 0.500892 & 0.390246 & -1896.111528 & -1896.110584 & -1896.221230 & $-1430.51 i$ \\
\hline$o_{1}$-TS4-cf8 & 0.462643 & 0.501005 & 0.390030 & -1896.088634 & -1896.087689 & -1896.198664 & $-1386.09 i$ \\
\hline$o_{2}$-TS1-a & 0.461310 & 0.500516 & 0.384909 & -1896.111259 & -1896.110315 & -1896.225922 & $-1305.36 i$ \\
\hline$o_{2}$-TS1-b & 0.397899 & 0.431262 & 0.329580 & -1667.055536 & -1667.054592 & -1667.156274 & $-1199.09 i$ \\
\hline$o_{2}$-TS1-c & 0.409955 & 0.441150 & 0.344899 & -1521.823286 & -1521.822342 & -1521.918593 & $-1282.23 i$ \\
\hline$o_{2}$-TS1-d & 0.512185 & 0.547440 & 0.443027 & -1770.970801 & -1770.969857 & -1771.074270 & $-1414.00 i$ \\
\hline$o_{2}$-TS1-e & 0.511850 & 0.547245 & 0.442776 & -1770.963372 & -1770.962428 & -1771.066897 & $-1369.97 i$ \\
\hline$o_{2}$-TS1-f & 0.511993 & 0.547096 & 0.443704 & -1770.971589 & -1770.970644 & -1771.074037 & $-1329.76 i$ \\
\hline$o_{2}$-TS1-g & 0.511809 & 0.547069 & 0.443035 & -1770.969362 & -1770.968418 & -1771.072452 & $-1481.38 i$ \\
\hline$o_{2}$-TS1-h & 0.564952 & 0.607455 & 0.486074 & -2145.253973 & -2145.253029 & -2145.374410 & $-1430.53 i$ \\
\hline$o_{2}$-TS1-i & 0.564281 & 0.607118 & 0.484836 & -2145.264463 & -2145.263519 & -2145.385801 & $-1407.98 i$ \\
\hline$o_{2}-\mathrm{TS} 1-\mathrm{j}$ & 0.628238 & 0.676598 & 0.540595 & -2374.309059 & -2374.308115 & -2374.444119 & $-1433.82 i$ \\
\hline$o_{2}$-TS1-k & 0.628049 & 0.676851 & 0.540371 & -2374.320992 & -2374.320048 & -2374.456528 & $-1428.12 i$ \\
\hline$m$-TS5-cf1 & 0.410138 & 0.440926 & 0.347026 & -1521.843796 & -1521.842851 & -1521.936751 & $-1092.95 i$ \\
\hline$m$-TS6 & 0.512375 & 0.547436 & 0.443253 & -1770.996241 & -1770.995296 & -1771.099479 & $-1253.60 i$ \\
\hline$m$-TS7 & 0.346358 & 0.371640 & 0.289553 & -1292.781199 & -1292.780255 & -1292.862343 & $-1249.70 i$ \\
\hline$m$-TS8 & 0.408490 & 0.440825 & 0.337701 & -1521.802869 & -1521.801925 & -1521.905049 & $-1059.97 i$ \\
\hline$m$-TS5-cf2 & 0.409727 & 0.440786 & 0.345468 & -1521.840196 & -1521.839252 & -1521.934570 & $-981.05 i$ \\
\hline
\end{tabular}




\begin{tabular}{|c|c|c|c|c|c|c|c|}
\hline$m$-TS5-cf3 & 0.409977 & 0.440845 & 0.347308 & -1521.839647 & -1521.838703 & -1521.932239 & $-1219.61 i$ \\
\hline$m$-TS5-cf4 & 0.409878 & 0.440752 & 0.346809 & -1521.839286 & -1521.838341 & -1521.932284 & $-1119.16 i$ \\
\hline$m$-TS5-cf5 & 0.409952 & 0.440874 & 0.346425 & -1521.834691 & -1521.833747 & -1521.928195 & $1031.81 i$ \\
\hline$m$-TS5-cf6 & 0.409908 & 0.440630 & 0.347090 & -1521.836620 & -1521.835676 & -1521.929215 & $-1145.62 i$ \\
\hline$m$-TS5-cf7 & 0.409927 & 0.440913 & 0.345898 & -1521.831456 & -1521.830512 & -1521.925527 & $-904.791 i$ \\
\hline$m$-TS5-cf8 & 0.409577 & 0.440410 & 0.347184 & -1521.834283 & -1521.833339 & -1521.926564 & $-1051.20 i$ \\
\hline$p$-TS5-cf1 & 0.410032 & 0.440883 & 0.347350 & -1521.841809 & -1521.840865 & -1521.934398 & $-1274.18 i$ \\
\hline$p$-TS5-cf2 & 0.410238 & 0.440859 & 0.348314 & -1521.842080 & -1521.841135 & -1521.933680 & $-1297.48 i$ \\
\hline$p$-TS5-cf3 & 0.410430 & 0.440993 & 0.348302 & -1521.836171 & -1521.835227 & -1521.927917 & $-1278.45 i$ \\
\hline$p$-TS5-cf4 & 0.410160 & 0.440852 & 0.347647 & -1521.834375 & -1521.833430 & -1521.926635 & $-1252.69 i$ \\
\hline$o_{1}$-TS5-cf1 & 0.409737 & 0.440678 & 0.346225 & -1521.840016 & -1521.839072 & -1521.933524 & $-1155.41 i$ \\
\hline$o_{1}$-TS5-cf2 & 0.409733 & 0.440762 & 0.346513 & -1521.837119 & -1521.836175 & -1521.930425 & $-956.71 i$ \\
\hline$o_{1}$-TS5-cf3 & 0.410040 & 0.440781 & 0.347018 & -1521.830652 & -1521.829708 & -1521.923471 & $-762.58 i$ \\
\hline$o_{1}$-TS5-cf4 & 0.409707 & 0.440382 & 0.347091 & -1521.835405 & -1521.834461 & -1521.927752 & $-1114.12 i$ \\
\hline$o_{1}$-TS5-cf5 & 0.409848 & 0.440646 & 0.347583 & -1521.826895 & -1521.825951 & -1521.919014 & $-1000.81 i$ \\
\hline$o_{1}$-TS5-cf6 & 0.409730 & 0.440631 & 0.346628 & -1521.828948 & -1521.828004 & -1521.922007 & $-1025.36 i$ \\
\hline$o_{1}$-TS5-cf7 & 0.409906 & 0.440861 & 0.345191 & -1521.830008 & -1521.829064 & -1521.924734 & $-964.33 i$ \\
\hline$o_{1}$-TS5-cf8 & 0.410574 & 0.441287 & 0.347602 & -1521.824553 & -1521.823609 & -1521.917295 & $-1016.73 i$ \\
\hline$m$-TS9 & 0.399061 & 0.431785 & 0.332638 & -1667.065446 & -1667.064502 & -1667.163649 & $-1112.36 i$ \\
\hline$p$-TS9 & 0.398986 & 0.431731 & 0.332480 & -1667.064756 & -1667.063812 & -1667.163063 & $-1230.78 i$ \\
\hline$o_{1}$-TS9 & 0.398665 & 0.431478 & 0.332281 & -1667.065161 & -1667.064217 & -1667.163415 & $-1292.58 i$ \\
\hline$m$-TS10 & 0.409737 & 0.440567 & 0.346818 & -1521.829693 & -1521.828749 & -1521.922498 & $-1307.63 i$ \\
\hline$p$-TS10 & 0.409778 & 0.440444 & 0.347509 & -1521.827966 & -1521.827022 & -1521.919956 & $-1345.82 i$ \\
\hline$o_{1}$-TS10 & 0.407937 & 0.439221 & 0.343401 & -1521.821831 & -1521.820886 & -1521.916707 & $-1086.09 i$ \\
\hline
\end{tabular}




\section{Cartesian Coordinates for Calculated Species}

C

C

C

C

C

C

$\mathrm{H}$

$\mathrm{H}$

$\mathrm{H}$

$\mathrm{H}$

$\mathrm{H}$

C

$\mathrm{H}$

$\mathrm{H}$

C

$\mathrm{H}$

$\mathrm{H}$

C

O

$\mathrm{N}$

C

C

C

C

C

$\mathrm{H}$

C

$\mathrm{H}$

$\mathrm{H}$

$\mathrm{H}$

C

$\mathrm{H}$

$\mathrm{H}$

$\mathrm{H}$

C

o

O

H
$-5.10612900$

$-4.85671200$

$-3.86280800$

$-3.10318200$

$-3.36471000$

$-4.35581000$

$-5.88142800$

$-5.43860300$

$-3.67522000$

$-2.78545400$

$-4.54658900$

$-1.99366000$

$-1.94695000$

$-2.17839900$

$-0.62901100$

$-0.63298000$

$-0.43214200$

0.50816900

0.32734700

1.76472700

2.02208300

2.29688700

2.05791000

2.59519800

2.34444400

1.85370600

2.61520000

2.80373400

2. 35860600

2. 84071900

2.93030700

3. 66519000

2. 59316100

3. 38864200

2.23830900

1.75215600

2.79150300

2.67395200
$-1.44214600$

$-0.82512600$

0.15033500

0.52806000

$-0.09911200$

$-1.07507700$

$-2.19908800$

$-1.09988200$

0.63042100

0.18700100

$-1.54553400$

1.54830000

2.17973500

2.22408100

0.86979100

0.24483900

0.18902500

1.88333900

3.08026000

1.37111100

$-0.02969700$

$-0.85324700$

$-0.58658500$

$-2.20998700$

$-1.93781300$

0.06576600

$-2.75228900$

$-2.83821800$

$-2.35052100$

$-3.80556200$

2. 24247200

2. 00379300

3.27323500

2.12243900

$-0.31625300$

0.74457700

$-1.16436600$

$-0.73285900$
0.21833300

$-1.00912000$

$-1.11370700$

0.00108500

1.22772200

1.33844400

0.30299300

$-1.88540200$

$-2.07203000$

2.10349200

2.29981300

$-0.11878800$

0.77440300

$-0.96072900$

$-0.30779400$

$-1.20941700$

0.52961800

$-0.36980500$

$-0.19477400$

$-0.65234500$

$-0.75959100$

0.35698700

$-2.04154200$

0.14944000

$-2.23334100$

$-2.88512200$

$-1.13278000$

1.00750100

$-3.23820900$

$-1.27091200$

$-0.51206300$

$-1.28715600$

$-0.62115000$

0.47740800

1.74585700

2.08516200

2.65281000

3. 51969300 
C

C

C

C

C

C

$\mathrm{H}$

$\mathrm{H}$

$\mathrm{H}$

$\mathrm{H}$

C

$\mathrm{H}$

$\mathrm{H}$

C

$\mathrm{H}$

$\mathrm{H}$

C

O

$\mathrm{N}$

C

C

C

C

C

$\mathrm{H}$

C

$\mathrm{H}$

$\mathrm{H}$

$\mathrm{H}$

C

$\mathrm{H}$

$\mathrm{H}$

$\mathrm{H}$

C

O

O

C

O

O

C

C

$\mathrm{H}$
$-1.84465400$

$-1.00875100$

0.36970100

0.94246600

0.09014500

$-1.29632300$

$-2.91360900$

$-1.42860500$

1.00920600

0.48349100

2. 44763400

2.79868800

2.91595500

2.94924800

2.39709200

4.00018500

2.86919900

2.65983400

3.06954200

2.89158100

1.63655400

4.02786400

1.56876100

3.94644700

4.97725200

2. 71047300

0.60369200

4.84317300

2.63328100

3.22660900

3.66080000

3.91585100

2. 26231600

0.38176800

0.34901200

$-0.72093500$

$-3.86360900$

$-3.20775800$

$-5.19237300$

$-3.33677400$

$-2.05655000$

$-4.01320600$
3.58197500

$-0.67109900$

$4.64481200-1.00239100$

$4.56028600-0.78122400$

$3.41352900-0.21274100$

$2.36762200 \quad 0.13703800$

$2.42244000-0.09619400$

$3.65279300-0.85113000$

$5.54654500-1.44028000$

$5.39630300-1.05750300$

$1.46283400 \quad 0.58218700$

$3.32914100-0.02422500$

$4.25690000 \quad 0.44575500$

$3.27718700-1.01416200$

$2.13883000 \quad 0.80295200$

$2.06560500 \quad 1.74694400$

$2.30702800 \quad 1.07597600$

$0.81810700 \quad 0.02742400$

$0.79328400-1.17961800$

$\begin{array}{ll}-0.33812400 & 0.74894600\end{array}$

$-1.58676900$

$-2.21985800$

$-2.18613100$

$-3.44668400$

$-3.40896000$

$-1.66911700$

$-4.04291500$

$-3.92792900$

$-3.85985700$

$-4.99530300$

$-0.38330000$

$-1.35008000$

0.39510600

$-0.27817500$

$-1.62477100$

$-0.72729400$

$-2.18803900$

$-0.39367600$

$-1.33771700$

0.06227400

$-0.07098400$

$-0.48361100$

$-0.75085200$

$-1.14813400$

$-0.38446800$

$-1.27953900$

$-0.85689200$

$-1.56417500$

$-1.79611200$

2. 20287400

2.46920800

2.54063500

2.71037200

0.48587900

1.31793100

$-0.01733800$

0.32526000

0.76952600

$-0.47503200$

0.12367900

0.92181900

$-0.05123100$

1.22607000

0.24372300

1.59318900

$-0.56995200$ 
$-1.48757300$

$-5.82410600$

$-5.42945900$

$-6.87988800$

$-5.61326200$

$-4.55218100$

$-6.16543400$

$-5.97232400$

$-1.53424300$
0.47516100 $-1.74473800$ $-2.12870900$ $-1.49251600$ $-2.74030100$ $-2.98414300$ $-3.66392000$ $-2.32723700$ $-1.78558800$
0.78618400

0.41747500

1.36214400

0.54200200

$-0.71446500$

$-0.81166400$

$-0.50650700$

$-1.66273900$

0.38885100
$-1.60729700$

0.05147900

$-1.02399400$

1.14953900

2.28655900

1. 60988000

2.31569900

3.19214300

3. 46560200

4.10422600

2.70181700

0.00914000

$-0.74016700$

0.98632100

$-0.30867500$

0.00068700

2.22879900

2. 65275500

1.16178600

$-0.05354400$

$-1.15117500$

1.02210100

$-0.01229000$

0.74276700

$-0.98782400$

0.29692000

3.10079400

3.08480800

2. 74339200

4.13379800

1.60667700

$-2.22793000$
0.92650500

2. 58515400

2.45679400

1. 98243700

$-1.24385400$

$-2.11098400$

0.00791900

$-1.77058100$

$-0.96877900$

$-2.16126700$

$-2.59030900$

3. 57557300

3.24639000

3. 66050900

4.55024800

$-1.85216200$

$-1.34488100$

$-0.33899400$

$-1.99793700$

2. 58470200

1. 98125000

2. 45721900

3. 57558000

3.25196400

3. 65438700

4.55226300

$-1.80561900$

$-1.03833700$

$-2.75406200$

$-1.90301500$

0.92781500

$-1.34670000$
$-0.00577900$

1.88200200

1.22620500

1.68907200

$-1.86842500$

$-1.24185200$

$-1.66889200$

$-2.96269600$

$-3.65073400$

$-2.49686500$

$-3.49239100$

3.02734600

3. 75416600

3.50435200

2. 64427500

$-0.00002500$

1.87185100

1.22877700

1.66968800

$-1.88241400$

$-1.68927900$

$-1.22673900$

$-3.02741000$

$-3.75070300$

$-3.50881500$

$-2.64229100$

3. 02157100

3. 80283200

3.42459500

2. 67541700

0.00575300

$-1.87172400$ 


$\begin{array}{lrrr}\mathrm{O} & -2.65262300 & -0.34118500 & -1.22856100 \\ \mathrm{O} & -1.16028000 & -1.99879000 & -1.66978200 \\ \mathrm{C} & -3.09974200 & -1.80821300 & -3.02127000 \\ \mathrm{H} & -3.08417800 & -1.04113900 & -3.80274700 \\ \mathrm{H} & -2.74184400 & -2.75656600 & -3.42406800 \\ \mathrm{H} & -4.13268100 & -1.90605500 & -2.67505600 \\ \mathrm{C} & -2.28532700 & -1.24551600 & 1.86860900 \\ \mathrm{O} & -2.31516100 & 0.00627100 & 1.66918400 \\ \mathrm{O} & -1.60832900 & -2.11222600 & 1.24180200 \\ \mathrm{C} & -3.19048500 & -1.77275500 & 2.96297900 \\ \mathrm{H} & -4.10315100 & -2.16236300 & 2.49737700 \\ \mathrm{H} & -2.70034500 & -2.59326800 & 3.49161400 \\ \mathrm{H} & -3.46303200 & -0.97140400 & 3.65191100\end{array}$

C

$\mathrm{H}$

$\mathrm{H}$

C

$\mathrm{H}$

C

O

O

C

$\mathrm{H}$

$\mathrm{H}$

C

$\mathrm{H}$

$\mathrm{H}$

$\mathrm{H}$
$-2.99560400$

$-3.21541100$

$-3.83177900$

$-1.72992700$

$-1.46670600$

$-0.60681300$

$-0.71748000$

0.58224100

1.75322900

1.72196100

1.72208300

2.96676600

3. 88400400

2.97098600

2. 97087300
$-0.33507600$

0.72913500

$-1.02788200$

$-0.75941900$

$-1.81315200$

0.21374900

1.42441000

$-0.43162700$

0.41404100

1.06207100

1.06196700

$-0.49681900$

0.10221800

$-1.13779600$

$-1.13768400$
$-0.00006500$

$-0.00005000$

$-0.00012900$

$-0.00000100$

$-0.00001100$

0.00010100

0.00001500

0.00006800

0.00003300

$-0.88273000$

0.88287700

$-0.00010200$

$-0.00012300$

0.88747100

$-0.88775600$

\section{5}

C

$\mathrm{H}$

$\mathrm{H}$

$\mathrm{H}$

C

O

$\mathrm{N}$

C

$\mathrm{H}$
$-2.94405600$

$-2.11307900$

$-3.08843500$

$-3.85971000$

$-2.69568400$

$-3.59846800$

$-1.37519500$

$-0.99000800$

$-1.82803400$
$-2.19703500$

$-2.91047900$

$-1.86726400$

$-2.68937000$

$-0.98023300$

$-0.37296400$

$-0.61268900$

0.64102400

0.94019800
$-0.87271800$

$-0.85313500$

$-1.90869900$

$-0.54019200$

0.00429600

0.56361200

0.11616400

0.72419600

1. 36301700 


$\begin{array}{lrrr}\mathrm{C} & -0.80997200 & 1.78364300 & -0.28058800 \\ \mathrm{O} & -0.35804000 & 2.87006700 & 0.01114600 \\ \mathrm{O} & -1.21298600 & 1.46534500 & -1.52971600 \\ \mathrm{H} & -1.07516400 & 2.26724000 & -2.06932000 \\ \mathrm{H} & -0.69456600 & -1.05509700 & -0.48699700 \\ \mathrm{C} & 0.28322300 & 0.49822600 & 1.59417900 \\ \mathrm{H} & 0.50375700 & 1.48101600 & 2.02048400 \\ \mathrm{H} & 0.04387800 & -0.18262500 & 2.41737200 \\ \mathrm{C} & 1.46917900 & -0.02081600 & 0.80893800 \\ \mathrm{C} & 2.30975000 & 0.85997300 & 0.11108400 \\ \mathrm{C} & 1.71594600 & -1.39880300 & 0.71614500 \\ \mathrm{C} & 3.36816900 & 0.37383400 & -0.65781600 \\ \mathrm{H} & 2.12411700 & 1.92864800 & 0.17182200 \\ \mathrm{C} & 2.77363600 & -1.88702800 & -0.05451100 \\ \mathrm{H} & 1.07576400 & -2.09076500 & 1.25889200 \\ \mathrm{C} & 3.60295700 & -1.00068200 & -0.74456700 \\ \mathrm{H} & 4.01295100 & 1.07032400 & -1.18743400 \\ \mathrm{H} & 2.95271500 & -2.95767000 & -0.10989400 \\ \mathrm{H} & 4.42928000 & -1.37753400 & -1.34121500\end{array}$

\section{INT1}

C
C
C
C
C
H
H
H
H
H
C
H
H
C
H
H
C
O
N
C
C

$$
\begin{array}{r}
0.00402300 \\
0.84207500 \\
2.23574600 \\
2.80610900 \\
1.96117000 \\
0.58659300 \\
-1.04061500 \\
0.38573800 \\
2.87971800 \\
2.40862900 \\
-0.05135100 \\
4.30703300 \\
4.80263500 \\
4.59787000 \\
4.81435900 \\
5.88888900 \\
4.31248900 \\
4.61018300 \\
4.73748600 \\
4.27491700 \\
4.19062000 \\
2.95085600
\end{array}
$$

$-2.43124900$

1.35588900

$-2.97046700$

$-2.82501100$

0.35162600

0.45080000

$-2.16940800$

1. 53935000

$-1.60696000$

$-1.73603900$

2.52413300

2. 44566000

$-2.71925600$

$-3.56416100$

1.38368600

$-0.43192200$

$-3.23597800$

$-0.31891600$

$-1.08728500$

$-1.33468600$

3. 36867800

3.22786700

$-2.03144700$

1.66079900

$-2.87286600$

$-2.03915100$

1.16917000

2.71787800

$-0.72537900$

1.02169600

$-0.61231700$

1.21728500

0.13298200

1.47450700

$-0.75863000$

$-1.80195300$

$-0.49313300$

$-1.12559500$

0.42876600

$-1.10423300$

1. 66587900

$-0.39851100$

2.23515900

$-0.05156600$ 


\begin{tabular}{|c|c|c|c|}
\hline C & 5.36844300 & 2.36218000 & -0.11098600 \\
\hline C & 2.91697900 & 3.49571200 & 0.56227000 \\
\hline C & 5.32768500 & 3.59705600 & 0.53616200 \\
\hline $\mathrm{H}$ & 6.31439300 & 1.91949000 & -0.40797500 \\
\hline C & 4.09791600 & 4.16709500 & 0.87102700 \\
\hline $\mathrm{H}$ & 1.95516000 & 3.93648700 & 0.80143800 \\
\hline $\mathrm{H}$ & 6.25316300 & 4.11852100 & 0.76299900 \\
\hline $\mathrm{H}$ & 4.05691900 & 5.13457400 & 1.36224200 \\
\hline C & 3.93252200 & 0.44228900 & -2.52717600 \\
\hline $\mathrm{H}$ & 2.86110700 & 0.62903700 & -2.66693000 \\
\hline $\mathrm{H}$ & 4.50233700 & 1.22070300 & -3.04452700 \\
\hline $\mathrm{H}$ & 4.18011500 & -0.53595500 & -2.93808800 \\
\hline C & 1.65592700 & 1.54360600 & -0.31843900 \\
\hline O & 1.52565500 & 0.31471100 & -0.14660000 \\
\hline O & 0.71635900 & 2.36047500 & -0.71462900 \\
\hline $\mathrm{H}$ & -0.14598900 & 1.85998200 & -0.96855200 \\
\hline $\mathrm{Pd}$ & -0.37307200 & -0.78297700 & -0.31697200 \\
\hline C & -3.19070300 & -0.33628800 & -0.64171900 \\
\hline $\mathrm{N}$ & -2.24408600 & -1.44597100 & -0.57721100 \\
\hline C & -2.57311700 & -2.73410200 & -0.85305600 \\
\hline O & -1.76210800 & -3.66523200 & -0.84072200 \\
\hline C & -4.04252000 & -3.02990800 & -1.18006900 \\
\hline $\mathrm{H}$ & -4.31729900 & -2.61364300 & -2.15645500 \\
\hline $\mathrm{H}$ & -4.15226400 & -4.11446300 & -1.21698600 \\
\hline $\mathrm{H}$ & -4.73307000 & -2.62004900 & -0.43530800 \\
\hline C & -2.50358200 & 0.90985900 & -1.21317300 \\
\hline O & -3.09262600 & 1.91956100 & -1.53068000 \\
\hline O & -1.17087200 & 0.80733500 & -1.29591900 \\
\hline $\mathrm{H}$ & -4.02395300 & -0.56437100 & -1.31229600 \\
\hline $\mathrm{C}$ & -3.77337600 & -0.01676300 & 0.77133900 \\
\hline $\mathrm{H}$ & -2.97139000 & 0.41546700 & 1.38335100 \\
\hline $\mathrm{H}$ & -4.02256600 & -0.98351500 & 1.22393600 \\
\hline C & -4.99140700 & 0.87568000 & 0.75842100 \\
\hline $\mathrm{C}$ & -4.88706300 & 2.26039600 & 0.93695900 \\
\hline C & -6.26093000 & 0.32155900 & 0.54402300 \\
\hline C & -6.02176800 & 3.07109900 & 0.90379500 \\
\hline $\mathrm{H}$ & -3.90802300 & 2.70631200 & 1.08733200 \\
\hline C & -7.39869200 & 1.12820700 & 0.50663400 \\
\hline $\mathrm{H}$ & -6.35838700 & -0.75452500 & 0.40960300 \\
\hline C & -7.28145900 & 2.50803900 & 0.68833900 \\
\hline $\mathrm{H}$ & -5.92117600 & 4.14439800 & 1.04185400 \\
\hline $\mathrm{H}$ & -8.37495900 & 0.67983700 & 0.34116200 \\
\hline $\mathrm{H}$ & -8.16550300 & 3.13961800 & 0.6623670 \\
\hline
\end{tabular}




\section{m-TS1-cf1}

$\begin{array}{crrr}\mathrm{C} & 0.33822900 & -2.36387700 & 1.92957900 \\ \mathrm{C} & 0.61115400 & -2.21227000 & 0.55066600 \\ \mathrm{C} & 1.94162400 & -2.36312100 & 0.10961300 \\ \mathrm{C} & 2.99395300 & -2.57830000 & 1.00774100 \\ \mathrm{C} & 2.68360400 & -2.71028000 & 2.36930500 \\ \mathrm{C} & 1.36613000 & -2.62768800 & 2.83055000 \\ \mathrm{H} & -0.68688300 & -2.27907300 & 2.28288500 \\ \mathrm{H} & -0.36988000 & -2.78563600 & -0.20712800 \\ \mathrm{H} & 2.16773300 & -2.26272500 & -0.94740500 \\ \mathrm{H} & 3.48907200 & -2.88074600 & 3.08134600 \\ \mathrm{H} & 1.15420600 & -2.75223800 & 3.88951900 \\ \mathrm{C} & 4.43703700 & -2.56755100 & 0.54383300 \\ \mathrm{H} & 4.56121200 & -3.18141300 & -0.35346900 \\ \mathrm{H} & 5.07918200 & -2.98739600 & 1.32628800 \\ \mathrm{C} & 4.93057700 & -1.13873200 & 0.21699200 \\ \mathrm{H} & 6.02625200 & -1.14134500 & 0.13534000 \\ \mathrm{H} & 4.66931000 & -0.45105700 & 1.02600600 \\ \mathrm{C} & 4.37801600 & -0.66421800 & -1.12674200 \\ \mathrm{O} & 4.20235400 & -1.45032500 & -2.05317300 \\ \mathrm{~N} & 4.06774200 & 0.66877100 & -1.29294900 \\ \mathrm{C} & 4.19500000 & 1.67360700 & -0.29162100 \\ \mathrm{C} & 3.05666900 & 2.31704000 & 0.23980700 \\ \mathrm{C} & 5.46378500 & 2.11188300 & 0.09653600 \\ \mathrm{C} & 3.20865800 & 3.40838300 & 1.10339300 \\ \mathrm{C} & 5.60693400 & 3.16426200 & 1.00230800 \\ \mathrm{H} & 6.33413600 & 1.63081500 & -0.33882400 \\ \mathrm{C} & 4.47999500 & 3.82485400 & 1.49545500 \\ \mathrm{H} & 2.32162300 & 3.90674800 & 1.48080400 \\ \mathrm{H} & 6.60135600 & 3.48633400 & 1.29794800 \\ \mathrm{H} & 4.58943400 & 4.66042900 & 2.18003100 \\ \mathrm{C} & 3.39736500 & 1.01765800 & -2.55429800 \\ \mathrm{H} & 2.41823500 & 0.53171300 & -2.61678300 \\ \mathrm{H} & 3.27759300 & 2.10135800 & -2.60050600 \\ \mathrm{H} & 4.00298300 & 0.67716100 & -3.39691100 \\ \mathrm{C} & 1.69148000 & 1.79147400 & -0.03052800 \\ \mathrm{O} & 1.51232100 & 0.56064500 & -0.03801000 \\ \mathrm{C} & -2.76400400 & 2.69762500 & -0.20996200 \\ \mathrm{O} & -0.13899000 & 2.27975200 & -0.43484900 \\ \mathrm{~N} & -3.19534600 & -0.22749100 & -0.65471700 \\ \mathrm{C} & & & \\ & -2.14145300 & -1.24410500 & -0.58785700 \\ & & -2.52657100 & -0.83279500 \\ \mathrm{H} & & & \\ \mathrm{H} & & & \end{array}$




$\begin{array}{lrrr}\mathrm{O} & -1.38188100 & -3.38323600 & -0.72082700 \\ \mathrm{C} & -3.67430700 & -3.05374500 & -1.28361400 \\ \mathrm{H} & -3.90398900 & -2.68312000 & -2.28909800 \\ \mathrm{H} & -3.63798400 & -4.14301500 & -1.30500300 \\ \mathrm{H} & -4.47823800 & -2.72695500 & -0.61757200 \\ \mathrm{C} & -2.59436500 & 1.15361800 & -1.01016600 \\ \mathrm{O} & -3.29722800 & 2.06789000 & -1.38924000 \\ \mathrm{O} & -1.28999400 & 1.26153700 & -0.79757000 \\ \mathrm{H} & -3.92880600 & -0.47076000 & -1.42925100 \\ \mathrm{C} & -3.92304500 & -0.12338200 & 0.72356100 \\ \mathrm{H} & -3.23828600 & 0.36001100 & 1.43114800 \\ \mathrm{H} & -4.06984900 & -1.14746000 & 1.08626600 \\ \mathrm{C} & -5.25217200 & 0.59290500 & 0.67405100 \\ \mathrm{C} & -5.34537700 & 1.97558800 & 0.87481000 \\ \mathrm{C} & -6.42471200 & -0.12712000 & 0.40889400 \\ \mathrm{C} & -6.58036600 & 2.62116600 & 0.81430300 \\ \mathrm{H} & -4.44280100 & 2.54953600 & 1.06053500 \\ \mathrm{C} & -7.66223400 & 0.51453100 & 0.34439100 \\ \mathrm{H} & -6.36916100 & -1.20435300 & 0.25937200 \\ \mathrm{C} & -7.74268400 & 1.89341900 & 0.54912600 \\ \mathrm{H} & -6.63406100 & 3.69524600 & 0.97053500 \\ \mathrm{H} & -8.56121900 & -0.06161700 & 0.14107100 \\ \mathrm{H} & -8.70450200 & 2.39727900 & 0.50317400 \\ & & & \\ & & & \end{array}$

\section{INT2}

C

C

C

C

C

C

$\mathrm{H}$

$\mathrm{H}$

$\mathrm{H}$

$\mathrm{H}$

$\mathrm{H}$

C

$\mathrm{H}$

$\mathrm{H}$

C

$\mathrm{H}$

$\mathrm{H}$

C
0.13229400

0.66050000

2.02653800

2.90149600

2.35788400

0.98203800

$-0.92763400$

$-0.65654700$

2.43524300

3.01784600

0.57441900

4.40153900

4.70915300

4.91691900

4.88540900

5.96725500

4.40353300

4.66153900
$-2.39680200$

$-1.91977100$

$-2.08781000$

$-2.58389700$

$-3.03969700$

$-2.97821400$

$-2.29097600$

$-3.09870500$

$-1.75438800$

$-3.43231500$

$-3.34543800$

$-2.50623300$

$-3.10894900$

$-2.90416400$

$-1.05375000$

$-0.99898000$

$-0.36790500$

$-0.63003000$
1.69432400

0.48364100

0.22471600

1.20079100

2.40923400

2.64094800

1.91360700

$-0.62921400$

$-0.72138600$

3.17998200

3.58002600

0.98501500

0.12368700

1. 86623700

0.74121500

0.92425100

1. 44319200

$-0.71200000$ 


\begin{tabular}{|c|c|c|c|}
\hline O & 4.78739400 & -1.43732000 & -1.62774000 \\
\hline $\mathrm{N}$ & 4.29157800 & 0.67060300 & -1.00003100 \\
\hline $\mathrm{C}$ & 4.12500800 & 1.71875600 & -0.05413300 \\
\hline C & 2.88216800 & 2.37532300 & 0.11967700 \\
\hline C & 5.24083800 & 2.19238200 & 0.64325200 \\
\hline C & 2.80537600 & 3.50935400 & 0.94151800 \\
\hline C & 5.13997600 & 3.28963400 & 1.49874000 \\
\hline $\mathrm{H}$ & 6.19603000 & 1.70253200 & 0.48215300 \\
\hline C & 3.92456400 & 3.96267300 & 1.63579200 \\
\hline $\mathrm{H}$ & 1.84925800 & 4.00963300 & 1.05034900 \\
\hline $\mathrm{H}$ & 6.02023400 & 3.63588100 & 2.03297500 \\
\hline $\mathrm{H}$ & 3.84701500 & 4.83240200 & 2.28096200 \\
\hline C & 3.95038300 & 0.93569800 & -2.40545100 \\
\hline $\mathrm{H}$ & 3.05197600 & 0.38262900 & -2.69458800 \\
\hline $\mathrm{H}$ & 3.78104100 & 2.00664000 & -2.52960900 \\
\hline $\mathrm{H}$ & 4.77527300 & 0.61672700 & -3.04605900 \\
\hline $\mathrm{C}$ & 1.61787400 & 1.80989600 & -0.42071500 \\
\hline O & 1.52954100 & 0.58106900 & -0.60086300 \\
\hline O & 0.64213000 & 2.66269400 & -0.61531400 \\
\hline $\mathrm{H}$ & -0.20238100 & 2.19996800 & -0.96065800 \\
\hline $\mathrm{Pd}$ & -0.29533100 & -0.51287400 & -0.59605200 \\
\hline C & -3.18147400 & -0.24922500 & -0.72904300 \\
\hline $\mathrm{N}$ & -2.15717000 & -1.31139300 & -0.72549900 \\
\hline C & -2.45499300 & -2.55496000 & -0.90834800 \\
\hline O & -1.52717200 & -3.50262700 & -0.87991000 \\
\hline C & -3.83922200 & -3.08030200 & -1.17919500 \\
\hline $\mathrm{H}$ & -4.18626800 & -2.74432000 & -2.16264300 \\
\hline $\mathrm{H}$ & -3.82360500 & -4.17054500 & -1.16191600 \\
\hline $\mathrm{H}$ & -4.54984200 & -2.71343800 & -0.43300600 \\
\hline C & -2.62725600 & 1.07840200 & -1.32357400 \\
\hline 0 & -3.41239200 & 1.96372500 & -1.61123100 \\
\hline O & -1.31952600 & 1.15718100 & -1.42231300 \\
\hline $\mathrm{H}$ & -4.03945800 & -0.53942700 & -1.34152800 \\
\hline C & -3.66259100 & -0.00984000 & 0.73646300 \\
\hline $\mathrm{H}$ & -2.88251900 & 0.54942900 & 1.26691100 \\
\hline $\mathrm{H}$ & -3.72083800 & -0.99269900 & 1.22013800 \\
\hline C & -5.00266800 & 0.68069800 & 0.84558400 \\
\hline C & -5.10738400 & 2.07665100 & 0.87997700 \\
\hline C & -6.17625000 & -0.08253800 & 0.90390600 \\
\hline C & -6.35567100 & 2.69218900 & 0.97046200 \\
\hline $\mathrm{H}$ & -4.20875400 & 2.68063600 & 0.81108800 \\
\hline C & -7.42774500 & 0.52901100 & 0.99128200 \\
\hline $\mathrm{H}$ & -6.10948600 & -1.16963600 & 0.89160700 \\
\hline C & -7.51953500 & 1.92164800 & 1.02603200 \\
\hline
\end{tabular}


$\mathrm{H}$

$\mathrm{H}$

$\mathrm{H}$
$-6.41937500$

$-8.32662000$

$-8.49111100$
3.77675600

$-0.08024700$

2.40335100
0.99273200

1.03893000

1.09720400

\section{INT3}

C

C

C

C

C

C

$\mathrm{H}$

$\mathrm{H}$

$\mathrm{H}$

$\mathrm{H}$

C

$\mathrm{H}$

$\mathrm{H}$

C

$\mathrm{H}$

$\mathrm{H}$

C

$\mathrm{O}$

$\mathrm{N}$

C

C

C

C

C

$\mathrm{H}$

C

$\mathrm{H}$

$\mathrm{H}$

$\mathrm{H}$

C

$\mathrm{H}$

$\mathrm{H}$

$\mathrm{H}$

C

O

$O$

$\mathrm{Pd}$

C
0.22915900

$-0.01978600$

$-1.25280400$

$-2.29677100$

$-2.04351600$

$-0.78976400$

1.17314000

$-1.45453200$

$-2.84446000$

$-0.60917700$

$-3.70168900$

$-3.72102600$

$-4.34638400$

$-4.28845000$

$-5.36871800$

$-4.14338200$

$-3.69374800$

$-3.65376700$

$-3.19032000$

$-3.18866100$

$-2.00322300$

$-4.42606100$

$-2.11297600$

$-4.51419700$

$-5.32119900$

$-3.34834400$

$-1.19512800$

$-5.48742700$

$-3.40075200$

$-2.62645600$

$-1.83779500$

$-2.21100700$

$-3.41299500$

$-0.61509100$

$-0.50979100$

0.35513100

1.17122300

3.81963700
$-1.91557200$

$-1.81759000$

$-2.18108400$

$-2.58589400$

$-2.70384100$

$-2.38786700$

$-1.59128700$

$-2.06107900$

$-3.01667100$

$-2.47172800$

$-2.70104500$

$-3.26805600$

$-3.21763000$

$-1.29433000$

$-1.38271600$

$-0.65148900$

$-0.70377700$

$-1.38310000$

0.57518000

1.46364000

1. 91239200

1.95059600

2.84311300

2.85375400

1. 60997900

3.31026000

3.17669300

3. 21162000

4.02506500

1.03992700

0.36467500

2.03698400

1.07306200

1. 41372200

0.52052900

1.87763800

$-0.72919300$

$-0.83215100$
$-1.83332000$

$-0.46236500$

0.07278300

$-0.77344100$

$-2.14615300$

$-2.66897500$

$-2.26034700$

1.13243500

$-2.81243600$

$-3.73807800$

$-0.22231400$

0.71387400

$-0.94239900$

0.05787200

0.23198900

$-0.81283700$

1.33868200

2. 36385200

1. 34184400

0.22495500

$-0.40800400$

$-0.21795400$

$-1.45357600$

$-1.27477900$

0.29376700

$-1.89166300$

$-1.92412700$

$-1.59958000$

$-2.70787300$

2. 61565800

2.94896700

2.46895200

3.37658200

$-0.09033700$

0.81822400

$-0.74569000$

0.71143800

$-0.44136500$ 
4.67832000

3.49263200

2. 91124200

2.70726500

2.53444900

2.18355600

3.23569300

4.03225400

4.24393200

4.97119800

2.98068400

2.05542000

3. 34288100

2. 76232200

2.95277800

2. 60378000

4.17058000

4.22537000

4.17202000

5.04167800

2.38978200

1. 72721100
$-0.30338900$

$-0.48266200$

$-1.93223600$

$-2.22714700$

$-2.59162400$

$-3.13345000$

$-1.67521800$

0.76145100

1. 42862200

0.52988000

1.33864100

1.53469200

2.28026800

0.64738900

1.70130900

2. 33806600

2. 26720500

3. 35066400

1. 99181800

1.80923700

0.68988400

2.01033800
0.23561300

$-1.69772100$

0.01328100

1.34433400

$-0.76056400$

1. 63242100

2. 11664500

$-2.22112300$

$-1.38318400$

$-2.73538900$

$-3.15106500$

$-2.60002000$

$-3.57852500$

$-3.97188000$

1. 52196100

0.42976000

2.19814800

2. 07357200

3.25397300

1.71556100

1.96557700

0.00511200

\section{m-TS2}

C

C

C

C

C

C

$\mathrm{H}$

$\mathrm{H}$

$\mathrm{H}$

$\mathrm{H}$

C

$\mathrm{H}$

$\mathrm{H}$

C

$\mathrm{H}$

$\mathrm{H}$

C

O

N
$-0.38433200$

$-0.05579700$

$-0.97651400$

$-2.27640800$

$-2.60543100$

$-1.66800400$

0.34421200

$-0.72515000$

$-3.61102000$

$-1.93945100$

$-3.33869500$

$-2.91612700$

$-4.12638400$

$-3.96967600$

$-4.84397600$

$-4.31650700$

$-2.98774600$

$-2.39810700$

$-2.77941900$
$-1.71476700$

$-1.80445700$

$-2.29371000$

$-2.62286000$

$-2.51265700$

$-2.07048000$

$-1.35701100$

$-2.34238000$

$-2.76990500$

$-1.99369000$

$-2.95007100$

$-3.51268600$

$-3.56138500$

$-1.65482600$

$-1.91398500$

$-1.02357900$

$-0.93710300$

$-1.57119100$

0.41048300
$-2.40704700$

$-1.04747800$

$-0.11724000$

$-0.52587000$

$-1.88413000$

$-2.81987400$

$-3.12934700$

0.93833400

$-2.20943300$

$-3.86946900$

0.49963300

1.33742800

0.04414700

1.05888400

1.67011800

0.23804400

1.98755500

2. 86183200

1.84305500 


\begin{tabular}{|c|c|c|c|}
\hline $\mathrm{C}$ & -3.36366000 & 1.21692200 & 0.81722500 \\
\hline $\mathrm{C}$ & -2.62659000 & 1.72476600 & -0.27874400 \\
\hline $\mathrm{C}$ & -4.72146400 & 1.53961500 & 0.94039200 \\
\hline $\mathrm{C}$ & -3.28770300 & 2.54136900 & -1.20934200 \\
\hline $\mathrm{C}$ & -5.36500400 & 2.33382500 & -0.00661700 \\
\hline $\mathrm{H}$ & -5.26259600 & 1.15268300 & 1.79879700 \\
\hline $\mathrm{C}$ & -4.64143000 & 2.84175300 & -1.08672800 \\
\hline $\mathrm{H}$ & -2.70603800 & 2.92461200 & -2.04009400 \\
\hline $\mathrm{H}$ & -6.42059800 & 2.56474300 & 0.10857200 \\
\hline $\mathrm{H}$ & -5.12745400 & 3.47066000 & -1.82731900 \\
\hline $\mathrm{C}$ & -1.82366700 & 1.02333000 & 2.77360400 \\
\hline $\mathrm{H}$ & -0.87283100 & 0.49091500 & 2.74187700 \\
\hline $\mathrm{H}$ & -1.66893600 & 2.06148900 & 2.47849800 \\
\hline $\mathrm{H}$ & -2.22376200 & 0.98371800 & 3.79233400 \\
\hline $\mathrm{C}$ & -1.16843200 & 1.42698100 & -0.54147100 \\
\hline 0 & -0.57488500 & 0.64704500 & 0.26819000 \\
\hline O & -0.62565900 & 1.96364200 & -1.54703200 \\
\hline $\mathrm{Pd}$ & 1.20959500 & -0.32149600 & -0.35870100 \\
\hline C & 3.71658400 & -1.31397100 & 0.43619400 \\
\hline O & 3.47093600 & -1.78860300 & 1.52871900 \\
\hline O & 4.75665900 & -0.49855700 & 0.17687200 \\
\hline C & 2.90154500 & -1.52774800 & -0.79394200 \\
\hline $\mathrm{C}$ & 1.92856600 & -2.55697700 & -0.80429400 \\
\hline $\mathrm{H}$ & 3.34889200 & -1.19832000 & -1.72925100 \\
\hline $\mathrm{H}$ & 1.74237100 & -3.09597300 & -1.72345900 \\
\hline $\mathrm{H}$ & 1.77432700 & -3.11724900 & 0.11352800 \\
\hline $\mathrm{C}$ & 5.51104400 & -0.06628800 & 1.33085900 \\
\hline $\mathrm{H}$ & 4.82680600 & 0.45369000 & 2.00947000 \\
\hline $\mathrm{H}$ & 5.90108300 & -0.94500900 & 1.85457900 \\
\hline $\mathrm{C}$ & 6.61928100 & 0.83911500 & 0.82751900 \\
\hline $\mathrm{H}$ & 6.20283800 & 1.70352700 & 0.30040500 \\
\hline $\mathrm{H}$ & 7.21875300 & 1.20130600 & 1.66982000 \\
\hline $\mathrm{H}$ & 7.27857600 & 0.30105600 & 0.13874200 \\
\hline $\mathrm{C}$ & 2.50495100 & 2.46300800 & -0.30483500 \\
\hline 0 & 1.67977500 & 2.92092400 & -1.19796900 \\
\hline C & 3.63361000 & 3.40621400 & 0.03302200 \\
\hline $\mathrm{H}$ & 4.35009300 & 3.40768600 & -0.79670900 \\
\hline $\mathrm{H}$ & 3.25554700 & 4.42531000 & 0.14725500 \\
\hline $\mathrm{H}$ & 4.13894600 & 3.07663400 & 0.94152900 \\
\hline 0 & 2.44900300 & 1.34843700 & 0.24480800 \\
\hline $\mathrm{H}$ & 0.80101100 & 2.36912400 & -1.34653600 \\
\hline
\end{tabular}

\section{INT4}




\begin{tabular}{|c|c|c|c|}
\hline C & 0.55659000 & -2.41374600 & -2.17115500 \\
\hline $\mathrm{C}$ & 0.93629300 & -2.60296900 & -0.81394300 \\
\hline $\mathrm{C}$ & -0.08006000 & -2.84806300 & 0.14562700 \\
\hline $\mathrm{C}$ & -1.42287400 & -2.93520300 & -0.22809000 \\
\hline C & -1.75315600 & -2.78372000 & -1.58406800 \\
\hline C & -0.78292300 & -2.51829600 & -2.54779000 \\
\hline $\mathrm{H}$ & 1.32723300 & -2.25029400 & -2.91985400 \\
\hline $\mathrm{H}$ & 0.19186700 & -2.94621600 & 1.19189500 \\
\hline $\mathrm{H}$ & -2.79685500 & -2.85439300 & -1.88277600 \\
\hline $\mathrm{H}$ & -1.06529300 & -2.38910300 & -3.58835800 \\
\hline $\mathrm{C}$ & -2.51892100 & -3.10187700 & 0.80104600 \\
\hline $\mathrm{H}$ & -2.11169500 & -3.52063600 & 1.72484600 \\
\hline $\mathrm{H}$ & -3.27827900 & -3.79672000 & 0.42153000 \\
\hline $\mathrm{C}$ & -3.20063000 & -1.75882100 & 1.12276500 \\
\hline $\mathrm{H}$ & -4.09040600 & -1.93253400 & 1.74330500 \\
\hline $\mathrm{H}$ & -3.54666300 & -1.28025300 & 0.20323700 \\
\hline $\mathrm{C}$ & -2.26257100 & -0.83087800 & 1.89453400 \\
\hline O & -1.32513300 & -1.27696500 & 2.54916000 \\
\hline $\mathrm{N}$ & -2.56060200 & 0.51412800 & 1.87827800 \\
\hline $\mathrm{C}$ & -3.46286300 & 1.08930300 & 0.92069100 \\
\hline $\mathrm{C}$ & -3.04653100 & 1.46496800 & -0.37524600 \\
\hline $\mathrm{C}$ & -4.77563400 & 1.34766500 & 1.32465600 \\
\hline $\mathrm{C}$ & -3.95880800 & 2.10196500 & -1.22486300 \\
\hline $\mathrm{C}$ & -5.68397500 & 1.95510600 & 0.45648800 \\
\hline $\mathrm{H}$ & -5.06858900 & 1.06732400 & 2.33219400 \\
\hline $\mathrm{C}$ & -5.27290000 & 2.33571100 & -0.82244100 \\
\hline $\mathrm{H}$ & -3.61299700 & 2.40800900 & -2.20687700 \\
\hline $\mathrm{H}$ & -6.70377400 & 2.13962800 & 0.78316600 \\
\hline $\mathrm{H}$ & -5.97051700 & 2.81891800 & -1.50090500 \\
\hline $\mathrm{C}$ & -1.58478800 & 1.42418700 & 2.48456000 \\
\hline $\mathrm{H}$ & -0.74532600 & 1.60997400 & 1.80233300 \\
\hline $\mathrm{H}$ & -2.08165000 & 2.36737700 & 2.72512200 \\
\hline $\mathrm{H}$ & -1.19488200 & 0.96454200 & 3.39305300 \\
\hline C & -1.65047900 & 1.20061900 & -0.88165100 \\
\hline O & -1.08749400 & 0.16260700 & -0.42008700 \\
\hline O & -1.16993900 & 2.00866400 & -1.71950000 \\
\hline $\mathrm{Pd}$ & 0.97769100 & -0.35781000 & -0.50403000 \\
\hline $\mathrm{C}$ & 3.49304800 & -0.65878400 & 0.79013100 \\
\hline O & 3.25101800 & -1.07878300 & 1.90762700 \\
\hline O & 4.35898600 & 0.35654900 & 0.54413100 \\
\hline $\mathrm{C}$ & 2.86703700 & -1.16535500 & -0.45929700 \\
\hline $\mathrm{C}$ & 2.42461500 & -2.62589900 & -0.43393900 \\
\hline $\mathrm{H}$ & 3.38685900 & -0.84185600 & -1.36348500 \\
\hline $\mathrm{H}$ & 2.99259000 & -3.25153600 & -1.13240400 \\
\hline
\end{tabular}




2.54031500
4.89612800
4.10021000
5.18349800
6.08347300
5.78649900
6.52649600
6.84933200
1.95807200
1.22413600
3.19023400
4.05678400
3.16869100
3.28230300
1.73270700
0.26014300

2.54031500

$-3.03097900$

1.01334200

1.62471100

0.25767500

1.85011600

2.61552200

2. 35255900

1.21868300

2. 44109100

2. 62206500

3.30065000

2. 65685700

4.11958200

3.68312800

1.60258200

2.18997100
0.57418200

1.71285300

2.15373000

2.44921800

1.27232700

0.54875900

2.13937300

0.81013000

$-0.94961800$

$-2.00157800$

$-0.85007700$

$-1.03465100$

$-1.56997700$

0.17007100

$-0.04980700$

$-1.91002000$

\section{m-TS3}

C

C

C

C

C

C

$\mathrm{H}$

$\mathrm{H}$

$\mathrm{H}$

$\mathrm{H}$

C

$\mathrm{H}$

$\mathrm{H}$

C

$\mathrm{H}$

$\mathrm{H}$

C

O

$\mathrm{N}$

C

C

C

C

C

$\mathrm{H}$
$-0.62961900$

$-0.39727300$

0.88023200

1.95957300

1.71141300

0.42411200

$-1.61709000$

1.05913900

2.53442700

0.24660800

3.36529900

3.40617500

4.05041400

3.86186200

4.94859000

3.67963300

3.22840200

2. 91226400

3.01324000

3.56197600

2.77032900

4. 92698700

3. 37760800

5.51358700

5.52125200
$-2.22963500$

2.33792900

$-2.35707000$

0.96303100

$-2.70776700$

0.51006100

$-2.82758900$

1.38796700

$-2.68934200$

$-2.42114100$

$-1.97648800$

$-2.80591000$

$-2.78048000$

$-2.33032700$

$-2.97653000$

$-3.77216400$

$-3.24397300$

$-1.66862900$

$-1.72568800$

$-0.81715000$

$-1.45062100$

$-2.40753500$

$-0.15706700$

0.97444300

1.79452100

1.24805900

2.85368000

2. 32327300

0.59923500
2.76093200

3.23137100

2. 71147100

$-0.55617400$

3.46587800

4.29934200

0.84336900

0.09273200

1. 65548000

0.18452200

0.03474000

0.84604900

$-1.19618400$

$-1.90331700$

$-1.60361800$

$-0.91733200$

$-0.09196300$

$-1.06096300$

0.59508500

$-0.39445100$

$-1.69840000$ 


\begin{tabular}{|c|c|c|c|}
\hline C & 4.73426100 & 3.12961400 & 0.43773800 \\
\hline $\mathrm{H}$ & 2.76433400 & 3.44934700 & 1.26346600 \\
\hline $\mathrm{H}$ & 6.57406000 & 2.52413400 & -0.51897800 \\
\hline $\mathrm{H}$ & 5.18354600 & 3.96341300 & 0.96984300 \\
\hline $\mathrm{C}$ & 2.43559800 & 0.08110400 & -2.93036300 \\
\hline $\mathrm{H}$ & 1.84354900 & 0.99439900 & -2.89001700 \\
\hline $\mathrm{H}$ & 3.22369900 & 0.16981800 & -3.68924100 \\
\hline $\mathrm{H}$ & 1.79474300 & -0.76312600 & -3.18489900 \\
\hline $\mathrm{C}$ & 1.30000600 & 1.55736500 & 0.14024400 \\
\hline 0 & 0.58052200 & 1.31372800 & -0.88361600 \\
\hline 0 & 0.89706700 & 1.64283100 & 1.32988300 \\
\hline $\mathrm{Pd}$ & -1.22065000 & 0.25137700 & -0.43881900 \\
\hline $\mathrm{C}$ & -2.32049200 & 2.66511900 & 1.16533000 \\
\hline O & -2.43486000 & 1.93428100 & 0.16601900 \\
\hline O & -1.30083600 & 2.69382000 & 1.97166800 \\
\hline $\mathrm{C}$ & -3.42064400 & 3.63188800 & 1.52597900 \\
\hline $\mathrm{H}$ & -4.30965500 & 3.43239100 & 0.92685600 \\
\hline $\mathrm{H}$ & -3.64954500 & 3.55160800 & 2.59268000 \\
\hline $\mathrm{H}$ & -3.07315200 & 4.65436600 & 1.34096800 \\
\hline $\mathrm{C}$ & -3.69312500 & -1.29207100 & -0.81880100 \\
\hline 0 & -3.63928500 & -1.84625500 & -1.90058500 \\
\hline 0 & -4.75691500 & -0.59542000 & -0.37186600 \\
\hline $\mathrm{C}$ & -2.59003300 & -1.25035100 & 0.17695700 \\
\hline $\mathrm{C}$ & -1.41014400 & -2.01020800 & -0.07368300 \\
\hline $\mathrm{H}$ & -2.86001600 & -0.95858000 & 1.18766500 \\
\hline $\mathrm{H}$ & -0.53177400 & -1.05621700 & -1.01062200 \\
\hline $\mathrm{C}$ & -5.85547000 & -0.45668200 & -1.30119400 \\
\hline $\mathrm{H}$ & -5.46772100 & -0.06465100 & -2.24712400 \\
\hline $\mathrm{H}$ & -6.27774400 & -1.44782100 & -1.50018900 \\
\hline $\mathrm{C}$ & -6.86309500 & 0.48045000 & -0.66285700 \\
\hline $\mathrm{H}$ & -6.40785800 & 1.45723300 & -0.46874300 \\
\hline $\mathrm{H}$ & -7.71890600 & 0.62177700 & -1.33174600 \\
\hline $\mathrm{H}$ & -7.22762500 & 0.07352300 & 0.28597700 \\
\hline $\mathrm{H}$ & -1.45150100 & -2.65583200 & -0.95306800 \\
\hline $\mathrm{H}$ & -0.47213600 & 2.14840600 & 1.64525300 \\
\hline
\end{tabular}

\section{INT5}

C
C
C
C
C
C

$$
\begin{array}{r}
-1.14409600 \\
-0.91381300 \\
0.32916200 \\
1.38508200 \\
1.13792600 \\
-0.12106100
\end{array}
$$

2.23656300

1.78276800

0.86986700

2.01761700

0.45765100

2. 52683600

1. 35622900

2. 69318400

2.71191000

2.43874500

3.15026500

2.01472300 
$-2.10566700$

0.50107500

1. 94189400

$-0.28986400$

2.77288900

2.72472600

3. 40427100

3.45061400

4. 52196500

3.36628900

2.86336700

2.34840000

2. 92464100

3.63945300

2. 98628300

4.97936600

3. 68773500

5.67012900

5.46794900

5.02145800

3. 18652800

6.71117400

5.55684400

2.27973500

1.58672200

3.02809200

1. 73920700

1.57549600

0.64994700

1. 44709100

$-1.15875300$

$-1.41675100$

$-1.85333300$

$-0.20760800$

$-2.28685300$

$-3.26737700$

$-2.39273900$

$-1.80471700$

$-3.85128700$

$-3.69326600$

$-4.89366400$

$-2.93601600$

$-1.83118700$

$-3.31013000$
2.57210800

1.40593800

$-0.59694700$

2.71217300

3.43378300

2.56136600

4.20741000

1.83115900

0.85258800

3.04080300

0.15730200

3. 88473200

1. 69742800

3.33798500

0.12838000

1.85379500

0.00558000

2.06396400

0.73519800

0.94940500

$-1.27154600$

1. 63581000

$-1.89567400$

2.56285800

$-1.79403500$

0.36456700

$-1.18663000$

$-0.29416100$

$-1.51663500$

0.27825800

$-0.95915400$

$-2.20993800$

$-0.06347700$

0.99387200

$-1.21966000$

0.37654400

$-3.07614400$

$-2.93621200$

$-3.83154300$

$-3.39943200$

0.12753200

$-0.69616100$

1.23152900

$-0.42552000$

2. 68574000

1.53629500

3.07270500

3.79134600

3.39976600

4.57407400

4.24678600

$-1.29262900$

$-2.36549900$

$-0.99822000$

$-0.12178200$

$-0.20045500$

0.82169700
$-0.71888700$

$-1.58027000$

$-0.94086700$

$-2.64415400$

$-2.01738600$

$-0.26186500$

$-2.87112300$

$-3.28971300$

$-2.18616500$

$-3.70785100$

0.06667000

$-0.76836500$

$-0.20075800$

0.95570400

$-1.25822500$

$-1.57556600$

$-0.68287300$

$-0.52685000$

$-1.60420300$

$-1.46304200$

$-1.29779900$

$-2.14799100$

$-2.41987500$

$-1.38881300$

$-3.01861500$

0.65302400

1.20071400

$-0.15143100$

0.76203200

1. 61190600

0.37897500 


\section{$p$-TS1-cf1}

C

C

C

C

C

C

$\mathrm{H}$

$\mathrm{H}$

$\mathrm{H}$

C

$\mathrm{H}$

$\mathrm{H}$

C

$\mathrm{H}$

$\mathrm{H}$

C

O

$\mathrm{N}$

C

C

C

C

C

$\mathrm{H}$

C

$\mathrm{H}$

$\mathrm{H}$

$\mathrm{H}$

C

$\mathrm{H}$

$\mathrm{H}$

$$
\begin{array}{r}
-0.89944500 \\
-5.83710100 \\
-5.30207500 \\
-6.24656100 \\
-6.90903400 \\
-6.47189100 \\
-7.65172100 \\
-7.41981600 \\
-1.68090400 \\
0.36679700
\end{array}
$$$$
-1.84310900
$$$$
-2.07194000
$$$$
-2.92767400
$$$$
-2.38507800
$$$$
-1.54307200
$$$$
-1.23195600
$$$$
-2.32406700
$$$$
-0.68215400
$$$$
-1.16331800
$$

2.28966800

$-0.03429700$

$-0.37099000$

$-0.79642100$

0.59545300

$-1.30498900$

$-2.25927800$

$-1.50103600$

$-0.86158200$

2.09659400

$-0.97155300$
(

$-0.71631900$

$-1.23523700$

$-2.60611200$

$-3.50524300$

$-3.00082000$

$-1.63130100$

0.47067500

$-0.55369500$

$-2.99564800$

$-4.98323100$

$-5.58041100$

$-5.26309600$

$-5.33412900$

$-5.13186000$

$-6.40362200$

$-4.53584700$

$-4.50214700$

$-3.75999100$

$-3.94422300$

$-2.87031800$

$-5.22733500$

$-3.11108700$

$-5.45896000$

$-6.04033100$

$-4.39524000$

$-2.27419100$

$-6.46569800$

$-4.56259900$

$-2.80378500$

$-2.35521400$

$-2.02178900$
2.33370800

2.56561800

2.65729300

2.51196100

2.38200700

2.29071200

2.96955000

2.63462200

2.76473900

2.34062800

2.79985700

2.80598800

0.83088100

0.36084100

0.71607700

0.18698900

0.71317500

$-0.92788300$

$-1.78008000$

$-2.21407100$

$-2.28425500$

$-3.15157800$

$-3.17386700$

$-1.98433200$

$-3.62053400$

$-3.48807600$

$-3.54120200$

$-4.33161100$

$-1.30604600$

$-2.26657400$

$-0.54767000$
$-0.29232000$

0.99994900

1.22010800

0.15200600

$-1.15185900$

$-1.36988600$

$-0.59723000$

1. 84500800

2.22748200

0.41671600

$-0.37976700$

1.36563200

0.48630300

$-0.47859100$

0.70083300

1. 62049000

2.72804200

1. 35342500

0.23532500

$-0.57801500$

$-0.02209000$

$-1.59625700$

$-1.06845600$

0.63166300

$-1.85460300$

$-2.19766000$

$-1.24669400$

$-2.65765200$

2.40448300

2.14757900

2. 49017400 


\section{$o_{1}$-TS1-cf1}

C

C

C

C

C

C

$\mathrm{H}$

-3.32569700
-1.49440500
-1.31226500
-0.54787100
0.38708200
0.51135700
3.38601000
2.30392900
2.48813400
1.52595100
3.85784900
4.16426200
3.80557200
4.61693900
2.80843500
3.43098800
1.60695300
4.17215500
3.98933300
3.18581000
4.27333200
5.17863700
5.00514700
6.47747000
6.10378100
4.00123100
7.57979100
6.62706000
7.39473500
5.95115500
8.58104100
8.25098300
-3.69409300
-1.25660100

$-1.38888200$

$-1.65498900$

$-0.49574400$

$-2.45754600$

$-2.04394400$

0.60449000

0.44228800

1.43559500

2.71301500

3.56099900

3.25898500

2.88706600

4. 34737200

2.95061400

$-0.94117300$

$-1.75738900$

$-1.15289300$

0.67634700

0.37518700

0.06519800

1.39366500

$-0.54876300$

$-1.91233100$

$-0.06195900$

$-2.76833400$

$-2.30375400$

$-0.91467500$

0.99676900

$-2.27205200$

$-3.82384500$

$-0.51916900$

$-2.93860500$

2.30642900

2.14978100
3.36083800

$-0.46924000$

$-0.04914400$

$-0.88858800$

$-0.80153800$

$-0.30674700$

$-0.60101500$

$-0.64505800$

$-0.90210400$

$-0.89639500$

$-1.25588100$

$-2.24029400$

$-1.28805000$

$-0.53168600$

$-0.97549700$

$-1.61979500$

$-0.44345800$

$-1.32372600$

0.83177000

1.50955700

1.12204200

0.94262900

1. 21258500

0.74802100

1.28689300

1. 35648100

0.82132700

0.54287900

1.09198500

1.49537900

0.67123800

1.15149300

$-1.98761700$

$-2.38147000$
1.38298600

2.60289100

2.88304900

1. 97722200

0.72326300

0.44560400

1.16085300
$-3.18371500$

2.75159000

$-3.58751300$

2.19991600

$-3.35080700$

0.85294000

$-2.66452500$

0.03463200

$-2.27870000$

0.57345000

$-2.56488200$

1.93173800

$-3.38183000$

3.79646400 


$\begin{array}{rrr}3.33072000 & -4.11030000 & 2.81563500 \\ 3.81809600 & -3.71707100 & 0.43410300 \\ -0.20675900 & -2.64298500 & -0.22421400 \\ 2.29121100 & -2.44387800 & -1.43200200 \\ 1.48881500 & -1.86817000 & -1.90218800 \\ 2.32216900 & -3.42050500 & -1.93399300 \\ 3.63318400 & -1.72692300 & -1.71220700 \\ 3.81099100 & -1.75493000 & -2.79207800 \\ 4.46237100 & -2.25347200 & -1.23460700 \\ 3.56342500 & -0.24214300 & -1.36309400 \\ 2.86443000 & 0.49806800 & -2.04504100 \\ 4.29770500 & 0.22448500 & -0.28655800 \\ 4.32660000 & 1.62070100 & 0.00554800 \\ 3.16240300 & 2.39918700 & 0.16265900 \\ 5.57621700 & 2.24027100 & 0.13242200 \\ 3.28256800 & 3.77194600 & 0.42539900 \\ 5.68182100 & 3.59745500 & 0.42950100 \\ 6.47361000 & 1.64890900 & -0.02241900 \\ 4.52983700 & 4.37111300 & 0.57436700 \\ 2.37708600 & 4.36064300 & 0.52057600 \\ 6.66444200 & 4.05040000 & 0.52774600 \\ 4.60076900 & 5.43232600 & 0.79257100 \\ 5.11625100 & -0.64987500 & 0.56273600 \\ 4.52505400 & -1.49360400 & 0.91999300 \\ 6.01005100 & -1.01890500 & 0.04463100 \\ 5.43444900 & -0.07954300 & 1.43573100 \\ 1.79176100 & 1.82564200 & 0.07383700 \\ 1.54408000 & 0.67460900 & 0.49315400 \\ 0.91371800 & 2.65960300 & -0.41443200 \\ -0.25110900 & -0.40411800 & 0.03085900 \\ -3.08525300 & -0.17032800 & -0.61651000 \\ -2.01377400 & -1.16852400 & -0.56902100 \\ -2.14864800 & -2.40826400 & -1.01407300 \\ -1.20039200 & -3.25306200 & -0.95814300 \\ -3.45620100 & -2.87600600 & -1.63026800 \\ -3.61306500 & -2.38656500 & -2.59832600 \\ -3.40244000 & -3.95420700 & -1.78239900 \\ -4.31332700 & -2.63847700 & -0.99326000 \\ -2.47475400 & 1.22839000 & -0.82524600 \\ -3.08823300 & 2.13831600 & -1.34074300 \\ -1.25749900 & 1.35267300 & -0.30634300 \\ -3.76261200 & -0.35443000 & -1.45451500 \\ -3.89937200 & -0.18992600 & 0.71242700 \\ & -.16476500 & 1.51400400\end{array}$

$-4.11030000$

$-3.71707100$

$-2.64298500$

$-3.42050500$

$-1.75493000$

$-2.25347200$

$-0.24214300$

0.49806800

2.24027100

3.77194600

3.59745500

4.05040000

5.43232600

$-0.07954300$

1.82564200

0.67460900

0.16476500
2.81563500

0300

$-1.43200200$

18800

$-1.71220700$

$-79207800$

$-1.36309400$

$-2.04504100$

0.00554800

0.16265900

0.42539900

0.42950100

0.02241900

0.52057600

0.79257100

0.56273600

0.91999300

0.04463100

0.07383700

0.49315400

0.41443200

$-0.61651000$

.56902100

$-1.01407300$

$-0.95814300$

$-2.59832600$

$-1.78239900$

$-0.99326000$

$-0.82524600$

$-1.34074300$

$-0.30634300$

0.71242700

1. 51400400 
$\mathrm{H}$

C

C

C

C

$\mathrm{H}$

C

$\mathrm{H}$

C

$\mathrm{H}$

$\mathrm{H}$

$\mathrm{H}$

$\mathrm{H}$

$\mathrm{H}$
$-4.12760600$

$-5.17632000$

$-5.19207400$

$-6.37140200$

$-6.37356700$

$-4.27019700$

$-7.55556100$

$-6.37495900$

$-7.55909300$

$-6.36706000$

$-8.47379300$

$-8.47952500$

$-0.02803200$

$-0.53343400$
$-1.23944500$

0.61443200

1.97595800

0.00881300

2.71309600

2.46230700

0.74237000

$-1.05058900$

2.09901000

3.76925900

0.25472400

2. 67363100

2.27051100

$-2.31181300$
0.93428800

0.65756300

0.98503600

0.24743200

0.90597500

1.29126100

0.16502000

$-0.00432400$

0.49582000

1.16165800

$-0.15214700$

0.43506500

$-0.43810300$

2.33112100

\section{m-TS1-cf2}

$\begin{array}{ll}\mathrm{C} & -0.44410800 \\ \mathrm{C} & -1.52573200 \\ \mathrm{C} & -2.81758300 \\ \mathrm{C} & -3.04916800 \\ \mathrm{C} & -1.94975300 \\ \mathrm{C} & -0.63647300 \\ \mathrm{H} & 0.56375700 \\ \mathrm{H} & -1.37530800 \\ \mathrm{H} & -3.66815400 \\ \mathrm{H} & 0.32908000 \\ \mathrm{C} & -4.46108200 \\ \mathrm{H} & -4.59306200 \\ \mathrm{H} & -5.17509300 \\ \mathrm{C} & -4.79340500 \\ \mathrm{H} & -4.19011300 \\ \mathrm{H} & -5.84536400 \\ \mathrm{C} & -4.54274500 \\ \mathrm{O} & -4.73555500 \\ \mathrm{~N} & -4.01815300 \\ \mathrm{C} & -4.09605600 \\ \mathrm{C} & -2.95365500 \\ \mathrm{C} & -5.35984900 \\ \mathrm{C} & -3.11362800 \\ \mathrm{C} & -5.50788600 \\ \mathrm{H} & -6.22581300 \\ \mathrm{C} & -4.37888100 \\ \mathrm{H} & -2.23015200\end{array}$

3.02954900

3.58620300

3.41488200

2. 72865500

2.21204200

2. 32261100

3.14674200

4.13086300

3.79135600

2. 66072900

2.43492500

2.72302700

3. 01192400

0.93224100

0.34756600

0.75920100

0.46872300

1.21838900

$-0.79887100$

$-1.79975500$

$-2.35829500$

$-2.30229800$

$-3.41944700$

$-3.32995000$

$-1.88022200$

$-3.89855700$

$-3.84823000$
1.00475400

1.68068800

1.17743400

$-0.02431400$

$-0.71082500$

$-0.20500500$

1.39592800

2. 60910700

1.73743500

$-1.06881600$

$-0.48938300$

$-1.53981600$

0.10450700

$-0.35325800$

$-1.05345500$

$-0.61462500$

1.08257800

2.03321800

1.24445700

0.23330300

$-0.38220400$

$-0.09659400$

$-1.28852300$

$-1.02724000$

0.40452000

$-1.61806400$

$-1.74728700$ 
$\mathrm{H}$

$\mathrm{H}$

C

$\mathrm{H}$

$\mathrm{H}$

$\mathrm{H}$

C

O

O

$\mathrm{H}$

$\mathrm{Pd}$

C

$\mathrm{N}$

C

O

C

$\mathrm{H}$

$\mathrm{H}$

$\mathrm{H}$

C

O

O

$\mathrm{H}$

C

$\mathrm{H}$

$\mathrm{H}$

C

C

C

C

$\mathrm{H}$

C

$\mathrm{H}$

C

$\mathrm{H}$

$\mathrm{H}$

$\mathrm{H}$

$\mathrm{H}$
$-6.50054500$

$-4.48064100$

$-3.51760900$

$-3.58858100$

$-4.11414900$

$-2.47192200$

$-1.58157000$

$-1.41198400$

$-0.62343200$

0.31285700

0.43682800

3. 29661100

2. 20611800

2. 32979500

1. 34812300

3.66029900

3. 90161400

3.58342400

4.47529800

2.72008100

3.34740100

1. 51326300

4.04115600

3.98163600

3.22683600

4.26123200

5.19477400

5.07216800

6.46399100

6.19180100

4.09100100

7.58689900

6.57352000

7.45272900

6.07876900

8.56479400

8.32524700

$-2.11167500$
$-3.69644500$

$-4.70994000$

$-1.21104400$

$-0.35179400$

$-2.03717000$

$-1.52457200$

$-1.80517300$

$-0.61429300$

$-2.65623800$

$-2.23591100$

0.47600200

0.22992400

1.19562600

2.33349000

3.13148500

2. 75137900

2.11609700

3. 78597900

2.65988600

$-1.20162100$

$-2.13566400$

$-1.30162500$

0.31415100

0.46040600

0.29401200

1.52005800

$-0.41040200$

$-1.67889800$

0.02545800

$-2.49175600$

$-2.03282100$

$-0.78452600$

1.01144000

$-2.04699000$

$-3.47411800$

$-0.42917000$

$-2.67988900$

1.66676400
$-1.27314400$

$-2.33236300$

2. 55547300

3.22163000

2. 95942200

2.47446300

$-0.19615300$

0.13410400

$-0.46137200$

$-0.39888300$

$-0.27368200$

$-0.70965200$

$-0.89465700$

$-1.55206500$

$-1.72960300$

$-2.15089300$

$-3.01079300$

$-2.48580300$

$-1.42697500$

$-0.77925300$

$-1.23024800$

$-0.22817300$

$-1.50517800$

0.66666700

1. 44412500

0.71652100

0.89130700

1. 47234400

0.48988900

1.64893100

1.77866100

0.66360400

0.04134300

1.24529800

2.09958300

0.34913700

1.38371400

$-1.63865700$

\section{m-TS1-cf3}

$\mathrm{C}$
$\mathrm{C}$
$\mathrm{C}$
0.22836300

1.17144100

2.50468400
$-2.88679700$

$-3.53284700$

$-3.57427900$
1.17641400

1.97147600

1.55811900 
2.91867800

1. 96271300

0.60691600

$-0.81870900$

0.87467700

3.24221400

2. 27483200

$-0.25943800$

4. 38181800

4.50438700

4.84662200

5.14346100

6.21753600

4.97214100

4.80984600

5.18623000

4.11393200

4.10960500

2.93727900

5.34786200

3.04960800

5.44265300

6.23994200

4.28411900

2.14741100

6.41706500

4.34340800

3.48892200

2.88829300

4.24025500

2. 82076700

1.58658100

1. 41057800

0.63065600

$-0.40968100$

$-3.28055000$

$-2.17396600$

$-2.26807500$

$-1.26702200$

$-3.59188100$

$-3.85415000$

$-3.49016600$

$-4.40511200$

$-2.73826100$
$-2.97573500$

$-2.32083500$

$-2.26397300$

$-2.88396400$

$-4.01446200$

$-4.08629500$

$-1.84158300$

$-2.55185400$

$-2.97524000$

$-2.87717000$

$-3.92207300$

$-1.81331000$

$-1.94782000$

$-1.82928600$

$-0.47384700$

$-0.25476600$

0.46887900

1.81208800

2.52181500

2.46004600

3.87370200

3.78706300

1.89840100

4.50386800

4.41567900

4.26373700

5.54439200

0.19087900

$-0.71861300$

0.10633600

1.01549000

1.90107800

0.68334200

2.73960500

$-0.39256600$

$-0.21047900$

$-1.15637600$

$-2.30872300$

$-3.07813500$

$-2.76587400$

$-2.14714200$

$-3.80200000$

$-2.68668700$

1.22950200
0.35685900

$-0.42310100$

$-0.03512200$

1. 46862700

2.89950500

2.17369500

$-1.34771500$

$-0.96593600$

$-0.02297900$

$-1.10660100$

0.27654600

0.65304400

0.48378800

1.73274600

$-0.00238300$

$-1.14809600$

0.72866900

0.24053400

$-0.10389400$

0.14955700

$-0.48801200$

$-0.25366300$

0.40444300

$-0.56353500$

$-0.74251900$

$-0.31307000$

$-0.86799600$

2.02590700

1. 98093400

2.82169400

2. 27186500

$-0.15397700$

0.07205800

$-0.46998400$

$-0.32603500$

$-0.73194000$

$-0.89734600$

$-1.54111300$

$-1.70302200$

$-2.12765200$

$-2.99344400$

$-2.45106000$

$-1.39994300$

$-0.84850400$ 
$-3.41951000$

$-1.48962200$

$-4.03201000$

$-3.95070600$

$-3.20166600$

$-4.17918900$

$-5.20401500$

$-5.14623300$

$-6.44951900$

$-6.30567100$

$-4.18481600$

$-7.61205500$

$-6.50902100$

$-7.54221600$

$-6.24268200$

$-8.57038200$

$-8.44556800$

$-0.29373500$
2.15370000

1. 35575300

$-0.33408000$

$-0.41596900$

$-0.18664900$

$-1.48527900$

0.40195300

1.68855100

$-0.10684400$

2.44779000

2.09998400

0.64942300

$-1.10798300$

1.93059300

3.44541800

0.23768400

2.52200400

2.30674100
$-1.23642300$

$-0.40878900$

$-1.51588900$

0.65691100

1.42439800

0.74675200

0.85755100

1.40739200

0.46727200

1. 56481700

1.70368800

0.62110900

0.04338200

1.17234600

1. 99102300

0.31533900

1.29556100

$-0.49801900$

\section{m-TS1-cf4}

$\begin{array}{ll}\mathrm{C} & -1.25295800 \\ \mathrm{C} & -0.87796700 \\ \mathrm{C} & -1.88113600 \\ \mathrm{C} & -3.23074900 \\ \mathrm{C} & -3.56246700 \\ \mathrm{C} & -2.58395500 \\ \mathrm{H} & -0.48614100 \\ \mathrm{H} & 0.32079000 \\ \mathrm{H} & -1.61776800 \\ \mathrm{H} & -4.60771600 \\ \mathrm{H} & -2.86770800 \\ \mathrm{C} & -4.32224900 \\ \mathrm{H} & -4.02295600 \\ \mathrm{H} & -5.23384100 \\ \mathrm{C} & -4.65066000 \\ \mathrm{H} & -5.58426300 \\ \mathrm{H} & -4.81068400 \\ \mathrm{C} & -3.55437000 \\ \mathrm{O} & -2.96431900 \\ \mathrm{~N} & -3.23626100 \\ \mathrm{C} & -3.77410100 \\ \mathrm{C} & -2.96922000 \\ \mathrm{C} & -5.08627400\end{array}$

2.89316100

2.44517500

2.30674800

2.52749400

2.96850300

3.17397200

3.03252100

3. 00924100

1.95746200

3.15083100

3.53399800

2.18024000

2.44100500

2.74116100

0.66979200

0.48399300

0.34129900

$-0.14187000$

0.29793900

$-1.39286400$

$-1.97335100$

$-2.17222800$

$-2.45336600$
$-1.53623600$

$-0.24831400$

0.73389100

0.43521200

$-0.85445400$

$-1.83104800$

$-2.29471900$

0.05428800

1. 72809800

$-1.09798100$

$-2.81678200$

1.42609900

2.44529700

1.19009600

1.39086000

1.93957600

0.36038400

2.07920500

3.06148400

1.59194700

0.40588800

$-0.73719900$

0.40098300 


\begin{tabular}{|c|c|c|c|}
\hline $\mathrm{C}$ & -3.47273000 & -2.88097600 & -1.83420300 \\
\hline $\mathrm{C}$ & -5.59988400 & -3.11018900 & -0.71863200 \\
\hline $\mathrm{H}$ & -5.68959000 & -2.32130000 & 1.29375500 \\
\hline $\mathrm{C}$ & -4.78964000 & -3.33840700 & -1.83269300 \\
\hline $\mathrm{H}$ & -2.82942600 & -3.04585000 & -2.69253900 \\
\hline $\mathrm{H}$ & -6.62519700 & -3.46885400 & -0.70690800 \\
\hline $\mathrm{H}$ & -5.17947000 & -3.87093500 & -2.69483100 \\
\hline $\mathrm{C}$ & -2.08974900 & -2.05312200 & 2.23312700 \\
\hline $\mathrm{H}$ & -1.17068300 & -1.48233000 & 2.06417100 \\
\hline $\mathrm{H}$ & -1.98622000 & -3.05726600 & 1.81822200 \\
\hline $\mathrm{H}$ & -2.26077400 & -2.11482400 & 3.30992900 \\
\hline C & -1.61434600 & -1.56408600 & -0.82150400 \\
\hline 0 & -1.44836800 & -0.41777100 & -0.36625400 \\
\hline 0 & -0.69590700 & -2.29218500 & -1.40043400 \\
\hline $\mathrm{H}$ & 0.23095800 & -1.85693000 & -1.33177800 \\
\hline $\mathrm{Pd}$ & 0.35148100 & 0.70367600 & -0.35789500 \\
\hline $\mathrm{C}$ & 3.23648000 & 0.54334600 & -0.49670100 \\
\hline $\mathrm{N}$ & 2.16834000 & 1.54198200 & -0.35007600 \\
\hline $\mathrm{C}$ & 2.38182600 & 2.82524100 & -0.13585200 \\
\hline 0 & 1.42986500 & 3.64657100 & 0.10028800 \\
\hline $\mathrm{C}$ & 3.77930200 & 3.41146100 & -0.20344300 \\
\hline $\mathrm{H}$ & 4.16788200 & 3.33044500 & -1.22497400 \\
\hline $\mathrm{H}$ & 3.73458000 & 4.46292600 & 0.08047700 \\
\hline $\mathrm{H}$ & 4.47272800 & 2.88473500 & 0.45777000 \\
\hline C & 2.66347800 & -0.69420800 & -1.22254600 \\
\hline O & 3.30139300 & -1.34329300 & -2.02251100 \\
\hline 0 & 1.44005700 & -1.00720200 & -0.79725100 \\
\hline $\mathrm{H}$ & 4.06516700 & 0.93106200 & -1.09605200 \\
\hline $\mathrm{C}$ & 3.75445300 & 0.09705900 & 0.90342000 \\
\hline $\mathrm{H}$ & 2.91664300 & -0.38262700 & 1.42175500 \\
\hline $\mathrm{H}$ & 4.00388200 & 0.99815700 & 1.47470800 \\
\hline $\mathrm{C}$ & 4.95036000 & -0.82268600 & 0.83630900 \\
\hline $\mathrm{C}$ & 4.78480300 & -2.20920900 & 0.72428500 \\
\hline $\mathrm{C}$ & 6.24988900 & -0.29999200 & 0.85245600 \\
\hline $\mathrm{C}$ & 5.89165300 & -3.05279500 & 0.63145700 \\
\hline $\mathrm{H}$ & 3.78099800 & -2.62552500 & 0.70082600 \\
\hline C & 7.36038200 & -1.14010000 & 0.76023200 \\
\hline $\mathrm{H}$ & 6.39283300 & 0.77547300 & 0.94362300 \\
\hline $\mathrm{C}$ & 7.18297300 & -2.52083000 & 0.64959500 \\
\hline $\mathrm{H}$ & 5.74536100 & -4.12594900 & 0.54298300 \\
\hline $\mathrm{H}$ & 8.36179800 & -0.71785200 & 0.77813800 \\
\hline & 8.04552400 & -3.17811800 & 0.57880200 \\
\hline
\end{tabular}




\begin{tabular}{|c|c|c|c|}
\hline $\mathrm{C}$ & 0.71339800 & -2.18191500 & 1.96216200 \\
\hline $\mathrm{C}$ & 1.90113400 & -2.42005500 & 2.64791100 \\
\hline $\mathrm{C}$ & 3.07518200 & -2.67484300 & 1.93359900 \\
\hline $\mathrm{C}$ & 3.08013500 & -2.73500700 & 0.53289800 \\
\hline $\mathrm{C}$ & 1.87793300 & -2.49644400 & -0.13809300 \\
\hline $\mathrm{C}$ & 0.68360000 & -2.19500800 & 0.54914300 \\
\hline $\mathrm{H}$ & -0.20156500 & -1.97987100 & 2.51404000 \\
\hline $\mathrm{H}$ & 1.92541000 & -2.39411000 & 3.73422300 \\
\hline $\mathrm{H}$ & 4.00946400 & -2.82142500 & 2.46920500 \\
\hline $\mathrm{H}$ & 1.85674200 & -2.52907500 & -1.22593700 \\
\hline $\mathrm{H}$ & -0.38615600 & -2.81930100 & 0.03135400 \\
\hline $\mathrm{C}$ & 4.37050000 & -2.97467600 & -0.22671100 \\
\hline $\mathrm{H}$ & 4.31057000 & -3.90544300 & -0.80548400 \\
\hline $\mathrm{H}$ & 5.19756900 & -3.07827500 & 0.48049700 \\
\hline $\mathrm{C}$ & 4.69013600 & -1.81629700 & -1.19306300 \\
\hline $\mathrm{H}$ & 3.95281100 & -1.79162700 & -1.99638600 \\
\hline $\mathrm{H}$ & 5.66857000 & -1.98528400 & -1.66181500 \\
\hline C & 4.76726000 & -0.50308000 & -0.41247600 \\
\hline O & 5.35720000 & -0.46091500 & 0.65960400 \\
\hline $\mathrm{N}$ & 4.14116200 & 0.60649600 & -0.95543700 \\
\hline $\mathrm{C}$ & 4.10983700 & 1.82078700 & -0.20462000 \\
\hline $\mathrm{C}$ & 2.89353500 & 2.47732700 & 0.07930500 \\
\hline $\mathrm{C}$ & 5.31225000 & 2.41616500 & 0.18984400 \\
\hline $\mathrm{C}$ & 2.91254700 & 3.74186300 & 0.69109000 \\
\hline $\mathrm{C}$ & 5.31470100 & 3.64889900 & 0.83820500 \\
\hline $\mathrm{H}$ & 6.24085600 & 1.90120400 & -0.02317100 \\
\hline $\mathrm{C}$ & 4.11463300 & 4.32345600 & 1.07848200 \\
\hline $\mathrm{H}$ & 1.97002600 & 4.24475600 & 0.87791600 \\
\hline $\mathrm{H}$ & 6.25980600 & 4.09294100 & 1.13792200 \\
\hline $\mathrm{H}$ & 4.11627100 & 5.29335700 & 1.56660400 \\
\hline C & 3.45325300 & 0.57433700 & -2.25208400 \\
\hline $\mathrm{H}$ & 3.18346300 & 1.59673500 & -2.52288500 \\
\hline $\mathrm{H}$ & 4.11803600 & 0.19125300 & -3.03180800 \\
\hline $\mathrm{H}$ & 2.53858300 & -0.02525700 & -2.21819900 \\
\hline $\mathrm{C}$ & 1.57245500 & 1.84395700 & -0.17203700 \\
\hline O & 1.43325600 & 0.61007700 & -0.05742600 \\
\hline O & 0.61403100 & 2.68453100 & -0.47896000 \\
\hline $\mathrm{Pd}$ & -0.38597400 & -0.50801700 & -0.21829900 \\
\hline $\mathrm{H}$ & -0.27272700 & 2.21620100 & -0.65190500 \\
\hline $\mathrm{C}$ & -3.26203100 & -0.36276500 & -0.60940100 \\
\hline $\mathrm{N}$ & -2.18419800 & -1.34850800 & -0.47416300 \\
\hline $\mathrm{C}$ & -2.36791500 & -2.65216300 & -0.55923800 \\
\hline 0 & -1.41865500 & -3.48734400 & -0.37095900 \\
\hline $\mathrm{C}$ & -3.72485800 & -3.23329100 & -0.90978800 \\
\hline
\end{tabular}




\section{m-TS1-cf6}

C

C

C

C

C

C

$\mathrm{H}$

$\mathrm{H}$

$\mathrm{H}$

$\mathrm{H}$

C

$\mathrm{H}$

$\mathrm{H}$

C

$\mathrm{H}$

$\mathrm{H}$

C

O

$\mathrm{N}$

C

$$
\begin{aligned}
& -3.99863000 \\
& -3.67299700 \\
& -4.50839200 \\
& -2.69561900 \\
& -3.40437700 \\
& -1.40785800 \\
& -4.01204500 \\
& -3.95007100 \\
& -3.24181400 \\
& -4.09465800 \\
& -5.27322900 \\
& -5.34522900 \\
& -6.46037400 \\
& -6.57378300 \\
& -4.43176800 \\
& -7.69165700 \\
& -6.42063500 \\
& -7.75080100 \\
& -6.61101900 \\
& -8.60237700 \\
& -8.70766700
\end{aligned}
$$$$
-
$$$$
.96094600
$$$$
-4.31919900
$$$$
-2.85583100
$$$$
0.98197200
$$$$
1.81896500
$$$$
1.15827400
$$$$
-0.69380800
$$$$
-0.12952900
$$$$
0.41058700
$$$$
-1.11436000
$$$$
0.59421900
$$

1.99144400

$-0.13285300$

2.64492700

2.56903500

0.51635900

$-1.22035400$

1. 91004000

3.73000000

$-0.06469100$

2.41998100
$-1.93536800$

$-0.83000200$

$-0.24610000$

$-1.11373200$

$-1.63325100$

$-0.85136200$

$-1.33338800$

0.77276300

1.41293800

1.23196700

0.68889900

0.74853400

0.52895700

0.65270300

0.85375200

0.43033200

0.48877600

0.49349900

0.69857000

0.31021100

0.41976800
$-1.45972400$

$-0.81958800$

$-1.60360500$

$-2.98567200$

$-3.58682800$

$-2.83018500$

$-0.86905700$

0.38601700

$-1.12124300$

$-4.66533000$

$-3.83169300$

$-4.86297300$

$-3.45794300$

$-3.82249900$

$-4.50671300$

$-2.82204200$

$-4.30464200$

$-5.27269400$

$-3.59173300$

$-3.90349500$
2.49571100

2. 34836900

2.47382600

2.67377800

2.78529100

2.72367100

2.42909300

2.96073500

2. 37762700

2.90054000

2.64114200

2.91437000

3. 35795900

1.23183700

1.20334700

1.01310100

0.20551400

0.45315400

$-0.97656400$

$-1.87899100$
$-1.69330500$

$-0.44111000$

0.72503900

0.66690200

$-0.59493800$

$-1.76829300$

$-2.60401800$

$-0.44585200$

1.69678100

$-0.65346700$

1.92348900

1.68569900

2. 66521600

2.55653400

3.41494600

2.93302400

1. 52913600

0.82066600

1.41945300

0.35679600 


\begin{tabular}{|c|c|c|c|}
\hline C & -2.91802000 & -2.30914800 & -0.55928700 \\
\hline C & -5.19590700 & -2.40454000 & 0.26119500 \\
\hline C & -3.23496600 & -3.29802000 & -1.50576300 \\
\hline $\mathrm{C}$ & -5.50788200 & -3.35149300 & -0.71159200 \\
\hline $\mathrm{H}$ & -5.94791300 & -2.06207400 & 0.96114400 \\
\hline C & -4.52409200 & -3.81204500 & -1.59053500 \\
\hline $\mathrm{H}$ & -2.46029200 & -3.63499700 & -2.18568100 \\
\hline $\mathrm{H}$ & -6.51900100 & -3.74465600 & -0.76948700 \\
\hline $\mathrm{H}$ & -4.76114200 & -4.56321900 & -2.33787300 \\
\hline C & -2.48318400 & -1.31720900 & 2.31923400 \\
\hline $\mathrm{H}$ & -1.60262400 & -0.69224800 & 2.14449400 \\
\hline $\mathrm{H}$ & -2.79723900 & -1.23006000 & 3.36360300 \\
\hline $\mathrm{H}$ & -2.20705000 & -2.35854800 & 2.14289600 \\
\hline $\mathrm{C}$ & -1.56434100 & -1.69792100 & -0.61663200 \\
\hline O & -1.40128900 & -0.50296700 & -0.29654200 \\
\hline O & -0.61581500 & -2.49362700 & -1.04494000 \\
\hline $\mathrm{Pd}$ & 0.41281400 & 0.60773800 & -0.41482900 \\
\hline $\mathrm{C}$ & 3.30560000 & 0.44505100 & -0.55005600 \\
\hline $\mathrm{N}$ & 2.22991000 & 1.44556000 & -0.55841700 \\
\hline C & 2.43915600 & 2.74476900 & -0.63577000 \\
\hline O & 1.48652500 & 3.59647600 & -0.57365500 \\
\hline $\mathrm{C}$ & 3.83254200 & 3.30777200 & -0.84475200 \\
\hline $\mathrm{H}$ & 4.19378600 & 3.04261900 & -1.84499700 \\
\hline $\mathrm{H}$ & 3.78974000 & 4.39357900 & -0.75811400 \\
\hline $\mathrm{H}$ & 4.54614700 & 2.91102700 & -0.11751300 \\
\hline $\mathrm{C}$ & 2.74272700 & -0.90353100 & -1.04783900 \\
\hline O & 3.39825400 & -1.68619400 & -1.70089200 \\
\hline O & 1.51060200 & -1.13746900 & -0.60215000 \\
\hline $\mathrm{H}$ & 4.12055700 & 0.73119600 & -1.22063800 \\
\hline $\mathrm{C}$ & 3.85444500 & 0.25469200 & 0.89512400 \\
\hline $\mathrm{H}$ & 3.03167800 & -0.13007600 & 1.50839200 \\
\hline $\mathrm{H}$ & 4.10735900 & 1.24510300 & 1.29109400 \\
\hline $\mathrm{C}$ & 5.05764100 & -0.65505800 & 0.96616300 \\
\hline $\mathrm{C}$ & 4.90596300 & -2.03816200 & 1.12655300 \\
\hline $\mathrm{C}$ & 6.35053700 & -0.13099700 & 0.83973900 \\
\hline $\mathrm{C}$ & 6.01986200 & -2.87694600 & 1.16122800 \\
\hline $\mathrm{H}$ & 3.90741400 & -2.45861700 & 1.21439100 \\
\hline $\mathrm{C}$ & 7.46795400 & -0.96615600 & 0.87285000 \\
\hline $\mathrm{H}$ & 6.48303000 & 0.94293500 & 0.71896800 \\
\hline $\mathrm{C}$ & 7.30444200 & -2.34354200 & 1.03476100 \\
\hline $\mathrm{H}$ & 5.88403100 & -3.94811200 & 1.28379700 \\
\hline $\mathrm{H}$ & 8.46401500 & -0.54194500 & 0.77616500 \\
\hline $\mathrm{H}$ & 8.17240600 & -2.99688500 & 1.06236200 \\
\hline $\mathrm{H}$ & 0.30568000 & -2.05196500 & -1.00682400 \\
\hline
\end{tabular}




\section{m-TS1-cf7}

$C$
$C$
$C$
$C$
$C$

C

C

C

C

C

$\mathrm{H}$

$\mathrm{H}$

$\mathrm{H}$

$\mathrm{H}$

$\mathrm{H}$

C

$\mathrm{H}$

$\mathrm{H}$

C

$\mathrm{H}$

$\mathrm{H}$

C

O

$\mathrm{N}$

C

C

C

C

C

$\mathrm{H}$

C

$\mathrm{H}$

$\mathrm{H}$

$\mathrm{H}$

C

$\mathrm{H}$

$\mathrm{H}$

$\mathrm{H}$

C

O

O

$\mathrm{Pd}$

$\mathrm{H}$

C
$-1.05954600$

$-0.87642600$

$-1.98935800$

$-3.24897900$

$-3.38787600$

$-2.30691300$

$-0.20224600$

0.23424100

$-1.86928700$

$-4.36407800$

$-2.43967900$

$-4.46723900$

$-5.08222000$

$-4.17804000$

$-5.33260500$

$-5.56733100$

$-6.27791800$

$-4.68581200$

$-4.62315100$

$-4.22031700$

$-3.88103300$

$-2.60881300$

$-4.89304800$

$-2.40928000$

$-4.67029100$

$-5.86295200$

$-3.42550600$

$-1.43310700$

$-5.47371000$

$-3.24671500$

$-4.06720100$

$-5.04093600$

$-3.42486900$

$-3.60533500$

$-1.42139700$

$-1.39206800$

$-0.42707000$

0.39073200

0.44746400

3.31054600
$-3.07333700$

$-2.39316000$

$-2.23238400$

$-2.72477800$

$-3.40855000$

$-3.58295700$

$-3.23281700$

$-2.80620800$

$-1.70027200$

$-3.80535200$

$-4.12097300$

$-2.45956300$

$-3.36510400$

$-2.17838900$

$-1.32223400$

$-1.53688800$

$-1.27459500$

0.04606300

0.50884700

0.74228700

2.11831000

2. 64551400

2.98846000

4.03844900

4.35755200

2.56994200

4.88996400

4.43486200

5.01001400

5.95926800

0.14182900

$-0.12332800$

$-0.73918900$

0.87804800

1.79880100

0.58695300

2.44858100

$-0.65084600$

1.91829300

$-0.62799400$
1.26141100

0.03632800

$-0.81679900$

$-0.47174000$

0.74788000

1.61370200

1.91019100

$-0.48112300$

$-1.75716400$

1.02112000

2.54862400

$-1.32626100$

$-1.39716100$

$-2.34403900$

$-0.73668900$

0.30935900

$-1.28811200$

$-0.95049200$

$-2.08305500$

0.15034100

$-0.02161300$

0.28833000

$-0.44477100$

0.21587400

$-0.54355000$

$-0.68890000$

$-0.19707300$

0.46751900

$-0.87382200$

$-0.25636500$

1. 48060400

1.90885800

1.44836500

2.13896600

0.58132300

0.27546100

1.13437500

0.20695500

1.11722300

0.36449400 
2.19520600

2. 35185700

1.37148000

3. 71541000

4.50687100

3.62554600

3.98897700

2. 77507600

3. 42190200

1.57411500

4.07075300

3.95132500

3.17869900

4.19478500

5.19012600

5.10292700

6.45208100

6.24919400

4.12869000

7. 60161400

6.53488700

7. 50221200

6.16319500

8.57300300

8.39549700
$-1.55226800$

$-2.78015700$

$-3.49726000$

$-3.43840500$

$-2.91401500$

$-4.45798700$

$-3.50821700$

0.60708300

1.21655700

0.97902500

$-1.09257200$

$-0.14532900$

0.40527000

$-1.03070900$

0.69705100

2.08781500

0.09241800

2. 85580200

2.56807300

0.85648600

$-0.98713200$

2. 24250400

3. 93358900

0.37110900

2. 84090500
0.10327500

$-0.36935100$

$-0.74749500$

$-0.47938000$

0.05735800

$-0.09625600$

$-1.53738800$

1.11150700

1.93418800

0.67037900

0.99900400

$-0.97154900$

$-1.52018700$

$-1.56816800$

$-0.77965100$

$-0.63884200$

$-0.71071400$

$-0.43455600$

$-0.68064700$

$-0.50554100$

$-0.82472900$

$-0.36719100$

$-0.32570000$

$-0.45787100$

$-0.20918200$

\section{m-TS1-cf8}

C

C

C

C

C

C

$\mathrm{H}$

$\mathrm{H}$

$\mathrm{H}$

$\mathrm{H}$

C

$\mathrm{H}$

$\mathrm{H}$

C

$\mathrm{H}$

$\mathrm{H}$
$-1.23193400$

$-2.55788600$

$-3.50693900$

$-3.14483900$

$-1.79920300$

$-0.82393500$

$-0.48820000$

$-2.86384700$

$-4.55284000$

0.36179900

$-4.20609700$

$-3.88763100$

$-5.13548900$

$-4.49279100$

$-3.61314300$

$-5.31841000$
$-3.14185400$

$-3.53490000$

$-3.14287000$

$-2.38657400$

$-2.05736200$

$-2.39547600$

$-3.43215900$

$-4.12589100$

$-3.39056000$

$-2.90124000$

$-1.81621500$

$-1.87389400$

$-2.38397900$

$-0.33148800$

0.27972900

0.03534900
0.91658100

1.06819600

0.12136200

$-1.00529200$

$-1.17673200$

$-0.21362100$

1. 65484300

1.92757300

0.27032000

$-0.57886200$

$-1.92296800$

$-2.97077500$

$-1.82408600$

$-1.58230900$

$-1.80331200$

$-2.20501500$ 


$$
\begin{aligned}
& -4.87437800 \\
& -5.59991200 \\
& -4.26911600 \\
& -3.89451400 \\
& -2.58087200 \\
& -4.88828600 \\
& -2.30992400 \\
& -4.59713700 \\
& -5.90086800 \\
& -3.30631100 \\
& -1.30012000 \\
& -5.38323400 \\
& -3.07342600 \\
& -4.40401600 \\
& -4.39515000 \\
& -5.34054800 \\
& -3.56178900 \\
& -1.42431900 \\
& -1.40092600 \\
& -0.45552100 \\
& 0.40013200 \\
& 0.41177200 \\
& -1.50599300 \\
& 3.32171800 \\
& 2.22878100 \\
& 2.42866900 \\
& 1.47488900 \\
& 3.81783000 \\
& 4.21761100 \\
& 3.75261500 \\
& 4.51453000 \\
& 2.79342500 \\
& 3.49234000 \\
& 1.54333000 \\
& 4.13297700 \\
& 3.87283700 \\
& 3.07467400 \\
& 4.05032000 \\
& 5.13977800 \\
& 5.09535700 \\
& 6.38895600 \\
& 6.27056100 \\
& 4.13260300 \\
& 7.56740400
\end{aligned}
$$

$-0.21240900$

$-1.03955000$

0.79596900

2.03756900

2.54630100

2.81774700

3.83502400

4.07896200

2.42647800

4.59580600

4.22059600

4.66276200

5.58428500

0.76598300

$-0.27394600$

1.23373600

1.30070600

1.72782000

0.49843100

2.42864000

1.88363600

$-0.71866600$

$-1.47597000$

$-0.62521200$

$-1.56608800$

$-2.80028200$

$-3.55399800$

$-3.41259000$

$-3.44656500$

$-4.42610300$

$-2.83007700$

0.53744900

1.13441800

0.86898700

$-1.11344200$

$-0.01347300$

0.60118400

$-0.83448200$

0.78756800

2.16116900

0.15684400

2.88708300

2.66033300

0.87818200
$-0.10731400$

0.43398600

0.61802800

0.02640200

0.11161100

$-0.57482800$

$-0.37364400$

$-1.09498900$

$-0.60918600$

$-0.98179800$

$-0.28960600$

$-1.56545400$

$-1.36599300$

2.07655500

2.40355600

2.40588900

2.52235100

0.57660200

0.38432100

1.11331600

1.23119100

0.26940800

$-2.04923000$

0.40463500

0.11851300

$-0.30675900$

$-0.69802000$

$-0.31198800$

0.70754000

$-0.70778300$

$-0.92116600$

1.26954200

2.05989800

0.96648400

0.95237000

$-0.92173600$

$-1.35359200$

$-1.62506700$

$-0.73773300$

$-0.46889700$

$-0.80615500$

$-0.27497300$

$-0.39969400$

$-0.61149000$ 
$\mathrm{H}$

C

$\mathrm{H}$

$\mathrm{H}$

$\mathrm{H}$

\section{p-TS1-cf2}

C

C

C

C

C

C

$\mathrm{H}$

$\mathrm{H}$

$\mathrm{H}$

$\mathrm{H}$

C

$\mathrm{H}$

$\mathrm{H}$

C

$\mathrm{H}$

$\mathrm{H}$

C

O

$\mathrm{N}$

C

C

C

C

C

$\mathrm{H}$

C

$\mathrm{H}$

$\mathrm{H}$

$\mathrm{H}$

C

$\mathrm{H}$

$\mathrm{H}$

$\mathrm{H}$

C

O

o

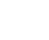

(

(

(

(1)

(

6.43851500

7.51031600

6.21747200

8.52785100

8.42600300
$-0.90988000$

2. 24805500

3. 95190400

0.37278600

2.81359500
$-1.01884000$

$-0.34580800$

$-0.06470100$

$-0.67119100$

$-0.19523400$
$-0.69289300$

$-1.64914300$

$-3.00246500$

$-3.45055700$

$-2.50430300$

$-1.14808300$

0.50741600

$-3.73112800$

$-2.84330200$

$-0.43159600$

$-4.92563900$

$-5.52586900$

$-5.17343100$

$-5.32116500$

$-6.39376100$

$-4.77587300$

$-5.04024400$

$-5.43405300$

$-4.22624900$

$-4.01850900$

$-2.74066700$

$-5.13011300$

$-2.62126400$

$-4.98885800$

$-6.11565600$

$-3.73132400$

$-1.63663900$

$-5.86838000$

$-3.61656600$

$-3.71975300$

$-4.54928200$

$-2.99033600$

$-3.24604000$

$-1.48225800$

$-1.38895400$

$-0.51357900$
$-2.25596100$

$-2.70028400$

$-2.74820000$

$-2.32147100$

$-1.96669000$

$-1.94132700$

$-2.93288400$

$-3.04265400$

$-1.67045200$

$-1.63349800$

$-2.10434700$

$-2.78974400$

$-2.27134700$

$-0.64956600$

$-0.50934500$

0.05507000

$-0.42659700$

$-1.23832400$

0.62752900

1.79522200

2. 38070900

2.45381800

3. 61206100

3.64590900

2.02949000

4.23812100

4.05653300

4.12835900

5.18053100

0.57345800

0.39011300

$-0.23470700$

1. 52526800

1.69992600

0.45985800

2.51593200
$-0.44292300$

0.49615300

0.18132300

$-1.07976700$

$-2.05360100$

$-1.74318100$

$-0.30940700$

0.92974600

$-3.04457100$

$-2.50170600$

$-1.32843500$

$-0.72357000$

$-2.38303600$

$-0.95545400$

$-1.13587100$

$-1.58742100$

0.53090400

1.36163400

0.91373100

0.13925700

$-0.02006000$

$-0.40670500$

$-0.68460000$

$-1.11306600$

$-0.24275400$

$-1.24339000$

$-0.77769100$

$-1.53006200$

$-1.76984300$

2.29367300

2. 98015600

2.39129800

2.53757400

0.39180700

0.34651900

0.73507100 
$\mathrm{H}$

$\mathrm{Pd}$

C

$\mathrm{N}$

C

O

C

$\mathrm{H}$

$\mathrm{H}$

$\mathrm{H}$

C

O

O

$\mathrm{H}$

$\mathrm{H}$

C

$\mathrm{H}$

$\mathrm{H}$

C

C

C

C

$\mathrm{H}$

C

$\mathrm{H}$

C

$\mathrm{H}$

$\mathrm{H}$

$\mathrm{H}$
0.35263300

0.46256200

3. 35515400

2. 28542000

2.50368100

1. 56255600

3.88945500

4. 61626200

4.23418200

3.84803900

2. 80312600

3.53534200

1. 50286200

4.15330400

$-1.32285500$

3. 95130600

3.20042600

4.07097100

5.27404400

6.47207900

5.33623600

7.70434100

6.43985100

6.56559000

4.41555200

7.75344600

8.62325900

6.59517200

8.71086300
2.02539900

$-0.64544300$

$-0.48342600$

$-1.46734500$

$-2.75285900$

$-3.57420100$

$-3.34882000$

$-2.84736900$

$-3.23960500$

$-4.40782400$

0.77116600

1.54159800

0.96187400

$-0.89548400$

$-2.98207500$

$-0.03825700$

0.58151200

$-0.93863500$

0.68402600

$-0.04238900$

2.07596800

0.60201200

$-1.12463500$

2. 72504100

2.65033900

1. 99075500

0.02188700

3.80565500

2. 49732500
0.94647100

0.31899700

0.52926300

0.31908700

0.13553200

$-0.15523200$

0.28762300

$-0.35783200$

1.32197100

0.03284000

1.24177100

1.82792200

1.07195900

1.15363600

1.49505900

$-0.84506900$

$-1.35003500$

$-1.45819300$

$-0.73852000$

$-0.76022400$

$-0.59806100$

$-0.64443900$

$-0.87601600$

$-0.48425500$

$-0.56036000$

$-0.50693300$

$-0.66668500$

$-0.37339200$

$-0.41861100$

\section{p-TS1-cf3}

C

C

C

C

C

C

$\mathrm{H}$

$\mathrm{H}$

$\mathrm{H}$

$\mathrm{H}$

C

$\mathrm{H}$
0.65711400

0.88010400

2.16466000

3.27105400

3.05836800

1.77207000

$-0.47193900$

0.03314300

2.32587000

3.91454200

4.66843200

5.41024400
$-2.30501600$

$-2.74223000$

$-3.00764400$

$-2.83680900$

$-2.48492300$

$-2.22404900$

$-2.82900900$

$-2.85603600$

$-3.31321500$

$-2.35184000$

$-2.86395100$

$-2.99318600$
$-0.38176200$

0.94465500

1. 40435500

0.55605300

$-0.78489100$

$-1.24422000$

$-0.93136800$

1.61776400

2.43666800

$-1.44002900$

1.12556500

0.33277100 
4.78850800

4.94547900

5.97122500

4.26830900

4.86519000

5.58789600

3.97801000

4.02235700

2.87598200

5.26244600

3. 01265600

5.38172700

6.13709400

4.24947600

2.12629100

6.35790900

4.33027800

3. 05186000

2.34044900

3. 60156700

2. 49231300

1.52025300

1. 35459000

0.55065000

$-0.49902800$

$-3.38533500$

$-2.30325600$

$-2.48977300$

$-1.53076300$

$-3.86377500$

$-4.17716100$

$-3.81412700$

$-4.61574200$

$-2.82115600$

$-3.47856600$

$-1.58877100$

$-4.18222500$

$-3.96969000$

$-3.16656500$

$-4.21634200$

$-5.18736000$

$-5.05645200$

$-6.47216600$

$-6.18301300$
$-3.68230200$

$-1.52021800$

1.84518900

1.84346400

$-1.51827000$

2.23159000

$-1.41438200$

2. 69222000

$-0.37016000$

0.83600800

$-0.39935900$

$-0.15348900$

0.66687700

1.07517900

1.81456700

2.38602300

2.44422900

3.58484600

3. 60770500

2.00441500

4.18938000

4.02190600

4.06926200

5.10480900

0.66553300

$-0.16017100$

0.61598200

1.60060900

1.76993700

0.55560300

2.60683900

$-0.52057800$

$-0.32384400$

$-1.30355400$

$-2.53508500$

$-3.37648800$

$-3.02400600$

$-2.51809500$

$-4.09739200$

$-2.81802600$

1.09407000

1.98458000

1.24071000

$-0.48313200$

$-0.41756900$

$-0.15268600$

$-1.46963200$

0.44842000

1.78766800

$-0.06746800$

2. 59120900
0.22427600

$-0.37795400$

0.04476900

$-1.10723700$

$-0.70595800$

0.50757600

$-1.28144800$

$-1.55054100$

$-0.82567900$

$-1.85961600$

2.21611100

2.14904200

3. 16225700

2.20966200

$-0.35153400$

$-0.11035400$

$-0.63222600$

$-0.41565900$

$-0.65532300$

$-0.81957800$

$-1.24924500$

$-1.35918400$

$-1.66646300$

$-2.58670400$

$-1.84999700$

$-0.89938500$

$-0.88946000$

$-1.38370500$

$-0.41309700$

$-1.38626300$

0.78409800

1.48166500

0.97100600

1.00355300

1.39287700

0.79120400

1.56678100 
$\mathrm{H}$

C

$\mathrm{H}$

C

$\mathrm{H}$

$\mathrm{H}$

$\mathrm{H}$

$\mathrm{H}$

$\mathrm{H}$
$-4.06437900$

$-7.60244900$

$-6.58820300$

$-7.45960500$

$-6.06353200$

$-8.59212900$

$-8.33744900$

$-0.37401600$

1. 62705900
2.20311100

0.73274100

$-1.10804100$

2.06611500

3. 62887100

0.31498400

2.69195600

2.17150800

$-1.91056500$
1.54960900

0.96410300

0.49255300

1. 35345600

1.86646100

0.79842300

1.49029000

$-0.61733800$

$-2.27529100$

\section{p-TS1-cf4}

C

C

C

C

C

C

$\mathrm{H}$

$\mathrm{H}$

$\mathrm{H}$

$\mathrm{H}$

C

$\mathrm{H}$

$\mathrm{H}$

C

$\mathrm{H}$

$\mathrm{H}$

C

O

$\mathrm{N}$

C

C

C

C

C

$\mathrm{H}$

C

$\mathrm{H}$

$\mathrm{H}$

$\mathrm{H}$

C

$\mathrm{H}$

$\mathrm{H}$
$-0.73899100$

$-1.43505500$

$-2.81517200$

$-3.53933500$

$-2.84772700$

$-1.47504500$

0.46439600

$-0.89247200$

$-3.34899700$

$-3.40325700$

$-5.04884900$

$-5.46116300$

$-5.43435800$

$-5.54047700$

$-5.24419100$

$-6.63579600$

$-5.04412500$

$-5.34289100$

$-4.27972400$

$-3.95831200$

$-2.65326600$

$-5.00311600$

$-2.44699300$

$-4.77394100$

$-6.00247900$

$-3.49114400$

$-1.44437100$

$-5.60440300$

$-3.30723900$

$-3.82054000$

$-3.30276900$

$-4.66972300$
$-2.32782400$

$-2.06695700$

$-2.23015300$

$-2.68467500$

$-3.03884200$

$-2.84830800$

$-2.94701100$

$-1.68850200$

$-1.95676800$

$-3.42725300$

$-2.65665000$

$-3.58572400$

$-2.53412800$

$-1.46831500$

$-1.62212500$

$-1.42510700$

$-0.14997500$

0.12390200

0.69633300

1.99683800

2.53636500

2. 80421500

3.87753100

4.11365800

2.38931100

4.66111800

4.28352200

4. 71330900

5.68759400

0.32278700

1.17594200

0.08756400
$-0.33519700$

$-1.53482700$

$-1.61503100$

$-0.50469500$

0.66611500

0.75489400

$-0.38975300$

$-2.39780100$

$-2.52098600$

1. 51791200

$-0.50384800$

$-0.09183700$

$-1.51961800$

0.35636500

1.39484400

0.32557100

$-0.24277900$

$-1.39888100$

0.54599300

0.05092200

0.10401100

$-0.42032500$

$-0.27396900$

$-0.82769600$

$-0.45917800$

$-0.74656100$

$-0.20969200$

$-1.18970500$

$-1.04883800$

1.89142200

2.33044400

2.54103000 
$\mathrm{H}$

C

O

$\mathrm{O}$

$\mathrm{Pd}$

$\mathrm{H}$

C

$\mathrm{N}$

C

O

C

$\mathrm{H}$

$\mathrm{H}$

$\mathrm{H}$

C

O

O

$\mathrm{H}$

C

$\mathrm{H}$

$\mathrm{H}$

C

C

C

C

$\mathrm{H}$

C

$\mathrm{H}$

C

$\mathrm{H}$

$\mathrm{H}$

$\mathrm{H}$

$\mathrm{H}$

$$
\begin{array}{r}
-3.12939700 \\
-1.43858300 \\
-1.36916900 \\
-0.46774100 \\
0.47280100 \\
0.40377400 \\
3.37611900 \\
2.28699900 \\
2.49145600 \\
1.54457800 \\
3.87345000 \\
4.21449700 \\
3.82254200 \\
4.60780100 \\
2.83191000 \\
3.52652400 \\
1.57699100 \\
4.16633000 \\
3.97377300 \\
3.20485000 \\
4.13998200 \\
5.26158400 \\
5.25526700 \\
6.49231800 \\
6.44996900 \\
4.30757600 \\
7.69012900 \\
6.51260100 \\
7.67115400 \\
6.42641500 \\
8.63579800 \\
8.60187500 \\
-0.95266100
\end{array}
$$

$-0.52212100$

1. 74823000

0.52264000

2.47203000

$-0.66235900$

1.95600000

$-0.56866500$

$-1.52807700$

$-2.81052400$

$-3.61249900$

$-3.42348600$

$-3.36047800$

$-4.47076600$

$-2.90375500$

0.66858500

1. 34941200

0.95350800

$-1.00796600$

$-0.09320300$

0.50110900

$-0.98170600$

0.68354000

2. 07351100

0.01414700

2.77708900

2.60407900

0.71331000

$-1.06581500$

2.09951800

3. 85522300

0.17766800

2.64802900

$-3.09852500$
1.85543500

0.45332200

0.23200800

0.95956000

0.21970000

1.06019500

0.46234900

0.22985900

0.00061700

$-0.31189100$

0.13161800

1.17106300

$-0.16628300$

$-0.49004100$

1.20496500

1.92932900

0.88133800

1.07754600

$-0.89870300$

$-1.40606700$

$-1.51816400$

$-0.75841000$

$-0.58720000$

$-0.77112800$

$-0.43479200$

$-0.56086200$

$-0.61761200$

$-0.90897900$

$-0.44956800$

$-0.30096800$

$-0.63369400$

$-0.33163700$

1.67562500

\section{$o_{1}$-TS1-cf2}

$\mathrm{C}$
$\mathrm{C}$
$\mathrm{C}$
$\mathrm{C}$
$\mathrm{C}$
$\mathrm{C}$
$\mathrm{H}$
$\mathrm{H}$

$-3.43522200$

3.53798100

$-0.86142700$

3.17028000

$-1.14472900$

2.58204500

$-0.16254500$

2.36347900

1.13602500

2.79971300

1.42722300

3. 36765300

0.43843100

3.98467400

$-1.62777100$

3.15715600

$-0.12358400$ 
$-3.50592300$

$-4.93082700$

$-0.98827400$

$-0.88611600$

0.01655900

$-1.59753900$

$-2.56271700$

$-0.93255900$

$-1.65790400$

$-0.61257800$

$-2.89842300$

$-3.03415500$

$-2.32702500$

$-3.92328400$

$-2.51548800$

$-4.13119900$

$-4.44536200$

$-3.42427900$

$-1.93821400$

$-4.83114800$

$-3.56958500$

$-4.15305300$

$-4.42537200$

$-4.08051400$

$-4.94961900$

$-1.34565400$

$-1.57729600$

$-0.27937100$

0.08628500

0.45231900

2.88920100

1.83454400

1.96433700

0.97008000

3.33182200

3. 21482200

3.78939300

4.00474300

2.66249700

3.56247800

1. 38121700

3.88197100

2.82211400

1.78383500
2.69568900

3.69115100

1.69273300

2.36424100

1.49131000

0.36493700

0.55559900

$-0.03406800$

$-0.73052400$

$-1.22916700$

$-1.14994200$

$-2.31493900$

$-2.51310900$

$-3.31037400$

$-3.69774300$

$-4.46529000$

$-3.18005600$

$-4.66365700$

$-3.84376200$

$-5.21633600$

$-5.56627300$

$-0.51775700$

$-0.78296500$

0.56466200

$-0.85034100$

$-1.52205000$

$-0.29651900$

$-2.08642400$

1.09903300

$-1.40802900$

0.91900000

1.93855800

3. 17575000

3. 95814200

3.71180300

4.73188900

3.09621100

3.71184700

$-0.06377300$

$-0.69275300$

$-0.19264700$

1. 35820500

0.15352000

$-0.16414100$
2.43863500

0.68662500

2.21008800

3.07277600

1.83022100

2.72633600

3.19993000

3.49988800

1.66009500

1.24917500

1.21489100

0.39540800

$-0.80529000$

0.82472600

$-1.53202500$

0.07475000

1.76757300

$-1.11211000$

$-2.43856200$

0.43003600

$-1.69772500$

1.64710500

2.67644400

1.55328700

0.98104000

$-1.32407300$

$-1.29512200$

$-1.82874700$

$-1.00792300$

$-2.04140800$

$-0.45115800$

$-0.47830700$

$-0.02980900$

0.08069300

0.36121400

0.72762400

1.14233300

$-0.50294500$

$-1.61296800$

$-2.12400300$

$-1.95602700$

$-0.58124800$

0.90899700

1.05945700 
$\mathrm{H}$

C

C

C

C

$\mathrm{H}$

C

$\mathrm{H}$

C

$\mathrm{H}$

$\mathrm{H}$

$\mathrm{H}$

$\mathrm{H}$

\section{$o_{1}$-TS1-cf3}

3.05238700

3.73830600

3.25385700

5.07913800

4.09462000

2. 21351600

5.92313900

5.46387300

5.43153500

3.70579900

6.96207800

6.08696400

$-1.71128900$
0.88129500

$-1.04064900$

$-2.31830200$

$-0.89785400$

$-3.42933600$

$-2.43396100$

$-2.00718300$

0.09053800

$-3.27692000$

$-4.41424900$

$-1.88141500$

$-4.14253800$

3.36618000
1.69813900

1.00440700

0.69444400

1. 38013000

0.75766600

0.39822200

1.44391100

1.62573900

1.13247800

0.51286700

1.73805800

1.18229800

$-2.13113900$
C

C

C

C

C

C

$\mathrm{H}$

C

$\mathrm{H}$

$\mathrm{H}$

C

$\mathrm{H}$

$\mathrm{H}$

C

$\mathrm{O}$

$\mathrm{N}$

C

C

C

C

C

$\mathrm{H}$

C

$\mathrm{H}$

$\mathrm{H}$

$\mathrm{H}$

C

H
$-3.49487300$

$-2.13745200$

$-1.60057800$

$-2.46831000$

$-3.83021600$

$-4.34262500$

$-0.45156500$

$-1.97016300$

$-2.45168500$

$-0.89204200$

$-2.27528100$

$-3.08679400$

$-2.61718100$

$-1.03998600$

0.09151400

$-1.22858100$

$-2.48036400$

$-2.82318000$

$-3.34353800$

$-4.01574300$

$-4.54164600$

$-3.06804800$

$-4.87595000$

$-4.27136600$

$-5.21292700$

$-5.80718100$

$-0.01508000$

0.61922800
3.61287900

3.31708800

2.53345500

2.06994900

2.38807100

3. 13684400

3.03252300

1. 24524200

1.61450900

1.37307400

$-0.26976800$

$-0.43654900$

$-0.67376700$

$-1.08575100$

$-0.73683800$

$-2.27696500$

$-2.69906400$

$-2.28819200$

$-3.53746600$

$-2.70462300$

$-3.95545800$

$-3.85383100$

$-3.54209700$

$-2.37568100$

$-4.60574400$

$-3.86975100$

$-3.04338200$

$-2.47435400$
$-1.02827600$

$-0.96213600$

0.08700000

1.10837500

1.03719800

$-0.02255400$

0.50046400

2.27717400

3.18985700

2. 40570800

2.13587100

1. 42420600

3.09825700

1.76141300

2.08715900

1.09126400

0.53187200

$-0.76899700$

1.23678800

$-1.35515600$

0.65135700

2.23829800

$-0.63970100$

$-2.35784700$

1.20469600

$-1.09223600$

0.76039900

0.07394500 


\section{$o_{1}$-TS1-cf4}

0.55269100
-0.31426800
-1.84667000
-1.94602000
-0.89015800
-0.07839500
-0.22145900
2.67236900
1.54744500
1.65046100
0.63077900
2.98882700
3.84030700
2.95823200
3.12024900
2.27757600
3.09730100
0.97919500
3.53668800
3.06766900
2.18670200
3.28387700
4.25828300
4.08802000
5.56128200
5.18989200
3.08178800
6.66748700
5.70881700
6.48391800
5.03826700
7.67119600
-4.34343700
-5.49995900
-3.89137700
-1.46409700

$-3.24769800$

1.67150000

$-3.97926400$

0.28717500

$-1.34532200$

$-1.39690800$

$-0.13320900$

$-1.14738700$

$-1.91653900$

$-2.07740600$

$-1.29861100$

1.09760600

$-2.17224800$

$-0.70906700$

1.00716200

$-0.52274700$

1.90822900

$-0.22217700$

3.01352800

0.49218000

3. 67875000

0.87840700

3.58979900

0.91221100

3.16290200

0.38101900

4.66991400

0.74933200

3.42078500

1.98621900

0.01567700

$-1.63424700$

$-0.50304800$

$-2.36034100$

$-0.27198800$

$-1.65373200$

1. 56673800

$-0.89261900$

0.20430600

0.75393200

$-0.35490300$

1.08330100

0.93555500

1.54134000

$-0.70302300$

0.56158300

$-2.06165200$

0.26998100

$-0.19744600$

0.65772400

$-2.89478600$

0.07664200

$-2.46472500$

0.18825200

$-1.02503100$

0.46365200

0.85568400

0.89109800

$-2.37866000$

0.17227600

$-3.94666900$

$-0.15130000$

$-0.61528700$

0.54338500

$-3.02649900$

0.02163900

2.04424900

1. 82331700

3. 36542800

$-0.05503300$

4.21467100

$-1.84159800$

3.70788400

$\begin{array}{ll}\text { C } & -2.30002400 \\ C & -1.09033900 \\ C & -0.97636200 \\ C & -2.12469000\end{array}$

3.47480400

2.98033600

2.42287200

2.38906000
$-2.11807300$

$-1.64252300$

$-0.34666200$

0.49074600 
$-3.33121700$

$-3.42084200$

$-2.37278400$

0.14102300

$-4.22797700$

$-2.00939700$

$-1.71490400$

$-1.18084300$

$-3.28235100$

$-4.05489600$

$-3.02695700$

$-3.92647900$

$-4.92627300$

$-3.32651000$

$-3.76728300$

$-2.92728000$

$-5.02626700$

$-3.33930400$

$-5.44586200$

$-5.66604900$

$-4.59755100$

$-2.66967500$

$-6.43163300$

$-4.91662900$

$-2.14984700$

$-1.22078900$

$-2.26567400$

$-2.06867800$

$-1.63130200$

$-1.51464100$

$-0.68690200$

0.25915300

3.15983100

2.07106800

2.24485300

1. 26650100

3.61760500

4.41484900

3.57251600

3.85048400

2.60675600

3.25464600

1.39395700

3.96363100
2.90189300

3. 42507700

3.89411400

2.88558400

2.85445000

1.84456100

2.66911700

1.13208300

1.20138700

1.95791500

0.79995400

0.14266300

0.40179600

$-1.10445100$

$-2.00684700$

$-2.36308800$

$-2.59970500$

$-3.34837200$

$-3.54457800$

$-2.30956900$

$-3.93125200$

$-3.62939600$

$-3.99128600$

$-4.67987600$

$-1.48948500$

$-1.06475500$

$-1.18170200$

$-2.57856900$

$-1.67483600$

$-0.47191300$

$-2.40847000$

0.67200600

0.56787200

1.53946200

2.77550300

3.53163600

3. 40426800

2. 83854300

4.41019800

3.50788900

$-0.66648500$

$-1.28706300$

$-1.01392100$

0.98498700
0.00626500

$-1.28614100$

$-3.11781700$

0.17948000

0.61265100

1.90434700

2. 57022100

1.93787000

2. 50784600

2. 66453700

3.49463500

1. 61431300

0.95631000

1.55097200

0.52952600

$-0.54616200$

0.63644800

$-1.45613400$

$-0.29896800$

1.46206400

$-1.34010500$

$-2.26203500$

$-0.20483100$

$-2.05901200$

2. 33314900

1.93835300

3.37519700

2.31706600

$-0.76899800$

$-0.45832800$

$-1.29985500$

$-0.41410500$

$-0.49310600$

$-0.30005900$

0.13448000

0.45165600

0.27711600

$-0.20663100$

$-0.14784000$

1.34216800

$-1.23675700$

$-2.05062500$

$-0.80933000$

$-1.10654600$ 


$$
3.72122700
$$

2.88539200

4.03521300

4.87331800

4.64392300

6.19336400

5.70846400

3.62250300

7.26187100

6.38511400

7. 02102900

5.51280400

8.28042400

7.85105900

0.21460300

$-4.37786100$

$-0.20057800$
0.10368300

0.88317100

$-0.33888400$

1. 43699100

0.99517100

1.43710600

$-0.86722700$

0.77951500

$-2.24706300$

0.70336100

$-0.40133300$

0.73317100

$-3.14013500$

0.58487200

$-2.61870400$

0.72676500

$-1.29115700$

0.61430100

0.66836700

0.79605700

$-2.66473500$

0.54063200

$-4.20737400$

0.52480400

$-0.91302300$

0.58286100

$-3.36031300$

$-1.92402900$

0.44989000

3. 79831100

3.04084600

$-1.30120300$

$-1.64172400$

$-2.26502400$

\section{$o_{1}$-TS1-cf5}

C

C

C

C

C

C

$\mathrm{H}$

$\mathrm{H}$

$\mathrm{H}$

$\mathrm{H}$

C

$\mathrm{H}$

$\mathrm{H}$

C

$\mathrm{H}$

$\mathrm{H}$

C

$\mathrm{O}$

$\mathrm{N}$

C

C

C

C

C
1.33616100

2.60338300

2.90886300

1.97525000

0.69810300

0.39730900

1.08940700

3.35475800

3.89390300

$-0.28251100$

2. 31726900

1. 50207600

3.22127200

2. 52732700

1.58634900

2.78407200

3. 62156300

4.43050600

3.63013200

4.18069300

3. 32935400

5.52390700

3.81660400

6.01888500
$-2.95088100$

$-3.30761000$

$-3.20865200$

$-2.71866000$

$-2.30680800$

$-2.46124400$

$-3.05670900$

$-3.68045400$

$-3.49631400$

$-2.76489100$

$-2.74509800$

$-3.24917400$

$-3.34487600$

$-1.37820800$

$-0.81660300$

$-1.52724600$

$-0.56528800$

$-1.06786300$

0.80039400

1.66221100

2.20801100

2. 02804600

3.15040700

2.93469400
2.95297500

2. 48225800

1.12477100

0.20558000

0.67533200

2. 05111700

4.00604100

3.17396800

0.77126000

$-0.14076300$

$-1.27396300$

$-1.80927300$

$-1.41397300$

$-1.94328600$

$-1.93039800$

$-3.00151200$

$-1.25833500$

$-0.49386400$

$-1.52441000$

$-0.51808700$

0.46312300

$-0.54084600$

1. 37256000

0.39939300 
$\mathrm{H}$

C

$\mathrm{H}$

$\mathrm{H}$

$\mathrm{H}$

C

$\mathrm{H}$

$\mathrm{H}$

$\mathrm{H}$

C

O

$\mathrm{O}$

$\mathrm{Pd}$

$\mathrm{H}$

C

$\mathrm{N}$

C

O

C

$\mathrm{H}$

$\mathrm{H}$

$\mathrm{H}$

C

O

O

$\mathrm{H}$

C

$\mathrm{H}$

$\mathrm{H}$

C

C

C

C

$\mathrm{H}$

C

$\mathrm{H}$

C

$\mathrm{H}$

$\mathrm{H}$

$\mathrm{H}$

$\mathrm{H}$
6.16728500

5.16591700

3.14216000

7.07022200

5.54942100

2.71309200

3.09539300

2. 67979200

1.68721800

1.91155600

1.66661500

1.01693100

$-0.16497900$

0.09002900

$-2.95946800$

$-1.93489900$

$-2.12917300$

$-1.22967500$

$-3.43108600$

$-3.55185800$

$-3.41159900$

$-4.29359400$

$-2.30103200$

$-2.92298300$

$-1.05442100$

$-3.60847000$

$-3.83109600$

$-3.21515400$

$-4.04911400$

$-5.12241500$

$-5.18886300$

$-6.28296500$

$-6.38594200$

$-4.29390400$

$-7.48241000$

$-6.24905300$

$-7.53643200$

$-6.41884900$

$-8.37319500$

$-8.46893500$

$-0.59664800$
1.59717200

3. 50337100

3.58642400

3.20741300

4. 22117400

1.42436400

2.41806300

0.83983400

1.53023700

1.75602300

0.53709500

2. 71113700

$-0.44240300$

2.34192100

$-0.07244200$

$-1.12090300$

$-2.35973300$

$-3.26399800$

$-2.78219400$

$-2.27782400$

$-3.86032500$

$-2.52101800$

1. 32077300

2.28084500

1. 37731200

$-0.20186800$

$-0.10590200$

0.25953500

$-1.15860800$

0.67079600

2.02686700

0.03890800

2.73272000

2.53465900

0.74124100

$-1.01762200$

2.09286700

3. 78515500

0.23290000

2.64338700

$-2.19795400$
$-1.30055100$

1. 34801800

2.10320500

0.38145300

2.06706500

$-2.48222900$

$-2.72687400$

$-3.40439100$

$-2.10160500$

0.50554000

0.55254200

0.44511200

0.08029000

0.24883300

$-0.61575200$

$-0.55859400$

$-0.96362000$

$-0.84237800$

$-1.61608300$

$-2.58151300$

$-1.77581300$

$-0.99573400$

$-0.73770700$

$-1.14432600$

$-0.29072000$

$-1.48667700$

0.67966100

1. 51037100

0.89457700

0.57439600

0.91731200

0.10809300

0.79767900

1.26399100

$-0.01535600$

$-0.15366300$

0.33108800

1.06631600

$-0.37529200$

0.23944900

2. 40649400

\section{$o_{1}$-TS1-cf6}


C

C

C

C

C

C

$\mathrm{H}$

$\mathrm{H}$

$\mathrm{H}$

$\mathrm{H}$

C

$\mathrm{H}$

$\mathrm{H}$

C

$\mathrm{H}$

$\mathrm{H}$

C

O

$\mathrm{N}$

C

C

C

C

C

$\mathrm{H}$

C

$\mathrm{H}$

$\mathrm{H}$

$\mathrm{H}$

C

$\mathrm{H}$

$\mathrm{H}$

$\mathrm{H}$

C

O

O

$\mathrm{H}$

C

$\mathrm{N}$

C

O

C

$\mathrm{H}$

$\mathrm{H}$
2.39284000

1.21099800

1.19012300

2. 39483500

3. 57268700

3.57830100

2.39618100

0.19408900

4.49802000

4.50895900

2.42728700

1.42963500

3.07786700

2.94990500

3.64173000

3.52414300

1.83866300

0.76691600

2.11902700

3.23035100

3.13878100

4.39877900

4.21435000

5.46976300

4.46146400

5.38043000

4.13772400

6.37826000

6.21814900

1.09761900

0.92828600

0.14846000

1. 46075600

1.84454400

1.76596500

0.86269500

$-0.06784000$

$-2.68018900$

$-1.63865100$

$-1.65827100$

$-0.72700400$

$-2.79525600$

$-3.77455000$

$-2.70800200$
3.80774300

3.28708300

2. 58724700

2.45788500

3.00175900

3. 65197400

4.33793400

2.90373000

2. 91816100

4.05854900

1. 78257500

1.78300500

2. 36908700

0.32658800

0.14861500

0.15037900

$-0.71437600$

$-0.45333100$

$-2.00213300$

$-2.32752900$

$-2.06472400$

$-2.88783100$

$-2.32532900$

$-3.17332800$

$-3.08537600$

$-2.88802900$

$-2.09916200$

$-3.60924200$

$-3.10281600$

$-3.02922700$

$-3.10123300$

$-2.77921700$

$-3.98351200$

$-1.45116100$

$-0.21688100$

$-2.30101700$

$-1.83262100$

0.32232800

1. 35974300

2.40769500

3.28025900

2. 61775200

2.48610500

1.89061700
$-2.10060500$

$-1.58450800$

$-0.35393200$

0.38588300

$-0.13911100$

$-1.37386500$

$-3.04908800$

0.44431400

0.42760300

$-1.76232900$

1.74096400

2.18828300

2.39975100

1.69657600

2. 53094700

0.78472300

1.85718000

2.39509300

1.44609900

0.60107700

$-0.77810500$

1.11711100

$-1.62371800$

0.26605200

2.18306800

$-1.09805300$

$-2.68301100$

0.67163600

$-1.75503000$

1.71926200

2.79724600

1.23784200

1.33575000

$-1.21324100$

$-1.36395000$

$-1.29250600$

$-1.22772300$

0.48569900

0.49434800

1.29645200

1.31130400

2.27996900

1.81228800

3.09555500 


\section{$o_{1}$-TS1-cf7}

$\mathrm{C}$
$\mathrm{C}$
$\mathrm{C}$
$\mathrm{C}$
$\mathrm{C}$
$\mathrm{C}$
$\mathrm{H}$
$\mathrm{H}$
$\mathrm{H}$
$\mathrm{H}$
$\mathrm{H}$
$\mathrm{C}$
$\mathrm{H}$
$\mathrm{H}$
$\mathrm{C}$
$\mathrm{H}$
$\mathrm{H}$
$\mathrm{C}$
$\mathrm{O}$
$\mathrm{N}$

C

C

C

C

C

C

$\mathrm{H}$

$\mathrm{H}$

H

$\mathrm{H}$

$\mathrm{H}$

C

$\mathrm{H}$

$\mathrm{H}$

C

$\mathrm{H}$

$\mathrm{H}$

C

O

N
$-2.71921400$

$-2.03162200$

$-2.32642300$

$-1.16663300$

$-3.12204700$

0.27976300

$-0.01736600$

$-3.78242900$

$-3.30143400$

$-4.13932200$

$-4.93574900$

$-4.90369900$

$-6.04182600$

$-5.95222500$

$-4.04609200$

$-7.09325700$

$-6.08052600$

$-7.05051800$

$-5.91036200$

$-7.94526100$

$-7.86802300$
3.62383100

$-1.02310000$

$-2.08201000$

$-0.88513900$

0.19685800

3. 42500700

0.83275500

0.67731100

0.72147600

1.68894000

$-0.29783600$

$-1.44759100$

$-0.08963200$

$-2.36697600$

$-1.62424400$

$-1.00639100$

0.80166000

$-2.14896400$

$-3.25490500$

$-0.82762300$

$-2.86457500$
2.69293600

0.10061200

0.61075600

$-0.90679600$

1. 47717100

$-2.12910400$

$-0.57687500$

$-0.55068500$

$-1.53418900$

$-0.32394600$

$-0.54997400$

$-1.34978600$

0.28381800

$-1.31655800$

$-1.99426500$

0.32008300

0.90771500

$-0.48175200$

$-1.94169000$

0.97093800

$-0.45655700$
0.70923900

2.04792100

2.64313200

1. 93846400

0.57927900

$-0.01480400$

0.23895700

2.62854300

3.67599100

$-0.19522400$

$-1.07079600$

2. 61488100

1.85818500

3. 31104400

3.40546000

2.71837600

4. 01803800

4.33382100

4.99122500

4. 36580600
3.70417900

3.97892800

3.46055900

2. 61148200

2. 32012800

2.89693400

4.13168400

4. 62225200

3. 69863300

2.50222000

2.71924500

2.12647700

2.00556000

2. 90335200

0.79203000

$-0.04987100$

0.69673600

0.78678200

1.77634100

$-0.34630900$
$-2.15514400$

$-1.85923000$

$-0.70939700$

0.15639300

$-0.13432400$

$-1.28720000$

$-3.03659800$

$-2.51591400$

$-0.48392100$

0.92166000

$-1.47652500$

1.42697200

2. 21104800

1. 75804500

1. 33331800

1. 31028000

2. 23913700

0.12756600

$-0.17606900$

$-0.67287100$ 
C
4.26664500

3.07565800

5.46606000

3.14107000

5.51287800

6.37241700

4.34186300

2.22417300

6.45763800

4.35965000

5.14701000

4.88735700

4.88762700

6.22916900

1.72407300

1.54102200

0.77141100

$-0.16364300$

$-0.28532500$

$-3.09564100$

$-2.00027100$

$-2.06663500$

$-1.08911000$

$-3.32270400$

$-3.46224600$

$-3.21595300$

$-4.21364300$

$-2.51579100$

$-3.13050900$

$-1.33418900$

$-3.74783600$

$-3.93402100$

$-3.28419000$

$-4.17678700$

$-5.20027200$

$-5.20009900$

$-6.39750300$

$-6.36894200$

$-4.27555100$

$-7.56931300$

$-6.41237600$

$-7.55716200$

$-6.35088200$

$-8.49008100$
$-1.67048000$

$-2.43048600$

$-2.25951600$

$-3.75852900$

$-3.57322900$

$-1.66292500$

$-4.33076200$

$-4.33240800$

$-3.99897500$

$-5.35682700$

$-0.23614800$

$-1.07592400$

0.70366500

$-0.23733200$

$-1.87687100$

$-0.65623900$

$-2.77730300$

$-2.36384800$

0.36540300

0.01248700

0.97310100

2.03470000

2.84422000

2.34136400

1. 59481800

3. 32592000

2. 32680300

$-1.40856200$

$-2.40269500$

$-1.42082900$

$-0.00944500$

0.39286900

0.28693600

1.45911100

$-0.41477800$

$-1.63066000$

0.03056300

$-2.38240800$

$-1.99363500$

$-0.71853200$

0.97679900

$-1.92878200$

$-3.32473400$

$-0.35600900$
$-0.12861300$

$-0.07339400$

0.29492900

0.39131500

0.75397800

0.25431600

0.79503900

0.43981100

1.08032800

1.14970900

$-1.91041300$

$-2.55884700$

$-2.39992300$

$-1.72697100$

$-0.37495700$

$-0.55732500$

$-0.35962900$

$-0.29674500$

0.06544500

0.74591700

0.92038700

1.70489000

1.83761800

2.50011300

3.29015900

2. 95575900

1.86500600

0.57471000

0.89811100

$-0.03089400$

1. 62255300

$-0.50931700$

$-1.38632100$

$-0.42245900$

$-0.66742600$

$-1.36244400$

$-0.09229200$

$-1.48057600$

$-1.80321300$

$-0.20687300$

0.44620700

$-0.90335900$

$-2.02144800$

0.24279900 


\section{$o_{1}$-TS1-cf8}

C

C

C

C

C

C

$\mathrm{H}$

$\mathrm{H}$

$\mathrm{H}$

$\mathrm{H}$

$\mathrm{H}$

C

$\mathrm{H}$

$\mathrm{H}$

C

$\mathrm{H}$

$\mathrm{H}$

C

$\mathrm{O}$

$\mathrm{N}$

C

C

C

C

C

$\mathrm{H}$

C

$\mathrm{H}$

$\mathrm{H}$

$\mathrm{H}$

C

$\mathrm{H}$

$\mathrm{H}$

$\mathrm{H}$

C

O

O

$\mathrm{H}$

$\mathrm{Pd}$

C
2.22646900

3.38997700

3. 38718600

2.23581300

1.04330600

1.06435600

2.22600200

4.30435700

4.29454700

$-0.00494600$

0.13553900

2. 28065600

1. 27503000

2. 92778700

2. 80711900

2.05701500

2. 99386600

4.11957000

5.03557300

4.21579900

3.70320800

2. 50370900

4.51894400

2.18551100

4.18474400

5.43467600

3.01577000

1.26155200

4.83516000

2.74307700

5.40572100

5.24627800

5.53108900

6.32062900

1.47315200

1.50961600

0.51128100

$-0.33716900$

$-0.22886000$

$-3.14037000$
$-3.75897900$

$-3.66488000$

$-2.98078900$

$-2.32766400$

$-2.43251600$

$-3.16264700$

$-4.31146200$

$-4.14732700$

$-2.93304200$

$-2.80527200$

$-3.28190700$

$-1.59710100$

$-1.57838000$

$-2.16103100$

$-0.13007900$

0.53447700

0.18118600

$-0.05803500$

$-0.83716100$

0.83582900

2.17553500

2. 61505600

3.11477300

3.98592600

4.46676500

2.76321100

4.90676800

4.31315200

5.16984100

5.95761400

0.69175800

1.26375400

$-0.36482500$

1. 04238000

1.69204400

0.46977100

2.28511200

1.71643200

$-0.73872000$

$-0.72004500$
$-1.81960800$

$-1.04946900$

0.16640500

0.63070700

$-0.13210400$

$-1.34781100$

$-2.75528800$

$-1.38642800$

0.75717100

0.54495300

$-1.90068400$

1. 96163300

2.39745000

2. 64102700

1.93010100

1.50655900

2.96508900

1.16424600

1.40363700

0.10419900

0.19033500

$-0.41544500$

0.83458000

$-0.37691000$

0.87448100

1.30065000

0.25299200

$-0.83773100$

1.38720100

0.27018800

$-0.74414400$

$-1.66112600$

$-0.98521800$

$-0.24953500$

$-0.96406400$

$-0.72169100$

$-1.63040900$

$-1.65142000$

$-0.40486300$

$-0.31885200$ 


$$
\begin{aligned}
& -2.00446200 \\
& -2.11345800 \\
& -1.09821300 \\
& -3.45712600 \\
& -4.29855400 \\
& -3.40411100 \\
& -3.62480900 \\
& -2.68297300 \\
& -3.41585300 \\
& -1.44802100 \\
& -3.95503200 \\
& -3.65722400 \\
& -2.83088300 \\
& -3.86723600 \\
& -4.89021300 \\
& -4.78603000 \\
& -6.16554800 \\
& -5.92866500 \\
& -3.80153400 \\
& -7.31168300 \\
& -6.26051400 \\
& -7.19522000 \\
& -5.82969900 \\
& -8.29345700 \\
& -8.08573900
\end{aligned}
$$

\section{m-TS4-cf1}

$\mathrm{Pd}$
$\mathrm{C}$
$\mathrm{C}$
$\mathrm{C}$
$\mathrm{C}$
$\mathrm{C}$
$\mathrm{C}$
$\mathrm{H}$
$\mathrm{H}$
$\mathrm{H}$
$\mathrm{H}$
$\mathrm{H}$
$\mathrm{C}$
$\mathrm{O}$
$\mathrm{O}$
$\mathrm{C}$

$\mathrm{Pd}$

C

C

H

$\mathrm{H}$

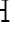

C
1.92654400

1.23316000

$-0.05386100$

$-0.39111500$

0.61578100

1.89325700

2. 20255200

1.01755900

$-0.79692900$

0.38230400

2. 64276200

3.19616400

$-0.07042900$

0.39489600

0.28492800

$-1.16148200$

$\begin{array}{rr}-1.60657500 & -0.01744100 \\ -2.74893000 & 0.64208700 \\ -3.41472300 & 1.02858500 \\ -3.36988800 & 0.97667500 \\ -2.91577400 & 0.45204000 \\ -4.43391100 & 0.73267900 \\ -3.28847000 & 2.05574500 \\ 0.37928300 & -1.29857800 \\ 0.85549000 & -2.13794000 \\ 0.80294800 & -1.04489500 \\ -1.26888400 & -0.80024500 \\ -0.03140800 & 0.97948100 \\ 0.56789600 & 1.37865600 \\ -0.81448400 & 1.71611600 \\ 0.81483600 & 0.76977100 \\ 2.17268000 & 0.44313700 \\ 0.24444600 & 0.87292200 \\ 2.94241400 & 0.22582000 \\ 2.62395800 & 0.34907500 \\ 1.00991900 & 0.65477200 \\ -0.80877500 & 1.13150400 \\ 2.36337500 & 0.33098800 \\ 3.99420600 & -0.02890500 \\ 0.55158900 & 0.74141400 \\ 2.96296300 & 0.16224000\end{array}$

$-1.60657500$

$-0.01744100$

0.64208700

1.02858500

0.97667500

0.45204000

0.73267900

$-1.29857800$

1.29857800

$-1.04489500$

$-0.80024500$

1.37865600

1.71611600

0.76977100

0.44313700

0.22582000

0.34907500

0.65477200

1.13150400

$-0.02890500$

0.16224000
$-0.64180800$

0.87156200

1.19788700

1.69391900

1.61495500

1.27062200

2.96033600

1.12532000

3.90989600

1. 37162100

3.53327500

1.78918800

2.18508100

1.95970100

0.27436100

2. 66417300

0.84938500

1.07814000

4.96626600

1.25221600

4.29595100

1. 98477800

1.88847700

2.27987000

$-1.46461500$

2.78332400

$-0.52925600$

3.50507600

$-1.68858500$

1. 58269300

$-2.34252700$ 
2.63447100

2.04264400

1.06074300

2.57960300

$-1.06194300$

$-1.13053900$

$-2.12935300$

3. 41113100

1.76995500

2.90415400

3. 63131700

3.76175400

2. 90403900

5.11553200

5.33763800

5.07088300

5.90031900

0.86453000

$-3.26494500$

$-5.79859100$

$-4.07502800$

$-3.74840800$

$-5.00381600$

$-5.33781900$

$-3.11971900$

$-5.36069900$

$-5.95286700$

$-6.78051700$

$-1.88177800$

$-1.27121600$

$-1.39840600$

$-0.42089900$

$-1.79001400$

$-2.15030500$

$-1.72618800$

$-2.84817600$

$-3.84512400$

$-2.77416100$

$-2.83777200$

$-2.21665900$

$-3.67289000$

$-3.68726400$

$-3.70601800$

$-2.79384400$
$-2.39664600$

$-2.86619800$

$-2.33063500$

$-4.17548500$

$-3.36042600$

$-2.33204600$

$-1.94678100$

$-4.55253100$

$-4.91261300$

$-4.01559400$

0.30139700

0.95666500

1. 12874200

1.62648200

1.76551800

2. 61264400

1.04489500

0.28968400

$-1.13492000$

$-0.91167700$

$-0.00789700$

$-2.14049900$

$-2.02808600$

0.08265200

$-3.00307200$

$-2.80975900$

0.94556100

$-0.81796300$

$-1.24693300$

$-0.23362100$

$-2.46333700$

$-2.44015800$

3.46067100

4.01296800

4.18566000

2.41704500

2.82769400

1.51992800

2.05483700

2.70885900

1.01655100

0.66376100

1.58308100

0.09405100
$-0.03351600$

$-1.05226900$

$-1.65133500$

$-1.60436700$

2.96940100

4.44337400

3.02244300

$-1.00706200$

$-1.62867100$

$-2.63805100$

0.24674000

$-0.84467300$

$-1.74469000$

$-1.01467400$

$-2.07448900$

$-0.53692600$

$-0.52631600$

$-1.66268400$

$-0.24687100$

0.93057600

$-0.52359200$

0.60232200

1.19736700

0.06582000

0.79391400

1.86147600

$-0.17011700$

1.38576300

$-0.79207700$

$-1.15242800$

$-0.83743600$

$-1.12620500$

0.79932000

1.67773200

$-0.01944800$

0.42746200

0.63619400

1.04903700

$-1.05870300$

$-1.88518300$

$-1.44185000$

$-2.86456500$

$-3.45088100$

$-3.14609100$ 


\section{m-TS4-cf2}

$\mathrm{Pd}$

C

C

C

C

C

C

$\mathrm{H}$

$\mathrm{H}$

$\mathrm{H}$

$\mathrm{H}$

C

O

$\mathrm{O}$

C

O

C

O

C

$\mathrm{H}$

$\mathrm{H}$

$\mathrm{H}$

$\mathrm{H}$

$\mathrm{H}$

$\mathrm{H}$

O

C

O

C

$\mathrm{H}$

$\mathrm{H}$

$\mathrm{H}$

Ag

C

C

C

C

C

C

$\mathrm{H}$
$-2.45975100$

0.74719400

1. 68646600

1.33038500

0.00334700

$-0.96815300$

$-0.56651800$

2. 71011600

$-0.28298600$

$-2.18500900$

$-1.30508600$

$-3.87930400$

$-3.25326900$

$-3.62808200$

$-5.00578300$

$-3.75061400$

$-3.69527800$

$-2.76854700$

$-4.90941600$

$-5.22234900$

$-5.89995100$

$-4.75414000$

$-5.33785100$

$-5.66824800$

$-4.63922700$

$-1.59207000$

$-0.73003400$

$-0.20087800$

$-0.32013100$

$-0.35852800$

0.71758000

$-0.96189100$

$-0.70705000$

3.84793400

6.56191800

4.31686300

4.75100600

6.09810600

5.67705400

4.38210400
0.27759600

0.54129600

2.58837200

2.56789300

2.17224800

1.80308100

1.81361100

2.20310500

2. 87038100

1.47264100

2.39667000

2. 22761900

2.55445500

3.15297100

1. 36782200

3.28907500

$-1.33678600$

$-1.98588800$

$-1.96001900$

$-2.85376700$

2.83657600

3.21626400

4.34666200

$-3.24074500$

$-2.23284200$

$-3.67091600$

$-0.63110400$

$-1.58638800$

$-2.08305700$

$-2.10842800$

$-3.20178100$

$-1.80613300$

$-1.71493300$

$-1.33747300$

$-0.07753900$

0.25903300

$-0.41410000$

0.42902300

0.59452500

$-0.24195000$

0.69028700
$-1.01334800$

0.01901700

1.56148500

0.54131800

$-0.75822300$

$-0.19024400$

2. 55511900

0.76058900

$-1.55511500$

$-0.40651200$

0.52502300

$-0.79386500$

$-1.09581800$

0.23689400

$-0.86214700$

$-1.71621000$

$-1.15628400$

$-2.06493600$

$-0.46600200$

$-1.20388800$

$-0.22881200$

$-1.64705200$

$-1.82836000$

2.14064100

2. 11425100

1.09825400

3.47911100

3.46915600

3.64942700

4.26918300

$-1.06196700$

$-1.50942200$

$-0.88173500$

$-0.21502600$

$-2.46015400$

$-2.15517600$

0.07109500

$-3.44533800$
1. 31798800 


$\begin{array}{lrrr}\mathrm{H} & 6.78032600 & 0.98129000 & -2.90593500 \\ \mathrm{H} & 6.02654400 & -0.50633600 & 1.06432700 \\ \mathrm{H} & 7.61184600 & 0.38041000 & -0.63073300 \\ \mathrm{C} & 2.42423100 & -0.23768300 & -1.89007200 \\ \mathrm{O} & 1.63233200 & -0.97817600 & -1.32397000 \\ \mathrm{O} & 2.06866400 & 0.50121200 & -2.95933900 \\ \mathrm{H} & 1.11891300 & 0.33700900 & -3.11321100 \\ \mathrm{C} & 2.36941400 & 2.13402800 & 2.42150600 \\ \mathrm{H} & 2.71761500 & 3.15215700 & 2.64094300 \\ \mathrm{H} & 1.92430700 & 1.73555200 & 3.33762700 \\ \mathrm{C} & 3.59456200 & 1.26832500 & 2.06320200 \\ \mathrm{H} & 4.33323300 & 1.34500400 & 2.87030600 \\ \mathrm{H} & 4.07130100 & 1.63730100 & 1.15342900 \\ \mathrm{C} & 3.19321400 & -0.20238700 & 1.96845900 \\ \mathrm{O} & 2.61314600 & -0.73719400 & 2.90760000 \\ \mathrm{~N} & 3.46257500 & -0.90309700 & 0.81231000 \\ \mathrm{C} & 3.05355500 & -2.31991000 & 0.78213900 \\ \mathrm{H} & 3.63622500 & -2.88699400 & 1.51706900 \\ \mathrm{H} & 1.99045200 & -2.39949400 & 1.01160400 \\ \mathrm{H} & 3.23979300 & -2.71210600 & -0.21736700 \\ \mathrm{H} & 1.04750800 & 2.90223800 & -2.00923500\end{array}$

\section{m-TS4-cf3}

$\mathrm{Pd}$
$\mathrm{C}$
$\mathrm{C}$
$\mathrm{C}$
$\mathrm{C}$
$\mathrm{C}$
$\mathrm{C}$
$\mathrm{H}$
$\mathrm{H}$
$\mathrm{H}$
$\mathrm{C}$
$\mathrm{O}$
$\mathrm{O}$
$\mathrm{C}$
$\mathrm{O}$
$\mathrm{C}$
$\mathrm{O}$
$\mathrm{C}$
$\mathrm{H}$
$\mathrm{H}$

2.54771200

0.79236500

$-0.47844300$

$-0.75248700$

0.27312900

1. 56663500

1.80884000

$-1.27616500$

0.08727400

2.47580600

3. 72141800

3.22531000

3.56450500

4.58457300

2.67482700

3.54197200

3.56230000

4.71459300

4.68663000

5.57680600
0.46029400

4.26793400

4.02751400

2.87804900

1.94254800

2.14532400

3.34027900

4.74599100

1.04425300

1.89338700

0.49560000

1.65173300

$-0.21666900$

$-0.07402600$

$-2.86203500$

$-2.28258700$

$-1.05404100$

$-3.11220400$

$-1.15427100$

0.38729200
$-0.29557800$

$-0.32717100$

0.20132500

0.95540900

1.12030200

0.58589900

$-0.12967200$

0.03349200

1.70765600

1.53696800

2. 39527300

2. 56424100

1.35702900

3. 49881600

$-0.28714600$

$-0.99493100$

$-1.33276800$

$-1.49445800$

3. 38508400

3.43693800 


$$
4.15755500
$$

5.54362700

4.44268000

5.05138400

$-0.05885500$

0.43096800

1. 57540800

$-0.41284400$

0.16709600

$-0.82999500$

$-1.24806100$

0.72967700

$-3.88912600$

$-6.44301000$

$-4.79293600$

$-4.25845600$

$-5.53449000$

$-6.06689600$

$-3.53198900$

$-5.81762700$

$-6.74971800$

$-7.43761900$

$-2.51183800$

$-1.55176200$

$-2.44090100$

$-1.46920800$

$-2.10283100$

$-1.95050500$

$-2.71849100$

$-2.88684000$

$-3.71928900$

$-2.24058400$

$-3.42388400$

$-3.00305600$

$-4.44825400$

$-4.95713500$

$-4.86034100$

$-4.38104500$

$-6.00796000$

2. 79706100

0.97901300
0.17747900

$-2.99625400$

$-4.16849700$

$-2.75153400$

$-0.40794700$

0.65027900

1.11290200

1.44206900

2.23910600

0.77616400

1.87811200

$-1.87815600$

$-1.50627100$

$-1.60006300$

$-0.44629900$

$-2.57448000$

$-2.63372400$

$-0.50865200$

$-3.35871900$

$-3.47716200$

0.31987500

$-1.63318700$

$-1.49884700$

$-1.88964600$

$-1.04856400$

$-0.80983700$

2.69903300

2.63469000

3.58623500

1. 44936000

1.27104500

0.56830500

1. 56221000

2.40875000

0.70336900

0.74759200

1.76672100

0.06982000

0.44601400

3.52488800

5.17663100
4.47260100

$-0.78623700$

$-1.54487200$

$-2.46916900$

$-1.78752200$

$-2.28717800$

$-1.99751700$

$-3.26172500$

$-3.72858100$

$-4.02324900$

$-2.69817700$

0.02501400

0.41688900

1.55459300

0.19797200

1.24359000

1.79776000

0.77226700

1. 43156200

2. 42032200

0.60872400

1.99019900

$-0.15926000$

0.52181200

$-1.38575800$

$-1.66068400$

1.62198700

2.70762300

1. 44213100

1.19432700

1.88486500

1.27492500

$-0.23298200$

$-1.01677300$

$-0.58040200$

$-1.95385200$

$-2.32797300$

$-2.59321500$

$-1.95708400$

$-0.54246100$

$-0.89349300$

\section{$m$-TS4-cf4}




\begin{tabular}{|c|c|c|c|}
\hline $\mathrm{Pd}$ & -2.49066500 & 0.31854800 & 0.65960500 \\
\hline $\mathrm{C}$ & -0.95053100 & 1.78186000 & 0.39082300 \\
\hline $\mathrm{C}$ & 0.09033500 & 1.85885900 & 1.33688900 \\
\hline $\mathrm{C}$ & 1.41396900 & 2.11841000 & 0.96178900 \\
\hline $\mathrm{C}$ & 1.69153500 & 2.30275200 & -0.40232800 \\
\hline $\mathrm{C}$ & 0.68447000 & 2.22265500 & -1.36485900 \\
\hline $\mathrm{C}$ & -0.62636300 & 1.96737900 & -0.97441500 \\
\hline $\mathrm{H}$ & -0.14080600 & 1.69765900 & 2.38613500 \\
\hline $\mathrm{H}$ & 2.70662300 & 2.51280600 & -0.72582400 \\
\hline $\mathrm{H}$ & 0.92931900 & 2.35452500 & -2.41516800 \\
\hline $\mathrm{C}$ & -3.89985600 & 2.56061700 & -0.38816600 \\
\hline 0 & -3.16109600 & 3.23124500 & 0.39968300 \\
\hline O & -3.73740600 & 1.32443100 & -0.64948600 \\
\hline $\mathrm{C}$ & -5.06313800 & 3.25934700 & -1.05185500 \\
\hline O & -3.12582400 & -2.05105700 & -1.36006000 \\
\hline $\mathrm{C}$ & -3.92124600 & -2.01233300 & -0.38396700 \\
\hline O & -3.84861100 & -1.25832800 & 0.64477200 \\
\hline C & -5.12843400 & -2.93643600 & -0.42674800 \\
\hline $\mathrm{H}$ & -5.32825000 & 2.75572100 & -1.98321600 \\
\hline $\mathrm{H}$ & -5.92373200 & 3.21008000 & -0.37453200 \\
\hline $\mathrm{H}$ & -4.82391000 & 4.31020500 & -1.22687600 \\
\hline $\mathrm{H}$ & -5.89674700 & -2.47287700 & -1.05665000 \\
\hline $\mathrm{H}$ & -4.85057600 & -3.89101400 & -0.88038800 \\
\hline $\mathrm{H}$ & -5.54103300 & -3.08887400 & 0.57240800 \\
\hline O & -0.22170000 & -1.92274700 & 0.97858400 \\
\hline $\mathrm{C}$ & -0.52191700 & -1.36291900 & 2.07424500 \\
\hline O & -1.45311000 & -0.51074200 & 2.22107300 \\
\hline $\mathrm{C}$ & 0.30072700 & -1.68938500 & 3.30232900 \\
\hline $\mathrm{H}$ & -0.14739100 & -1.26830400 & 4.20348000 \\
\hline $\mathrm{H}$ & 0.39884300 & -2.77473200 & 3.39802700 \\
\hline $\mathrm{H}$ & 1.30912800 & -1.27576200 & 3.16878700 \\
\hline Ag & -1.02446900 & -1.29677200 & -1.28869900 \\
\hline $\mathrm{C}$ & 3.52798000 & -0.38681400 & -1.34215500 \\
\hline $\mathrm{C}$ & 5.93746400 & 0.72821700 & -2.27001300 \\
\hline $\mathrm{C}$ & 4.62352000 & -0.22756300 & -0.45771100 \\
\hline $\mathrm{C}$ & 3.66473900 & 0.03676000 & -2.67364300 \\
\hline $\mathrm{C}$ & 4.85317600 & 0.58989900 & -3.13928500 \\
\hline $\mathrm{C}$ & 5.81648300 & 0.31905200 & -0.94285800 \\
\hline $\mathrm{H}$ & 2.81073400 & -0.07837300 & -3.33193200 \\
\hline $\mathrm{H}$ & 4.93413000 & 0.90919000 & -4.17409700 \\
\hline $\mathrm{H}$ & 6.64383200 & 0.43191500 & -0.24878600 \\
\hline $\mathrm{H}$ & 6.87193300 & 1.15718400 & -2.62066800 \\
\hline $\mathrm{C}$ & 2.19874200 & -0.96630100 & -0.96192500 \\
\hline O & 1.24494400 & -0.93005900 & -1.75223700 \\
\hline
\end{tabular}


1.92634500

$-1.71942200$

\section{$m$-TS4-cf5}

$\mathrm{Pd}$
$\mathrm{C}$
$\mathrm{C}$
$\mathrm{C}$
$\mathrm{C}$
$\mathrm{C}$
$\mathrm{C}$
$\mathrm{H}$
$\mathrm{H}$
$\mathrm{H}$
$\mathrm{H}$
$\mathrm{H}$
$\mathrm{C}$
$\mathrm{O}$
$\mathrm{O}$
$\mathrm{C}$
$\mathrm{O}$
$\mathrm{C}$
$\mathrm{O}$
$\mathrm{C}$
$\mathrm{H}$
$\mathrm{H}$
$\mathrm{H}$
$\mathrm{H}$

2.15239300

1.56733500

0.17933000

$-0.35245500$

0.55437100

1.93647200

2.44706900

1.69669200

$-0.48658800$

0.17343200

2.60994800

3.51741200

0.56682700

1.28191800

0.62947700

$-0.49276700$

2. 56951500

1.64576000

0.58507400

1.84908000

$-0.76869100$

$-0.14943300$

$-1.36519800$

2.83025000
0.20179400

1.96141500

2. 02234000

2. 73021500

3. 33250800

3. 26114800

2. 58978500

2.26963100

1.55700900

3.88301200

3.74416300

2.54439900

1.65941500

2.63341800

0.48719000

1.89877500

$-1.61509900$

$-2.48611900$

$-2.45620100$

$-3.67172700$

0.96601400

2. 64016300

2.30085800

$-3.64320700$
0.82792800 $-0.21307500$

$-0.47115600$

$-1.54891800$

$-2.43482200$

$-2.22623200$

$-1.11754100$

1.07757700

0.24126200

$-3.29299900$

$-2.92963800$

$-0.94410100$

2.70793600

2.32994500

2.19775400

3.75366500

1.78774000

1.80746400

1.11356100

2.73370700

4.24903100

4.47814000

3.22429600

3.20995300 
$\mathrm{H}$

$\mathrm{H}$

O

C

O

C

$\mathrm{H}$

$\mathrm{H}$

$\mathrm{H}$

Ag

C

C

C

C

C

C

$\mathrm{H}$

$\mathrm{H}$

$\mathrm{H}$

$\mathrm{H}$

C

O

O

$\mathrm{H}$

C

$\mathrm{H}$

$\mathrm{H}$

C

$\mathrm{H}$

$\mathrm{H}$

C

O

$\mathrm{N}$

C

$\mathrm{H}$

$\mathrm{H}$

$\mathrm{H}$
1.06649100

1.72943900

3.79349200

3. 83273700

2. 89653100

5.16605300

5.34484000

5.11611100

5.98125500

0.91284600

$-3.57832700$

$-6.32096400$

$-4.34760700$

$-4.18341800$

$-5.55043700$

$-5.71690900$

$-3.56626200$

$-6.01114500$

$-6.29447600$

$-7.38623200$

$-2.10913600$

$-1.37088700$

$-1.70936400$

$-0.74694400$

$-1.84870500$

$-2.13362500$

$-2.06270400$

$-2.74784300$

$-2.32864800$

$-3.73304000$

$-2.96474400$

$-2.46125300$

$-3.77178100$

$-3.92561900$

$-3.07883900$

$-3.96701100$

$-4.85104500$
$-3.65557400$

$-4.59915600$

0.01272300

$-0.56737400$

$-1.16620500$

$-0.46811100$

$-1.37665400$

0.37571700

$-0.29124900$

$-1.48998100$

$-1.30020300$

$-1.17326000$

$-0.24265400$

$-2.25794300$

$-2.20775500$

$-0.19033300$

$-3.04385500$

$-2.96784900$

0.64048300

$-1.12050000$

$-1.43297000$

$-1.75325600$

$-1.20273900$

$-1.48944300$

2.93285200

3. 84862100

3.10445000

1.78826500

0.82889700

1.85350700

1.78695100

2.63692000

0.79900200

0.71608400

0.17620200

1.72385700

0.18225200
3.50043000

2.16494100

$-0.38218900$

$-1.51939800$

$-2.10506900$

$-2.24198100$

$-2.82142600$

$-2.94062700$

$-1.53802100$

$-1.21463600$

$-0.49983600$

$-1.04832600$

0.01677400

$-1.32494500$

$-1.58484000$

$-0.26534000$

$-1.74787400$

$-2.20860400$

0.12923500

$-1.25405600$

$-0.26314800$

$-1.20249900$

0.96728600

1.09540100

$-1.72097200$

$-1.18782800$

$-2.78257600$

$-1.22834700$

$-1.54902600$

$-1.70771500$

0.29047200

1. 02190100

0.80745600

2.26353700

2.70030300

2.67870400

2.49073600

\section{$m$-TS4-cf6}

C

$\mathrm{H}$

$\mathrm{H}$

C
$-1.49373700$

$-1.27852800$

$-1.85161600$

$-2.64144000$
$-3.18463100$

1.58111300

$-3.16231600$

2. 65570600

$-4.18886200$

1. 33362800

$-2.17900100$

1. 30225300 
$-2.31174200$

$-3.47303800$

$-3.15637900$

$-3.14583400$

$-3.58249300$

$-4.03494200$

$-3.49636700$

$-5.08912300$

$-4.05098100$

$-5.61702300$

$-5.50330900$

$-5.10420700$

$-3.62676200$

$-6.43637300$

$-5.51528300$

$-3.96452700$

$-4.11868800$

$-3.16099200$

$-4.87886200$

$-2.28311000$

$-1.86794500$

$-1.67651300$

2.41345900

0.39550700

$-0.31787100$

$-0.82604900$

0.93886500

$-1.23091900$

$-1.64021900$

$-0.69450900$

$-2.07043000$

4. 92562400

4.21114600

4.50024700

6.38081800

6.98433900

6.66907600

6.56794800

1. 75598000

2.57685300

0.94283400

1.74757600

1.80778900

0.79269900
$-1.15944600$

$-2.40733900$

$-2.28834700$

$-3.35942700$

$-1.13956100$

0.02164100

1. 31288000

$-0.12945900$

2. 40248300

0.96036200

$-1.12252600$

2. 23747500

3. 38636600

0.81234900

3.09848600

$-1.27859200$

$-0.28605300$

$-1.77662100$

$-1.87605100$

1.53833700

0.74047700

2.69272000

0.42107300

0.00466400

0.81757500

0.32756600

0.91427600

1. 31311500

0.44365800

1. 94122200

1.85686200

$-0.86399400$

0.06142500

$-1.74529200$

$-0.92290900$

$-0.56740200$

$-1.95748600$

$-0.28778500$

2.99976200

2.47656800

2. 38293100

4. 51837500

4.84530700

4.87521900
1.50282400

1.98170700

$-0.13439400$

$-0.73962200$

$-0.76918200$

$-0.07655800$

$-0.29236200$

0.83397700

0.39771900

1. 52296800

0.98034000

1.29321900

0.23508100

2.22089500

1.81190000

$-2.18055100$

$-2.59981900$

$-2.72441100$

$-2.27548500$

$-1.13151500$

$-1.97304300$

$-0.86220000$

0.68263200

$-1.40778600$

1.74367200

0.71787000

1.99031600

2.85369000

3.38239800

3. 56677100

2.41236500

0.20123700

$-0.29654600$

1.02053700

$-0.19696300$

0.64584900

$-0.40003000$

$-1.06372300$

$-0.52727600$

0.28965900

$-1.28085000$

$-0.58721000$

$-1.62948700$

$-0.18313200$ 
$\mathrm{H}$

C

C

C

C

$\mathrm{H}$

C

$\mathrm{H}$

C

$\mathrm{H}$

$\mathrm{H}$

$\mathrm{H}$

$\mathrm{H}$

\section{m-TS4-cf7}

C

$\mathrm{H}$

$\mathrm{H}$

C

$\mathrm{H}$

$\mathrm{H}$

C

$\mathrm{O}$

$\mathrm{N}$

C

C

C

C

C

$\mathrm{H}$

C

$\mathrm{H}$

$\mathrm{H}$

$\mathrm{H}$

C

$\mathrm{H}$

$\mathrm{H}$

$\mathrm{H}$

C

O

$O$

$\mathrm{Pd}$

$\mathrm{H}$
2.56658200

$-0.23153900$

0.81023200

$-0.13843500$

1. 90388200

0.73787900

0.93754200

$-0.93734700$

1. 94350200

3.23405600

1.00143400

2.79666600

$-0.74741500$
4.94478800

$-2.87739000$

$-2.11348800$

$-3.26892500$

$-1.66997500$

$-1.79447400$

$-2.85839000$

$-3.85992200$

$-2.05373100$

$-1.63841800$

$-3.17936500$

$-1.76852100$

2.68767400
$-0.00625400$

0.79798500

1.35014400

$-0.54553300$

0.58182200

2.38673000

$-1.34091000$

$-0.97731800$

$-0.78993800$

1.03621500

$-2.37775600$

$-1.40061700$

$-1.23369300$
1.52736900

1. 98871200

1.16169500

2.63338500

2.76632500

3. 59787900

2. 33827700

1.21866600

3. 38067800

4.51249500

4. 35235600

5.77959300

5.45659200

6.88466300

5.88369700

6.72238000

5.31463600

7.86784200

7.57914000

3.13594100

2. 65152900

2.49183500

4.09645900

2.99304000

2. 26606800

2.65094700

$-2.67652900$

1. 64954900
2.65272400

3. 64424600

2. 38619400

1. 65989900

1.69818100

1.95264200

0.22164100

$-0.12413900$

$-0.66728600$

$-0.45051600$

$-0.39157600$

$-0.28007100$

$-0.11418400$

$-0.04829200$

$-0.31692200$

0.04133000

$-0.03478400$

0.07958300

0.23689700

$-2.04348800$

$-2.62194300$

$-2.01883000$

$-2.49880200$

$-0.61360900$

$-1.52443000$

0.28441600

0.07771400

0.18945000
$-1.71037300$

$-1.79065000$

$-2.70661800$

$-1.29416200$

$-0.20679800$

$-1.72585100$

$-1.71808900$

$-2.12223100$

$-1.68351500$

$-0.82881300$

0.57013800

$-1.38869700$

1.38143200

$-0.56798800$

$-2.46913500$

0.81667900

2.45462100

$-1.01159700$

1.45476700

$-2.13534800$

$-1.34264100$

$-3.01480600$

$-2.38763000$

1.15720000

0.77175100

2.06731400

0.38735000

2.23942600 


\begin{tabular}{|c|c|c|c|}
\hline Ag & -0.08496700 & -1.14364100 & -0.16509600 \\
\hline $\mathrm{C}$ & -0.91313300 & 0.32066500 & 2.73036300 \\
\hline 0 & 0.08238400 & 0.12088100 & 1.98417200 \\
\hline 0 & -2.12921400 & 0.27488300 & 2.35920600 \\
\hline $\mathrm{C}$ & -0.67571900 & 0.69087700 & 4.18247600 \\
\hline $\mathrm{H}$ & -0.34387600 & 1.73556800 & 4.21597900 \\
\hline $\mathrm{H}$ & -1.58851400 & 0.58849700 & 4.77121300 \\
\hline $\mathrm{H}$ & 0.12074800 & 0.07029900 & 4.60284500 \\
\hline $\mathrm{C}$ & -3.96918900 & 0.85587100 & -2.07406500 \\
\hline 0 & -3.41322700 & -0.14511300 & -1.52687700 \\
\hline 0 & -3.93246800 & 2.04322000 & -1.61527500 \\
\hline $\mathrm{C}$ & -4.74108800 & 0.61503100 & -3.35036900 \\
\hline $\mathrm{H}$ & -5.81083100 & 0.60824900 & -3.11213700 \\
\hline $\mathrm{H}$ & -4.55926500 & 1.43035600 & -4.05486500 \\
\hline $\mathrm{H}$ & -4.46765000 & -0.34649300 & -3.78705100 \\
\hline $\mathrm{C}$ & -2.67004900 & -2.88271100 & 0.33294700 \\
\hline 0 & -3.31664100 & -1.86672700 & 0.76417900 \\
\hline 0 & -1.52828700 & -2.89493500 & -0.18909000 \\
\hline C & -3.41156800 & -4.20710200 & 0.45503100 \\
\hline $\mathrm{H}$ & -3.97713700 & -4.37268700 & -0.46966700 \\
\hline $\mathrm{H}$ & -2.69939400 & -5.02688600 & 0.57348300 \\
\hline $\mathrm{H}$ & -4.11714400 & -4.18356700 & 1.28837800 \\
\hline $\mathrm{C}$ & 0.36860200 & 2.73112900 & -0.73540300 \\
\hline $\mathrm{C}$ & 0.44179900 & 3.57512800 & 0.38197800 \\
\hline $\mathrm{C}$ & -0.79429100 & 1.97461800 & -0.90916400 \\
\hline $\mathrm{C}$ & -0.61772800 & 3.67511600 & 1.28922500 \\
\hline $\mathrm{H}$ & 1.33622800 & 4.17643200 & 0.53589600 \\
\hline $\mathrm{C}$ & -1.86480100 & 2.02287900 & 0.01169400 \\
\hline $\mathrm{H}$ & -0.87009200 & 1.33154600 & -1.78197300 \\
\hline $\mathrm{C}$ & -1.76288800 & 2.90352400 & 1.10820300 \\
\hline $\mathrm{H}$ & -3.04852900 & 2.02919900 & -0.68471700 \\
\hline $\mathrm{H}$ & -2.57503500 & 2.95724800 & 1.82726300 \\
\hline $\mathrm{H}$ & -0.54183400 & 4.35587700 & 2.13371700 \\
\hline
\end{tabular}

\section{m-TS4-cf8}

$\mathrm{C}$
$\mathrm{H}$
$\mathrm{H}$
$\mathrm{C}$
$\mathrm{H}$
$\mathrm{H}$
$\mathrm{C}$
$\mathrm{O}$

1.88333700

2.98035200

1.52005900

1.69788200

2. 87131500

2.59492300

2.26514200

3.99239100

1. 35285200

2. 98291700

1.97187500

1.12365300

2. 59786100

0.95152200

1.20286500

3.82491200

2. 04918400

1.82535000

3. 51345900

2.21141300

$-0.29094800$

3.36341100

3.28188400

$-0.87268700$ 
4.15691200

4.70781100

4.04198200

6.01019200

4.71907000

6.66595400

6.50087500

6.01685400

4.20536000

7.67549900

6.51455300

4.53730400

4.23964300

4.01959900

5.61974800

2.61695800

1.89511200

2.20570200

$-2.56833700$

1.19087500

$-0.48534000$

$-0.35417700$

$-0.23477100$

$-1.11308500$

0.45991200

1.49805100

0.41089700

0.06234600

$-4.78227300$

$-4.29853700$

$-4.14411900$

$-6.21529400$

$-6.84942600$

$-6.32778000$

$-6.53117000$

$-3.02443100$

$-3.25879600$

$-2.34355700$

$-3.62058000$

$-3.87247200$

$-2.86197000$

$-4.49846900$

0.58367300

$-0.46362800$
1.15440900

0.05361600

$-0.89574000$

$-0.17035100$

$-1.17744000$

0.01424000

0.21092400

0.31845800

$-2.21465500$

0.68205700

$-0.82555200$

0.97948000

1.16749500

0.16350300

$-2.04830600$

1. 15518700

$-3.15631300$

0.82961000

1. 35265500

1.66567800

$-2.30362600$

$-2.81809900$

$-2.73866400$

$-2.41935600$

$-0.38522800$

$-0.85909900$

$-0.12863700$

$-2.63359300$

0.64747500

$-2.65500400$

0.00217100

$-0.65075900$

$-1.34130500$

$-1.84579000$

1.63827200

$-2.37058400$

0.49683400

$-0.86181400$

1. 91180700

$-2.40172200$

2.79244000

2.47443800

3. 66502900

3.05556000

0.42186600

1.62776200

$-0.19807600$

2. 32269200

1.27606400

2.00239900

0.12597000

1.58986700

0.91920600

3.08896300

0.13365600

1.58180300

$-0.82994200$

$-2.46740500$

$-0.97645700$

0.21299400

$-1.86215400$

$-1.88670400$

$-3.82459000$

$-1.31462600$

$-3.86773500$

$-2.37689200$

$-4.59026400$

$-1.11215500$

$-4.03432900$

$-0.70006200$

2.78590000

0.75980700

2.02266300

1.29242800 
C

C

$\mathrm{H}$

C

$\mathrm{H}$

C

$\mathrm{H}$

$\mathrm{H}$

$\mathrm{H}$
0.42473800

$-1.65110700$

$-0.35592900$

$-0.72962900$

1.22935800

$-1.75491900$

$-2.89221900$

$-0.82958300$

$-2.65712000$
3.34200900

1.78830800

1.57886800

3.10939200

3.93745000

2.33219800

1.95447700

3.54409500

2.15951000
$-0.52072800$

0.56791200

2.27878500

$-1.26858000$

$-0.93376400$

$-0.73699900$

1.15456300

$-2.26002300$

$-1.31631600$

\section{m-TS4-cf9}

$\mathrm{Pd}$

C

C

C

C

C

C

$\mathrm{H}$

$\mathrm{H}$

$\mathrm{H}$

$\mathrm{H}$

$\mathrm{H}$

C

O

O

C

O

C

O

C

$\mathrm{H}$

$\mathrm{H}$

$\mathrm{H}$

$\mathrm{H}$

$\mathrm{H}$

$\mathrm{H}$

O

C

O

C

$\mathrm{H}$

$\mathrm{H}$
$-2.56011500$

$-2.45666700$

$-1.20103700$

$-0.50595800$

$-1.09965500$

$-2.35234700$

$-3.02917100$

$-3.25105000$

$-0.79312400$

$-0.57240900$

$-2.78763200$

$-3.98339900$

$-3.02491600$

$-3.66343000$

$-2.32585900$

$-3.11875300$

$-2.39108100$

$-1.22376100$

$-0.15886700$

$-1.11235900$

$-4.03991900$

$-3.17604000$

$-2.26944600$

$-2.09539200$

$-0.51592200$

$-0.58187000$

$-3.02354500$

$-2.21420700$

$-1.00332500$

$-2.87666000$

$-2.13635800$

$-3.28920200$
0.53564800

$-1.56921800$

$-2.09372600$

$-3.03425700$

$-3.46144900$

$-2.99458400$

$-2.05341700$

$-1.45027700$

$-1.76173500$

$-4.18043600$

$-3.35538400$

$-1.65723700$

$-0.58281400$

$-1.49890000$

0.31095900

$-0.54570800$

2. 60372700

3.10196600

2.47707300

4. 57145200

$-0.01872900$

$-1.56026100$

$-0.00434500$

5.01713300

4.66235700

5.10561900

0.85419100

0.73578500

0.44274400

0.94073400

1.26896200

$-0.02038600$
0.24432300

$-0.09521100$

0.30757400

$-0.45938700$

$-1.65783000$

$-2.05899700$

$-1.28536600$

1.03062800

1.26026200

$-2.28130300$

$-2.98739800$

$-1.61822300$

2.85911600

2. 24371200

2. 29185800

4. 36662900

0.52532700

0.53308500

0.26184700

0.90346600

4. 64123600

4.76723900

4.78687800

1.06180700

1.81813800

0.10848700

$-1.71117300$

$-2.70370700$

$-2.66519300$

$-4.05894700$

$-4.79134400$

$-4.38906300$ 
$\mathrm{H}$

Ag

C

C

C

C

C

C

$\mathrm{H}$

$\mathrm{H}$

$\mathrm{H}$

$\mathrm{H}$

C

O

O

$\mathrm{H}$

C

$\mathrm{H}$

$\mathrm{H}$

C

$\mathrm{H}$

$\mathrm{H}$

C

O

$\mathrm{N}$

C

$\mathrm{H}$

$\mathrm{H}$

$\mathrm{H}$
$-3.69748300$

0.22426400

4.09836300

6.58559100

4.49207500

4.96645700

6.20491300

5.72570300

4.65203600

6.86436100

5.99739200

7.54299100

2.75939000

2.04508700

2.40504700

1.41176600

0.80028300

0.55267300

1. 37651500

1. 72113400

2. 39773100

1.16223100

2.53721200

2.18002100

3.70130800

3.97157600

3. 46857900

3.63313600

5.04993800
1.65788000

0.21764300

0.97502500

0.20132000

$-0.37718200$

1. 92042300

1.54368300

$-0.75208300$

2. 95702800

2.29396200

$-1.80339000$

$-0.10611000$

1.40535400

0.70321300

2.60963400

2.73375700

$-3.67288800$

$-4.62257200$

$-3.93870800$

$-2.89848400$

$-3.62162000$

$-2.43830000$

$-1.80753900$

$-1.32463800$

$-1.42222300$

$-1.62937500$

$-0.85265800$

$-2.61153100$

$-1.56862000$
$-3.98875400$

$-0.72611500$

0.23650500

$-0.82134300$

0.25638400

$-0.32923100$

$-0.84350100$

$-0.27654500$

$-0.36176000$

$-1.26980400$

$-0.26182500$

$-1.23237900$

0.74636100

1. 45755100

0.31024000

0.46517100

$-0.02158700$

0.47399500

$-0.91482000$

0.93603700

1.40194700

1.76093700

0.25162600

$-0.83241100$

0.85717700

2.28473600

2.87232900

2.61535200

2.44732700

\section{$m$-TS4-cf10}

$\mathrm{Pd}$
$\mathrm{C}$
$\mathrm{C}$
$\mathrm{C}$
$\mathrm{C}$
$\mathrm{C}$
$\mathrm{C}$
$\mathrm{H}$
$\mathrm{H}$
$\mathrm{C}$
$\mathrm{O}$
$\mathrm{O}$

2.38746600

0.43796700

0.74572300

1.23493500

$-2.63105400$

$-1.76987300$

0.20827100

$-3.25753800$

$-1.05431000$

0.07716400

$-3.08456000$

0.32900100

1.03037100

$-2.30001000$

0.99754200

2. 07501600

$-1.64863400$

0.31896800

2.15853000

$-1.82505900$

$-1.09194600$

3.37856400

$-1.56669300$

0.81531500

2.98505200

$-1.37748000$

$-1.63892000$

5.02166600

$-0.54522400$

0.17313100

4.65481300

$-1.57513600$

0.83129500

4.24143400

0.38576900

$-0.19845500$ 
6.48490000

2. 39049800

1. 54954800

0.81979500

1.39847500

6.63139300

7.03279700

6.87414100

2.15708900

1.45477900

0.40356700

0.79902600

$-0.43453500$

$-0.83184100$

$-1.45407600$

$-2.23580100$

$-0.99316700$

$-1.91645300$

0.47275300

$-3.70005400$

$-6.20343700$

$-4.16350200$

$-4.50857700$

$-5.75431900$

$-5.40986200$

$-4.13697100$

$-6.36429200$

$-5.73993200$

$-7.16821000$

$-2.36798900$

$-1.86256300$

$-1.80913100$

$-0.86354000$

$-1.09080200$

$-0.92662600$

$-1.14449400$

$-2.46386500$

$-3.18478100$

$-2.43074300$

$-3.02674600$

$-3.17711900$

$-3.41671100$

$-3.28359800$

$-2.24259100$
$-0.42220500$

2. 53642500

3. 11209500

2.55449700

4. 61286300

0.33469200

$-0.12393000$

$-1.39128300$

5.01085900

5.10863000

4.81164500

0.61360400

0.43698500

$-0.03253300$

0.87733800

0.11741500

1.07014200

1.79858600

0.19315500

1.18686100

0.84040000

$-0.11240400$

2.29033300

2.12600500

$-0.26083000$

3.28492100

2. 99421600

$-1.26225300$

0.69379700

1.46319800

0.77905600

2.56418800

2.66127500

$-3.70244600$

$-4.78471800$

$-3.29736900$

$-3.48016400$

$-4.15744500$

$-3.75098500$

$-2.07977100$

$-1.72174400$

$-1.30490100$

$-1.73294900$

$-1.96035200$
$-0.17909600$

0.68893800

$-0.06759200$

$-0.94475900$

0.11315600

$-0.95084400$

0.72200200

$-0.50124300$

0.78852300

$-0.86044700$

0.52915100

1.99256400

1.65675000

0.57393200

2.68777700

2.75384000

3.65796400

2.31355400

$-1.45989900$

$-0.42940200$

0.79588200

$-0.13042100$

$-0.10704900$

0.48815100

0.49007100

$-0.32787600$

0.71994600

0.74429900

1.27337800

$-1.04255900$

$-1.93452200$

$-0.55069100$

$-0.86871100$

1.07329900

1.16646400

2.08831000

0.40121400

0.87503300

$-0.65448700$

0.66508400

1.82906500

$-0.40641600$

$-1.80339100$

$-2.04407600$ 
$\mathrm{H}$

$\mathrm{H}$

$\mathrm{H}$

$\mathrm{H}$

$\mathrm{H}$
$-3.91674900$

$-3.58863700$

$-0.50304100$

1. 32832400

0.92014900
$-2.60406100$

$-0.90920400$

$-3.87741100$

$-2.78803700$

$-2.13777200$
$-2.01648500$

$-2.44430700$

$-1.59296500$

$-2.84165200$

2.06587200

\section{m-TS4-cf11}

\begin{tabular}{|c|c|}
\hline $\mathrm{Pd}$ & 2.54637500 \\
\hline $\mathrm{C}$ & 1.49779200 \\
\hline $\mathrm{C}$ & 0.14859700 \\
\hline C & -0.40991300 \\
\hline C & 0.42462000 \\
\hline $\mathrm{C}$ & 1.76994000 \\
\hline $\mathrm{C}$ & 2.30076600 \\
\hline $\mathrm{H}$ & 0.00956700 \\
\hline $\mathrm{H}$ & 2.38958100 \\
\hline $\mathrm{H}$ & 3.33643500 \\
\hline $\mathrm{C}$ & 2.95211300 \\
\hline O & 1.75824700 \\
\hline O & 3.62391300 \\
\hline C & 3.59416400 \\
\hline O & 3.53960900 \\
\hline $\mathrm{C}$ & 3.42133100 \\
\hline O & 2.57530800 \\
\hline C & 4.43809100 \\
\hline $\mathrm{H}$ & 3.52115300 \\
\hline $\mathrm{H}$ & 4.65406600 \\
\hline $\mathrm{H}$ & 3.08138100 \\
\hline $\mathrm{H}$ & 4.91577000 \\
\hline $\mathrm{H}$ & 5.20733700 \\
\hline $\mathrm{H}$ & 3.95422500 \\
\hline O & 1.55071600 \\
\hline C & 0.57798000 \\
\hline O & 0.27550500 \\
\hline C & -0.30704200 \\
\hline $\mathrm{H}$ & -1.23995900 \\
\hline $\mathrm{H}$ & 0.16358500 \\
\hline $\mathrm{H}$ & -0.55576100 \\
\hline $\mathrm{Ag}$ & 0.60744400 \\
\hline $\mathrm{C}$ & -3.90695800 \\
\hline $\mathrm{C}$ & -6.62752500 \\
\hline C & -4.54706100 \\
\hline C & -4.64694400 \\
\hline
\end{tabular}

0.35555800

2.09841600

2. 24762500

3.50441200

4. 62802700

4. 51098800

3.25506400

5.61420800

5.40110400

3.15943700

0.27681500

0.72797200

0.21746400

$-0.26291300$

$-1.39873000$

$-2.43047100$

$-2.62028600$

$-3.53484400$

$-1.35608700$

0.00116300

0.10122300

$-3.42022000$

$-3.47455700$

$-4.51168700$

0.55461900

$-0.21421300$

$-1.26433700$

0.22212200

0.60000500

1.01098200

$-0.63477700$

$-1.57905700$

$-1.40993200$

$-1.58295500$

$-0.32458900$

$-2.55243300$
$-0.47951800$

0.15710500

$-0.22802800$

$-0.47773400$

$-0.37965900$

$-0.01814200$

0.26313600

$-0.57871300$

0.05517600

0.58036700

2.35354600

2. 41990300

1. 28148200

3. 60633400

$-1.05925400$

$-0.32166900$

0.59950400

$-0.56517900$

3.56354700

3. 62826700

4.49759300

$-1.53989400$

0.21376900

$-0.48434300$

$-2.26112700$

$-2.55024300$

$-1.91589100$

$-3.69924500$

$-3.26063400$

$-4.28774600$

$-4.33130000$

0.57061400

$-0.00594800$

0.63025600

0.61704200

$-0.33623300$ 


$\begin{array}{lrrr}\mathrm{C} & -5.99652200 & -2.65202700 & -0.00698500 \\ \mathrm{C} & -5.91105700 & -0.42291200 & 0.91949000 \\ \mathrm{H} & -4.14248700 & -3.36761200 & -0.84437900 \\ \mathrm{H} & -6.55137500 & -3.55236800 & -0.25315400 \\ \mathrm{H} & -6.41607500 & 0.42750100 & 1.36806300 \\ \mathrm{H} & -7.68283700 & -1.63859200 & 0.88197300 \\ \mathrm{C} & -2.44335500 & -1.40736800 & -0.29246500 \\ \mathrm{O} & -1.62543100 & -1.02199100 & 0.55930000 \\ \mathrm{O} & -2.14186100 & -1.90958600 & -1.46326500 \\ \mathrm{H} & -1.16122600 & -1.72587900 & -1.70835300 \\ \mathrm{C} & -1.87962300 & 3.65955400 & -0.81473400 \\ \mathrm{H} & -2.11559300 & 4.72563200 & -0.90114600 \\ \mathrm{H} & -2.09804300 & 3.20092700 & -1.78526100 \\ \mathrm{C} & -2.81043000 & 3.02858600 & 0.23894300 \\ \mathrm{H} & -3.75710200 & 3.58563300 & 0.29059200 \\ \mathrm{H} & -2.35717600 & 3.11233600 & 1.23155700 \\ \mathrm{C} & -3.18524000 & 1.57735800 & -0.07282300 \\ \mathrm{O} & -2.94864300 & 1.06997300 & -1.16584000 \\ \mathrm{~N} & -3.87407800 & 0.90102500 & 0.91685200 \\ \mathrm{C} & -4.03124400 & 1.45607000 & 2.26569700 \\ \mathrm{H} & -3.05423400 & 1.68786000 & 2.69981100 \\ \mathrm{H} & -4.65144500 & 2.36032200 & 2.27863300 \\ \mathrm{H} & -4.50160600 & 0.70143700 & 2.89587400 \\ \mathrm{H} & 1.56895300 & 1.31962000 & 1.27123700 \\ \mathrm{H} & -0.47547600 & 1.36213300 & -0.31378900\end{array}$

\section{m-TS4-cf12}

$\mathrm{Pd}$
$\mathrm{C}$
$\mathrm{C}$
$\mathrm{C}$
$\mathrm{C}$
$\mathrm{C}$
$\mathrm{C}$
$\mathrm{H}$
$\mathrm{H}$
$\mathrm{C}$
$\mathrm{O}$
$\mathrm{O}$
$\mathrm{C}$
$\mathrm{O}$
$\mathrm{C}$
$\mathrm{O}$

$$
\begin{array}{r}
2.69711200 \\
0.28865500 \\
-0.68047000 \\
-0.47818200 \\
0.74153500 \\
1.73478100 \\
1.48276700 \\
3.08024700 \\
2.23692300 \\
4.76712000 \\
4.29865100 \\
4.20359500 \\
6.08475900 \\
3.33011800 \\
2.99234900 \\
2.22998000
\end{array}
$$$$
-0.01960200
$$$$
0.56861800
$$$$
-2.46743900
$$$$
-1.81735200
$$$$
-2.91489400
$$$$
-0.91633200
$$$$
-2.84176200
$$$$
0.46791200
$$$$
-2.33343100
$$$$
0.93176700
$$$$
-1.86570100
$$$$
0.05477800
$$$$
-1.93541100
$$$$
-1.33798500
$$$$
-2.15092500
$$$$
0.24719900
$$$$
-1.58063200
$$$$
-2.03538700
$$$$
-1.50509300
$$$$
-0.71152600
$$

$-2.48652300$

$-0.04732700$

$-0.36965200$

$-0.81010300$

$-1.69775500$

$-1.42409700$

1.95358500

0.68827400

2.71306000

$-0.28549200$

2.42552500

$-1.24479400$ 
3.56844100

6.24497400

6.88829600

6.11013300

4.49219600

3.73834200

2.83283000

1.44444100

0.44890900

0.16751900

$-0.47769000$

$-1.36327900$

0.00250500

$-0.80608200$

0.44202300

$-4.12965300$

$-6.90418100$

$-4.80618500$

$-4.85950500$

$-6.23482600$

$-6.19534300$

$-4.32590200$

$-6.77894800$

$-6.72566600$

$-7.97953000$

$-2.64656700$

$-1.86872200$

$-2.29917600$

$-1.30204600$

$-1.53929800$

$-1.38411300$

$-1.42266400$

$-2.98678600$

$-3.66377500$

$-3.17662500$

$-3.36184900$

$-3.02455100$

$-4.15041400$

$-4.48280500$

$-3.57580600$

$-5.16584800$

$-4.95831600$

$-1.60491000$

0.10378300
4.11934300

$-0.90499200$

$-1.66495600$

$-2.68124600$

4.14598600

4.48985100

4.77361300

0.16192600

0.95665500

1.79910600

0.80262700

0.25329900

0.24907800

1.78505700

1.03591500

1.15550700

1.13603000

$-0.06912400$

2.35167300

2.35131100

$-0.06070200$

3.28642400

3.29000800

$-1.00606400$

1.11424000

1.25065900

0.60753300

2.13189600

2.08213200

$-3.27309800$

$-4.32490100$

$-2.68462100$

$-3.11544100$

$-3.46699100$

$-3.75412100$

$-1.64942100$

$-0.80053900$

$-1.33972900$

$-2.31916900$

$-2.78441800$

$-3.10077000$

$-1.79071000$

$-3.32403100$

$-2.52796800$
$-0.23291700$

$-2.15607100$

$-0.67956000$

$-1.90005500$

0.34861100

$-1.24669100$

0.25010300

2.18541100

2. 22749700

1.33143900

3.41549100

3.07060400

4.22409500

3.76496900

$-1.03306100$

$-0.29222000$

$-0.69171700$

$-0.42688400$

$-0.32890500$

$-0.54541300$

$-0.61353300$

$-0.19356800$

$-0.58883600$

$-0.67912900$

$-0.84440300$

$-0.13263500$

$-0.85148300$

0.77499300

1.00012400

1.46467400

1.74229000

2.37970400

0.97354800

1.76381900

0.11036100

0.74377700

1.56308100

$-0.35012700$

$-1.39122600$

$-1.78306700$

$-1.03740300$

$-2.21761900$

$-1.30997200$

$-2.88678500$ 


\section{m-TS4-cf13}

\begin{tabular}{|c|c|c|c|}
\hline $\mathrm{Pd}$ & 1.93033000 & -0.64407600 & 0.98276600 \\
\hline C & 1.62654600 & 1.42982600 & 1.36582700 \\
\hline $\mathrm{C}$ & 0.46251700 & 1.97637000 & 0.78023300 \\
\hline $\mathrm{C}$ & 0.40203400 & 3.29783500 & 0.33013000 \\
\hline $\mathrm{C}$ & 1.56752700 & 4.07102000 & 0.43535300 \\
\hline $\mathrm{C}$ & 2.73856200 & 3.55712800 & 1.00493000 \\
\hline $\mathrm{C}$ & 2.77120100 & 2.24801600 & 1.47571800 \\
\hline $\mathrm{H}$ & 1.18688900 & 0.80446700 & 2.46869500 \\
\hline $\mathrm{H}$ & -0.43156500 & 1.36851400 & 0.74402100 \\
\hline $\mathrm{H}$ & 1.55505700 & 5.10149200 & 0.08526400 \\
\hline $\mathrm{H}$ & 3.61953400 & 4.18920200 & 1.08331600 \\
\hline $\mathrm{H}$ & 3.67880900 & 1.84739300 & 1.91594500 \\
\hline $\mathrm{C}$ & -0.37785200 & -0.50564900 & 2.72866900 \\
\hline O & 0.28315600 & 0.37405900 & 3.35862600 \\
\hline 0 & 0.06113500 & -1.12936400 & 1.70597800 \\
\hline $\mathrm{C}$ & -1.81042300 & -0.76812900 & 3.12252000 \\
\hline O & 2.28058100 & -2.66632400 & 0.54119400 \\
\hline $\mathrm{C}$ & 1.61832500 & -3.26949500 & -0.35216400 \\
\hline 0 & 0.77775600 & -2.73657900 & -1.14422400 \\
\hline $\mathrm{C}$ & 1.86053500 & -4.76233400 & -0.49060000 \\
\hline $\mathrm{H}$ & -2.12151800 & -1.76570600 & 2.80786400 \\
\hline $\mathrm{H}$ & -1.94560700 & -0.63159400 & 4.19716800 \\
\hline $\mathrm{H}$ & -2.42107700 & -0.02420000 & 2.59341400 \\
\hline $\mathrm{H}$ & 2.62588600 & -5.10955000 & 0.20499200 \\
\hline $\mathrm{H}$ & 0.92055700 & -5.29326200 & -0.30380000 \\
\hline $\mathrm{H}$ & 2.15890900 & -4.98165100 & -1.52083600 \\
\hline 0 & 3.73773800 & -0.16982400 & 0.14552900 \\
\hline $\mathrm{C}$ & 3.83882000 & 0.30385100 & -1.04028900 \\
\hline $\mathrm{O}$ & 2.92505800 & 0.44737600 & -1.88985500 \\
\hline $\mathrm{C}$ & 5.23437500 & 0.77954400 & -1.40852600 \\
\hline $\mathrm{H}$ & 5.38355900 & 0.71794400 & -2.48838600 \\
\hline $\mathrm{H}$ & 5.32410400 & 1.82967800 & -1.10496800 \\
\hline $\mathrm{H}$ & 5.99501300 & 0.20328700 & -0.87761800 \\
\hline Ag & 0.82750800 & -0.26954600 & -1.61841600 \\
\hline $\mathrm{C}$ & -3.54712100 & -0.98988100 & -0.28252300 \\
\hline $\mathrm{C}$ & -6.18696200 & -0.63636200 & 0.62062400 \\
\hline C & -4.20076200 & 0.25082000 & -0.45223900 \\
\hline $\mathrm{C}$ & -4.24324500 & -2.03685500 & 0.34455200 \\
\hline $\mathrm{C}$ & -5.55030700 & -1.86628500 & 0.79202500 \\
\hline $\mathrm{C}$ & -5.50916000 & 0.41333100 & 0.00105100 \\
\hline
\end{tabular}




\section{m-TS4-cf14}

$\begin{array}{ll}\mathrm{C} & -1.05474100 \\ \mathrm{H} & -0.52826400 \\ \mathrm{H} & -1.66395900 \\ \mathrm{C} & -1.98697600 \\ \mathrm{H} & -1.39136000 \\ \mathrm{H} & -2.66319500 \\ \mathrm{C} & -2.85510500 \\ \mathrm{O} & -3.10684600 \\ \mathrm{~N} & -3.32465100 \\ \mathrm{C} & -4.19778600 \\ \mathrm{C} & -3.85830500 \\ \mathrm{C} & -5.39671000 \\ \mathrm{C} & -4.71800000 \\ \mathrm{C} & -6.25567700 \\ \mathrm{H} & -5.63660800 \\ \mathrm{C} & -5.91793200 \\ \mathrm{H} & -4.43111000 \\ \mathrm{H} & -7.18510100 \\ \mathrm{H} & -6.58103700 \\ \mathrm{C} & -3.39095100\end{array}$

$$
\begin{aligned}
& -3.74187600 \\
& -6.06745900 \\
& -5.98097000 \\
& -7.20489400 \\
& -2.13948100 \\
& -1.46581600 \\
& -1.70311200 \\
& -0.72709400 \\
& -0.88696600 \\
& -1.49057300 \\
& -0.64038200 \\
& -1.76771500 \\
& -1.15097300 \\
& -2.38963900 \\
& -2.67530000 \\
& -2.61848400 \\
& -3.57290300 \\
& -3.59115900 \\
& -3.57799000 \\
& -2.74205200 \\
& -4.52037800
\end{aligned}
$$

$-2.98794100$

0.47696800

$-2.69012900$

1.27487600

1.38180500

$-0.13101100$

$-0.49093600$

0.97108100

$-1.22685200$

$-0.36003700$

$-2.43482600$

$-2.54712100$

3. 92386200

4.21314300

4.84856000

3.05950500

2.47697200

3.71800100

2.08759200

1.94150200

1. 36905700

1. 37610300

2.39986600

0.82100600

0.90787400

$-0.72528500$

$-1.30468100$

$-0.45862500$

$-0.71358500$

$-0.17051800$

0.69917800

$-0.70492600$

$-1.08995100$

$-1.78820300$

$-1.70905800$

$-0.34021600$

0.87878900

$-1.09876600$

$-2.56234600$

$-2.94255700$

$-2.97707200$

$-2.89477800$
$-3.73248300$

0.26838700

$-4.35556800$

1.00142700

$-4.39626800$

$-0.35212600$

$-2.77768700$

1. 05116800

$-2.15857800$

1.72510100

$-3.36971400$

1.68552100

0.12928400

$-1.02034200$

0.65172300

$-0.17564500$

$-0.57385400$

$-0.63253000$

$-1.42208700$

$-1.45419300$

$-0.35268400$

$-1.85146700$

1.54531500

$-1.73298300$

$-1.79103100$

$-0.20072500$

2.11309200

$-2.49744300$

$-0.48499800$

2.10981300 
H

$\mathrm{H}$

$\mathrm{H}$

C

O

0

Pd

$\mathrm{H}$

Ag

C

O

O

C

$\mathrm{H}$

H

$\mathrm{H}$

C

o

O

C

$\mathrm{H}$

H

$\mathrm{H}$

C

O

O

C

H

$\mathrm{H}$

$\mathrm{H}$

C

C

C

C

$\mathrm{H}$

C

$\mathrm{H}$

C

$\mathrm{H}$

$\mathrm{H}$

$\mathrm{H}$

$\begin{array}{rrr}-3.81140500 & 0.50383500 & 2.27749200 \\ -4.03067600 & -1.23685200 & 2.59031500 \\ -2.39589800 & -0.49840500 & 2.55475100 \\ -2.59874900 & 2.03169100 & -0.11030100 \\ -2.13690300 & 1.85694000 & 1.01508700 \\ -1.98947000 & 2.78741100 & -1.02910200 \\ 2.58867400 & 0.16229600 & 0.21032900 \\ -1.07842900 & 3.01024800 & -0.68583500 \\ -0.24049800 & 0.37922100 & 0.33711500 \\ 1.15288600 & -0.70120800 & 2.81778300 \\ -0.03006100 & -0.53538100 & 2.44063800 \\ 2.20839700 & -0.53625700 & 2.11471500 \\ 1.41739800 & -1.13689400 & 4.25048000 \\ 0.50538000 & -1.53275500 & 4.70116300 \\ 2.21837300 & -1.87992300 & 4.28103400 \\ 1.75046000 & -0.26491100 & 4.82438600 \\ 4.57996200 & -0.14212800 & -1.76848600 \\ 3.71362200 & 0.73296000 & -1.44260600 \\ 4.54710600 & -1.36398600 & -1.40640700 \\ 5.72852800 & 0.30300300 & -2.64190100 \\ 6.62268400 & 0.39536800 & -2.01497700 \\ 5.93047700 & -0.45400000 & -3.40373800 \\ 5.51231000 & 1.26963400 & -3.09868000 \\ 1.61976300 & 2.91932700 & 0.40656200 \\ 2.55995300 & 2.18546500 & 0.85411700 \\ 0.61201200 & 2.52009900 & -0.25206000 \\ 1.71977200 & 4.40447100 & 0.70738900 \\ 1.66826000 & 4.96647600 & -0.23082300 \\ 0.85856000 & 4.69890600 & 1.31740900 \\ 2.64467800 & 4.64267100 & 1.23430900 \\ -0.05567400 & -2.99256200 & -0.60225400 \\ 1.13651400 & -2.46901400 & -0.07608800 \\ -0.36704400 & -2.73208300 & -1.94617500 \\ 1.96935000 & -1.61177200 & -0.83382100 \\ 1.39788100 & -2.68041900 & 0.95570500 \\ 0.45627000 & -1.92412900 & -2.72936600 \\ -1.28690600 & -3.13066200 & -2.35720300 \\ 1.58817900 & -1.33956200 & -2.17123300 \\ 3.38083800 & -1.56266500 & -0.87590100 \\ 0.19444800 & -1.72550700 & -3.76522900 \\ 2.18427100 & -0.66243600 & -2.77263700\end{array}$

$-3.81140500$

0.50383500

2.27749200

2.59031500

$-0.11030100$

.

$-1.02910200$

$-0.68583500$

0.33711500

(1778300

2.11471500

.

4.28103400

.82438600

$-1.44260600$

$-1.40640700$

$-2.64190100$

$-2.01497700$

$-3.09868000$

0.40656200

0.85411700

0.70738900

$-0.23082300$

1.23430900

$-0.60225400$

$-0.07608800$

$-1.94617500$

0.95570500

$-2.72936600$

$-2.35720300$

$-2.17123300$

$-0.87590100$

$-2.77263700$

\section{$m$-TS4-cf15}


$-2.28038900$

$-2.28929100$

$-2.87020800$

$-3.02108200$

$-2.38960700$

$-3.87560400$

$-3.48803200$

$-3.02567600$

$-4.44642300$

$-4.68864600$

$-3.68636900$

$-5.95349500$

$-3.96819400$

$-6.23637500$

$-6.70710600$

$-5.23920700$

$-3.17527600$

$-7.22858400$

$-5.44741900$

$-4.89183800$

$-5.71320900$

$-5.26510200$

$-4.08525900$

$-2.31512200$

$-1.34588400$

$-2.25098400$

2. 48164400

$-1.29559600$

0.92670900

0.70579800

0.18049500

1.68852500

0.12004300

$-0.93277700$

0.66178700

0.14781300

2. 61744800

3.39093700

1. 36229800

3.22790200

4.20919100

2.56928300

3.37306600

3.92004500
3.65038700

4.60652000

3.80898500

2. 62088100

2.25868800

3.11569700

1. 40757200

1.15408700

0.59813000

$-0.67531100$

$-1.66223500$

$-0.92935600$

$-2.86647700$

$-2.14991700$

$-0.14990000$

$-3.11912300$

$-3.60149400$

$-2.33292600$

$-4.06402100$

0.75761800

0.06090300

1.76926900

0.53132400

$-1.49106700$

$-2.06780400$

$-0.73370500$

0.68402900

$-0.60724100$

$-1.80264300$

0.76969800

$-0.29900000$

1. 36599800

1.40665800

1.64934500

2. 31174800

0.68408800

$-0.38486100$

0.04180200

$-0.16085800$

$-1.24276100$

$-0.85168500$

$-1.30375000$

$-2.24524000$

$-1.77614600$
0.16410500

$-0.37553200$

1.07593900

$-0.69902100$

$-1.52345900$

$-1.17316000$

0.09789400

1.20357300

$-0.48654300$

0.12962000

0.22613000

0.65940900

0.88203300

1.27501500

0.59885600

1.39343200

0.97280700

1.67786900

1.88640100

$-1.87258600$

$-2.04985500$

$-2.05231600$

$-2.57970500$

$-0.33226700$

0.17118500

$-1.41239000$

$-0.18050600$

$-1.73740200$

$-0.17684200$

$-2.47947000$

$-2.04848900$

$-1.94639000$

$-3.72554600$

$-3.53902500$

$-4.00278700$

$-4.54727100$

2.47104800

1.55957100

2. 49977800

3.55059900

3. 82974800

4.41785600

3.13109600

$-0.90297000$ 
3. 85147700

3.05142500

5.21062200

5.92320600

5.03064200

5.64868400

$-0.84602700$

$-0.13704500$

$-0.19228600$

1.16259300

$-0.62733000$

1.12470100

$-0.70764100$

1.78819100

1.17582400

2.78880400

1.67537100
$-0.53854600$

$-2.46231200$

$-2.47386400$

$-2.37724900$

$-3.53628100$

$-2.00070300$

3.36489100

4.40682100

2.14502400

4.23448600

5.37031500

1.93583100

1.31600900

2.99895900

0.79474400

2.84266700

5.05859000
$-1.20154200$

$-0.29519200$

$-1.30293400$

$-0.47495500$

$-1.48131500$

$-2.18446500$

0.58028900

1.20440000

0.40769900

1. 67970300

1.33442200

0.88234400

$-0.06317600$

1.53086500

1.62499700

1.92648300

2.16899600

\section{m-TS4-cf16}

C

$\mathrm{H}$

$\mathrm{H}$

C

$\mathrm{H}$

$\mathrm{H}$

C

O

$\mathrm{N}$

C

C

C

C

C

$\mathrm{H}$

C

$\mathrm{H}$

$\mathrm{H}$

$\mathrm{H}$

C

$\mathrm{H}$

$\mathrm{H}$

$\mathrm{H}$

C
1.86779200

1.37038400

2. 45846000

2.84016700

2.28170300

3. 42240300

3. 79116600

3.88484200

4.51764500

4.94907700

4.01487800

6.29311900

4.44691900

6.72084900

6.99326500

5.79520100

3.71228100

7.77224500

6.12058100

4.63297100

5.53618500

4.74054100

3. 77356800

2.57290300
3.41769200

4.02268400

4.09936700

2.45638000

1. 69296200

3.01898800

1.75369900

2.08656600

0.68164000

$-0.27716000$

$-1.11737000$

$-0.33796200$

$-1.96621100$

$-1.22114400$

0.33461800

$-2.02834300$

$-2.58701500$

$-1.25933500$

$-2.70322200$

0.34502300

$-0.25527200$

1.25446600

$-0.23200600$

$-1.18370100$
0.69944400

1.46659500

0.07935800

1. 41828900

1.97452300

2. 16100400

0.45017500

$-0.72587400$

0.93416100

$-0.04316300$

$-0.68833500$

$-0.40541500$

$-1.71301600$

$-1.39906500$

0.08071100

$-2.06192100$

$-2.21588800$

$-1.66984400$

$-2.84807000$

2.35082200

2.48848600

2.94751400

2.70810100

$-0.29770300$ 
1.67950300

2.37809800

$-2.68208300$

1.40009900

$-0.68794500$

$-0.56792500$

$-0.07340900$

$-1.61026000$

0.11885400

1.18363300

$-0.33367000$

0.04616300

$-4.45010100$

$-4.04243200$

$-3.89192000$

$-5.68594600$

$-6.53186300$

$-5.55646700$

$-5.90001600$

$-3.43002500$

$-3.63445900$

$-2.57742400$

$-4.35787500$

$-5.17852300$

$-3.81919300$

$-4.78093300$

0.81114700

$-0.46029500$

1.08901900

$-1.44962100$

$-0.69247100$

0.13529400

2.06938000

$-1.12142700$

$-2.73837800$

0.37474400

$-1.87556600$
$-1.42033500$

$-1.03378700$

$-1.11784900$

0.99723700

0.32182600

0.49679000

$-1.11256400$

1.27000400

$-1.46529900$

$-0.71748300$

$-0.25601400$

2.46173700

1. 66510100

1.10183700

2.24367800

3.79752600

3. 63051000

4. 34822500

0.78999700

4.38785200

$-0.95527200$

$-1.33353800$

1. 60505700

0.43087500

2. 67457100

$-1.05850100$

$-0.92826500$

1.73031100

$-2.19277300$

1.98905900

$-1.54579200$

2. 54009200

$-2.91503000$

0.78709800

$-2.69732500$

$-2.49103500$

0.49212500

1.14986700

$-0.41270900$

0.84276800

0.11589000

0.76668600

1.84148800

$-0.15543400$

2.73558800

0.35987500

2.37134100

$-1.48462700$

1.80838800

$-0.41173600$

2.72380400

1.38679300

1.72295400

$-2.26923700$

2.58533700

$-1.88967500$

1.43899900

$-1.74053800$

2.30232300

$-0.44420200$

1. 44833100

$-3.29314200$

0.96446500

$-2.36358600$

\section{p-TS4-cf1}

$\mathrm{Pd}$
$\mathrm{C}$
$\mathrm{C}$
$\mathrm{C}$

$-2.68898700$

0.47083400

0.43557700

$-0.83416200$

2. 89322100

0.45657300

0.49310700

3.27604200

0.28623300

1.25686600

2. 76428800

$-0.77285900$ 


\begin{tabular}{|c|c|c|c|}
\hline $\mathrm{C}$ & 0.65341400 & 1.86789200 & -1.66946900 \\
\hline $\mathrm{C}$ & -0.67189200 & 1.48326900 & -1.49812000 \\
\hline $\mathrm{C}$ & -1.44865800 & 1.97596700 & -0.42216400 \\
\hline $\mathrm{H}$ & 0.95931900 & 3.96050000 & 0.98915100 \\
\hline $\mathrm{H}$ & 1.22680900 & 1.47195500 & -2.50495700 \\
\hline $\mathrm{H}$ & -1.13034900 & 0.80868900 & -2.21904700 \\
\hline $\mathrm{C}$ & -4.17791600 & 1.30606000 & -1.88916700 \\
\hline 0 & -3.72047200 & 2.47036200 & -1.65530500 \\
\hline 0 & -3.81258100 & 0.25229800 & -1.28066600 \\
\hline $\mathrm{C}$ & -5.24328100 & 1.16134100 & -2.95047300 \\
\hline 0 & -2.76427800 & -2.71590600 & -0.07463500 \\
\hline $\mathrm{C}$ & -3.64224900 & -2.30947000 & 0.73298600 \\
\hline 0 & -3.75186200 & -1.14112900 & 1.23361000 \\
\hline $\mathrm{C}$ & -4.72133800 & -3.29774900 & 1.14949000 \\
\hline $\mathrm{H}$ & -5.28726600 & 0.13155300 & -3.30867800 \\
\hline $\mathrm{H}$ & -6.21064900 & 1.42035200 & -2.50475600 \\
\hline $\mathrm{H}$ & -5.05223700 & 1.85522300 & -3.77212900 \\
\hline $\mathrm{H}$ & -5.51831500 & -3.27529800 & 0.39674300 \\
\hline $\mathrm{H}$ & -4.31214800 & -4.31017900 & 1.18262100 \\
\hline $\mathrm{H}$ & -5.15069200 & -3.02133600 & 2.11465800 \\
\hline 0 & -0.08054400 & -0.69190100 & 1.75409000 \\
\hline $\mathrm{C}$ & -0.54937800 & 0.28147500 & 2.41466800 \\
\hline 0 & -1.68740800 & 0.80415900 & 2.20918500 \\
\hline $\mathrm{C}$ & 0.31260300 & 0.89156500 & 3.49961600 \\
\hline $\mathrm{H}$ & -0.23290300 & 1.65216500 & 4.05974400 \\
\hline $\mathrm{H}$ & 0.67005900 & 0.10760300 & 4.17419200 \\
\hline $\mathrm{H}$ & 1.18994800 & 1.34578100 & 3.02012600 \\
\hline Ag & -0.82255000 & -1.67172500 & -0.37442000 \\
\hline $\mathrm{C}$ & 3.83346700 & -1.34883100 & -0.65316900 \\
\hline $\mathrm{C}$ & 6.44002400 & -1.53112800 & -1.66789300 \\
\hline C & 4.85352600 & -0.52081300 & -0.13138000 \\
\hline $\mathrm{C}$ & 4.12980600 & -2.22377600 & -1.70607900 \\
\hline $\mathrm{C}$ & 5.42619000 & -2.32671000 & -2.20558400 \\
\hline $\mathrm{C}$ & 6.14805200 & -0.62624300 & -0.64663100 \\
\hline $\mathrm{H}$ & 3.32593000 & -2.82733700 & -2.11479900 \\
\hline $\mathrm{H}$ & 5.64352200 & -3.02224800 & -3.01070600 \\
\hline $\mathrm{H}$ & 6.91801300 & 0.02536800 & -0.24405500 \\
\hline $\mathrm{H}$ & 7.45315400 & -1.60053800 & -2.05377500 \\
\hline $\mathrm{C}$ & 2.42456300 & -1.32719600 & -0.14696800 \\
\hline O & 1.47762500 & -1.58041400 & -0.90549000 \\
\hline 0 & 2.32142900 & -1.05720700 & 1.12907100 \\
\hline $\mathrm{H}$ & 1.34641300 & -0.92993300 & 1.44541400 \\
\hline $\mathrm{C}$ & 2.70270400 & 3.16710500 & -0.96270700 \\
\hline $\mathrm{H}$ & 2.83250700 & 3.57019400 & -1.97562900 \\
\hline
\end{tabular}




\section{p-TS4-cf2}

C

$\mathrm{H}$

$\mathrm{H}$

C

$\mathrm{H}$

$\mathrm{H}$

C

O

$\mathrm{N}$

C

C

C

C

C

$\mathrm{H}$

C

$\mathrm{H}$

$\mathrm{H}$

$\mathrm{H}$

C

$\mathrm{H}$

$\mathrm{H}$

$\mathrm{H}$

C

O

$O$

$\mathrm{Pd}$

$\mathrm{H}$
2.96795800

3.67658500

4.67605500

3.36390600

3. 82814800

3. 37118800

4.60288500

4.90563800

4.82563000

4.19954100

5.92345500

$-1.39825500$

$-2.69605000$
3.95815300

1. 99282700

2.27190100

1.15085400

1.56635400

2.23722900

0.44560200

0.04873600

0.93143500

$-0.71146100$

$-0.35093300$

3.27893000

2.29681500
$-0.25649800$

$-0.79231400$

$-1.15298800$

$-1.41600500$

0.67137300

1.59163900

0.89423400

2. 27102600

2. 90559800

2. 61834000

2.31347300

1.30021700

$-0.89859400$
2.38099800

2.63118900

2.44333800

3. 44783100

3. 31850000

4.44791800

3.43100500

2.84839400

4.14563400

4.84621600

4.21029800

6.23513100

4.99425000

7.00248400

6.70069600

6.37807900

4.50012500

8.08063100

6.96454500

4.03480300

3. 36174100

3. 62726700

5.02218200

2.72418700

1.99467000

2.29703400

$-2.82363700$

1.27660000
3.15883200

3. 41443500

4.07681200

2.17450900

1. 20005600

2.52436900

2.03941900

2.85246600

0.99582500

0.02250600

$-1.06621200$

0.15588100

$-1.98586000$

$-0.77308600$

1.00522600

$-1.85058700$

$-2.81165200$

$-0.65237100$

$-2.58008300$

0.71506000

$-0.13532200$

1. 59928500

0.48323800

$-1.24584600$

$-0.66467100$

$-2.07383500$

$-0.14918300$

$-2.13440700$
1.23021000

2.26627200

0.63776100

0.71112000

1.18984700

1.00254500

$-0.81448100$

$-1.52641600$

$-1.35537700$

$-0.57840000$

0.05779700

$-0.48630300$

0.77347800

0.21499000

$-0.97736100$

0.84525500

1.27144900

0.27156000

1.39593400

$-2.78753400$

$-2.94677800$

$-3.27632400$

$-3.19873700$

0.03267400

$-0.77778100$

0.96521900

0.52643900

0.97877600 


\begin{tabular}{|c|c|c|c|}
\hline Ag & -0.40332900 & -0.67345900 & -1.05178000 \\
\hline $\mathrm{C}$ & -0.97585800 & -1.89979300 & 2.03225700 \\
\hline 0 & -0.24806200 & -2.00848000 & 1.00661600 \\
\hline O & -2.05074200 & -1.22911600 & 2.09451300 \\
\hline $\mathrm{C}$ & -0.55712700 & -2.62632500 & 3.29661600 \\
\hline $\mathrm{H}$ & 0.49215000 & -2.39921200 & 3.51263800 \\
\hline $\mathrm{H}$ & -1.18591600 & -2.34670700 & 4.14293700 \\
\hline $\mathrm{H}$ & -0.63294300 & -3.70568700 & 3.12603100 \\
\hline $\mathrm{C}$ & -4.42564400 & 2.04320500 & -0.36489300 \\
\hline 0 & -3.92266000 & 0.96848700 & -0.82297500 \\
\hline O & -4.08301200 & 2.58905800 & 0.73337700 \\
\hline C & -5.50903300 & 2.70991800 & -1.17906900 \\
\hline $\mathrm{H}$ & -6.47399300 & 2.51297600 & -0.69835900 \\
\hline $\mathrm{H}$ & -5.35420000 & 3.79171700 & -1.18786400 \\
\hline $\mathrm{H}$ & -5.52782500 & 2.30998900 & -2.19376100 \\
\hline C & -3.14152300 & -2.23424700 & -1.47305700 \\
\hline 0 & -3.61478100 & -1.87089800 & -0.33986700 \\
\hline 0 & -2.12945900 & -1.77371700 & -2.06042100 \\
\hline C & -3.93402700 & -3.32509800 & -2.17880900 \\
\hline $\mathrm{H}$ & -4.62687000 & -2.84879900 & -2.88251300 \\
\hline $\mathrm{H}$ & -3.25872300 & -3.96722800 & -2.74948200 \\
\hline $\mathrm{H}$ & -4.51527000 & -3.91228100 & -1.46480100 \\
\hline $\mathrm{C}$ & 0.96849500 & 2.61122900 & 1.17800000 \\
\hline $\mathrm{C}$ & 0.36659800 & 2.05457400 & 2.31802500 \\
\hline $\mathrm{C}$ & 0.22739000 & 2.65272500 & -0.01510600 \\
\hline $\mathrm{C}$ & -0.92879200 & 1.53716300 & 2.27497800 \\
\hline $\mathrm{H}$ & 0.92292300 & 2.02889200 & 3.25329600 \\
\hline $\mathrm{C}$ & -1.06461400 & 2.13701300 & -0.05883200 \\
\hline $\mathrm{H}$ & 0.67804900 & 3.08633900 & -0.89994500 \\
\hline $\mathrm{C}$ & -1.67950300 & 1.56242700 & 1.08379500 \\
\hline $\mathrm{H}$ & -1.36061100 & 1.09113500 & 3.16598700 \\
\hline . & -1.63162300 & 2.21003200 & -0.98437600 \\
\hline & -2.99644900 & 1.98468900 & 1.09597800 \\
\hline
\end{tabular}

\section{p-TS4-cf3}

$\mathrm{Pd}$
$\mathrm{C}$
$\mathrm{C}$
$\mathrm{C}$
$\mathrm{C}$
$\mathrm{C}$
$\mathrm{C}$
$\mathrm{H}$

2.76637500

0.46184200

$-0.19895000$

1.15715500

2.98056000

$-0.78705800$

$-0.12176000$

3. 41451800

$-1.11502200$

$-1.25591400$

2.90000300

$-0.45875900$

$-1.05611000$

1.97122200

0.57114100

0.22650000

1.54936700

0.91150100

1.36811000

2.02046600

0.22894000

$-0.25563400$

4.15619800

$-1.90037400$ 
H

H

C

O

O

C

O

C

O

C

$\mathrm{H}$

$\mathrm{H}$

$\mathrm{H}$

H

$\mathrm{H}$

$\mathrm{H}$

O

C

O

C

$\mathrm{H}$

H

$\mathrm{H}$

Ag

C

C

C

C

C

C

$\mathrm{H}$

H

$\mathrm{H}$

$\mathrm{H}$

C

O

O

$\mathrm{H}$

C

$\mathrm{H}$

$\mathrm{H}$

C

$\mathrm{H}$

$\mathrm{H}$

$\begin{array}{rrr}-1.90403700 & 1.54608700 & 1.08993700 \\ 0.34851200 & 0.83447600 & 1.72328100 \\ 3.60634200 & 1.35803700 & 2.43518300 \\ 3.11845200 & 2.49421300 & 2.13231400 \\ 3.50020200 & 0.31379400 & 1.72294800 \\ 4.38897200 & 1.24499500 & 3.72288800 \\ 2.80206600 & -2.78957000 & 0.12863300 \\ 3.86449100 & -2.29977400 & -0.34066500 \\ 4.07124200 & -1.09197400 & -0.68916600 \\ 5.05416500 & -3.23625600 & -0.48937700 \\ 4.42493300 & 0.20677400 & 4.05687100 \\ 5.41263700 & 1.58896200 & 3.53471600 \\ 3.94997900 & 1.88866000 & 4.48835000 \\ 5.53462500 & -3.34132200 & 0.49048000 \\ 4.71327800 & -4.22534800 & -0.80522500 \\ 5.78326600 & -2.83476100 & -1.19561200 \\ 0.18760000 & -0.34896700 & -1.82609200 \\ 1.00167900 & 0.31665000 & -2.52372500 \\ 2.18195000 & 0.63170700 & -2.16596300 \\ 0.55611600 & 0.81945000 & -3.88192800 \\ 1.40846600 & 1.12991400 & -4.48833300 \\ -0.02519600 & 0.04965800 & -4.39572200 \\ -0.09767100 & 1.68467700 & -3.71858600 \\ 0.83246400 & -1.78905200 & 0.03780800 \\ -3.84253100 & -1.57120000 & 0.24770500 \\ -6.49182500 & -2.28266900 & 0.80621400 \\ -4.76511000 & -0.58873300 & 0.64263900 \\ -4.24937700 & -2.90909200 & 0.17182500 \\ -5.56967500 & -3.26479800 & 0.43979800 \\ -6.08356700 & -0.95397400 & 0.91992100 \\ -3.51991700 & -3.66226400 & -0.10952000 \\ -5.87629900 & -4.30377300 & 0.36332200 \\ -6.78426600 & -0.18270300 & 1.22608800 \\ -7.52411600 & -2.55016600 & 1.01296600 \\ -2.42397300 & -1.26952700 & -0.12250000 \\ -1.49211800 & -1.89956100 & 0.39098800 \\ -2.29833400 & -0.35443300 & -1.05411400 \\ -1.34293300 & -0.26729000 & -1.36838800 \\ -2.62960800 & 3.36325800 & -0.90657100 \\ -2.58191900 & 4.44368700 & -1.09105500 \\ -2.87066300 & 2.89920900 & -1.87206800 \\ -3.80762500 & 3.07948100 & 0.04845700 \\ -4.63465300 & 3.74154000 & -0.22923500 \\ -3.52545200 & 3.32974000 & 1.07291000\end{array}$




\section{p-TS4-cf4}

C

$\mathrm{H}$

$\mathrm{H}$

C

$\mathrm{H}$

$\mathrm{H}$

C

O

$\mathrm{N}$

C

C

C

C

C

$\mathrm{H}$

C

$\mathrm{H}$

$\mathrm{H}$

$\mathrm{H}$

C

$\mathrm{H}$

H

$\mathrm{H}$

C

O

0

$\mathrm{Pd}$

$\mathrm{H}$

Ag

C

o

o
(

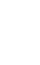

.

C

$\mathrm{N}$

C

(

$-4.35028200$

$-4.75521500$

$-4.33908800$

$-4.12920100$

$-3.34032700$

$-3.83900100$

$-5.04752100$

2.01037400

2. 36950200
1.66039300

1.32642300

0.76047400

1.04869600

0.40353600

2.08663500

0.86248400

3.36867400

2. 30459600
$-0.14877600$

$-1.25222700$

0.90478800

2. 32430700

2.72963100

2.47574700

2.89452200

$-1.33637300$

1.11749000

2.48341100

2.43535800

2.84904800

3.51744400

3. 09248100

4.38222000

4.00582800

3.69708800

4.82344300

5.00757000

3.92789800

6.28941900

4.16045300

6.51508900

7.10402300

5.44630000

3.31547300

7.52074400

5.61118500

5.15285500

6.01652200

5.42520300

4.31628900

2. 52716700

1.56745600

2.42484800

$-2.78705300$

1.46338100

$-0.73601000$

$-0.81307600$

$-0.04363500$

$-2.02762800$

3.64162700
4.65198800
3.73045600
2.83529000
2.53113100
3.47364700
1.59731600
1.37935900
0.72530500
-0.57828400
-1.46710900
-0.96179900
-2.70721900

$-2.21626800$

$-0.25522900$

$-3.08893900$

$-3.36903400$

$-2.50065700$

$-4.06028300$

0.88087300

0.25104000

1.91538300

0.57530700

$-1.17551000$

$-1.71074600$

$-0.37112400$

0.38941200

$-0.25367300$

$-1.68261300$

0.49316100

$-0.19818500$

0.75005600
$-0.41712700$

0.00512700

$-1.44451300$

0.39475400

1.35918100

0.62266600

$-0.35414500$

$-1.52095900$

0.33679100

$-0.23314500$

$-0.41808100$

$-0.62560800$

$-1.02571700$

$-1.19469800$

$-0.49733200$

$-1.40278700$

$-1.18393500$

$-1.49139900$

$-1.85944200$

1.75341100

1.97874300

1.97428600

2. 39330400

0.01113100

$-0.55253900$

1.05314300

0.31729000

1.35129900

$-0.07995900$

2.46768000

1.74568500

2.19641900 


$$
\begin{array}{r}
-0.26434200 \\
0.29502400 \\
-1.06913200 \\
0.42586700 \\
-4.01975100 \\
-3.69705800 \\
-3.64418400 \\
-4.92905200 \\
-5.95260800 \\
-4.64019200 \\
-4.90375500 \\
-3.64058600 \\
-3.88817900 \\
-2.61118200 \\
-4.73906900 \\
-5.41335900 \\
-4.31043300 \\
-5.31953300 \\
1.08979900 \\
0.12566100 \\
0.72345700 \\
-1.16227100 \\
0.39055900 \\
-0.56787500 \\
1.46048500 \\
-1.54359000 \\
-1.88460400 \\
-0.84254500 \\
-2.71153800
\end{array}
$$

\section{p-TS4-cf5}

$\mathrm{Pd}$
$\mathrm{C}$
$\mathrm{C}$
$\mathrm{C}$
$\mathrm{C}$
$\mathrm{C}$
$\mathrm{C}$
$\mathrm{H}$
$\mathrm{H}$
$\mathrm{H}$
$\mathrm{C}$
$\mathrm{O}$

$-2.62827900$

0.26089200

0.08106000

$-1.48360700$

$-1.78897200$

1.97440500

$-0.22142300$

$-2.26195800$

2. 34196600

0.81172400

$-1.37691700$

2.68462100

0.52555700

$-0.00581200$

2.72293900

0.47099600

2. 31766500

$-0.40414400$

1.90604500

2.30896500

$-0.01582100$

$-3.32857600$

3. 01076100

0.70017600

2.28288100

$-0.87656000$

1.54363000

1.98196400

$-3.70064600$

2.73158100 
$-2.87982400$

$-4.09661000$

$-0.69794800$

$-1.85378700$

$-2.91078400$

$-2.01268000$

$-3.69963600$

$-5.18488200$

$-3.88598300$

$-1.58106200$

$-1.44758800$

$-3.06089000$

$-1.33187100$

$-2.50465600$

$-3.06086800$

$-3.40059600$

$-4.06960800$

$-4.01956400$

$-2.79820900$

0.02354100

3.73083600

6.43397500

4.47776300

4.35653200

5.70181500

5.82621300

3.77370500

6.17575800

6.39306600

7.48289700

2.26985600

1.60142500

1.78510800

0.78958400

2.22161300

2.50291900

2.26635300

3. 27257700

4.28213200

3.23083700

3.10844100

2. 34635000

3.91230800

3.75794100
2.16841400

0.88223800

3.50474400

2.43859200

1.47036500

$-1.83949300$

1.59634700

1.08321800

$-2.33702800$

2.38931800

4.32810600

3.46370600

3.65454300

3.38666000

1.89184700

2.47384700

$-2.31739400$

$-2.39892100$

$-1.64186100$

$-3.50106600$

$-3.86948600$

$-3.07979100$

$-4.31418800$

$-0.89789300$

1.44269600

1.63724100

0.26932000

2. 69228800

2. 79255000

0.38225200

3.58029200

3.76797400

$-0.52689900$

1.70842000

1. 34497000

0.49447400

2.20249400

2.03241700

$-1.86193600$

$-1.62067800$

$-2.94964900$

$-1.22529500$

$-1.50732700$

$-0.13331600$

$-1.66275400$

$-2.56906700$

$-1.03583800$

$-1.44599200$
$-1.84615400$

$-3.62247400$

1.84341000

2.31744900

3. 50081400

$-3.48723800$

$-4.41852600$

$-3.91285800$

$-1.89911700$

$-1.46077600$

$-0.59465900$

$-2.00321600$

$-1.22186700$

$-2.80385500$

$-2.41273500$

$-0.90987100$

$-0.42640800$

0.26513200

$-0.16457600$

$-0.36796000$

$-0.01279400$

0.17322900

$-0.59095600$

0.04399200

0.34910400

0.53851300

$-0.69772900$

$-0.10253000$

$-1.56869300$

$-1.71283700$

2. 95457600

3. 98867500

2. 85011000

2.02373700

2.35196000

2.07629000

0.57280200

0.24755700

$-0.36442100$

$-1.76359500$ 
$\mathrm{H}$

$\mathrm{H}$

$\mathrm{H}$

$\mathrm{H}$

$\mathrm{H}$

\section{$p$-TS4-cf6}

3.58361600

2.90579900

4.67148300

$-2.24873300$

$-2.94979700$
$-2.52130700$

$-0.94501100$

$-1.19527000$

$-2.49148000$

0.24793900
$-1.79778400$

$-2.24458100$

$-2.30816100$

1. 66021800

2.28185900
C

$\mathrm{H}$

$\mathrm{H}$

C

$\mathrm{H}$

$\mathrm{H}$

C

O

$\mathrm{N}$

C

C

C

C

C

$\mathrm{H}$

C

$\mathrm{H}$

$\mathrm{H}$

$\mathrm{H}$

C

$\mathrm{H}$

$\mathrm{H}$

$\mathrm{H}$

C

O

$\mathrm{O}$

$\mathrm{Pd}$

$\mathrm{H}$

Ag

C

O

O

C

$\mathrm{H}$

$\mathrm{H}$

$\mathrm{H}$

(

(1)

C

C

(n)

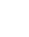

(n)

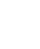

(1)

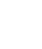

(

(

(

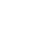

(1)

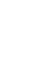

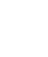

(

$\mathrm{Pd}$

Ag

O

C

$\mathrm{H}$

$\mathrm{H}$

$\mathrm{H}$

$$
\begin{aligned}
& -1.78275500 \\
& -2.02253700 \\
& -1.95382500 \\
& -2.74656600 \\
& -2.36609800 \\
& -3.72054900 \\
& -2.97692300 \\
& -2.43306000 \\
& -3.88873600 \\
& -4.34358500 \\
& -3.67336200 \\
& -5.52634200 \\
& -4.21397000 \\
& -6.05041600 \\
& -6.02746200 \\
& -5.39079600 \\
& -3.69093800 \\
& -6.96955000 \\
& -5.78936000 \\
& -4.19814900 \\
& -5.24299300 \\
& -3.54318800 \\
& -4.04506400 \\
& -2.38537000 \\
& -1.88678400 \\
& -1.82175700 \\
& 2.61005400 \\
& -0.88040000 \\
& 0.26609600 \\
& 0.03007100 \\
& -0.66095900 \\
& -0.30962100 \\
& -1.58127700 \\
& -0.96159100 \\
& -0.02117800
\end{aligned}
$$

$-3.38313400$

1.01589200

2.01984700

0.30819600

0.67938000

1.09042200

1.15238100

$-0.82606100$

$-1.64972100$

$-1.21046800$

$-0.33034000$

$-0.17787400$

0.37934400

0.69237700

1.24013000

0.24124600

1.39563000

1.88314900

0.81201900

.06764500

1.78316700

2.64224200

2.06234200

$-2.63358200$

$-2.73582600$

$-0.64260200$

$-0.20617500$

$-3.09506500$

$-3.12341900$

$-1.91249700$

$-0.87289400$

0.70223800

$-1.70807900$

2.60376700

$-0.51161600$

0.45471300

0.51464600

2.63223400

$-0.87127100$

$-0.11513000$

$-1.08193200$

0.93900900

1.97031100

0.35045200

1.11848100

1.07894300

1.96651100

1.54216800

3.18019400

2.04474900

2. 84773600

0.72861500

3.85432500

2.23623500

3.71878400 


\section{p-TS4-cf7}

$\mathrm{Pd}$
$\mathrm{C}$
$\mathrm{C}$
$\mathrm{C}$
$\mathrm{C}$
$\mathrm{C}$
$\mathrm{C}$
$\mathrm{H}$
$\mathrm{H}$
$\mathrm{H}$
$\mathrm{C}$
$\mathrm{O}$
$\mathrm{O}$
$\mathrm{C}$
$\mathrm{O}$
$\mathrm{C}$

Pd

$\begin{array}{rrr}4.91772900 & -0.94036600 & -0.56028400 \\ 4.11658400 & 0.00198300 & -0.82960100 \\ 4.74177100 & -1.80188400 & 0.36866100 \\ 6.17955300 & -1.05434600 & -1.38061200 \\ 7.01881500 & -0.69049400 & -0.77699000 \\ 6.37272600 & -2.10207800 & -1.62422200 \\ 6.10271700 & -0.45030400 & -2.28556700 \\ 1.71227100 & 2.97619400 & -0.65681100 \\ 2.71444200 & 2.47705300 & -0.05824600 \\ 0.73353500 & 2.32875400 & -1.13581500 \\ 1.69367300 & 4.48930100 & -0.79855700 \\ 1.50407700 & 4.75740800 & -1.84232500 \\ 0.86507800 & 4.88592000 & -0.20000000 \\ 2.62982900 & 4.93307600 & -0.45677200 \\ -0.34134900 & -2.93317300 & 0.98095500 \\ 0.28910000 & -2.49939000 & 2.15330600 \\ 0.36059000 & -2.83055500 & -0.23599800 \\ 1.51814400 & -1.84128900 & 2.11494100 \\ -0.22668100 & -2.61621100 & 3.10426500 \\ 1.59979600 & -2.17662900 & -0.27393000 \\ -0.08864100 & -3.23127900 & -1.13956200 \\ 2.17887600 & -1.60355600 & 0.89744400 \\ 1.93255800 & -1.43385700 & 3.03267400 \\ 2.15813500 & -2.14490800 & -1.21011400 \\ 3.60257000 & -1.65299500 & 0.78170100\end{array}$

4.91772900

$-0.94036600$

$-0.82960100$

0.36866100

$-1.38061200$

99000

$-1.62422200$

$-0.65681100$

$-0.05824600$

$-1.13581500$

$-0.79855700$

$-0.20000000$

$-0.45677200$

2.15330600

$-0.23599800$

.11494100

$-0.27393000$

200

0.78170100

2.81300600

0.34259500

0.15720400

2.15380200

$-2.49080000$

1.10807800

0.95326400

$-3.16872800$

1.31793700

$-0.09169100$

$-3.11129100$

0.37847500

0.14287800

$-2.43691100$

$-0.83078700$

1. 35162600

$-1.77099900$

$-1.04706600$

2. 36953600

$-1.73739400$

$-0.06430900$

0.79038300

$-3.69656000$

2.25538400

$-0.62751100$

$-2.37306000$

$-1.59159800$

1. 48275400

$-1.21865100$

$-1.97382800$

4.25722000

$-0.41499100$

$-2.22318400$

4.47641200

$-1.52281700$

$-1.62239600$

3.46226800

0.48222200

$-1.81481100$

5.01224000

$-0.16259000$

$-3.50639400$

0.62324600

2.15196700

$-0.82385700$

1.52023500

2. 87395000

$-0.29872300$ 
2.59550300

1.31411800

4.57375000

6.05223300

5.01600400

1.14334100

0.41290500

2.17121800

0.42002900

1.58866000

2.61544500

1.88970400

2.30668100

0.97329300

2.63766900

$-0.27807700$

$-3.89470900$

$-6.53117500$

$-4.56429300$

$-4.56910500$

$-5.87666000$

$-5.87095000$

$-4.04520700$

$-6.37659900$

$-6.36067800$

$-7.54674200$

$-2.47740800$

$-1.85760400$

$-1.94028200$

$-0.92702100$

$-1.43654800$

$-1.35914700$

$-1.66570500$

$-2.61680100$

$-3.48660300$

$-2.40235200$

$-3.04192400$

$-2.58105100$

$-4.01752600$

$-4.35579600$

$-3.55865400$

$-4.53373700$

$-5.27526300$

2. 90204600
2.43896300

4.37878600

0.67573500

0.07569500

$-1.06675100$

4.73557900

4.60163500

4.89393800

0.74686200

0.57625200

0.24807900

0.78614600

1.79164800

0.70384800

0.06828900

0.08258900

1.22291600

1.23798800

0.00756600

2.42900500

2. 44315900

0.03256900

3. 35949600

3.39014600

$-0.91489400$

1.23280600

1.27008100

0.27404200

2.48054200

2.43074200

$-3.73532800$

$-4.82668700$

$-3.53805200$

$-3.26244800$

$-3.90035600$

$-3.40296900$

$-1.81582700$

$-1.18361600$

$-1.29795300$

$-1.87895900$

$-1.68487100$

$-2.95422800$

$-1.41169500$

$-2.47408600$
0.22699000

$-0.27675200$

$-4.04927800$

$-3.25600800$

$-4.12057700$

$-1.29784700$

0.30567800

0.15932400

2. 47521100

2. 88116200

2.18425300

4.35778000

4.48690400

4.94528100

4.70385200

0.42637900

$-0.67811800$

0.30548400

$-0.42123900$

$-0.42670000$

0.04986200

0.07270300

$-0.60948600$

0.23052900

0.27714200

0.69114000

$-1.14896100$

$-1.53114300$

$-1.07313400$

$-1.15450100$

0.70105100

0.59576200

1.75343000

$-0.15702100$

0.05268200

$-1.22107000$

0.12502900

1.07739500

$-0.68693500$

$-1.98958200$

$-2.71802200$

$-1.91318200$

$-2.34620000$

1.89472900 


\section{p-TS4-cf8}

C

$\mathrm{H}$

$\mathrm{H}$

C

$\mathrm{H}$

$\mathrm{H}$

C

$\mathrm{O}$

$\mathrm{N}$

C

C

C

C

C

$\mathrm{H}$

C

$\mathrm{H}$

$\mathrm{H}$

$\mathrm{H}$

C

$\mathrm{H}$

$\mathrm{H}$

$\mathrm{H}$

C

O

$\mathrm{O}$

$\mathrm{Pd}$

$\mathrm{H}$

Ag

C

O

O

C

$\mathrm{H}$

$\mathrm{H}$

$\mathrm{H}$

C

O

O

C
1.74481200

1.53128800

2.28050800

2.68344600

2.10652900

3.40021800

3.47612400

3.47686500

4.22436300

4.72991900

3.93889400

6.06889000

4.52839000

6.64173900

6.65172800

5.86847900

3.91893400

7. 68679000

6.30466800

4.21600600

5.10254000

4.27084800

3. 32080700

2. 50130500

1. 95726200

1.87906600

$-2.74357100$

0.91648200

$-0.18445200$

$-2.70084700$

$-1.46178200$

$-3.34290400$

$-3.56383900$

$-3.61561600$

$-4.57845200$

$-3.11092700$

$-3.28510800$

$-2.71939700$

$-3.57896600$

$-3.64059300$
$-3.37152200$

1.62271300

$-4.43694100$

1.48270800

$-3.26047100$

2.57134000

$-2.91277000$

$-2.74538500$

0.48245400

$-0.43533600$

$-3.71467500$

0.25665000

$-1.64735500$

0.82187000

$-1.15273500$

1.94460500

$-1.08811400$

$-0.19310500$

0.23994700

0.02564500

1. 40215900

$-0.14384600$

0.36966000

0.39189800

2. 65966600

0.06101500

1. 62655200

0.58783100

$-0.53556800$

0.52947800

2.77575000

0.42021200

3. 54635700

1.70500100

$-0.06837100$

3.75872100

0.87416700

0.57126900

$-1.56880800$

$-1.17817000$

$-1.57436100$

$-2.08013500$

$-2.65929100$

$-1.59971700$

$-1.23606900$

1.33099700

0.25751100

2. 49462100

0.35143700

$-2.11252700$

$-0.53161400$

$-0.81240700$

$-0.56431200$

0.11876900

2.39586800

$-0.85443600$

$-0.36577500$

$-1.24233600$

$-1.84534300$

$-2.02114100$

$-1.87422900$

$-1.08363300$

$-2.21632200$

$-1.22259300$

$-2.85067200$

$-2.76763400$

$-3.77081400$

$-2.17295000$

$-2.46994500$

$-2.89980800$

$-3.09132300$

$-3.73176300$

1. 52320300

2.67545900

1.82893400

1.57973700

0.33445100

3.03286500

2.64563600

3.62126400 


\section{$o_{1}$-TS4-cf1}

$\mathrm{Pd}$
$\mathrm{C}$
$\mathrm{C}$
$\mathrm{C}$
$\mathrm{C}$
$\mathrm{C}$
$\mathrm{C}$
$\mathrm{H}$
$\mathrm{H}$
$\mathrm{H}$
$\mathrm{H}$
$\mathrm{C}$
$\mathrm{O}$
$\mathrm{O}$
$\mathrm{C}$
$\mathrm{O}$
$\mathrm{C}$
$\mathrm{O}$
$\mathrm{C}$
$\mathrm{H}$

$-4.72602400$

$-3.36910400$

$-3.14458600$

$-1.78269700$

$-2.88312400$

$-0.63593200$

$-1.85597400$

$-1.43031200$

$-1.24418400$

$-2.88503400$

0.44151400

$-0.76275500$

0.42516200

$-1.93473200$

$-0.77244900$

$-0.73601100$

1.33985800

$-1.94279700$

$-2.83825000$

$-0.70735400$

$-2.99059900$
2.79324500

3.59210800

2.36867000

4.64319200

3.57049000

2. 51719400

3. 32399900

1. 92942900

2.06327400

3.86074800

4.62752700

3.82937100

4.12275000

$-2.59958900$

$-3.17021600$

$-1.26587100$

$-2.41513400$

$-4.20939600$

$-0.51076600$

$-0.82293300$

$-1.05189200$

$-2.86686500$

0.53055300

$-0.43903300$
$-1.53812600$

$-1.30092500$

$-1.23889800$

$-2.24164900$

$-1.58484600$

$-3.14922900$

$-2.49148300$

1.66874400

1.22426400

2.11038300

1.14365300

0.90195800

2. 01434700

2.48575200

1.50170300

0.74507700

2.32157000

2. 21498400
1.68875900

1.90764700

1.18183400

0.89878200

1.32869200

2.05804900

2. 35106100

0.07729000

1. 10332100

2.39016000

2.90994300

$-0.49678400$

$-0.90625400$

0.57152200

$-1.31216000$

2. 49584300

1. 78785900

0.60329700

2. 44075800

$-1.47543800$
0.29115000

0.85607500

3.20878800

1.11938200

2. 29557800

0.31840800

2. 64836200

3.88943300

$-1.03138300$

4.76588400

$-1.50955200$

4.43049300

$-0.70186700$

1.93998000

0.62180600

4.17149800

1.01996200

$-2.53548200$

5.71831300

$-1.10782400$

5.11535800

1.25368200

0.92830600

2. 70261400

1. 77028000

1.83939000

0.25607000

2.59396900

0.72845100

3.95793600

$-1.53675800$

1.51418800

$-2.57288000$

1.69829300

$-2.75130400$

1.28061800

$-3.71312700$

2. 46021400

$-0.34115700$

4.10654400 


\begin{tabular}{|c|c|c|c|}
\hline $\mathrm{H}$ & -0.74510300 & 1.10907300 & 4.81412000 \\
\hline $\mathrm{H}$ & -2.26420000 & 1.25528300 & 3.88440400 \\
\hline $\mathrm{H}$ & 3.47436400 & -3.48039300 & 2.72043800 \\
\hline $\mathrm{H}$ & 1.86308500 & -3.90601700 & 3.37086500 \\
\hline $\mathrm{H}$ & 2.39929200 & -4.62119900 & 1.85011800 \\
\hline 0 & 3.02496200 & 0.36650300 & -0.69202500 \\
\hline $\mathrm{C}$ & 3.18656500 & -0.48666200 & -1.63045300 \\
\hline 0 & 2.41443600 & -1.41765500 & -1.96507100 \\
\hline C & 4.48434700 & -0.32181100 & -2.40471300 \\
\hline $\mathrm{H}$ & 4.41403400 & -0.81856100 & -3.37406800 \\
\hline $\mathrm{H}$ & 4.72016500 & 0.73804200 & -2.52824600 \\
\hline $\mathrm{H}$ & 5.29446500 & -0.78067700 & -1.82620300 \\
\hline $\mathrm{Ag}$ & 0.44152900 & -1.85436500 & -1.10251000 \\
\hline $\mathrm{C}$ & -3.36433100 & -0.41970400 & 0.46220800 \\
\hline $\mathrm{C}$ & -5.49654300 & 1.40472400 & 0.49902000 \\
\hline $\mathrm{C}$ & -3.78716800 & 0.19981700 & -0.73899700 \\
\hline $\mathrm{C}$ & -4.01381000 & -0.09749900 & 1.66297600 \\
\hline $\mathrm{C}$ & -5.07821100 & 0.79874500 & 1.68519600 \\
\hline $\mathrm{C}$ & -4.84865400 & 1.10723100 & -0.69966000 \\
\hline $\mathrm{H}$ & -3.67258600 & -0.56485400 & 2.57915900 \\
\hline $\mathrm{H}$ & -5.57404600 & 1.02814700 & 2.62370800 \\
\hline $\mathrm{H}$ & -5.15131200 & 1.57986600 & -1.62907300 \\
\hline $\mathrm{H}$ & -6.31961300 & 2.11363600 & 0.50641600 \\
\hline $\mathrm{C}$ & -2.20640100 & -1.35712400 & 0.48917400 \\
\hline $\mathrm{O}$ & -1.78621300 & -1.92001100 & -0.52866200 \\
\hline $\mathrm{O}$ & -1.65263100 & -1.50632600 & 1.67783900 \\
\hline $\mathrm{H}$ & -0.77657400 & -2.01766800 & 1.59089100 \\
\hline $\mathrm{C}$ & 0.14981600 & 1.71594100 & -1.95630800 \\
\hline $\mathrm{H}$ & 0.06441900 & 2.15926600 & -2.95369100 \\
\hline $\mathrm{H}$ & 0.73096800 & 0.79889700 & -2.08857700 \\
\hline C & -1.25010600 & 1.34546600 & -1.44750600 \\
\hline $\mathrm{H}$ & -1.90596900 & 2.22445900 & -1.41492300 \\
\hline $\mathrm{H}$ & -1.19367100 & 0.99504300 & -0.41431000 \\
\hline $\mathrm{C}$ & -1.88773700 & 0.25774100 & -2.30597200 \\
\hline $\mathrm{O}$ & -1.28026800 & -0.28019900 & -3.22824200 \\
\hline $\mathrm{N}$ & -3.19454400 & -0.07966600 & -2.01271000 \\
\hline $\mathrm{C}$ & -3.79739500 & -1.18036500 & -2.77186000 \\
\hline $\mathrm{H}$ & -3.45930800 & -1.11745400 & -3.80631100 \\
\hline & -3.49040800 & -2.14595500 & -2.35448500 \\
\hline $\mathrm{H}$ & -4.88544000 & -1.08536400 & -2.72772500 \\
\hline $\mathrm{H}$ & 2.10498600 & 2.95284900 & 2.15832700 \\
\hline
\end{tabular}

\section{$o_{1}$-TS4-cf2}




\begin{tabular}{|c|c|c|c|}
\hline $\mathrm{Pd}$ & -2.04213900 & 0.61881800 & -0.40543600 \\
\hline C & -3.21048100 & 0.32214700 & 2.30368700 \\
\hline $\mathrm{C}$ & -2.00958500 & 0.81191500 & 1.73881000 \\
\hline $\mathrm{C}$ & -0.79310300 & 0.61736500 & 2.46278700 \\
\hline C & -0.82325700 & -0.13741400 & 3.64188600 \\
\hline C & -2.01480200 & -0.66582600 & 4.14017900 \\
\hline C & -3.22317100 & -0.40637400 & 3.48880300 \\
\hline $\mathrm{H}$ & -2.06113800 & 2.10891200 & 1.30754900 \\
\hline $\mathrm{H}$ & 0.10073200 & -0.29129600 & 4.19525300 \\
\hline $\mathrm{H}$ & -2.00266100 & -1.25155700 & 5.05591300 \\
\hline $\mathrm{H}$ & -4.16020600 & -0.77255200 & 3.90000100 \\
\hline $\mathrm{C}$ & -1.16451100 & 3.34824400 & -0.07599000 \\
\hline O & -1.84774600 & 3.34836800 & 0.99931000 \\
\hline O & -0.93709700 & 2.31092500 & -0.77404500 \\
\hline C & -0.54224500 & 4.63847400 & -0.54284100 \\
\hline O & -2.03028800 & 0.45021100 & -2.52521100 \\
\hline $\mathrm{C}$ & -0.93884800 & 0.20996700 & -3.11740800 \\
\hline O & 0.03389500 & -0.44125700 & -2.61812100 \\
\hline C & -0.78019500 & 0.74808200 & -4.52610900 \\
\hline $\mathrm{H}$ & -0.70705800 & 4.75819100 & -1.61690900 \\
\hline $\mathrm{H}$ & -0.95267200 & 5.48557500 & 0.00825100 \\
\hline $\mathrm{H}$ & 0.53444400 & 4.55485500 & -0.36757800 \\
\hline $\mathrm{H}$ & -1.74983600 & 0.99598500 & -4.96111400 \\
\hline $\mathrm{H}$ & -0.17589200 & 1.66197400 & -4.47343800 \\
\hline $\mathrm{H}$ & -0.24805800 & 0.02603100 & -5.15086500 \\
\hline O & -3.41772600 & -0.90252900 & -0.20616600 \\
\hline C & -3.22149600 & -2.09590400 & 0.21024900 \\
\hline O & -2.12085000 & -2.69321900 & 0.30790900 \\
\hline $\mathrm{C}$ & -4.47305800 & -2.82384300 & 0.66734600 \\
\hline $\mathrm{H}$ & -4.35097800 & -3.90266100 & 0.55234500 \\
\hline $\mathrm{H}$ & -4.61741400 & -2.60082600 & 1.73145200 \\
\hline $\mathrm{H}$ & -5.34990300 & -2.47038700 & 0.12077100 \\
\hline Ag & -0.41159400 & -1.87612000 & -0.81227100 \\
\hline C & 3.83258100 & -1.12364000 & -0.10468800 \\
\hline $\mathrm{C}$ & 5.95835700 & -1.16738100 & 1.72161300 \\
\hline $\mathrm{C}$ & 4.25610200 & 0.07637900 & 0.50271500 \\
\hline C & 4.45154400 & -2.33261100 & 0.25080300 \\
\hline $\mathrm{C}$ & 5.51883800 & -2.36018700 & 1.14100100 \\
\hline $\mathrm{C}$ & 5.32081300 & 0.03174000 & 1.41535100 \\
\hline $\mathrm{H}$ & 4.07967100 & -3.24693600 & -0.19927900 \\
\hline $\mathrm{H}$ & 5.99904800 & -3.30250200 & 1.38653000 \\
\hline $\mathrm{H}$ & 5.63177100 & 0.95612000 & 1.89284200 \\
\hline $\mathrm{H}$ & 6.78285800 & -1.17265500 & 2.42905300 \\
\hline $\mathrm{C}$ & 2.69054800 & -1.21816100 & -1.06001100 \\
\hline
\end{tabular}


1.96872200

2.51778100

1.64139500

0.49475600

0.25984700

1.11778500

1. 34382900

0.70192700

1. 78780800

2.36970400

2.04108600

3. 67395600

4.54345200

4.08820200

4.67153400

5.51204100

$-4.14446800$
$-2.21863500$

$-0.17496100$

$-0.26619800$

1.33563900

2. 36308400

1.40087400

0.71902100

0.50755000

$-0.22280000$

1.72835200

2.89422200

1. 34809000

2.39439300

2. 79523700

3.21951800

1. 95441500

0.50015200
$-1.06937400$

$-1.85264000$

$-2.34049100$

2.10381900

1.82278900

3.00437100

0.97791500

0.11562000

1.30823700

0.47051600

0.26263500

0.23275900

$-0.32447600$

$-1.23401600$

0.38323600

$-0.56364400$

1.78045100

\section{$o_{1}$-TS4-cf3}

$\mathrm{Pd}$
$\mathrm{C}$
$\mathrm{C}$
$\mathrm{C}$
$\mathrm{C}$
$\mathrm{C}$
$\mathrm{C}$
$\mathrm{H}$
$\mathrm{H}$
$\mathrm{H}$
$\mathrm{H}$
$\mathrm{C}$
$\mathrm{O}$
$\mathrm{O}$
$\mathrm{C}$
$\mathrm{O}$
$\mathrm{C}$
$\mathrm{O}$
$\mathrm{C}$
$\mathrm{H}$
$\mathrm{H}$
$\mathrm{H}$
$\mathrm{H}$
$\mathrm{H}$

0.55118100

$-2.01152800$

$-1.57846800$

$-2.38450300$

$-3.57490400$

$-3.96993800$

$-3.19425400$

$-1.30959500$

$-4.19532200$

$-4.89380900$

$-3.51284000$

0.04847900

$-1.16060300$

0.93709000

0.46888300

2.67779500

3. 39411600

3.04434200

4.78524400

0.93459400

1.22788100

$-0.38280000$

5.07678700

4.77568700
0.85434200

1.61360300

1.03848700

0.03276800

$-0.35726400$

0.21712800

1.21476100

1.98050300

$-1.12375600$

$-0.10886700$

1.66789100

2.70397700

2.79156500

2.01149700

3. 45095600

0.77816300

0.46587600

$-0.30532200$

1.06859100

2. 74208700

4.19237700

3.94711800

1.49203800

1.86807100 
$-0.64117300$

$-3.53820900$

$-3.44541800$

$-2.42198000$

$-4.73392000$

$-4.68607000$

$-4.86241300$

$-5.58949500$

$-0.58379800$

3. 91260600

6.51051600

4.10988900

5.02193800

6.31643100

5.41038100

4.85663300

7.16834800

5.54729100

7.51603400

2.54834500

1.70293700

2.36485200

1.44966700

$-0.06739800$

$-0.27258700$

0.35685100

1.00762800

0.77807300

1.05891300

2.33225500

2.72645600

2.99894300

2.78401800

1.73649700

3.12284900

3.36211500

$-3.65908700$
5.50638300

0.28465200

0.00930100

0.08941700

$-0.50627700$

$-0.45458700$

$-1.55051700$

0.05986800

1.19547000

0.44772400

$-0.59292900$

$-0.94502800$

1. 30320500

0.78726200

$-1.44972800$

2.37440000

1.45997000

$-2.52400000$

$-1.00373600$

1.03914300

0.55424900

2.13798200

2.51516400

$-1.95103800$

$-1.49917700$

$-1.15033400$

$-3.04671100$

$-3.67051000$

$-3.69527300$

$-2.34431100$

$-2.15836500$

$-1.82771200$

$-2.30643500$

$-2.23293800$

$-3.34249200$

$-1.67283800$

$-1.40371500$
0.31434500

$-0.33781200$

$-1.58667300$

$-2.30989400$

$-2.20345400$

$-3.29242000$

$-1.89502700$

$-1.82646500$

$-1.66208700$

$-0.27964000$

$-0.20056200$

$-0.18584400$

$-0.30430000$

$-0.27471200$

$-0.14470000$

$-0.35726900$

$-0.30961600$

$-0.06691800$

$-0.18005800$

$-0.41806100$

$-1.16434600$

0.30574000

0.13088400

$-0.58824000$

$-1.56611900$

0.02038600

$-0.79005800$

$-1.65667600$

0.09020400

$-1.08613200$

$-2.22579400$

0.01585400

1.38713300

1.68411400

1.51974700

2.06218900

2.38642700

\section{$o_{1}$-TS4-cf4}

$\mathrm{Pd}$
$\mathrm{C}$
$\mathrm{C}$
$\mathrm{C}$

1.56382400

1.55291200

1.16971300

1. 35416200
$-0.08494100$

0.81723900

2. 40006700

2.20831900

2.00941600

0.89473700

2. 94148800

$-0.16497900$ 
1.88298500

2.26995800

2. 10353600

$-0.07864300$

2.00093200

2. 68827900

2.38607000

$-0.92357900$

$-1.21719600$

0.19747900

$-1.94012700$

2.16627200

1.28626300

0.24355700

1. 50541600

$-1.83902200$

$-2.94403400$

$-1.76860200$

2. 57048500

1.02779100

1.04204600

3.02498500

3.09989700

2. 25955800

4.37025200

5.10516000

4.16636900

4.79149100

0.54702300

$-3.50807700$

$-5.45208000$

$-3.40199800$

$-4.59427000$

$-5.57171800$

$-4.37159400$

$-4.65802100$

$-6.41409100$

$-4.25186700$

$-6.19858400$

$-2.44339500$

$-1.82640300$

$-2.19930100$

$-1.36554000$

1.06772700
4.20305600

4.54963900

3. 64264600

1.50249800

4. 93200200

5.53520500

3.90916700

0.13758800

1.07596000

$-0.46750900$

$-0.24843700$

$-2.11258200$

$-3.01306800$

$-3.15522400$

$-3.97620000$

0.44187200

$-0.14495900$

$-1.26843800$

$-4.09090200$

$-3.54180500$

$-4.94325100$

0.28509600

$-0.19889500$

$-0.92730600$

0.16047900

$-0.63625000$

0.21908700

1.09790700

$-1.88630300$

$-0.48816800$

1. 51199100

0.68789900

$-0.64486300$

0.34205800

1.68342000

$-1.54692100$

0.20500700

2.59983000

2.29634100

$-1.52601700$

$-1.82196700$

$-2.09361800$

$-2.64759400$

2.71018800
0.14499100

1. 43683600

2.48662800

1.00731700

$-0.65385200$

1.62580000

3. 50122800

2.31290600

1. 51390400

2. 32312900

3. 35958700

1.21236200

1. 38044300

0.66773200

2.53021400

4.20548300

2.94557800

3. 70416200

2.74200000

3.41738300

2. 32297700

$-0.57488000$

$-1.75513200$

$-2.34254200$

$-2.50905200$

$-2.34464600$

$-3.58071100$

$-2.14022800$

$-1.30822000$

$-0.19144600$

0.02838500

$-0.97183200$

0.68062800

0.78143500

$-0.83660500$

1.28066400

1.45316400

$-1.40227300$

0.11682400

$-0.22718300$

$-1.25178100$

0.95058600

0.88665600

$-1.63934100$ 
$\mathrm{H}$

$\mathrm{H}$

C

$\mathrm{H}$

$\mathrm{H}$

C

O

$\mathrm{N}$

C

$\mathrm{H}$

$\mathrm{H}$

$\mathrm{H}$

$\mathrm{H}$
0.77595400

2.02129800

0.04392200

0.37519000

0.00564900

$-1.34670100$

$-1.59408100$

$-2.27260700$

$-2.21330500$

$-2.34826100$

$-1.27293900$

$-3.01447200$

1.38132000
3.67481600

2.43994800

1.66523800

0.66187800

1.65627300

1.87645000

2.78616800

0.89223800

0.19128500

0.89808700

$-0.34767800$

$-0.54495000$

1.70498300
$-2.06977100$

$-2.11389800$

$-2.07218400$

$-1.78675800$

$-3.16813900$

$-1.48653300$

$-0.70991700$

$-1.82154600$

$-3.11013600$

$-3.93958000$

$-3.24299200$

$-3.14111000$

3.02604600

\section{$o_{1}$-TS4-cf5}

Pd
C
C
C
C
C
C
H
H
H
H
C
O
O
C
O
C
O
C
H
H
H
H
H
H
O
C
O

2.22157600

0.69973100

$-0.28812700$

1. 96542400

3.10274700

1.37952900

1.07447600

2. 19186500

0.75824300

$-0.20215000$

2.66366800

0.35017000

$-0.53781400$

4.00279700

0.58747300

0.36167600

4.88042600

1.19139000

1. 62303600

4.43281800

1.59402400

1.00167400

1.04704900

1.53831500

$-1.50861400$

4.37847000

0.27513700

0.07554900

5.91740600

1.34918300

2. 32309600

5.11325700

2. 07118400

2.41448000

$-0.10074300$

2.44548100

1.16564500

0.16680300

2.47539500

3. 18295700

0.22905400

1. 49092300

3. 01883000

$-0.88839800$

3.57956900

3. 58828400

$-0.57545300$

$-1.24512000$

3.83260600

$-1.75241000$

$-0.81976500$

3.08819600

$-2.46728600$

$-0.09240300$

5.18206400

$-2.32198200$

$-1.22607100$

3.20679600

$-1.90409400$

3. 21254900

3. 98205600

$-0.45462700$

3.86014100

2.34393800

$-0.92363900$

4.43547900

5.45770600

$-1.97432600$

$-2.22446500$

5.93725600

$-1.95349600$

$-0.52158800$

5.16662800

$-3.41294500$

$-1.18634000$

1.30351600

1.08014600

$-2.08353600$

0.47169000

0.26046000

$-2.59094500$

0.12654200

$-0.84578900$

$-2.07846000$ 


\begin{tabular}{|c|c|c|c|}
\hline $\mathrm{C}$ & -0.20008100 & 0.69520300 & -3.87677000 \\
\hline $\mathrm{H}$ & -1.23886700 & 0.96191400 & -3.64153500 \\
\hline $\mathrm{H}$ & 0.30281600 & 1.55791800 & -4.31616900 \\
\hline $\mathrm{H}$ & -0.21734600 & -0.13966300 & -4.58307000 \\
\hline $\mathrm{Ag}$ & 0.94522100 & -1.94018900 & 0.01145600 \\
\hline $\mathrm{C}$ & -3.53819700 & -1.34574700 & 0.72729400 \\
\hline $\mathrm{C}$ & -5.84386300 & -1.01662900 & 2.29246200 \\
\hline $\mathrm{C}$ & -4.54204400 & -0.43732600 & 0.32111400 \\
\hline $\mathrm{C}$ & -3.69606300 & -2.04641700 & 1.93392800 \\
\hline $\mathrm{C}$ & -4.84228500 & -1.89789100 & 2.70712800 \\
\hline $\mathrm{C}$ & -5.68460100 & -0.28835700 & 1.11525000 \\
\hline $\mathrm{H}$ & -2.89944500 & -2.71333300 & 2.24513200 \\
\hline $\mathrm{H}$ & -4.95137900 & -2.46020000 & 3.62952500 \\
\hline $\mathrm{H}$ & -6.44098500 & 0.42176200 & 0.79486200 \\
\hline $\mathrm{H}$ & -6.74017200 & -0.88382500 & 2.89175400 \\
\hline $\mathrm{C}$ & -2.26426000 & -1.57399700 & -0.01652800 \\
\hline 0 & -1.26965000 & -2.00321500 & 0.58116500 \\
\hline 0 & -2.29544600 & -1.29328700 & -1.30204000 \\
\hline $\mathrm{H}$ & -1.36364100 & -1.23524800 & -1.70688100 \\
\hline $\mathrm{C}$ & -1.15472200 & 1.76111800 & -0.39473300 \\
\hline $\mathrm{H}$ & -1.09336600 & 2.03423500 & -1.45248600 \\
\hline $\mathrm{H}$ & -0.79746300 & 0.73304800 & -0.31949600 \\
\hline $\mathrm{C}$ & -2.64408100 & 1.81140000 & -0.00795400 \\
\hline $\mathrm{H}$ & -2.97825400 & 2.84946000 & 0.10211000 \\
\hline $\mathrm{H}$ & -2.82590500 & 1.31439900 & 0.94783100 \\
\hline $\mathrm{C}$ & -3.48105900 & 1.24799700 & -1.15918700 \\
\hline 0 & -3.30774300 & 1.66115600 & -2.30209900 \\
\hline $\mathrm{N}$ & -4.47213500 & 0.32925900 & -0.88290300 \\
\hline $\mathrm{C}$ & -5.21930900 & -0.20297300 & -2.02932200 \\
\hline 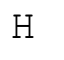 & -5.41983100 & 0.60730100 & -2.73037500 \\
\hline & -4.63162800 & -0.97592400 & -2.53825900 \\
\hline $\mathrm{H}$ & -6.15833100 & -0.63217200 & -1.67285700 \\
\hline & 2.93381300 & 2.74092200 & 1.71479600 \\
\hline
\end{tabular}

\section{$o_{1}$-TS4-cf6}

$\mathrm{Pd}$
$\mathrm{C}$
$\mathrm{C}$
$\mathrm{C}$
$\mathrm{C}$
$\mathrm{C}$
$\mathrm{C}$
$\mathrm{H}$

2.39265000

0.35670500

$-0.11770800$

2.14784700

3. 31627300

0.04169000

1.58038400

2. 19718100

0.69626800

0.22479100

2.27317600

1.11161300

$-0.51539700$

3. 42988500

0.83659300

0.06088100

4.50616700

0.16144200

1. 40389000

4.45921900

$-0.22838200$

$-1.55384900$

3.49357600

1.15393500 
$-0.53470500$

1.85741500

2.97833100

2.79333600

2.84453800

3.33896300

3. 39236400

3. 05861200

1.93699400

4.18128900

3.82643200

3.97784000

2. 41387000

4.83483200

4.78211000

3.77092600

2.00837400

0.86869500

$-0.06347700$

0.62664800

$-0.05619400$

0.16272500

1.56792900

0.14616000

$-4.22176400$

$-6.52603300$

$-4.32476200$

$-5.26241400$

$-6.41727600$

$-5.47867800$

$-5.15470300$

$-7.22507600$

$-5.53589800$

$-7.41853800$

$-2.98239200$

$-2.33712800$

$-2.62378300$

$-1.64083100$

$-0.40324800$

0.26810500

$-0.52491300$

$-1.76567100$

$-1.77475900$

$-2.60017000$
5.39192700

5.30509000

0.06069800

1.31241000

$-0.58614400$

$-0.72893900$

$-1.21262600$

$-2.44608900$

$-2.93202400$

$-3.40490100$

$-1.66569300$

$-0.13153100$

$-0.95890300$

$-2.95664700$

$-3.59794800$

$-4.35108100$

1.20603000

1.08823600

0.33510800

1.97479200

1.49047500

2.90444600

2.22592000

$-1.60232800$

$-0.15324900$

0.47367600

$-0.53745300$

0.57616600

0.87335600

$-0.21718300$

0.89970300

1.42283000

$-0.51013800$

0.71223700

$-0.46056600$

$-1.49462000$

0.52478000

0.40569200

1.14383100

0.28454500

1. 45433400

0.69514600

0.90383600

1.26424400
$-0.04630100$

$-0.73798300$

2.73183000

2.85441800

1.64965500

3.96909300

$-1.05839900$

$-1.05885000$

$-0.77548100$

$-1.43139600$

3.69491600

4. 62325500

4.51035200

$-2.18367400$

$-0.53484700$

$-1.78984500$

$-1.94869500$

$-2.49731600$

$-2.09526200$

$-3.70448400$

$-4.40689100$

$-3.35264500$

$-4.19682700$

$-0.56825200$

$-0.58870200$

0.87660700

0.76491600

$-1.17777000$

$-0.45780500$

1.48518700

$-2.20779900$

$-0.93200500$

2.52933000

1.44797600

$-1.36804800$

$-1.21152700$

$-2.17916100$

$-2.40450800$

1.90072200

1. 91133200

2. 94719000

1.33506600

0.25986500

1. 75686200 
$-2.00123200$

$-1.06223300$

$-3.28655000$

$-3.47357100$

$-3.20174000$

$-2.83491900$

$-4.52200300$

3.18865500

2. 30267300
$-0.80432200$

$-1.58152400$

$-1.27306800$

$-2.73025000$

$-3.17560400$

$-3.14246200$

$-2.93860500$

3. 27271200

1.72928900
1.51936800

1.73191900

1.42613300

1.48335400

0.52022700

2. 26432700

1. 70718700

$-0.26773700$

1. 72812600

\section{$o_{1}$-TS4-cf7}

$\mathrm{Pd}$

C

C

C

C

C

C

$\mathrm{H}$

$\mathrm{H}$

$\mathrm{H}$

$\mathrm{H}$

C

O

O

C

O

C

O

C

$\mathrm{H}$

$\mathrm{H}$

$\mathrm{H}$

$\mathrm{H}$

$\mathrm{H}$

$\mathrm{H}$

O

C

O

C

$\mathrm{H}$

$\mathrm{H}$

$\mathrm{H}$
$-2.42517700$

$-3.23660200$

$-2.01342400$

$-0.94314100$

$-1.13047500$

$-2.35301000$

$-3.41418700$

$-1.57838100$

$-0.30708200$

$-2.47588900$

$-4.36419900$

$-2.44581700$

$-1.41164000$

$-3.23136500$

$-2.72864500$

$-3.07783800$

$-2.82813800$

$-1.90967800$

$-3.76352000$

$-2.46875800$

$-3.79681800$

$-2.13849500$

$-4.17817000$

$-4.59340900$

$-3.24240800$

$-1.65098500$

$-0.60359200$

0.08064500

$-0.13692100$

0.83186600

$-0.85102100$

0.01616000
0.01601600

2.71059800

2.05554300

2.81578700

4.18373000

4.80222000

4.06657800

1.31785900

4.76914700

5.86234500

4.54841000

$-0.00124900$

0.74493700

$-0.27062300$

$-0.63825000$

$-1.93361600$

$-2.88787300$

$-2.92460800$

$-4.08251300$

$-1.69982300$

$-0.57556200$

$-0.17467700$

$-4.17800200$

$-3.91802000$

$-4.99641500$

0.25275600

$-0.36774400$

$-1.15116800$

$-0.08791700$

0.42245500

0.53431000

$-1.03474100$
0.42960400

$-0.34465800$

$-0.06170300$

0.47598700

0.71072100

0.44798200

$-0.08840500$

$-1.15798400$

1.11494500

0.65565700

$-0.30330600$

$-2.42166000$

$-2.29839400$

$-1.46534600$

$-3.75667800$

0.86839800

0.06109600

$-0.80632700$

0.14574800

$-3.67007100$

$-3.97953000$

$-4.54800300$

1.15148800

$-0.55206200$

$-0.14792300$

2. 31251400

2.69023000

1.97467200

4.10498300

4.05153100

4.64580600

4. 63178300 


$\begin{array}{lrrr}\text { Ag } & -0.14775500 & -1.61577400 & -0.47394600 \\ \mathrm{C} & 4.31134300 & -0.69806800 & 0.11728700 \\ \mathrm{C} & 6.95905600 & -0.13692300 & -0.57053800 \\ \mathrm{C} & 4.60971800 & 0.44466600 & -0.65353900 \\ \mathrm{C} & 5.34388000 & -1.52073300 & 0.57216000 \\ \mathrm{C} & 6.66632000 & -1.25172600 & 0.21552100 \\ \mathrm{C} & 5.93456200 & 0.71738100 & -0.98649300 \\ \mathrm{H} & 5.10130400 & -2.37763200 & 1.19277200 \\ \mathrm{H} & 7.46282700 & -1.90594800 & 0.55735900 \\ \mathrm{H} & 6.15662700 & 1.61491600 & -1.55616800 \\ \mathrm{H} & 7.98722500 & 0.08428200 & -0.84272700 \\ \mathrm{C} & 2.89150600 & -1.02848900 & 0.42754600 \\ \mathrm{O} & 2.02842900 & -0.95001600 & -0.46051900 \\ \mathrm{O} & 2.66447700 & -1.40279800 & 1.66428400 \\ \mathrm{H} & 1.67001800 & -1.40534900 & 1.86592500 \\ \mathrm{C} & 0.41519100 & 2.21396200 & 0.72607600 \\ \mathrm{H} & 0.87778700 & 2.66770100 & 1.60671700 \\ \mathrm{H} & 0.33153200 & 1.14676400 & 0.92840200 \\ \mathrm{C} & 1.37281000 & 2.38409100 & -0.47104900 \\ \mathrm{H} & 1.46562800 & 3.44358300 & -0.74661100 \\ \mathrm{H} & 0.96641700 & 1.86131000 & -1.34185900 \\ \mathrm{C} & 2.74332800 & 1.83482800 & -0.07895200 \\ \mathrm{O} & 3.08982800 & 1.79715900 & 1.09712500 \\ \mathrm{~N} & 3.56687800 & 1.33459600 & -1.07453600 \\ \mathrm{C} & 3.15831100 & 1.25639300 & -2.47845800 \\ \mathrm{H} & 2.45060400 & 0.43370900 & -2.64008000 \\ \mathrm{H} & 2.70808700 & 2.19865600 & -2.79819400 \\ \mathrm{H} & 4.04691500 & 1.08333500 & -3.08970300 \\ \mathrm{H} & -4.05017000 & 2.13793400 & -0.78318600\end{array}$

\section{$o_{1}$-TS4-cf8}

$\mathrm{Pd}$
$\mathrm{C}$
$\mathrm{C}$
$\mathrm{C}$
$\mathrm{C}$
$\mathrm{C}$
$\mathrm{C}$
$\mathrm{H}$
$\mathrm{H}$
$\mathrm{H}$
$\mathrm{C}$
$\mathrm{O}$

$$
\begin{array}{r}
-2.13870400 \\
-1.01661800 \\
-0.82150200 \\
0.45109800 \\
1.46421200 \\
1.26686700 \\
0.01599900 \\
2.42390400 \\
2.08699500 \\
-0.15768200 \\
-3.87492200 \\
-2.93432300
\end{array}
$$$$
0.34487200
$$$$
0.59427200
$$$$
\text { 1. } 55271500
$$$$
-1.73219100
$$$$
1.77414900
$$$$
-0.33725400
$$$$
2.20551300
$$$$
0.10934900
$$$$
\text { 2. } 40564100
$$$$
-0.84843200
$$$$
2.12203300
$$$$
-2.19669700
$$$$
1.69970100
$$$$
-2.65353200
$$$$
2.79072900
$$$$
-0.52815300
$$$$
2.25873700
$$$$
-2.89622100
$$$$
\text { 1. } 51353100
$$$$
-3.71000100
$$$$
2.49183700
$$$$
-0.19135400
$$

3.25455200

0.20181100 
$-3.76650200$

$-5.21399200$

$-3.34166300$

$-3.51010900$

$-2.93217200$

$-4.50063400$

$-5.74468100$

$-5.07205700$

$-5.80989400$

$-3.93709900$

$-5.18641200$

$-5.05254300$

$-0.77631600$

$-0.32893400$

$-0.25804800$

0.14977000

$-0.61566100$

1.07407900

0.28743900

$-0.78704900$

3.79949600

6.35373400

4.27849100

4.59124200

5.87126600

5.55633200

4.19648100

6.48586600

5.89996900

7.34539300

2.43951600

1.60133300

2.26118700

1.28958300

0.70780700

1.16442700

$-0.25029400$

1. 62307900

1.11329200

1.77105000

2.97637000

3.58802400

3.38872500

3.44338800
1.23199700

3.11563600

$-1.32035900$

$-2.12806400$

$-2.09745100$

$-3.25386100$

3.28237200

4.08480300

2.44875800

$-4.15261000$

$-3.00325200$

$-3.47425600$

$-0.44963900$

$-1.64253800$

$-2.36523000$

$-2.21815200$

$-2.91745300$

$-2.78254100$

$-1.43975900$

$-1.33460000$

$-0.77422900$

0.35384200

$-0.07031700$

$-0.87906000$

$-0.32616700$

0.49042400

$-1.39959500$

$-0.41975300$

1.06405300

0.79709600

$-1.39774000$

$-1.14959700$

$-2.26295400$

$-2.49198900$

2.57436100

3.57006800

2.62984200

1.61753500

0.66401600

2.06123800

1.45640800

2.42570600

0.14035600

$-0.82734100$
$-0.32797300$

$-0.50386700$

0.96787200

$-0.01417000$

$-1.13049400$

0.24050400

0.44069400

$-0.98747000$

$-1.12874400$

0.51816100

1.05211400

$-0.67669300$

1. 90707800

1.85791200

0.82840800

3.17882900

3.53456300

3.03136000

3.93164300

$-1.38778600$

$-0.79128200$

$-0.84119400$

0.33271300

$-1.93871700$

$-1.96094800$

0.29662700

$-2.80605900$

$-2.85155700$

1.14933100

$-0.85821400$

$-0.79647400$

$-1.66426700$

0.18831600

0.31279600

1. 56256800

1.58773500

2.08956200

2. 37775200

2. 49858500

3. 37031800

1. 69234200

1. 26509300

1. 44054200

2.54164500 
$\mathrm{H}$

$\mathrm{H}$

$\mathrm{H}$

$\mathrm{H}$

$\mathrm{H}$

\section{$o_{2}$-TS1-a}

C

$\mathrm{H}$

$\mathrm{H}$

C

$\mathrm{H}$

$\mathrm{H}$

C

O

$\mathrm{N}$

C

C

C

C

C

$\mathrm{H}$

C

$\mathrm{H}$

$\mathrm{H}$

$\mathrm{H}$

C

$\mathrm{H}$

$\mathrm{H}$

$\mathrm{H}$

C

O

$\mathrm{O}$

$\mathrm{Pd}$

C

O

O

C

O

O

C

$\mathrm{H}$

$\mathrm{H}$



.

.

(

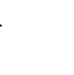

(

.

.

.

(

(n)

.

H

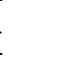

C

P

d

$\mathrm{O}$
$\mathrm{O}$
$\mathrm{C}$
$\mathrm{O}$
$\mathrm{O}$
$\mathrm{C}$
$\mathrm{H}$
$\mathrm{H}$

$\mathrm{H}$
4.38962200

2.62163400

3. 35172100

$-2.01369600$

$-1.87471100$
$-0.73672400$

$-0.64550900$

$-1.83803500$

1.30516600

2. 52046400
3.09362200

3.23649000

2.14691400

$-2.08919500$

0.15253900

$\begin{array}{rrr}1.27182100 & -3.47244900 & 2.19316900 \\ 1.36860600 & -4.55319600 & 2.05436400 \\ 1.46412400 & -3.25518500 & 3.25019200 \\ 2.35857900 & -2.77548400 & 1.35634500 \\ 3.33930100 & -2.91323200 & 1.83345600 \\ 2.17816600 & -1.69817900 & 1.34112300 \\ 2.47616400 & -3.35938400 & -0.05829400 \\ 2.06239600 & -4.48288200 & -0.32276900 \\ 3.15485600 & -2.59713900 & -0.98996400 \\ 3.44766600 & -1.21612600 & -0.76501100 \\ 2.43823500 & -0.24371700 & -0.80421700 \\ 4.75142100 & -0.83851500 & -0.42474100 \\ 2.70442900 & 1.09527800 & -0.42992700 \\ 5.03826100 & 0.48186600 & -0.07837400 \\ 5.52293500 & -1.60269600 & -0.40112000 \\ 4.01790500 & 1.43297000 & -0.05633800 \\ 2.24933700 & 2.25351300 & -0.98044200 \\ 6.05095500 & 0.76147100 & 0.19847800 \\ 4.24127500 & 2.45867400 & 0.22047300 \\ 3.25760400 & -3.08033100 & -2.36983500 \\ 4.22489900 & -2.78224100 & -2.78593300 \\ 3.17577400 & -4.16703500 & -2.35738700 \\ 2.44664200 & -2.66002400 & -2.97295200 \\ 1.00485900 & -0.64669600 & -0.98059700 \\ 0.20199200 & -0.03764200 & -0.13147800 \\ 0.59085100 & -1.47406300 & -1.78504900 \\ 0.88327400 & 1.77493500 & 0.50377300 \\ -1.97162500 & 2.05796200 & 1.19127500 \\ -2.24937400 & 1.81887400 & -0.01893500 \\ -0.79617600 & 2.14624500 & 1.66664800 \\ 2.00364500 & 4.23181600 & -0.27206500 \\ 1.50439200 & 3.72148100 & 0.78508300 \\ 2.30841000 & 3.57549100 & -1.31627400 \\ 2.22486400 & 5.72730300 & -0.27636000 \\ 1.36343300 & 6.19950700 & -0.76274500\end{array}$




\section{$o_{2}$-TS1-b}

C

$\mathrm{H}$

$\mathrm{H}$

C

$\mathrm{H}$

$\mathrm{H}$

C

O

$\mathrm{N}$

C

C

C

C

C

$\mathrm{H}$

C
2.30166700

$-3.10983700$

$-2.73616800$

$-3.68777800$

$-3.78704300$

$-1.92891600$

$-0.13627800$

$-0.75209800$

$-0.84888400$

$-2.05892400$

$-0.20334300$

$-2.16043000$

$-0.36626100$

$-2.77880600$

$-2.51988500$

$-2.71294600$

$-3.81568300$

$-4.97545200$

$-4.15414900$

$-4.71792500$

$-3.73229000$

$-6.41134200$

$-7.08142000$

$-6.61673300$

$-6.59470400$
6.10835000

0.74328500

2. 26177200

2.17894700

2.38994800

3.19561000

3.14466300

1.88507600

1.40199200

2.13188200

$-0.65300800$

$-3.02972800$

$-1.99598800$

$-3.63766600$

$-1.59776000$

$-1.50261400$

$-3.24691300$

$-4.41456700$

$-2.23087600$

$-0.80297800$

$-3.74758500$

$-1.96593900$

0.40566500

$-0.50021600$

1. 55635800

1. 65485100

0.27358300

0.48686700

1.01698500

$-0.72849400$
$-0.77260400$

1.84737700

2.56916300

0.80425200

2. 28887600

3. 36809000

0.51595300

0.22034400

1.26512000

2.86886500

$-0.27501600$

1.07311900

$-1.30583000$

$-1.48852800$

$-0.72748900$

$-0.45402100$

$-1.75175900$

$-0.91295900$

$-2.52958900$

$-2.14033000$
3.75027900

4.71263300

3.70522000

2.63277800

2.55336700

1.66450700

2. 91966600

4.05503000

1.86541800

0.50733700

$-0.25625400$

$-0.06549000$

$-1.59934700$

$-1.37598900$

0.54342600

$-2.13468200$
1.04785200

1.87118000

1.51329000

1.63702700

0.90106400

2.95586400

2.02809000

1. 46210700

2. 82855700

2.21065500

1. 51948100

1. 45997700

2.70336700

0.11478000

2.73716900

$-0.35112700$

3. 33233200

$-0.50658400$

3.18097200

$-0.08327100$

2.04775700

$-0.40872100$

4.19967700

0.68790900

1.94222100

0.02834600

4.09255500

1.15156700

5.06602800

0.92946300

2.96940800

0.82596700 
H

$\mathrm{H}$

$\mathrm{H}$

C

$\mathrm{H}$

H

$\mathrm{H}$

C

o

0

$\mathrm{Pd}$

C

O

O

C

O

O

C

$\mathrm{H}$

$\mathrm{H}$

$\mathrm{H}$

C

$\mathrm{H}$

H

$\mathrm{H}$

Ag

C

C

C

C

$\mathrm{H}$

C

$\mathrm{H}$

C

$\mathrm{H}$

$\mathrm{H}$

$\mathrm{H}$

\section{$o_{2}$-TS1-c}

C

C

C

C
$-2.65269200$

$-1.80216400$

$-3.16555000$

2.06479700

1.43951700

3.11608200

1. 79827900

0.39460500

$-0.15349800$

1. 37545500

$-2.02723400$

$-1.86278300$

$-0.81085400$

$-2.37280300$

$-4.60968700$

$-4.03404500$

$-4.00320900$

$-6.12192500$

$-6.48322100$

$-6.49104700$

$-6.49636900$

$-2.69770000$

$-3.04782700$

$-3.58273100$

$-2.11906200$

1.07899100

3.64628700

3.19658500

3.96088400

3.07214300

2.94426300

3.83123900

4.29215300

3.39010900

2.74690000

4.09887300

3.40197800
1.55104900

4.88332300

2.89597300

3.87933000

4.76815200

4.14441200

3.12610800

0.81973800

$-0.29655000$

0.81139100

$-0.15276200$

$-3.25203500$

$-3.44001800$

$-2.14489300$

0.86567000

0.11772900

1.56219900

0.89666100

1.88074000

0.15729900

0.63139100

$-4.46935700$

$-4.37469900$

$-4.50924000$

$-5.38644700$

$-2.22082400$

$-0.29869200$

$-1.42379200$

$-0.44342700$

$-2.66967900$

$-1.32386400$

$-1.68263700$

0.42169600

$-2.81296100$

$-3.53413000$

$-1.78173700$

$-3.79949000$
$-0.69722600$

1.76253400

1.15747900

$-1.85253100$

$-1.97659200$

$-1.96222300$

$-2.60108600$

$-0.98316600$

$-0.53270400$

$-1.71272100$

0.23088200

0.26406500

$-0.38915400$

0.65511600

$-0.21072400$

0.64882500

$-1.08058300$

$-0.18661100$

$-0.49277400$

$-0.90681300$

0.80365700

0.65066300

1. 68227200

0.00537700

0.52332700

$-0.69751300$

1.18349300

1.89461700

$-0.17598100$

1.27766900

2. 94733500

$-0.81031900$

$-0.73829800$

$-0.09010200$

1. 84925800

$-1.85842700$

$-0.54901900$

1.17996200

4.28737300

$-1.78041100$

2.46475000

4.38226500

$-1.24143900$

3.07264500

3.26124100

$-0.66967300$

2.41282800

2.02705000

$-0.62048300$ 
1.12402900

0.50997000

0.70880600

2.99794100

4.07171700

0.58638800

$-0.49251800$

3.04639200

2.48388500

4.06457300

3.10415700

3. 84291600

2.14189600

3. 57476100

4.69107800

2.69883800

1. 35485900

0.33128300

1.07358800

$-0.95367500$

$-0.18612300$

1.87807900

$-1.18336000$

$-2.17832900$

$-0.38102200$

$-2.16261100$

3. 05034000

2.79152100

4.12393100

2.51388000

0.64368300

$-0.02663000$

1.45198100

$-1.62944600$

$-1.17482400$

$-1.93364700$

$-0.14983100$

0.01296300

$-4.07395500$

$-3.45030900$

$-3.55676200$

$-5.53531400$

$-5.81068200$

$-6.13038600$
1.94682800

3. 06027400

5.15652800

5.32915100

3. 34650800

1.00332200

2.96818300

0.82802600

0.59988500

1.06114100

$-0.43699900$

$-0.28245800$

$-0.61384800$

$-1.63197400$

$-1.63516700$

$-2.69734100$

$-2.67938600$

$-1.97842800$

$-3.33020600$

$-1.86666000$

$-3.23775300$

$-3.87145900$

$-2.48422900$

$-1.92505500$

$-3.73141700$

$-2.40288700$

$-3.77862300$

$-4.74524500$

$-3.72702000$

$-3.64635200$

$-1.12012900$

0.03009300

$-1.39018200$

0.06678400

2.92750000

2.14735100

2.57431300

1.58218700

$-1.15879900$

$-0.09328400$

$-2.12943900$

$-1.24054800$

$-2.27572600$

$-0.89684200$
$-1.17059600$

$-1.74373900$

$-2.23244800$

$-1.26760200$

$-0.24871200$

$-1.14630100$

$-2.15402600$

0.05499500

0.96692200

0.38039500

$-0.83437300$

$-1.62842300$

$-1.31746300$

$-0.00949600$

0.49095300

0.14982500

$-0.33060600$

0.32646700

$-1.54027800$

$-0.26832300$

$-2.12904300$

$-2.02953000$

$-1.51273500$

0.29519300

$-3.07676600$

$-1.97263800$

1.07343600

0.62826200

1.25345700

2. 01810000

1.52442000

1.49248500

2. 38859200

0.27060500

1.23183900

0.62481900

1.96292300

1.93428000

$-0.34313500$

$-0.67690800$

0.28851300

$-0.72186400$

$-0.93384700$

0.13215500 
H

C

$\mathrm{H}$

$\mathrm{H}$

$\mathrm{H}$

\section{$o_{2}$-TS1-d}

C

C

C

C

C

C

$\mathrm{H}$

$\mathrm{H}$

$\mathrm{H}$

$\mathrm{H}$

$\mathrm{H}$

C

$\mathrm{H}$

$\mathrm{H}$

C

$\mathrm{H}$

$\mathrm{H}$

C

O

$\mathrm{N}$

C

C

C

C

C

$\mathrm{H}$

C

$\mathrm{H}$

H

$\mathrm{H}$

C

$\mathrm{H}$

$\mathrm{H}$

$\mathrm{H}$

C

O
$-5.74791900$

$-1.37705100$

$-1.20505200$

$-0.63066600$

$-2.37736200$
$-0.59443500$

4.41169400

4.88234000

4.79858000

4.63366800
$-1.57526600$

1.13805600

2.10920900

0.43402500

0.76530200

$-0.47693800$

4.56317700

1.44397200

$-1.81962100$

4.84911000

1.70917400

$-2.82936700$

4.14575200

1.05067400

$-2.51310000$

3.15024100

0.11574400

$-1.16336100$

2. 88320600

$-0.15010800$

$-0.14673400$

3.57873200

0.50999100

0.30870600

5.10844400

1.96060700

$-2.08113600$

5.62135600

2.42866500

$-3.87347600$

4.37021400

1.26214700

$-0.89236800$

2.13233100

$-0.88544200$

0.89092200

3. 34071900

0.29262300

$-3.60667500$

2. 33138100

$-0.53538600$

$-3.20801200$

1.82953000

$-1.42477100$

$-4.43789400$

2.95800900

$-0.87535600$

$-4.16738700$

1.27478200

0.44637700

$-4.71034600$

1.79196200

1.24658200

$-3.34291800$

0.74119400

0.92270200

$-5.17019700$

0.34846200

$-0.23244300$

$-6.12916700$

0.79898600

$-0.84415100$

$-4.96813600$

$-1.02308100$

$-0.11041400$

$-3.78251500$

$-1.58550500$

0.44826300

$-2.54494400$

$-1.56241700$

$-0.23158900$

$-3.83956200$

$-2.09681200$

1.74752700

$-1.34004200$

$-1.96732100$

0.41563200

$-2.67832900$

$-2.51844200$

2. 38831200

$-4.79775000$

$-2.10366400$

2.25770000

$-1.44746600$

$-2.43292000$

1. 73646000

$-0.19166000$

$-2.61254000$

0.04883100

$-2.72667700$

$-2.88895300$

3.40843800

$-0.54930900$

$-2.76866100$

2.24175500

$-5.90546000$

$-1.94721900$

$-0.74898200$

$-6.14287300$

$-2.76797600$

$-0.06428400$

$-6.81050700$

$-1.39086100$

$-0.99250600$

$-5.47577300$

$-2.35902400$

$-1.66930500$

$-2.40337900$

$-0.85707600$

$-1.51787800$

$-1.38404000$

$-0.20013700$

$-1.78687900$ 


\begin{tabular}{|c|c|c|c|}
\hline 0 & -3.40733200 & -0.91393900 & -2.38415600 \\
\hline $\mathrm{H}$ & -3.18021000 & -0.34254600 & -3.14439300 \\
\hline $\mathrm{C}$ & 3.00395000 & -0.43845300 & 0.40394000 \\
\hline $\mathrm{N}$ & 1.86013000 & -1.36021900 & 0.43182500 \\
\hline $\mathrm{C}$ & 1.89405500 & -2.66584500 & 0.64108800 \\
\hline 0 & 0.89273700 & -3.41528900 & 0.37431600 \\
\hline $\mathrm{C}$ & 3.09599100 & -3.34558700 & 1.26081000 \\
\hline $\mathrm{H}$ & 3.56693500 & -3.99788200 & 0.51823100 \\
\hline $\mathrm{H}$ & 3.83824500 & -2.64475000 & 1.64643700 \\
\hline $\mathrm{H}$ & 2.73714900 & -3.98275600 & 2.07414300 \\
\hline C & 2.46409100 & 0.95142600 & -0.00113500 \\
\hline O & 3.02645100 & 1.98135000 & 0.32040300 \\
\hline 0 & 1.41663500 & 0.91480400 & -0.81037400 \\
\hline $\mathrm{H}$ & 3.47606100 & -0.36692200 & 1.38906500 \\
\hline $\mathrm{Pd}$ & 0.22298700 & -0.65419000 & -0.437149 \\
\hline $\mathrm{C}$ & 4.05881100 & -0.85280600 & -0.6705360 \\
\hline $\mathrm{H}$ & 4.21399900 & -1.93403800 & -0.61165700 \\
\hline $\mathrm{H}$ & 3.62061000 & -0.65288200 & -1.65534400 \\
\hline C & 5.38726500 & -0.15079300 & -0.50565900 \\
\hline C & 6.40088900 & -0.74528400 & 0.25810500 \\
\hline C & 5.62828600 & 1.10237600 & -1.08318300 \\
\hline $\mathrm{C}$ & 7.62803500 & -0.10700700 & 0.44307400 \\
\hline $\mathrm{H}$ & 6.22955700 & -1.72278400 & 0.70620400 \\
\hline C & 6.85421600 & 1.74289100 & -0.90209500 \\
\hline $\mathrm{H}$ & 4.84624000 & 1.58467000 & -1.66133300 \\
\hline & 7.85761000 & 1.14120800 & -0.13917700 \\
\hline & 8.40369400 & -0.58528600 & 1.0356020 \\
\hline & 7.02405600 & 2.71581600 & -1.35526700 \\
\hline & 8.81239200 & 1.64138400 & -0.000170 \\
\hline
\end{tabular}

\section{$o_{2}$-TS1-e}

$\mathrm{C}$
$\mathrm{C}$
$\mathrm{C}$
$\mathrm{C}$
$\mathrm{C}$
$\mathrm{C}$
$\mathrm{H}$
$\mathrm{H}$
$\mathrm{H}$
$\mathrm{H}$
$\mathrm{H}$
$\mathrm{C}$

-0.87156700
-2.19553700
-3.19032500
-2.88059500
-1.55116500
-0.54772800
-0.09795100
-2.45467900
-4.21890600
-1.28678000
0.47459700
-3.94438300

4.72058000

1.00810800

4.86804500

1.43269300

4.02319700

0.93783500

3.01971200

0.00907800

2.89266000

$-0.41541900$

3. 73012700

0.07843900

5.37802500

1.39614300

5.64386200

2.14905200

4.14219400

1.27356700

2.13792900

$-1.14806000$

3. 59957200

$-0.26640300$

2.05293800

$-0.46585400$ 
$-3.60342100$

$-4.87582300$

$-4.25546500$

$-4.74764000$

$-3.32098600$

$-5.22290500$

$-6.29242600$

$-4.84614600$

$-3.57157800$

$-2.42356100$

$-3.44158200$

$-1.12581900$

$-2.17972200$

$-4.33670900$

$-1.04026800$

0.02449100

$-2.08100600$

$-0.06015500$

$-5.72633300$

$-5.88407800$

$-6.67738500$

$-5.28418900$

$-2.60052500$

$-3.37369200$

$-1.70234700$

$-1.73833600$

2. 95929200

1.94842700

2.14948700

1.22248500

3.47892500

3.95379400

4.16796800

3.27704500

2.27503900

2. 81420400

1.13850300

3. 34183800

0.16217100

4.15967100

4.07730500

4.06301400

5.51687700

6.47436900
1.54481100

2.56731200

0.99658100

1.49220800

0.58180000

$-0.06704100$

0.24772200

$-1.40069700$

$-1.78329600$

$-1.74152800$

$-2.09604900$

$-1.90291400$

$-2.29555500$

$-2.12426000$

$-2.17507600$

$-2.47063600$

$-2.51344500$

$-2.32868200$

$-2.46303300$

$-3.21193600$

$-2.00521900$

$-2.94174000$

$-1.32632000$

$-1.72007900$

$-0.29573800$

$-0.07733600$

0.08659100

$-0.97215000$

$-2.24312300$

$-3.11631500$

$-2.72920700$

$-3.37207500$

$-1.92557000$

$-3.34653800$

1. 33631800

2.42846000

1. 12260300

0.35653100

$-0.49345000$

$-0.28433200$

$-1.33585600$

0.29026900

$-0.01671000$

$-1.03359400$
$-1.37521100$

$-0.72379700$

0.62036000

1.46589200

1.00143600

0.10863500

$-0.39388100$

0.24521800

0.75690700

$-0.05487100$

2.11330200

0.49400400

2.66766200

2.72706100

1.87167100

0.05991500

3.72728600

2. 30931600

$-0.24482600$

0.53937200

$-0.51668300$

$-1.12411000$

$-1.47213200$

$-2.30095100$

$-1.76346800$

$-2.71627300$

$-0.04348600$

0.09205800

0.40164000

0.30014800

0.93691700

0.18851700

1.19858500

1.81665000

$-0.64310100$

$-0.59311200$

$-1.27846700$

0.94655800

$-0.60831500$

$-0.98584300$

$-1.27471200$

$-1.91418600$

$-0.36918600$

$-0.27063000$ 
5.83570700

7.72604000

6.24114800

7.08380500

5.09614400

8.03336100

8.45723300

7.31731800

9.00540900
1.26271300

$-0.78526200$

$-2.02827900$

1. 50964100

2.05553600

0.48799600

$-1.58667300$

2.50500700

0.68423100
0.11297200

0.29863500

$-0.64491800$

0.68470600

0.03345700

0.77964700

0.36598400

1.05361000

1.22419200

\section{$o_{2}$-TS1-f}

C

C

C

C

C

C

$\mathrm{H}$

$\mathrm{H}$

$\mathrm{H}$

$\mathrm{H}$

$\mathrm{H}$

C

$\mathrm{H}$

$\mathrm{H}$

C

$\mathrm{H}$

$\mathrm{H}$

C

O

$\mathrm{N}$

C

C

C

C

C

$\mathrm{H}$

C

$\mathrm{H}$

$\mathrm{H}$

$\mathrm{H}$

C

$\mathrm{H}$
0.95082500

0.15259300

$-1.17484900$

$-1.73241200$

$-0.91495500$

0.40482300

1. 97865700

0.57047100

$-1.77716800$

$-1.32001700$

1.01346200

$-3.16465600$

$-3.18687700$

$-3.78742800$

$-3.82216500$

$-4.18335400$

$-3.09118100$

$-5.03740500$

$-5.96625500$

$-5.03613200$

$-3.92883000$

$-2.74157600$

$-4.00731400$

$-1.59418300$

$-2.89354700$

$-4.94122900$

$-1.68561900$

$-0.64275100$

$-2.96218500$

$-0.81200400$

$-6.06397700$

$-6.42345700$
3.71014600

3.15613700

2.80916700

2.99026900

3.52904000

3.89810100

3. 99274200

2. 98958300

2.38684900

3.64733800

4.32103400

2. 62800900

2.25309800

3.53117200

1.58992000

2.07836500

0.83881900

0.97932300

1.69101700

$-0.39069400$

$-1.22242600$

$-1.23132300$

$-1.96517600$

$-1.90937500$

$-2.64507900$

$-1.95197200$

$-2.57688100$

$-2.65302700$

$-3.19951800$

$-3.09159300$

$-0.98162200$

$-1.91814100$
0.83535600

1. 84273100

1.57777300

0.30368400

$-0.70274000$

$-0.44324300$

1. 04697600

2.83123600

2.37645700

$-1.70382000$

$-1.23853000$

$-0.03377900$

$-1.06129300$

$-0.03645900$

0.90318200

1. 81542400

1.20541400

0.20789800

$-0.15015600$

$-0.00661200$

0.34226200

$-0.40644800$

1.52678200

0.08982500

2.01970600

2.08144600

1.33193100

$-0.49773600$

2. 95136300

1.71638900

$-0.86560900$

$-0.42576700$ 
$-6.88479500$

$-5.64339600$

$-2.56509400$

$-3.42750100$

$-1.41273400$

2.81344500

1.54824900

1.44524200

0.37311400

2. 56920200

2.99951300

3. 36403000

2.13908500

2. 49460500

2.86762300

1.71951200

3.32195100

0.00424800

3.76960100

4.05698400

3.19135900

4.99624500

6.09364600

5.03684500

7.20721600

6.07601000

6.14981300

4.18909800

7.23735700

8.05185300

6.16819800

8.10493100

1.45069800
$-0.26900900$

$-1.17087100$

$-0.26142700$

$-0.02887100$

0.37151800

$-1.09975200$

$-1.78637100$

$-3.08814000$

$-3.60472600$

$-4.06374000$

$-4.40707100$

$-3.64548400$

$-4.93629500$

0.36073900

0.99319800

0.90852400

$-1.49425900$

$-0.61484200$

$-1.13738600$

$-2.17998400$

$-0.81653700$

$-0.27401300$

$-0.74161200$

1.02654000

0.07105500

$-1.75178600$

1.84214900

1.39905400

1.36660500

$-0.30683900$

2. 84708700

1.99998500

1.82868700
$-0.94418900$

$-1.85829200$

$-1.55196300$

$-2.38340800$

$-1.48881200$

0.50010900

0.23513900

$-0.00273400$

$-0.46330900$

0.28545500

$-0.66116600$

0.90655100

0.78365200

0.81917200

1.76748900

$-0.18905100$

1.38610600

$-0.44121400$

$-0.73010000$

$-0.89774600$

$-1.60276700$

$-0.54850600$

0.18653100

$-1.06817400$

0.39911100

0.59107500

$-0.85801400$

$-1.63830300$

$-0.12298400$

0.96880900

$-1.27097800$

0.03996800

0.06703600

\section{$o_{2}$-TS1-g}

C

C

C

C

C

C

$\mathrm{H}$

$\mathrm{H}$
1.31374700

0.42333700

$-0.82999400$

$-1.22624000$

$-0.30958000$

0.93905600

2. 26589600

0.69906500
3.97615300

3.40226400

2. 94837200

3.06190700

3.61433400

4.07116100

4.38160600

3.31914600
1.17436200

2.08909200

1. 66568200

0.32555900

$-0.58612500$

$-0.17356500$

1. 51229400

3.13744900 
$-1.50537300$

$-0.58901300$

1. 62204200

$-2.58969600$

$-2.43843600$

$-3.17473300$

$-3.43180400$

$-3.74131300$

$-2.83883000$

$-4.72415900$

$-5.51138000$

$-4.96917000$

$-3.99289500$

$-2.86170300$

$-4.10519500$

$-1.75897500$

$-3.05416200$

$-4.99867600$

$-1.87131000$

$-0.77352000$

$-3.14013700$

$-1.03787200$

$-6.13453800$

$-6.63139500$

$-6.81519600$

$-5.81387100$

$-2.68050300$

$-3.56289700$

$-1.48398100$

2. 62273200

1.37985300

1.27060800

0.20100100

2.36408300

2. 85500100

3.11761800

1.89870600

2.22895900

1.46753100

2.76711600

3.06202500

$-0.12353000$

3.67850100

3.98905500
2.51591000

3.68397800

4.50520400

2.63692000

2.08334300

3.52594900

1. 78678700

2.39553700

0.95594000

1.33095700

2.16148300

$-0.03041500$

$-0.99262700$

$-1.25589700$

$-1.56418100$

$-1.95994100$

$-2.30748900$

$-1.35984300$

$-2.44833800$

$-2.82444900$

$-2.73417000$

$-2.98689600$

$-0.51347300$

$-1.30724600$

0.32654000

$-0.89421800$

$-0.53444700$

$-0.41731800$

0.00388200

$-1.10567500$

$-1.85638900$

$-3.17503900$

$-3.75029400$

$-4.09188000$

$-4.57691100$

$-3.57455300$

$-4.87753600$

0.35888000

0.71980400

1.20031600

$-1.25257600$

$-0.82979400$

$-1.41581500$

$-2.45830800$
2.39718800

$-1.63430900$

$-0.89909000$

$-0.18611700$

$-1.11771100$

$-0.45275700$

0.78060300

1.63904100

1.16680100

0.09610800

$-0.33917700$

0.01479500

0.41832600

$-0.37060000$

1.68988200

0.18356000

2. 22555700

2. 27305000

1.50378800

$-0.27719900$

3.22109800

1.94079300

$-0.72805700$

$-0.15922300$

$-0.86605400$

$-1.70177000$

$-1.68628300$

$-2.51894200$

$-1.79651400$

0.53055300

0.34628400

0.34822700

$-0.05419500$

0.85433500

0.00389600

1.45278200

1.45475700

0.36756300

$-0.53803400$

1. 22191100

1.52383900

$-0.57805100$

$-0.57611500$

$-0.46294100$ 
$\mathrm{H}$

C

C

C

C

$\mathrm{H}$

C

$\mathrm{H}$

C

$\mathrm{H}$

$\mathrm{H}$

$\mathrm{H}$

$\mathrm{H}$

\section{$o_{2}$-TS1-h}

C

C

C

C

C

C

$\mathrm{H}$

$\mathrm{H}$

$\mathrm{H}$

$\mathrm{H}$

$\mathrm{H}$

C

$\mathrm{H}$

$\mathrm{H}$

C

$\mathrm{H}$

$\mathrm{H}$

C

O

$\mathrm{N}$

C

C

C

C

C

$\mathrm{H}$

C

$\mathrm{H}$
3.16396400

4.87743900

5.92022000

4.93823900

7.00100800

5.88617300

6.01851200

4.13250500

7.05183400

7.80438400

6.05435700

7.89456300

2.37636100
$-1.32976400$

$-0.49796200$

$-0.74568600$

0.64714700

0.13094200

$-1.63432300$

1. 52604400

0.85019800

1.27054000

$-0.07671900$

2. 40710700

1. 95245500

2.09601000
$-1.53933100$

$-0.51725200$

0.38507200

$-1.32261000$

0.48258700

1.01252900

$-1.22770100$

$-2.02435700$

$-0.32403500$

1.18419800

$-1.86268700$

$-0.25136900$

1.05713300

$-0.37253800$

$-3.30564700$

3.55434900

0.87951600

$-3.44667100$

4.15532100

2.01635300

$-2.90856100$

3.54482000

1.91623800

$-2.22656300$

2.32756900

0.64986000

$-2.09085300$

1.73471800

$-0.49039500$

$-2.62304600$

2.33887200

$-1.25691300$

$-3.72106900$

4.03035300

0.97549900

$-3.97567400$

5.10045600

2.99049000

$-3.02387900$

4.01608200

0.54095100

$-1.56143500$

0.79308500

$-1.45959000$

$-2.48875200$

1.86657700

3.13678700

$-1.64443600$

1.64498500

3.16153000

$-2.00521700$

0.61005500

4.05955700

$-2.00500800$

2.11042000

3.12082100

$-0.10631000$

1.64063300

3. 31976500

0.28366100

2.64769400

2.11519000

0.24134200

1.39478200

4.14067900

0.50778000

0.68262100

5.00381700

$-0.19139800$

0.11576700

4.08830900

1.85818100

0.47371000

2.86100600

2. 57830900

0.69004200

1.73111900

2. 31191900

$-0.10893800$

2.77193500

3. 46202800

1.76564500

0.46081200

2. 82817600

0.24858300

1.54335700

4.03621900

2.08639900

3.65710400

3.65142100

2.36572000

0.40411100

3.69570000

1. 35681400

$-0.70830600$

3.19788100

$-0.39026800$ 
H

$\mathrm{H}$

C

$\mathrm{H}$

$\mathrm{H}$

$\mathrm{H}$

C

O

O

C

$\mathrm{N}$

C

O

C

$\mathrm{H}$

$\mathrm{H}$

$\mathrm{H}$

C

O

O

$\mathrm{H}$

$\mathrm{Pd}$

C

H

$\mathrm{H}$

C

C

C

C

$\mathrm{H}$

C

$\mathrm{H}$

C

$\mathrm{H}$

$\mathrm{H}$

$\mathrm{H}$

Ag

C

o

O

$\mathrm{H}$

C

$\mathrm{H}$

$\mathrm{H}$
1.46608600

$-0.55455000$

5.08700100

5.15274600

6.05184900

4.78803900

1.82920300

0.89821500

2.78828400

$-3.69987500$

$-2.64911000$

$-2.82827400$

$-1.86603700$

$-4.16311100$

$-4.56553800$

$-4.89571100$

$-3.98998000$

$-3.00150500$

$-3.53075800$

$-1.85182800$

$-4.35699200$

$-0.85613900$

$-4.53307600$

$-4.92069400$

$-3.83598200$

$-5.68001300$

$-6.94175700$

$-5.50270600$

$-8.00394300$

$-7.09429500$

$-6.56157800$

$-4.52453700$

$-7.81561900$

$-8.97705300$

$-6.40609100$

$-8.64085800$

3.68009900

1.21174700

2.43741300

0.30498200

0.66872100

0.61957300

0.30964500

$-0.27329300$
4.71485800

4.12262800

2.47693900

3.53908400

1.99582400

2. 36032700

1.21142200

0.31703200

1.12736000

0.85394600

1.87231000

3.15138000

3.89653000

3.83827400

4.12693400

3.22077100

4.75748800

$-0.50292900$

$-1.38843200$

$-0.64612500$

1.07139500

1.10338400

0.72725600

1.71695200

0.43618400

$-0.25109200$

0.17930400

$-1.60450400$

$-0.71863500$

1.22958400

$-2.50510700$

$-1.95307100$

$-2.06528900$

$-0.36677300$

$-3.55226800$

$-2.76725100$

$-1.02720400$

$-3.11245800$

$-2.93445900$

$-2.17337900$

$-1.24537100$

$-4.46419000$

$-4.46933600$

$-4.62577100$
2.93124900

1.63129900

$-0.40463900$

$-0.15658600$

$-0.24158400$

$-1.45091400$

$-1.13025500$

$-1.03348500$

$-1.92311800$

0.37975800

0.23550600

$-0.03727400$

$-0.43972700$

0.15272900

$-0.82372700$

0.67533600

0.71958500

0.62334600

1.27205100

$-0.01133700$

1.22793100

$-0.16779300$

$-0.93142600$

$-1.19398300$

$-1.72591900$

$-0.83283000$

$-0.40298700$

$-1.14708000$

$-0.28804400$

$-0.16007200$

$-1.03564600$

$-1.46756900$

$-0.60566800$

0.04488800

$-1.28127600$

$-0.51933500$

$-1.81319100$

$-1.61922100$

$-1.67145700$

$-1.76182200$

$-1.69446600$

$-1.33731200$

$-0.28477500$

$-1.94635700$ 


\section{$o_{2}$-TS1-i}

C

C

C

C

C

C

$\mathrm{H}$

$\mathrm{H}$

$\mathrm{H}$

$\mathrm{H}$

$\mathrm{H}$

C

$\mathrm{H}$

$\mathrm{H}$

C

$\mathrm{H}$

$\mathrm{H}$

C

O

$\mathrm{N}$

C

C

C

C

C

$\mathrm{H}$

C

$\mathrm{H}$

$\mathrm{H}$

$\mathrm{H}$

C

$\mathrm{H}$

$\mathrm{H}$

$\mathrm{H}$

C

O

O

C

$\mathrm{N}$

C
0.16433600

$-0.88326000$

$-2.08889500$

$-2.27720500$

$-1.21081800$

$-0.00039700$

1.10419300

$-0.76669100$

$-2.89810500$

$-1.31985400$

0.81808800

$-3.56529000$

$-3.39105500$

$-4.33111100$

$-4.16298400$

$-4.61740700$

$-3.39133400$

$-5.26684300$

$-6.22704500$

$-5.11796200$

$-4.01400400$

$-2.76542600$

$-4.18997100$

$-1.67239600$

$-3.12880100$

$-5.16438800$

$-1.87880600$

$-0.64574900$

$-3.26947900$

$-1.05287100$

$-6.02182200$

$-6.37989300$

$-6.86328600$

$-5.49371700$

$-2.51719400$

$-1.31097600$

$-3.32707000$

2. 77172800

1.52004500

1. 37871700
3.60119700

2.22866400

4.45878800

1.88641500

3.94104800

1.40651500

2.55788500

1.25814800

1.70841200

1. 61224600

2.22051600

2.09169400

3.99936400

2.60202000

5.53425200

1.99417500

4.61742100

1.14109900

0.63351500

1.50973000

1. 54727600

2.32934300

2.02729100

0.65099800

1.78999100

$-0.40628200$

2.80842600

0.64837600

0.77136200

1. 32528600

1.05021200

2. 28231000

0.02993800

1.53496000

0.22565500

0.42103700

0.93033100

0.13492100

$-1.05218300$

$-0.08871500$

$-1.90593600$

0.21931600

$-1.79305100$

$-0.42036600$

$-2.84911200$

1.24002800

$-2.59857500$

0.00480000

$-3.64235000$

1.66479400

$-2.91536600$

1.71485700

$-3.50165500$

1.06416500

$-3.23941800$

$-0.62723200$

$-4.35543400$

2. 47224200

$-4.12600700$

1.38573900

$-1.51320000$

$-1.14385300$

$-2.52312200$

$-0.91665100$

$-0.82215700$

$-1.18802200$

$-1.51251900$

$-2.10277300$

$-0.61239300$

$-1.32570400$

$-0.09140100$

$-1.11379700$

$-0.11621400$

$-2.08607100$

$-1.93398200$

0.63263100

$-2.61365900$

0.28126000

$-3.86889100$

$-0.11515500$ 


$$
0.30602300
$$

2. 45053900

2. 91222500

3. 22669400

1. 96802300

2.41389100

2.90189200

1. 58328300

3.23039700

0.06546000

3.77837000

4.04020500

3.24356100

5.03308400

6.15385700

5.08571900

7.30231000

6.12730700

6.23026200

4. 21820200

7. 34316500

8.16448600

6.25102900

8.23654700

$-0.56656000$

1.97396500

0.90915700

2. 51580700

1.99195500

2.76455700

2.50931700

3.83672800

2. 49769800
$-4.27175500$

$-4.91081000$

$-5.18149400$

$-4.57713900$

$-5.80812700$

$-0.46059000$

0.16908800

0.06542700

$-2.36129700$

$-1.34985500$

$-1.94784300$

$-2.98727900$

$-1.55519500$

$-1.14773100$

$-1.74853200$

0.21836600

$-1.00441900$

$-2.80961700$

0.96595800

0.70115700

0.35669900

$-1.48708000$

2.02561600

0.93798600

1.95411900

3.59650200

3.58023700

2. 57910900

1.71609700

4.86901500

5.31512700

4. 66012800

5.56905700
$-0.67953600$

0.12231300

$-0.83293000$

0.81395400

0.51910900

0.92799600

1. 84460200

0.02255400

1. 53030400

$-0.24924100$

$-0.55597400$

$-0.77760000$

$-1.42777600$

$-0.29356300$

0.29306200

$-0.60089400$

0.56689500

0.53408200

$-0.32659300$

$-1.04239700$

0.25696700

1.01961600

$-0.56711000$

0.46889100

$-1.30351900$

$-0.64751200$

$-1.28327600$

$-0.02824900$

$-0.03243700$

$-0.50289600$

0.46564600

$-0.50513700$

$-1.29540700$

\section{$o_{2}$-TS1-j}

C

C

C

C

C

C

$\mathrm{H}$

$\mathrm{H}$
0.11129500

1. 50020600

2.33276300

1.79056100

0.39592700

$-0.44330500$

$-0.53599100$

1.93694800
$-2.98168500$

3.95934500

$-3.10663800$

4.05532100

$-2.33426000$

3.24006700

$-1.41769300$

2.33088700

$-1.29068400$

2.25885400

$-2.07203200$

3.05353300

$-3.59784500$

4.57788800

$-3.81606600$

4.75403400 
3.41267300

$-0.03923300$

$-1.51941000$

2. 66928900

2.17842000

3. 64123400

2. 90135600

3.43677900

1.94553900

3.79147100

4.80069400

3.45971700

2.10891500

1.08040200

1.80325800

$-0.27977000$

0.47825300

2.61285700

$-0.54888600$

$-1.43568600$

0.24197000

$-1.57971200$

4. 34493000

4.21648100

5.37565900

4.10237300

1.38795700

0.56604700

2.43854800

$-4.05861800$

$-3.18475800$

$-3.55527800$

$-2.70702900$

$-4.98794700$

$-5.40807600$

$-5.62245800$

$-4.98207000$

$-3.14615300$

$-3.44841700$

$-2.04658800$

$-4.75924600$

$-1.28013200$

$-4.82553400$

$-5.42611200$
$-2.45317300$

3.29889200

$-0.60868100$

1.53458400

$-1.99276900$

2.92795400

$-0.60401200$

1.41068900

$-0.56706300$

0.43146800

$-1.08679700$

1.27350700

0.84023700

1.88983000

0.82378400

2.84850700

1.33516400

2.07244400

1.60896400

0.91182500

1.08407600

0.44192100

2. 91992300

0.62940300

3.38519800

0.75756400

2. 88257100

$-0.06283900$

4.28156500

1.78553500

3.17895300

0.22141300

4.62019500

2. 04673700

4.66018700

2. 40232800

4.04738700

1.29557000

3. 35867200

$-0.49999400$

5. 30181300

2.85911300

4.30209300

1.51532600

3.68364600

4.74902100

3.38685000

3.48076900

1.77615600

0.79329400

1.77509900

0.61649900

1.78494300

3.01160100

3. 88129000

3.48184100

3.63956000

2.78405100

4.44965800

$-0.59161200$

$-1.46302600$

$-0.60856200$

0.74061400

1.30085600

0.30737000

1.18314100
$-0.25508400$

$-0.04447300$

$-0.05900800$

$-1.30269200$

$-1.02653800$

$-1.02627700$

$-1.71514500$

0.25575500

0.09870000

$-0.22577100$

$-0.62785100$

$-0.09843200$

$-1.09719000$

0.45144200

0.41060400

0.56328600

1. 35226500

$-0.19236200$

1.08771400

$-0.27308800$

$-1.06417200$

$-1.32964400$ 


$\begin{array}{lrrr}\mathrm{H} & -4.06930700 & 0.18521900 & -1.84784400 \\ \mathrm{C} & -5.71380600 & -0.91146300 & -0.97680200 \\ \mathrm{C} & -7.05403300 & -0.78752200 & -0.58940400 \\ \mathrm{C} & -5.20841300 & -2.18872100 & -1.25347700 \\ \mathrm{C} & -7.87388400 & -1.91165200 & -0.48054300 \\ \mathrm{H} & -7.45968800 & 0.19974700 & -0.37546700 \\ \mathrm{C} & -6.02412000 & -3.31473000 & -1.14554000 \\ \mathrm{H} & -4.16629300 & -2.29943900 & -1.54283000 \\ \mathrm{C} & -7.35964900 & -3.17957900 & -0.75951300 \\ \mathrm{H} & -8.91263600 & -1.79726300 & -0.18156300 \\ \mathrm{H} & -5.61538100 & -4.29820100 & -1.36120200 \\ \mathrm{H} & -7.99589100 & -4.05678900 & -0.67710800 \\ \mathrm{Ag} & 3.15010200 & -0.28913600 & -1.97402200 \\ \mathrm{C} & 4.49345600 & -3.01326000 & -1.22912800 \\ \mathrm{O} & 4.35592600 & -2.13344600 & -2.09096500 \\ \mathrm{O} & 3.57200100 & -3.36971000 & -0.36841100 \\ \mathrm{H} & 2.66176800 & -2.93132500 & -0.52959800 \\ \mathrm{C} & 5.78982300 & -3.76269200 & -1.07458000 \\ \mathrm{H} & 6.28102200 & -3.42037500 & -0.15628200 \\ \mathrm{H} & 5.59980700 & -4.83417800 & -0.96891200 \\ \mathrm{H} & 6.44080200 & -3.56479600 & -1.92635700 \\ \mathrm{C} & 0.31734900 & -3.25729600 & -0.18888000 \\ \mathrm{O} & 1.14473400 & -2.48297200 & -0.69506000 \\ \mathrm{O} & -0.96189800 & -3.00492000 & -0.08574800 \\ \mathrm{H} & -1.22708900 & -2.04190600 & -0.23757900 \\ \mathrm{C} & 0.69785700 & -4.57998900 & 0.42579900 \\ \mathrm{H} & 0.89752400 & -4.39319600 & 1.48859600 \\ \mathrm{H} & -0.12848400 & -5.28976600 & 0.35445800 \\ \mathrm{H} & -60131600 & -4.98368100 & -0.03357100\end{array}$

\section{$o_{2}$-TS1-k}

$\mathrm{C}$
$\mathrm{C}$
$\mathrm{C}$
$\mathrm{C}$
$\mathrm{C}$
$\mathrm{C}$
$\mathrm{H}$
$\mathrm{H}$
$\mathrm{H}$
$\mathrm{H}$
$\mathrm{H}$
$\mathrm{C}$

$$
\begin{array}{r}
-0.17339900 \\
1.10345600 \\
2.22946800 \\
2.11341400 \\
0.81835400 \\
-0.31753400 \\
-1.05418400 \\
1.22350100 \\
3.21852600 \\
0.68152700 \\
-1.30622000 \\
3.37925000
\end{array}
$$$$
-3.57832300
$$$$
2.27546200
$$$$
-4.14075700
$$$$
2.18632700
$$$$
-3.32011400
$$$$
2.13159700
$$$$
-1.91880500
$$$$
2.17338700
$$$$
-1.37057100
$$$$
2.24656300
$$$$
-2.19113900
$$$$
\text { 2. } 29975800
$$$$
-4.21385200
$$$$
\text { 2. } 31566900
$$$$
-5.22057600
$$$$
2.15024900
$$$$
-3.76340200
$$$$
2.04743900
$$$$
-0.29300000
$$$$
2.27788300
$$$$
-1.74450700
$$$$
2.34084000
$$$$
-1.07661600
$$

2.16305600 
$\mathrm{H}$

$\mathrm{H}$

C

$\mathrm{H}$

$\mathrm{H}$

C

$\mathrm{O}$

$\mathrm{N}$

C

C

C

C

C

$\mathrm{H}$

C

$\mathrm{H}$

$\mathrm{H}$

$\mathrm{H}$

C

$\mathrm{H}$

$\mathrm{H}$

$\mathrm{H}$

C

O

O

C

$\mathrm{N}$

C

O

C

$\mathrm{H}$

$\mathrm{H}$

$\mathrm{H}$

C

O

O

$\mathrm{H}$

$\mathrm{Pd}$

C

$\mathrm{H}$

$\mathrm{H}$

C

C

C
4.12893100

3.79334400

3.19498900

2. 95579500

2.32997500

4.39867900

5.42406100

4.25280800

3.03732400

1.89666800

2.96234600

0.64057400

1.74849200

3. 85761200

0.59642800

$-0.48319600$

1. 69272700

$-0.35377700$

5.27049100

5.54823300

6.14102800

4.87864400

1.94922800

0.95155300

2. 87783300

$-3.53155400$

$-2.45367800$

$-2.59810100$

$-1.62921900$

$-3.89538400$

$-4.34944900$

$-4.61316000$

$-3.65747600$

$-2.86978300$

$-3.29989200$

$-1.85623500$

$-4.12150600$

$-0.72042000$

$-4.45427300$

$-4.87866700$

$-3.80888700$

$-5.56785500$

$-6.80771900$

$-5.37438300$
$-1.58139900$

$-1.04037300$

0.35037000

1.05988500

0.37614800

0.87164700

0.21329500

2. 12793300

2. 87412100

2.57690800

3. 84045400

3.14940300

4. 45546200

4.06055400

4.08315500

3.52621200

5.19398400

4.54132300

2.65061200

3.66906800

1.99797200

2. 65159700

1.40348900

0.57119500

1.21208900

1.43956200

2.38003900

3. 62453200

4.26864600

4.38684000

4.54500800

3.88268700

5.37118200

0.09528000

$-0.63054700$

$-0.22927600$

1.77930000

1.47957100

1.18444400

2.14167700

0.83183500

0.19987900

0.64606900

$-1.17485600$
1.55068400

3.17975400

1.63040600

2.43137800

0.96594000

0.83752300

0.68600800

0.28122900

0.41701800

$-0.35058100$

1.42606000

$-0.02194500$

1. 72305000

2.00044200

1.03124700

$-0.71227000$

2.51792600

1.28274300

$-0.63140000$

$-0.33872900$

$-0.57284200$

$-1.65264300$

$-1.28973100$

$-1.12317000$

$-2.07375400$

0.45341200

0.12674300

$-0.29666500$

$-0.82890400$

$-0.13363500$

$-1.11731900$

0.51606200

0.27894100

0.81913500

1.69410300

0.01578600

1.31066800

$-0.30141600$

$-0.77707600$

$-1.09582600$

$-1.58953200$

$-0.50557900$

$-0.03058700$

$-0.69502700$ 


$\begin{array}{lrrr}\mathrm{C} & -7.83374400 & -0.25735900 & 0.24960500 \\ \mathrm{H} & -6.97181300 & 1.71210500 & 0.11680200 \\ \mathrm{C} & -6.39711500 & -2.08064800 & -0.41497100 \\ \mathrm{H} & -4.41391900 & -1.54048400 & -1.04717500 \\ \mathrm{C} & -7.63018800 & -1.62547600 & 0.05700700 \\ \mathrm{H} & -8.79080600 & 0.10645400 & 0.61439300 \\ \mathrm{H} & -6.22785500 & -3.14399100 & -0.56246400 \\ \mathrm{H} & -8.42741300 & -2.33160900 & 0.27352500 \\ \mathrm{Ag} & 1.17777900 & -1.72792200 & -0.75215300 \\ \mathrm{C} & -1.44095200 & -3.67885000 & -0.87022300 \\ \mathrm{O} & -0.28200400 & -3.43022800 & -1.20981000 \\ \mathrm{O} & -2.27919600 & -2.82237500 & -0.33029500 \\ \mathrm{H} & -1.90496200 & -1.89829900 & -0.18770500 \\ \mathrm{C} & -2.03305400 & -5.05702700 & -1.02131500 \\ \mathrm{H} & -1.40394900 & -5.66615200 & -1.67094800 \\ \mathrm{H} & -2.08672800 & -5.52115000 & -0.02961900 \\ \mathrm{H} & -3.05199600 & -4.99481200 & -1.41273000 \\ \mathrm{C} & 4.57640400 & -1.70346300 & -1.44664700 \\ \mathrm{O} & 3.54380200 & -2.02098500 & -0.85403300 \\ \mathrm{O} & 4.66245100 & -0.72098300 & -2.32317500 \\ \mathrm{H} & 3.83768800 & -0.15933300 & -2.31796200 \\ \mathrm{C} & 5.88875200 & -2.39292900 & -1.19688400 \\ \mathrm{H} & 6.49468800 & -2.42740500 & -2.10543000 \\ \mathrm{H} & 6.41625200 & -1.79311000 & -0.44690400 \\ \mathrm{H} & 5.71634700 & -3.39569000 & -0.80280600\end{array}$

\section{m-TS5-cf1}

C

C

C

C

C

C

$\mathrm{H}$

$\mathrm{H}$

$\mathrm{H}$

$\mathrm{H}$

$\mathrm{H}$

C

$\mathrm{H}$

$\mathrm{H}$

C

$\mathrm{H}$
0.94836200

0.96152100

$-0.13982800$

$-1.23507700$

$-1.21394400$

$-0.12999300$

1.79398800

2.13046700

$-0.15353100$

$-2.06484200$

$-0.14007300$

$-2.45663100$

$-2.16423000$

$-3.02657000$

$-3.37375000$

$-4.31516800$
$-1.67686100$

$-1.69853200$

$-2.26897900$

$-2.79030900$

$-2.74159600$

$-2.20306100$

$-1.25445000$

$-2.13310300$

$-2.27883400$

$-3.13423900$

$-2.18782800$

$-3.29022600$

$-3.83358100$

$-3.97750700$

$-2.12051300$

$-2.52387800$
$-2.02728000$

$-0.61146600$

0.06566400

$-0.62728600$

$-2.02977800$

$-2.73037500$

$-2.56380500$

$-0.15341100$

1. 15175100

$-2.58345700$

$-3.81686600$

0.11339800

1.01699400

$-0.52245100$

0.53414200

0.92905300 
$-3.61800800$

$-2.72724700$

$-2.24272400$

$-2.68513800$

$-3.20290100$

$-2.37748200$

$-4.59214900$

$-2.98454700$

$-5.17743400$

$-5.20427600$

$-4.36697800$

$-2.33713800$

$-6.25795600$

$-4.80845300$

$-2.01223800$

$-2.58700500$

$-1.01226000$

$-1.93694500$

$-0.87312800$

$-0.31666400$

$-0.26225300$

1. 66201600

2.40876500

2.32424000

3. 26667100

2.64289600

3.77155100

4.00241500

4.08182200

3.66807400

5.57208100

5.89082700

6.12018000

5.78077500

3.33965900

1.80184500

1.08378700
$-1.50340400$

$-1.31823700$

$-1.89882900$

0.04910100

0.80133600

1.40338300

0.96128000

2.14893300

1.69080300

0.49949500

2.29202400

2. 60414500

1.79713000

2.87039900

0.75819500

0.64807000

0.34978300

1.81344100

1.28605200

0.60949800

1.84932700

0.12047000

3.08103100

2.06179500

4.24106600

5.13307700

4.00617500

4.45699200

$-1.53512000$

$-0.33439200$

$-1.74601400$

$-2.60427600$

$-0.84710500$

$-1.97008000$

$-2.56228100$

3.27101600

2. 56725900
$-0.33337900$

1.66559900

2.63596500

1. 57787100

0.47899700

$-0.49849500$

0.39960100

$-1.52141800$

$-0.63343300$

1.16861800

$-1.59775900$

$-2.26228800$

$-0.67617000$

$-2.40433900$

2.67219800

3. 59788200

2.82306000

2.40828000

$-0.54874300$

0.38825200

$-1.48778400$

0.19382600

0.19507600

0.89912900

0.62939400

0.74706000

1.56641000

$-0.15217400$

0.16142000

0.10924300

0.31650800

$-0.27953500$

0.03040500

1.36887500

0.11338400

$-0.94542700$

$-1.15950100$

\section{m-TS6}

C

C

C

C
0.54031100

0.69154200

1. 98888500

3.11214300
$-1.81872900$

2.11796900

$-1.97646300$

0.71824100

$-2.19423300$

$-2.22734000$

0.19924600

1.02793200 


$$
2.91757500
$$

1.64402800

$-0.44934600$

$-0.23927300$

2.12753600

3.78394700

1. 52746900

4.50482400

4.54126200

5.21948400

4.94729000

6.01684500

4.81959200

4.21506500

4.02810200

3.84179800

3. 92458400

2. 87479500

5.10598900

3.06546300

5.27330800

5.89433700

4.24358900

2. 25605900

6.19942500

4. 35842700

3.16920900

2.12284000

3. 67319400

3.22131500

1. 54489300

0.78757200

1.30878100

$-0.40538700$

$-3.33830900$

$-2.17389800$

$-2.25993700$

$-1.22873900$

$-3.58067600$

$-4.00402200$

$-3.40739000$

$-4.31339100$

$-2.92938300$

$-1.79144100$
$-2.07912300$

$-1.89608700$

$-1.64683900$

$-2.83005700$

$-2.28890700$

$-2.09728700$

$-1.78538900$

$-2.28048100$

$-2.97294100$

$-2.63154500$

$-0.88842500$

$-0.91531600$

$-0.14464700$

$-0.50187100$

$-1.34830000$

0.80780800

1.78949800

2.04007300

2. 53779300

3.03504900

3. 52465600

2. 32158200

3. 77340200

3.20217800

4.09160300

4.53813100

1.22446700

1.45932900

2.10522800

0.39760800

1.30186600

1.48595400

0.51619600

$-0.57928200$

$-0.76824100$

$-1.62577800$

$-2.93299500$

$-3.64119100$

$-3.67373000$

$-3.56252300$

$-4.73169700$

$-3.29161600$

0.37109100

0.83275300
2.41008400

2. 95865400

2. 53180100

0.25730900

$-0.87320100$

3.06886600

4.03333600

0.43806300

$-0.40826600$

1.19168900

$-0.06138200$

$-0.31105800$

0.72829700

$-1.35179700$

$-2.22565700$

$-1.50949000$

$-0.46772700$

0.44322500

$-0.39211400$

1. 41426600

0.57725200

$-1.10767100$

1.48656200

2.11656500

0.62138300

2.25008400

$-2.73972700$

$-2.52763400$

$-3.15381600$

$-3.44755300$

0.49569500

1. 45178600

$-0.51454900$

$-0.38728800$

$-0.57381200$

$-0.41184800$

$-0.25118900$

0.00478600

$-0.36532000$

$-1.36994200$

$-0.16814500$

0.35404100

$-1.49096600$

$-1.53013000$ 
$-3.92097500$

$-4.19408400$

$-3.76551400$

$-2.86404400$

$-4.07636300$

$-4.85864900$

$-4.54194200$

$-6.19876500$

$-5.54373500$

$-3.50461000$

$-7.20232800$

$-6.45702300$

$-6.87630000$

$-5.28343900$

$-8.23801300$

$-7.65739200$

$-3.57498200$
0.86247400

$-1.27987500$

$-0.17658100$

0.23967700

$-1.03185600$

0.86021400

2.22510000

0.47949500

3.18895300

2. 53101900

1.44006600

$-0.57781900$

2.79863700

4.24378800

1.12939700

3.54790600

1.63415300
$-2.22747700$

$-1.02551500$

0.81030300

1.27328800

1.42177300

0.72854200

0.74781600

0.58196100

0.62036100

0.86777600

0.45461400

0.57103900

0.47200700

0.64367600

0.34584900

0.37648200

$-2.71846900$

\section{m-TS7}

C

C

C

C

C

C

$\mathrm{H}$

$\mathrm{H}$

$\mathrm{H}$

$\mathrm{H}$

$\mathrm{H}$

C

$\mathrm{N}$

C

C

C

C

C

$\mathrm{H}$

C

$\mathrm{H}$

$\mathrm{H}$

$\mathrm{H}$

C
2.50255400

2.07956300

1.13804900

0.58532200

1.04042500

2.00469700

3. 23111100

3.12170500

0.77813600

0.62222700

2. 34425000

$-2.26822600$

$-2.86419400$

$-3.32072700$

$-2.49363000$

$-4.66951600$

$-3.04769700$

$-5.20979200$

$-5.28853600$

$-4.39684000$

$-2.39132100$

$-6.26250000$

$-4.80962100$

$-2.78269100$
1.73450900

1.04807200

1.67303900

2. 91398900

3. 57313200

3.00426600

1.27095800

0.43834000

1.14847900

4.54649600

3.54280700

1. 61696700

0.97196200

$-0.37897400$

$-1.50926000$

$-0.56003600$

$-2.79057200$

$-1.83949700$

0.32126400

$-2.96008900$

$-3.64541100$

$-1.95828900$

$-3.95980600$

1. 45368200
$-1.21792400$

$-0.05800600$

0.79531300

0.48701600

$-0.66810800$

$-1.50437500$

$-1.87812500$

0.54270500

1.67609000

$-0.91892900$

$-2.38526700$

0.82063600

$-0.24110700$

$-0.05177500$

$-0.22107800$

0.25959800

$-0.08532700$

0.39167900

0.39652400

0.21191600

$-0.20819500$

0.63313000

0.31124400

$-1.62536500$ 
H

$\mathrm{H}$

$\mathrm{H}$

C

O

O

C

O

O

C

$\mathrm{H}$

$\mathrm{H}$

$\mathrm{H}$

$\mathrm{Pd}$

C

C

O

$\mathrm{H}$

$\mathrm{H}$

$\mathrm{H}$

$\mathrm{H}$
$-3.34569900$

$-1.74228000$

$-3.22949800$

$-1.02938600$

$-0.47803500$

$-0.29231200$

4.17713100

3.22477600

4.18944700

5.38244600

6.29745700

5.37201800

5.35637500

1.43678700

$-1.92131600$

$-0.56935600$

$-2.09346800$

$-1.92496000$

$-2.72517200$

$-0.55765400$

$-0.49818900$
2.38338200

1.59507900

0.69624200

$-1.42012700$

$-0.26786700$

$-2.44917400$

$-1.33091000$

$-1.88939900$

$-0.11714900$

$-2.17970000$

$-1.62520500$

$-2.38438400$

$-3.12245300$

$-0.93925500$

3.10108700

3.46833000

1.06676500

3.42063400

3.65303500

3.06733300

4.56063500
$-1.76083800$

$-1.93368200$

$-2.27154600$

$-0.46158900$

$-0.65060100$

$-0.43210100$

0.61448500

$-0.01088800$

0.99268700

0.95235800

0.72849500

2.02869500

0.40472300

$-0.34667400$

0.64299600

1.29206600

1.90162400

$-0.40140300$

1.14914400

2.30953800

1. 35958800

\section{$m$-TS8}

C

C

C

C

C

C

$\mathrm{H}$

$\mathrm{H}$

$\mathrm{H}$

H

$\mathrm{H}$

C

$\mathrm{H}$

$\mathrm{H}$

C

$\mathrm{H}$

$\mathrm{H}$

C

O

N
$-2.16545200$

$-1.42036000$

$-0.33095700$

0.03561500

$-0.73224700$

$-1.83312300$

$-2.99782000$

$-1.67870900$

0.25034000

$-0.47322500$

$-1.81814300$

1.24656200

1.01722500

1.54133500

2.42944200

2.71447600

2.13223700

3.63148700

3.61829200

4.75094600
0.35865200

$-0.28790500$

$-1.08431200$

$-1.25149500$

$-0.60817700$

0.20823600

0.99980000

$-0.17614400$

$-1.58615800$

$-0.74147100$

1.39070200

$-2.06629600$

$-2.70208200$

$-2.74266700$

$-1.15845200$

$-0.53159200$

$-0.46608800$

$-1.96128400$

$-3.18270100$

$-1.22417200$
2.45532800

3.43632600

3.06817000

1.72423600

0.75010700

1.08777700

2.73421900

4.48612700

3.83904500

$-0.29797900$

0.49555600

1.33036300

0.46886700

2.13920500

0.96531600

1.82006600

0.16966200

0.48000100

0.41824400

0.13151000 
4.75705400

4.30391600

5.29584200

4.40417300

5.37913600

5.64765000

4.93209400

4.05311000

5.79342200

4.99227600

5.86195300

6.81242000

5.80495900

5.79062300

3.68335300

3.26201000

3.60860400

3.15659300

$-3.11586200$

$-4.02287700$

$-1.88122000$

$-3.55460300$

$-4.61572300$

$-2.94848800$

$-3.39146100$

$-4.39587200$

$-3.31763500$

$-5.02684700$

$-4.89382100$

$-5.47168300$

$-5.54303900$

$-4.05037200$

$-3.50015700$
0.20419400

0.95322700

0.87625300

2. 35345700

2. 26834900

0.28146700

3. 00928600

2. 92404500

2.77024500

4.09350900

$-1.89802300$

$-1.48387600$

$-2.95945600$

$-1.77670600$

0.28619400

$-0.85323700$

1.10768600

0.59090800

2.92039000

2.02938800

2. 68405800

4.35810000

4.46473300

5.01173000

4.64688200

$-1.97206800$

$-1.97065800$

$-0.88070800$

$-3.25812900$

$-3.80091700$

$-3.05030000$

$-3.88631500$

0.05059000
0.09964700

$-1.01129800$

1.20041200

$-0.97919900$

1.22496900

2.03791300

0.13009900

$-1.83111200$

2.09485800

0.13880000

$-0.53845000$

$-0.18769300$

$-0.29749600$

$-1.62669500$

$-2.19091600$

$-2.22890500$

$-3.27006000$

$-3.96315100$

0.00406400

$-0.04843300$

0.17000900

$-0.16678900$

0.06396900

0.46440400

$-1.21153200$

$-1.31307500$

$-0.61201000$

$-1.45949100$

$-1.90819400$

$-1.15088800$

$-2.76155800$

$-2.20606200$

$-0.19972400$

\section{m-TS5-cf2}

C

C

C

C

C

C

$\mathrm{H}$

$\mathrm{H}$
1.36970100

1.20780900

0.05114600

$-0.92519400$

$-0.73198400$

0.40488000

2.29860500

$-0.08587400$
$-2.51415000$

$-1.81100800$

$-2.05879200$

$-2.96899300$

$-3.63342400$

$-3.42043200$

$-1.87756500$

$-1.52377000$
$-1.59327800$

$-0.37476700$

0.40339900

$-0.00923400$

$-1.23095600$

$-2.01669600$

0.35019600

1.33995000 
C

$\mathrm{H}$

$\mathrm{H}$

C

$\mathrm{H}$

$\mathrm{H}$

C

$\mathrm{O}$

$\mathrm{N}$

C

C

C

C

C

$\mathrm{H}$

C

H

$\mathrm{H}$

$\mathrm{H}$

C

$\mathrm{H}$

H

$\mathrm{H}$

C

O

O

$\mathrm{H}$

H

$\mathrm{H}$

$\mathrm{Pd}$

C

O

O

C

$\mathrm{H}$

$\mathrm{H}$

$\mathrm{H}$

$\mathrm{H}$

C

O

O

C

$\mathrm{H}$

$\mathrm{H}$

$$
\begin{aligned}
& -2.18817400 \\
& -2.70190100 \\
& -1.94856200 \\
& -3.15187400 \\
& -3.17604000 \\
& -4.17355100 \\
& -2.85449300 \\
& -2.39117700 \\
& -3.26065400 \\
& -3.47633300 \\
& -2.40147500 \\
& -4.78858700 \\
& -2.67450600 \\
& -5.04719600 \\
& -5.60130000 \\
& -3.98619000 \\
& -1.83976900 \\
& -6.07292800 \\
& -4.17921700 \\
& -3.18396900 \\
& -4.07422100 \\
& -2.28488500 \\
& -3.14390400 \\
& -0.96563200 \\
& -0.15362000 \\
& -0.64196600 \\
& -1.48781300 \\
& 0.52772300 \\
& 2.26591300 \\
& 1.77683100 \\
& 2.10970000 \\
& 2.30088800 \\
& 1.27294300 \\
& 2.86401900 \\
& 2.15715000 \\
& 3.58277800 \\
& 3.37887700 \\
& 0.64444900 \\
& 4.16263900 \\
& 3.74815700 \\
& 3.46458200 \\
& 5.60090800 \\
& 6.13135800 \\
& 6.09408600
\end{aligned}
$$

$-3.19068300$

$-4.08213900$

$-3.36921900$

$-1.99021200$

$-1.57308700$

$-2.33031000$

$-0.92766500$

$-1.24745900$

0.35988600

0.82411400

1.07656500

1.00334800

1.45662000

1.41909300

0.79275500

1.63862800

1.61193200

1.55401100

1.94739000

1.37759500

2.01320100

1.98483000

0.86808300

0.97412200

0.54294700

1.32385000

$-4.33928500$

$-3.96309500$

$-2.35414100$

0.21614600

3.15868200

2. 31383700

3. 03476000

4.46218700

5.29255800

4.47913000

4.58302400

2.23475600

$-1.01889200$

$-0.01429700$

$-2.03211200$

$-0.98951900$

$-0.13523500$

$-1.92459100$
0.79602600

0.41946600

1.84932200

0.72599500

$-0.28298700$

0.94641600

1.79351000

2.88345700

1.51726500

0.18051400

$-0.69706800$

$-0.26567700$

$-2.01791100$

$-1.57195600$

0.42349900

$-2.45288300$

$-2.69332500$

$-1.90384200$

$-3.47635700$

2.56875900

2. 51713800

2.43972600

3.53149700

$-0.25592400$

$-1.15158000$

0.90204100

$-1.57002000$

$-2.95027400$

$-2.18737600$

$-0.52731800$

0.17735900

$-0.71098300$

1.17475500

0.17490200

0.07631500

$-0.64428900$

1.13347400

1.07878900

0.66852000

0.00737800

0.96620100

1.14014500

0.71795700

0.86010400 


\section{m-TS5-cf3}

C

C

C

C

C

$\mathrm{H}$

$\mathrm{H}$

$\mathrm{H}$

$\mathrm{H}$

C

$\mathrm{H}$

$\mathrm{H}$

C

$\mathrm{H}$

$\mathrm{H}$

C

$\mathrm{O}$

N

C

C

C

C

C

$\mathrm{H}$

C

$\mathrm{H}$

$\mathrm{H}$

$\mathrm{H}$

C

$\mathrm{H}$

$\mathrm{H}$

$\mathrm{H}$

C

O

O

$\mathrm{H}$

$\mathrm{Pd}$

C

O
1.44142200

0.51979500

$-0.67367600$

$-0.95048900$

$-0.00807600$

1.19531300

2.36778700

0.71134800

$-1.42397700$

2.33004000

$-2.29777600$

$-2.17484700$

$-2.80547000$

$-3.18787700$

$-2.74117400$

$-4.17090400$

$-3.39507000$

$-3.52093400$

$-3.39088200$

$-3.46445600$

$-2.30706000$

$-4.71190000$

$-2.41720300$

$-4.81715500$

$-5.59401500$

$-3.66498700$

$-1.51250700$

$-5.79229500$

$-3.73598900$

$-3.50998100$

$-2.76190900$

$-3.32908900$

$-4.51423600$

$-0.93948200$

$-0.09887900$

$-0.69875900$

$-0.22121400$

1.80059100

2.23788300

2.40830000
$-2.28775700$

$-3.15083900$

$-3.44080200$

$-2.90708100$

$-2.05848800$

$-1.72321000$

$-2.05005700$

$-3.58146900$

$-4.06191400$

$-1.82986300$

$-3.13281400$

$-3.36212900$

$-3.97994300$

$-1.87912500$

$-1.04768900$

$-2.07009800$

$-1.52972900$

$-2.41578700$

$-0.19437200$

0.84699400

1. 49442400

1. 20240500

2. 46174800

2.19045100

0.68351800

2. 82024200

2. 92565900

2. 45742800

3.58143200

0.20743900

0.97537200

$-0.67175500$

0.59685600

1.16470100

0.83154600

1.27334400

$-1.60779400$

0.28512500

2. 99398500

2. 35823900
1.01814900

1.59966300

0.93343700

$-0.33549900$

$-0.91796000$

$-0.25818300$

1.53466600

2.57875400

1. 41217400

$-0.98000000$

$-0.98943500$

$-2.05483600$

$-0.52056000$

$-0.85694100$

$-1.40681000$

$-1.30721600$

0.61917100

1. 46048200

0.95556500

$-0.02396600$

$-0.48624400$

$-0.54711800$

$-1.49239300$

$-1.52626800$

$-0.18248400$

$-2.00124300$

$-1.87309300$

$-1.92382600$

$-2.77305700$

2. 35880900

2. 56612100

2.97704500

2. 56954100

0.05158700

$-0.86040600$

1.27131300

$-1.88352600$

$-0.33486900$

0.97591600

$-0.07615200$ 


\section{m-TS5-cf4}

C

C

C

C

C

C

$\mathrm{H}$

H

$\mathrm{H}$

H

$\mathrm{H}$

C

$\mathrm{H}$

$\mathrm{H}$

C

$\mathrm{H}$

$\mathrm{H}$

C

O

N

C

C

C

C

C

$\mathrm{H}$

C

$\mathrm{H}$
1.36843500

3.06559500

2. 40909100

3.78898300

3.58283300

0.68533200

4.26438200

3.78364200

3.57315700

5.76878100

6.03849800

6.22241100

6.14268100
2.70550800

4.21535100

5.08418700

4.38724400

4.07100700

1.99578600

$-1.18727900$

$-0.21579700$

$-2.02642000$

$-1.33763800$

$-2.39519000$

$-0.78025700$

$-0.93840900$
1.91029300

1.27936300

1.39167500

0.48237700

2.23359500

1.64311700

$-0.69798000$

$-0.03072200$

$-1.35463200$

$-0.71862000$

$-0.66940600$

0.10230200

$-1.66840400$

\subsection{0}

1.00200500

$-0.19042600$

$-1.14648300$

$-0.89726300$

0.28102200

2.14002800

2.10100000

$-0.36788400$

$-1.65631700$

0.44677500

$-2.48666800$

$-3.05229900$

$-2.35501600$

$-3.31190300$

$-4.34662800$

$-2.90807600$

$-3.29431800$

$-3.43816300$

$-2.99165500$

$-3.24779600$

$-2.23468500$

$-4.58313400$

$-2.59991300$

$-4.92807600$

$-5.34733900$

$-3.92780300$

$-1.81248300$
$-2.82944600$

$-1.82799600$

$-1.87775100$

$-2.87213700$

$-3.83868200$

$-3.83412600$

$-2.81559000$

$-1.70428900$

$-1.10335700$

$-4.58930300$

$-4.60106500$

$-2.83082000$

$-3.73460700$

$-2.80433800$

$-1.59196100$

$-1.70092200$

$-0.69110600$

$-1.48038500$

$-2.47660800$

$-0.24521800$

0.96789200

1.74958900

1.37886300

2.92368500

2.55278900

0.75671600

3.33283300

3. 49911900
0.72256200

$-0.25492100$

$-1.01492000$

$-0.81247500$

0.17523900

0.92570800

1.30181900

$-0.99730300$

$-1.75787200$

0.37366500

1.67744200

$-1.51969200$

$-1.27734100$

$-2.60838400$

$-1.09243400$

$-1.43961800$

$-1.56012500$

0.43251500

1.13583400

0.95851400

0.24343200

$-0.34893100$

0.14027200

$-1.02651000$

$-0.52695500$

0.59770300

$-1.10937900$

$-1.50016100$ 
$-5.97025900$

$-4.18090100$

$-2.72775600$

$-2.43342600$

$-1.91239500$

$-3.61977300$

$-0.77162400$

$-0.34137800$

$-0.06773200$

1. 63720500

4.05690900

3.30976400

5.55338900

6.08765500

5.84400500

5.81042500

2.56134000

2.33119400

2.06813200

3. 64151100

3.46692800

4.26054100

2.89682400

3.89656700

1.31994700
2.85311500

4.24949800

$-0.11957200$

$-1.09902900$

0.59284500

0.22010000

1. 39047800

0.61855300

1.87498600

0.06678700

$-1.12295900$

$-1.75462400$

$-1.22942500$

$-0.67949100$

$-2.28427100$

$-0.83015000$

2.97612800

1.97895300

3.19016500

$-0.40919600$

4.07413300

4.24542800

5.00483900

3.80268600

2.53289500
$-0.59197800$

$-1.63438700$

2.39106300

2. 76805400

2.53154100

2. 93346700

$-0.36786500$

0.56146900

$-1.28818800$

0.38975700

$-0.71487900$

$-1.52238100$

$-0.91065600$

$-0.13513800$

$-0.89810100$

$-1.89716700$

0.46818100

1.16919500

$-0.72416500$

0.25273400

0.96092000

0.22654300

1. 04943500

1.92515900

$-0.96327900$

\section{m-TS5-cf5}

C

C

C

C

C

C

$\mathrm{H}$

$\mathrm{H}$

$\mathrm{H}$

C

$\mathrm{H}$

$\mathrm{H}$

C

$\mathrm{H}$

$\mathrm{H}$

C
$-1.53983800$

$-1.10675300$

0.10937300

0.86814500

0.40902100

$-0.78414300$

$-2.10738300$

0.46039500

0.99804500

2.19880100

2.38949300

2.19982500

3. 35502800

3. 34833100

4.30736500

3.31777700
$-2.71518700$

$-1.86761100$

$-2.16786400$

$-3.28599600$

$-4.09672400$

$-3.82095700$

$-1.64869300$

$-1.51828700$

$-4.96494700$

$-3.57481100$

$-4.65442100$

$-3.23720300$

$-2.85738400$

$-3.10963700$

$-3.20239600$

$-1.35450500$
0.99990900

$-0.04894500$

$-0.70307100$

$-0.34860300$

0.70247800

1.37504200

$-0.87152500$

$-1.50015900$

0.99241000

$-1.00539900$

$-1.00647600$

$-2.04674300$

$-0.27449600$

0.78963600

$-0.69233900$

$-0.55484900$ 


\begin{tabular}{|c|c|c|c|}
\hline O & 3.50367400 & -0.95499900 & -1.70014100 \\
\hline $\mathrm{N}$ & 3.09574300 & -0.48967500 & 0.48985800 \\
\hline $\mathrm{C}$ & 3.34214100 & 0.90320900 & 0.24111600 \\
\hline $\mathrm{C}$ & 2.33382500 & 1.78811900 & -0.17627300 \\
\hline $\mathrm{C}$ & 4.65242900 & 1.36663300 & 0.38549300 \\
\hline C & 2.67085800 & 3.12105900 & -0.45159000 \\
\hline C & 4.97107500 & 2.70044300 & 0.13547000 \\
\hline $\mathrm{H}$ & 5.42101600 & 0.65926600 & 0.68261600 \\
\hline C & 3.97422100 & 3.58195500 & -0.28669700 \\
\hline $\mathrm{H}$ & 1.88752200 & 3.78323300 & -0.804821 \\
\hline $\mathrm{H}$ & 5.99430200 & 3.04482600 & 0.2576390 \\
\hline $\mathrm{H}$ & 4.21261600 & 4.62094900 & -0.49603600 \\
\hline $\mathrm{C}$ & 2.67054400 & -0.88344800 & 1.8343010 \\
\hline $\mathrm{H}$ & 2.18530700 & -0.02513400 & 2.2984090 \\
\hline $\mathrm{H}$ & 3.51758700 & -1.20227400 & 2.4568590 \\
\hline $\mathrm{H}$ & 1.92723700 & -1.68122700 & 1.7836330 \\
\hline $\mathrm{C}$ & 0.89227800 & 1.40544900 & -0.3488430 \\
\hline O & 0.42919200 & 0.55168700 & 0.4972490 \\
\hline O & 0.23379100 & 1.97010200 & -1.2506310 \\
\hline $\mathrm{Pd}$ & -1.57278900 & 0.14956500 & 0.360368 \\
\hline $\mathrm{C}$ & -2.41963300 & 3.08320700 & 0.2982350 \\
\hline O & -2.15075200 & 2.13089000 & 1.0492460 \\
\hline O & -1.95197900 & 3.23491000 & -0.911343 \\
\hline $\mathrm{C}$ & -3.34107400 & 4.18507400 & 0.7494460 \\
\hline $\mathrm{H}$ & -2.79444500 & 5.13363000 & 0.7682680 \\
\hline $\mathrm{H}$ & -3.74158100 & 3.96014800 & 1.7379010 \\
\hline $\mathrm{H}$ & -4.15590500 & 4.29185700 & 0.0260070 \\
\hline $\mathrm{H}$ & -1.17114300 & 2.59167000 & -1.0911770 \\
\hline $\mathrm{C}$ & -4.04309700 & -0.90944100 & -0.731014 \\
\hline O & -3.60738400 & -0.18459100 & 0.224238 \\
\hline O & -3.31953600 & -1.63304600 & -1.475463 \\
\hline C & -5.53340900 & -0.87657800 & -0.991775 \\
\hline $\mathrm{H}$ & -6.07063800 & -0.51574600 & -0.113193 \\
\hline $\mathrm{H}$ & -5.88276500 & -1.86969500 & -1.283045 \\
\hline $\mathrm{H}$ & -5.72215700 & -0.19368100 & -1.8281390 \\
\hline $\mathrm{H}$ & -1.11960800 & -4.47267000 & 2.1773030 \\
\hline & -2.48675200 & -2.50930600 & 1.4921840 \\
\hline
\end{tabular}

\section{m-TS5-cf6}

$\mathrm{C}$
$\mathrm{C}$
$\mathrm{C}$
$\mathrm{C}$

$$
\begin{array}{r}
0.57529800 \\
-0.61651600 \\
-1.44024700 \\
-1.07402300
\end{array}
$$$$
-1.48196200
$$$$
1.98934100
$$$$
-2.01863200
$$$$
2.46256900
$$$$
-2.74740900
$$$$
\text { 1. } 59942600
$$$$
-2.98982100
$$$$
0.26578800
$$ 
0.13189500

0.97325400

1.20576400

$-0.92598600$

$-2.40027900$

0.42766500

2. 24681300

$-2.03417800$

$-1.52674100$

$-2.84622100$

$-2.64149400$

$-1.88102900$

$-3.45708000$

$-3.21352500$

$-3.94644000$

$-2.81172200$

$-3.20735700$

$-2.28474700$

$-4.56904800$

$-2.76911200$

$-5.02957900$

$-5.26134400$

$-4.12216700$

$-2.04995100$

$-6.09148900$

$-4.46783400$

$-2.11573000$

$-2.01545800$

$-2.69604000$

$-1.11039000$

$-0.80079100$

$-0.30061500$

$-0.13651600$

1. 68264000

2.48290800

2. 32427500

1. 94589700

3.35468300

4.13375800

2.75445900

3.80592700

1. 21090100

4.16841400

3.69775100
$-2.45938200$

$-1.68868100$

$-0.89232100$

$-1.85108300$

$-3.11225600$

$-2.62326500$

$-2.08045100$

$-3.67424600$

$-4.46249800$

$-4.13883800$

$-2.65158900$

$-2.34173300$

$-3.12187200$

$-1.48254400$

$-1.70663000$

$-0.21235900$

0.87181500

1.61336900

1.18776400

2.67281100

2. 23664100

0.58598500

2.98929200

3. 23126600

2. 46517900

3. 81342500

0.10407700

1.18756200

$-0.24258500$

$-0.31909100$

1. 35785500

0.56938300

1.93975500

0.08130900

3. 01514300

1.97141800

3.27149100

4.12489400

4. 35087700

5.03134200

3.83127100

2.60286600

$-1.47462000$

$-0.34696400$
$-0.18993800$

0.64594000

2.64909200

3.49045400

1.95185800

$-1.22475900$

0.56165600

$-0.68571500$

$-1.25510100$

$-0.12067200$

$-1.67749400$

$-2.39679600$

$-2.24044800$

$-0.87490400$

0.08465700

$-1.21799000$

$-0.36615600$

0.40463800

$-0.30579600$

1.19124400

0.48474200

$-0.88420800$

1.23326800

1.77936200

0.51368900

1.85095800

$-2.47065900$

$-2.53602200$

$-3.33393600$

$-2.49553500$

0.47695700

$-0.40479100$

1.36888900

$-0.24595200$

$-0.44658600$

$-1.10085800$

0.71533200

$-0.97462800$

$-0.23929900$

$-1.10416900$

$-1.92240700$

0.98638700

0.13096800

$-0.20873600$ 


\section{m-TS5-cf7}

C

C

C

C

C

C

$\mathrm{H}$

$\mathrm{H}$

H

$\mathrm{H}$

$\mathrm{H}$

C

$\mathrm{H}$

$\mathrm{H}$

C

$\mathrm{H}$

$\mathrm{H}$

C

$\mathrm{O}$

N

C

C

C

C

C

$\mathrm{H}$

C

$\mathrm{H}$

$\mathrm{H}$

$\mathrm{H}$

C

$\mathrm{H}$

$\mathrm{H}$

$\mathrm{H}$

C

o
3.47815200

5.66979400

6.15052800

5.90275000

6.04549800
$-2.46487000$

$-1.65344900$

$-0.76411200$

$-2.52782000$

$-1.85217500$
0.52532100

0.06978000

$-0.33902900$

$-0.54536300$

1.07880000
(

.

(

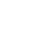

.

C

$-1.42169000$

$-0.61878000$

0.55305000

0.95455300

0.15270300

$-1.05525100$

$-2.35787300$

$-0.90400200$

1.17397500

0.46067500

$-2.06693600$

2.26498300

2.24410800

2.43154800

3. 46329800

4.38943200

3.48667300

3.45385700

3.71284400

3.15792700

3. 35250300

2. 31579600

4.61969800

2.57654800

4.85664900

5.41224700

3.83680800

1.77152900

5.84419800

4.02345400

2.72795900

1.88615600

3.55015000

2.39989900

0.90195200

0.37227400
$-2.37833600$

$-3.33106900$

$-3.75790500$

$-3.23892400$

$-2.26764500$

$-1.83693300$

$-2.06976400$

$-3.75262000$

$-4.51256200$

$-1.83263800$

$-1.90758900$

$-3.67010900$

$-3.52371300$

$-4.73818300$

$-2.87438400$

$-3.31132400$

$-2.95580100$

$-1.43280600$

$-1.21935200$

$-0.41942000$

0.93956900

1.87793300

1.35832200

3.22973900

2. 69634100

0.62306100

3.64048900

3.94734400

3.00443800

4.68872400

$-0.66282700$

$-1.35710100$

$-1.05074300$

0.28097000

1.50380200

0.63751500
1.29782400

1. 91791600

1.28780800

0.04469400

$-0.55454700$

0.04217700

1.75519700

2.87793300

1.76732200

$-1.50088900$

$-0.76285100$

$-0.57538800$

$-1.66021000$

$-0.39039400$

$-0.00940200$

$-0.39722000$

1.08071500

$-0.52334400$

$-1.70199800$

0.36404800

$-0.04771300$

0.05676100

$-0.46744800$

$-0.20049700$

$-0.77318300$

$-0.55223100$

$-0.62670100$

$-0.07404900$

$-1.10520900$

$-0.84205100$

1.74757900

1.78261700

2. 36230500

2.18339800

0.40975300

$-0.37477700$ 


\section{$m$-TS5-cf8}

C

C

C

C

C

C

$\mathrm{H}$

$\mathrm{H}$

$\mathrm{H}$

C

$\mathrm{H}$

$\mathrm{H}$

C

$\mathrm{H}$

$\mathrm{H}$

C

O

$\mathrm{N}$

C

C

C

C

C

$\mathrm{H}$
0.35800700

$-1.60938100$

$-4.05055900$

$-3.31037400$

$-5.54845800$

$-5.84250400$

$-6.07186100$

$-5.81889800$

$-2.56473900$

$-2.29168500$

$-1.97934600$

$-3.63304400$

$-4.39771500$

$-3.20148300$

$-4.08195600$

$-1.11228500$

$-3.62666000$
2.07504700

0.19195300

$-1.24159000$

$-2.12918500$

$-1.33943800$

$-2.37738900$

$-0.91660200$

$-0.75373300$

3.02833300

2. 23080100

3. 04806900

4.07320200

3.93727400

5.06955600

3.98625700

2. 49365200

$-0.23789400$
1.37960500

$-0.20947900$

$-0.81835800$

$-1.32880600$

$-1.01306700$

$-1.17828600$

$-0.15289500$

$-1.89930200$

0.39348000

$-0.51989800$

1.55955100

0.21263900

0.98489000

0.35108000

$-0.77680700$

1.53494400

$-0.15195600$
$-1.04546500$

$-1.35412600$

$-0.57147300$

0.49577600

0.76452100

$-0.00730100$

$-2.64496400$

$-0.80276200$

1. 61356000

1.39518900

2. 11424500

0.80055900

2.16276400

2.82065300

1.46387700

2.96894100

3.16169600

3. 41070300

3.57911300

2. 45555900

4.86058300

2.65236900

5.04277300

5.71099900
$-2.21343600$

$-1.69203400$

$-2.11461300$

$-2.99705700$

$-3.49069200$

$-3.12451700$

$-1.82618600$

$-1.71670700$

$-4.15343300$

$-3.35083500$

$-4.11352600$

$-3.77903000$

$-2.13251100$

$-2.44805000$

$-1.39927300$

$-1.45159900$

$-1.99770100$

$-0.16416200$

0.66506600

1.08923900

1.01880500

1.83253600

1.79468700

0.65818300
$-1.84011400$

$-0.56169700$

0.54391400

0.39608800

$-0.89078400$

$-1.99606400$

$-0.30280700$

1. 52917200

$-1.02725800$

1.56706200

1.25405300

2.38434400

2.11927600

2. 94029800

2.53793700

1.01443800

$-0.06607500$

1.25088200

0.09278500

$-0.64674400$

$-0.32526400$

$-1.81622100$

$-1.47162100$

0.24574600 


\section{p-TS5-cf1}

$\mathrm{C}$
$\mathrm{C}$
$\mathrm{C}$
$\mathrm{C}$
$\mathrm{C}$
$\mathrm{C}$
$\mathrm{H}$
$\mathrm{H}$
$\mathrm{H}$
$\mathrm{H}$
$\mathrm{H}$
$\mathrm{C}$
$\mathrm{H}$

3.93664600

1.78312800

6.04655000

4.07215000

3. 34813200

2. 36824800

3.57160900

4.11568800

1.04566800

0.18621800

0.77092200

$-1.76094800$

$-4.39386500$

$-3.90827400$

$-5.86051600$

$-6.05866500$

$-6.14390300$

$-6.45644300$

$-1.75726300$

$-2.04104300$

$-0.94771600$

$-2.34644800$

$-2.82761400$

$-1.54428800$

$-3.06919300$

$-0.41349400$

$-3.74867700$

0.22807000

$-1.63381000$
2.19488300

2.13022600

2.06611900

2.78440300

0.49350100

0.94968300

$-0.22390800$

1.27244200

0.83331300

0.62918200

0.89243500

0.36830500

$-0.81780200$

$-1.97789500$

$-0.71926800$

$-1.34211200$

0.31550400

$-1.11777200$

3.14071900

2. 51820700

2.72241300

4.50027600

4.49849500

5.24519400

4.75675900

1.89267200

0.26808000

$-3.52978500$

$-1.90252500$
$-2.22258700$

$-2.39350700$

$-1.78687300$

$-3.12504300$

2. 55472300

2. 72512700

3.34838500

2. 58296900

$-0.18398300$

$-1.11200300$

1.04185200

$-0.50045600$

0.15171900

$-0.01197600$

0.51372200

1.39104300

0.70935900

$-0.31395900$

0.73729700

$-0.29634300$

1.67867400

1.00718100

1.99041000

1.03653900

0.23266700

1.41653300

0.02919900

$-2.97637000$

$-2.69938200$
1.08729800

0.54869600

$-0.73105600$

$-1.52116000$

$-0.97710400$

0.29454900

2.36763100

$-1.15550700$

$-1.58142000$

0.68017300

$-2.97633900$

$-3.14132000$
$-1.73957400$

$-2.02030300$

$-2.54029000$

$-2.79892500$

$-2.58818400$

$-2.05169200$

$-2.17385600$

$-2.69526300$

$-2.80639800$

$-1.84321900$

$-3.16722800$

$-3.71902000$
$-0.43788200$

0.84246000

0.98922700

$-0.14244100$

$-1.42140200$

$-1.56813600$

$-0.53985600$

1.97655700

$-2.29935700$

$-2.56241200$

0.01555700

0.94538600 


\begin{tabular}{|c|c|c|}
\hline 100 & -3.7938 & -0.82046900 \\
\hline-3.83976900 & -1.88390000 & 0.05291400 \\
\hline 4.90047300 & -2.16319700 & 0.07882600 \\
\hline 3.67 & -1.29810400 & -0.8530580 \\
\hline 3 . & -1.09184400 & 1.3 \\
\hline 3 . & -1.65769600 & 2.4 \\
\hline 3.2 & 0.24462100 & 1.2 \\
\hline 3 . & 0.98062700 & 0.0 \\
\hline 2 . & 1.35480800 & -0.6 \\
\hline 4 . & 1.25 & -0.5 \\
\hline 2.2 & 2.0 & -1.9 \\
\hline 4.6 & 1.96948900 & $-1 \cdot 7$ \\
\hline 5.4 & 0.9 & \\
\hline-3.5 & 2.32 & -2.4 \\
\hline 1.3 & 2.26 & -2.4 \\
\hline 5 . & 4600 & -2.1 \\
\hline-3.5 & 2.84 & $-3 \cdot 3$ \\
\hline-3.0 & 0.9 & 2.4 \\
\hline-3.9 & $0.9^{\circ}$ & 3.1 \\
\hline-2 . & 0.45 & \\
\hline-2.6 & 1.97381300 & 2.25 \\
\hline-0.7 & 1.0 & -0.1 \\
\hline 0 . & & -1 \\
\hline-0.51 & 1.20733200 & 1.0 \\
\hline 1.9 & 0.16584200 & -0.4 \\
\hline 1.1 & -1.8 & 1.7 \\
\hline 4 . & -1.611 & -0.0 \\
\hline 3.86 & -0.42656600 & 0.0 \\
\hline 3.5 & -2.58677300 & -0.4 \\
\hline 5 . & -1.8 & \\
\hline 5.82 & -2.68729500 & 0.9 \\
\hline 6.21 & -2.25321500 & -0.7294 \\
\hline 6.25 & -0.99 & 0 . \\
\hline 2.46 & 2.90183200 & 0.7865 \\
\hline 2.70 & 2.19245600 & -0.202 \\
\hline 1.52052 & 2.69518800 & 1.6657 \\
\hline 0.84708 & 1.96961600 & 1.40811 \\
\hline 3.293026 & 4.12979200 & 1.06893 \\
\hline 2.65 & 5.01680900 & 1.0257 \\
\hline 3.69 & 4.068237 & 2. \\
\hline 4.10 & 4.2142700 & 0.3 \\
\hline
\end{tabular}




\begin{tabular}{|c|c|c|c|}
\hline C & 0.93182600 & -1.78402700 & -0.47397200 \\
\hline $\mathrm{C}$ & 0.32296000 & -2.57045000 & 0.53200700 \\
\hline $\mathrm{C}$ & -0.97102800 & -3.05128900 & 0.38300500 \\
\hline $\mathrm{C}$ & -1.70990800 & -2.74289500 & -0.77082200 \\
\hline C & -1.08916500 & -2.03894400 & -1.81633900 \\
\hline C & 0.20474700 & -1.55832900 & -1.66869300 \\
\hline $\mathrm{H}$ & 2.21584500 & -2.16940800 & -0.70116000 \\
\hline $\mathrm{H}$ & 0.86919000 & -2.77979800 & 1.44877800 \\
\hline $\mathrm{H}$ & -1.45299700 & -3.59427000 & 1.18958900 \\
\hline $\mathrm{C}$ & -3.19614500 & -3.00328800 & -0.80598300 \\
\hline $\mathrm{H}$ & -3.52780200 & -3.27957900 & -1.81360400 \\
\hline $\mathrm{H}$ & -3.46190300 & -3.81318700 & -0.12104000 \\
\hline $\mathrm{C}$ & -3.94736000 & -1.71701800 & -0.36484800 \\
\hline $\mathrm{H}$ & -3.79351200 & -0.92850700 & -1.10459800 \\
\hline $\mathrm{H}$ & -5.02164600 & -1.92555900 & -0.30556800 \\
\hline $\mathrm{C}$ & -3.44880500 & -1.31529400 & 1.02404900 \\
\hline O & -3.48916300 & -2.12118000 & 1.95082000 \\
\hline $\mathrm{N}$ & -2.83473700 & -0.09155100 & 1.17193600 \\
\hline C & -3.09859500 & 1.01144100 & 0.30488500 \\
\hline $\mathrm{C}$ & -2.09863000 & 1.68745800 & -0.43301900 \\
\hline $\mathrm{C}$ & -4.43206400 & 1.43507900 & 0.19600800 \\
\hline $\mathrm{C}$ & -2.48517200 & 2.76110700 & -1.25283900 \\
\hline C & -4.79475700 & 2.49569000 & -0.63002400 \\
\hline $\mathrm{H}$ & -5.18358100 & 0.91242100 & 0.78076100 \\
\hline $\mathrm{C}$ & -3.81154700 & 3.16795400 & -1.35681900 \\
\hline $\mathrm{H}$ & -1.70884100 & 3.25957800 & -1.82174500 \\
\hline $\mathrm{H}$ & -5.83598700 & 2.79958600 & -0.69441000 \\
\hline $\mathrm{H}$ & -4.07469800 & 4.00211300 & -2.00083800 \\
\hline $\mathrm{C}$ & -2.21527600 & 0.17259700 & 2.47663800 \\
\hline $\mathrm{H}$ & -1.68262300 & 1.11987300 & 2.42478100 \\
\hline $\mathrm{H}$ & -1.50508800 & -0.62351900 & 2.70455000 \\
\hline $\mathrm{H}$ & -2.97887600 & 0.20546700 & 3.26229500 \\
\hline $\mathrm{C}$ & -0.62886700 & 1.34381000 & -0.45092500 \\
\hline O & -0.18382200 & 0.59154600 & 0.48673400 \\
\hline O & 0.07504300 & 1.83013700 & -1.37101200 \\
\hline $\mathrm{H}$ & -1.65207700 & -1.82126500 & -2.72162600 \\
\hline $\mathrm{H}$ & 0.64945400 & -0.95942800 & -2.45857700 \\
\hline $\mathrm{Pd}$ & 1.77636900 & -0.02817200 & 0.29891300 \\
\hline $\mathrm{C}$ & 2.74459900 & 2.86280900 & 0.42567100 \\
\hline O & 2.55300300 & 1.83760700 & 1.09778200 \\
\hline O & 2.20425300 & 3.12024900 & -0.73642800 \\
\hline $\mathrm{C}$ & 3.66216400 & 3.94852800 & 0.92467500 \\
\hline $\mathrm{H}$ & 4.43468300 & 4.14209000 & 0.17344200 \\
\hline $\mathrm{H}$ & 4.11982400 & 3.65013300 & 1.86785600 \\
\hline
\end{tabular}


$\mathrm{H}$

$\mathrm{H}$

C

O

O

C

$\mathrm{H}$

$\mathrm{H}$

$\mathrm{H}$
3.09383500

1. 45421000

4.16145000

3.75907900

3.40886800

5.65265800

5.97201600

5.87063500

6.19351200
4.87517100

2.46807100

$-1.65526600$

$-0.58554100$

$-2.54123400$

$-1.90306100$

$-1.94234800$

$-2.87777400$

$-1.11543800$
1.05583300

$-0.99172200$

$-0.34582000$

0.19991100

$-0.86339700$

$-0.39426800$

$-1.44086400$

0.05238400

0.13128000

\section{p-TS5-cf3}

C

C

C

C

C

C

$\mathrm{H}$

$\mathrm{H}$

$\mathrm{H}$

$\mathrm{H}$

C

$\mathrm{H}$

$\mathrm{H}$

C

$\mathrm{H}$

$\mathrm{H}$

C

O

$\mathrm{N}$

C

C

C

C

C

$\mathrm{H}$

C

$\mathrm{H}$

$\mathrm{H}$

$\mathrm{H}$

C

$\mathrm{H}$

$\mathrm{H}$
$-0.98457900$

$-0.04783300$

1.17774100

1.51872700

0.56860400

$-0.65843700$

$-2.25369400$

$-0.27027400$

1.91619600

0.82054800

2.94109200

2. 97732300

3. 46282000

3.68575700

3.25190400

4.73749600

3.67071300

4.21089600

3.01594700

3.15031800

2.07519100

4. 42912500

2.32594300

4.65707000

5.24840400

3.59567800

1.49382500

5.65843700

3. 75816000

2.39594900

1.90204700

3.14702300
$-1.84353800$

$-1.68722300$

$-2.33912200$

$-3.15235500$

$-3.38203200$

$-2.73282700$

$-2.05219900$

$-1.00229500$

$-2.15700000$

$-4.03849600$

$-3.62039400$

$-4.61790100$

$-3.65854700$

$-2.61665100$

$-2.64037300$

$-2.91340300$

$-1.23019500$

$-1.08135500$

$-0.20381600$

1.11389900

1.83500300

1.68434600

3. 11431700

2. 95572500

1.10184500

3.67893800

3.64880400

3.37681700

4.67210500

$-0.33454100$

0.60548600

$-0.54754600$
$-0.38546200$

$-1.43439000$

$-1.40302800$

$-0.31016300$

0.69925700

0.66737400

$-0.81284800$

$-2.24811300$

$-2.17825400$

1.52985400

$-0.12955000$

0.32363900

$-1.08984000$

0.78937600

1.79095100

0.87008500

0.14060600

$-0.95180400$

0.78481200

0.22884400

$-0.32982300$

0.23719800

$-0.85677200$

$-0.27934400$

0.64554000

$-0.82741100$

$-1.30115500$

$-0.25679100$

$-1.23643600$

2.10968100

2. 34653300

2.88134100 


\section{$p$-TS5-cf4}

$\mathrm{C}$
$\mathrm{C}$
$\mathrm{C}$
$\mathrm{C}$
$\mathrm{C}$
$\mathrm{C}$
$\mathrm{H}$
$\mathrm{H}$
$\mathrm{H}$
$\mathrm{H}$
$\mathrm{C}$
$\mathrm{H}$
$\mathrm{H}$
$\mathrm{C}$
$\mathrm{H}$
$\mathrm{H}$
$\mathrm{C}$
$\mathrm{O}$
$\mathrm{N}$
$\mathrm{C}$

C

C

C

C

C

$\mathrm{H}$

C

C

$\mathrm{H}$

C

C

$$
\begin{array}{r}
1.62790300 \\
0.64837100 \\
0.22828300 \\
-0.06126100
\end{array}
$$

$-1.74351200$

$-2.69072200$

$-2.46179200$

$-2.19738100$

$-3.59685600$

$-4.03051700$

$-4.38721600$

$-3.02519400$

$-1.44480000$

$-4.18582100$

$-3.74384900$

$-3.46507900$

$-5.68568300$

$-6.19624800$

$-5.94650800$

$-6.00075900$

$-1.37759600$
$-1.10984200$

1.36433300

0.55863300

1.82271400

$-0.02244200$

2.88030900

1.87393000

3.11122800

3. 97117900

3. 68448400

4.15653600

4.89907500

2.46070200

$-1.48572400$

$-0.52408600$

$-2.31569800$

$-1.66112300$

$-0.95073100$

$-2.68734600$

$-1.50839600$

$-2.89964600$
2.10468100

$-0.42644300$

0.48187500

$-1.35525000$

0.32037800

0.42048300

1.10968400

$-0.76757500$

0.92845000

1.88649600

0.19407400

1.03502400

$-1.01983700$

$-0.44888200$

0.24858800

$-1.08881600$

$-0.53378800$

0.11725800

$-0.25894300$

$-1.57138700$

1.46614100
$-1.07360700$

$-0.92552800$

0.23619500

1.29838700

1.14482700

$-0.01306000$

$-2.30732000$

$-1.73673600$

0.34078000

1.97198500

2.62953100

3.21454300

2.48575600

3.44041300

4.42341400

2.92235200

3.65359600

4.06615800

3.29946200

3.33503600
$-1.67562700$

$-2.24342700$

$-2.91399200$

$-3.03506500$

$-2.52428200$

$-1.84562300$

$-1.96322700$

$-2.14947000$

$-3.32547100$

$-2.60275200$

$-3.59154600$

$-3.92405400$

$-4.44763600$

$-2.50264500$

$-2.90541100$

$-2.23098400$

$-1.31518800$

$-1.51458500$

$-0.06265900$

1.01205500
$-0.51727000$

0.77311000

1.13299700

0.22225500

$-1.07798700$

$-1.43668600$

$-0.99737500$

1. 49129400

2.13489200

$-1.77582200$

0.67288400

$-0.18822400$

1.34270800

1.42181600

1.69530300

2. 34381600

0.47992500

$-0.65906000$

0.92919000

$-0.02337500$ 


\begin{tabular}{|c|c|c|c|}
\hline $\mathrm{C}$ & 2.14859900 & 1.57230600 & -0.53072700 \\
\hline $\mathrm{C}$ & 4.56947500 & 1.45578900 & -0.50419500 \\
\hline $\mathrm{C}$ & 2.22908100 & 2.56237900 & -1.52002600 \\
\hline $\mathrm{C}$ & 4.63873300 & 2.45825500 & -1.46968900 \\
\hline $\mathrm{H}$ & 5.47143200 & 0.98563600 & -0.12687500 \\
\hline $\mathrm{C}$ & 3.46369600 & 3.01385700 & -1.98011000 \\
\hline $\mathrm{H}$ & 1.30686800 & 2.96821200 & -1.92340900 \\
\hline $\mathrm{H}$ & 5.60679700 & 2.79353300 & -1.83136900 \\
\hline $\mathrm{H}$ & 3.50829200 & 3.78830300 & -2.74072600 \\
\hline $\mathrm{C}$ & 3.02869600 & 0.24398900 & 2.34067600 \\
\hline $\mathrm{H}$ & 2.08542100 & -0.19403400 & 2.67324000 \\
\hline $\mathrm{H}$ & 3.85401500 & -0.09771500 & 2.97619100 \\
\hline $\mathrm{H}$ & 2.93381000 & 1.32437400 & 2.43790300 \\
\hline $\mathrm{C}$ & 0.78471700 & 1.17916400 & -0.03579100 \\
\hline 0 & -0.08806200 & 0.98895600 & -0.95923500 \\
\hline 0 & 0.55535700 & 1.11607500 & 1.19374700 \\
\hline $\mathrm{Pd}$ & -1.91184700 & 0.24222400 & -0.39112000 \\
\hline C & -4.24671700 & -1.50169000 & -0.52012000 \\
\hline O & -3.53430500 & -2.31703900 & -1.18632800 \\
\hline $\mathrm{C}$ & -5.72319600 & -1.80182600 & -0.39149400 \\
\hline $\mathrm{H}$ & -6.17108400 & -1.19919000 & 0.39986300 \\
\hline $\mathrm{H}$ & -6.20958700 & -1.56200000 & -1.34378500 \\
\hline $\mathrm{H}$ & -5.87023000 & -2.86777200 & -0.20083800 \\
\hline $\mathrm{C}$ & -2.41840100 & 2.84220300 & 1.09537700 \\
\hline 0 & -2.66482500 & 2.23395400 & 0.04279400 \\
\hline 0 & -1.47135500 & 2.54527500 & 1.94860300 \\
\hline $\mathrm{C}$ & -3.23575400 & 4.04215200 & 1.49791400 \\
\hline $\mathrm{H}$ & -3.62964900 & 3.89138200 & 2.50800500 \\
\hline $\mathrm{H}$ & -2.59115800 & 4.92695500 & 1.52671100 \\
\hline $\mathrm{H}$ & -4.05203600 & 4.19513400 & 0.79202500 \\
\hline $\mathrm{H}$ & -0.80260400 & 1.84522100 & 1.61978400 \\
\hline O & -3.81348100 & -0.44454700 & 0.03483200 \\
\hline $\mathrm{H}$ & -0.09050900 & -1.40485900 & -2.42588200 \\
\hline
\end{tabular}

\section{$o_{1}$-TS5-cf1}

$\mathrm{C}$
$\mathrm{C}$
$\mathrm{C}$
$\mathrm{C}$
$\mathrm{C}$
$\mathrm{C}$
$\mathrm{H}$
$\mathrm{C}$

$$
\begin{array}{r}
0.23887900 \\
1.14908900 \\
0.93435100 \\
-0.22831500 \\
-1.11841300 \\
-0.90358000 \\
2.05227500 \\
-0.48777700
\end{array}
$$$$
-2.69563800
$$$$
-2.54105700
$$$$
-1.98184800
$$$$
-1.77175400
$$$$
-1.75580600
$$$$
-0.38804600
$$$$
-2.29399000
$$$$
0.22687200
$$$$
-3.03649400
$$$$
-0.55783400
$$$$
-3.21682200
$$$$
-1.92463300
$$$$
-2.00045000
$$$$
0.31012400
$$$$
-2.15454200
$$

1. 71296600 
$\mathrm{H}$

$\mathrm{H}$

C

$\mathrm{H}$

$\mathrm{H}$

C

O

$\mathrm{N}$

C

C

C

C

C

$\mathrm{H}$

C

$\mathrm{H}$

$\mathrm{H}$

$\mathrm{H}$

C

$\mathrm{H}$

$\mathrm{H}$

$\mathrm{H}$

C

0

O

$\mathrm{H}$

$\mathrm{H}$

$\mathrm{H}$

$\mathrm{H}$

$\mathrm{Pd}$

C

O

O

C

$\mathrm{H}$

$\mathrm{H}$

$\mathrm{H}$

$\mathrm{H}$

C

O

O

C

$\mathrm{H}$

$\mathrm{H}$
$-0.44818100$

$-1.85343600$

$-2.61012900$

$-2.20079700$

$-1.74338700$

$-0.94662500$

$-2.64256400$

$-3.29974000$

$-2.64657600$

$-4.66446300$

$-3.38314600$

$-5.38160100$

$-5.14995400$

$-4.73791400$

$-2.86345300$

$-6.43921100$

$-5.28930500$

$-2.46860100$

$-1.63591400$

$-2.24198500$

$-3.39310100$

$-1.17637900$

$-0.47054900$

$-0.72338300$

$-1.99964300$

$-1.62614100$

0.41006500

2.05208600

1. 51426500

1.84214400

2.03502300

1.10431700

2.48782500

3.05789500

3.14074700

1.70923900

0.53008600

3.96641300

3.54061700

3.23726400

5.44964500

5.61994600

6.00392000
$-3.16364700$

2.14440700

$-1.57542400$

2.19292900

$-1.52244000$

2.08426400

$-1.74013500$

1.32763900

$-1.96902000$

3.02341600

$-0.03002100$

2.38536800

0.37233200

3. 22236400

0.82295600

1. 76778300

0.49307300

0.53479000

0.54070300

$-0.71727100$

0.19272500

0.58357700

0.28508800

$-1.88174900$

$-0.08037900$

$-0.58150800$

0.17595600

1.55477100

$-0.03264700$

0.33689000

$-1.81969500$

$-2.83247400$

$-0.32239200$

$-0.23819200$

$-0.52127500$

$-2.73289600$

2.25434600

2.02801400

2. 66027600

1.43929000

2.39321100

3.08547600

2.77696200

1.76978200

0.81365700

$-0.87587700$

0.61518100

0.18107500

1.18108800

$-1.98026800$

$-3.47600600$

$-3.78003600$

$-2.84796800$

$-1.58937100$

0.23138600

3.17019500

2. 32977300

3. 01067500

4.52950100

4.71860700

4.58218500

5.29672700

2.16414100

$-1.27348700$

$-0.16045500$

$-0.09640100$

$-2.51007300$

$-3.60283000$

$-2.23311200$

0.04733100

$-0.71890600$

0.17315800

$-1.78648900$

$-0.63459500$

$-1.54971300$

0.23655400

$-0.56923300$

$-1.81310900$

0.52685100

0.08445600

$-2.28347700$

0.77471100

$-1.38684200$

0.79897600

$-1.18022500$

1. 86194400

$-0.65554700$

0.20837600 


\section{$o_{1}$-TS5-cf2}

C

C

C

C

C

$\mathrm{H}$

$\mathrm{H}$

$\mathrm{H}$

$\mathrm{H}$

C

$\mathrm{H}$

$\mathrm{H}$

C

$\mathrm{H}$

$\mathrm{H}$

C

$\mathrm{O}$

$\mathrm{N}$

C

C

C

C

C

$\mathrm{H}$

C

$\mathrm{H}$

$\mathrm{H}$

$\mathrm{H}$

C

$\mathrm{H}$

$\mathrm{H}$

$\mathrm{H}$

C

O

O

$\mathrm{H}$

$\mathrm{Pd}$

C

O
$-1.62668900$

$-2.09882700$

$-1.43750700$

$-0.27329300$

0.16743800

$-0.48646400$

$-2.13773700$

$-2.29685100$

1.04045800

$-0.10969100$

0.44088400

0.09718600

0.16379900

1.98618800

2.38749000

2.27807900

2. 57684900

2.29344600

3. 43224700

3.69103600

2.68610600

5.00264500

3.00745800

5.32196300

5.76478800

4.31981900

2.21091300

6.34673000

4.55780400

3.84923200

3.76053100

3.19608100

4.88475000

1.26334300

0.39249600

1.02828400

$-3.00635200$

$-1.56763100$

$-1.62818500$

$-1.80915400$
3.78796000

2.55419300

1.82244900

2.37585300

3.63496200

4. 32352100

4.33457500

1.45811600

4.08308300

5.29311500

1.64600300

0.61012600

2.08213800

1.67330400

2.58329500

1.69079100

0.49470600

0.33716700

$-0.36285700$

$-0.32348000$

$-0.52962200$

$-0.07820900$

$-0.44218900$

$-0.03910100$

0.09504500

$-0.21766300$

$-0.55969800$

0.14937300

$-0.17252400$

$-1.58777900$

$-1.42509600$

$-2.41540200$

$-1.82544700$

$-0.80015300$

$-0.17888300$

$-1.55410000$

2.14293700

$-0.25343200$

$-3.29689600$

$-2.32581000$
$-1.61918600$

$-1.19076100$

$-0.17080900$

0.43441100

0.01324600

$-1.00975000$

$-2.40665000$

0.74663300

0.48023900

$-1.32621800$

1.54824300

1. 58306000

2. 51579700

1. 41690400

1.87719000

0.36654500

2. 18423700

3. 36626300

1.51716400

0.11116900

$-0.85427400$

$-0.31122500$

$-2.21473400$

$-1.66769400$

0.44307500

$-2.62445700$

$-2.94217800$

$-1.97567600$

$-3.68335200$

2.20598500

3.27946400

1.90625700

1.94604600

$-0.45397500$

$-1.16533900$

0.51622200

$-1.62632100$

$-0.58614600$

$-0.42636700$

$-1.17922800$ 
$\mathrm{O}$
$\mathrm{H}$
$\mathrm{C}$
$\mathrm{O}$
$\mathrm{O}$
$\mathrm{C}$
$\mathrm{H}$
$\mathrm{H}$
$\mathrm{H}$
$\mathrm{C}$
$\mathrm{H}$
$\mathrm{H}$
$\mathrm{H}$

$\mathrm{H}$

\section{$o_{1}$-TS5-cf3}

C

C

C

C

C

C

$\mathrm{H}$

$\mathrm{H}$

$\mathrm{H}$

$\mathrm{H}$

C

$\mathrm{H}$

$\mathrm{H}$

C

$\mathrm{H}$

$\mathrm{H}$

C

O

$\mathrm{N}$

C

C

C

C

C

$\mathrm{H}$

C

$\mathrm{H}$

$\mathrm{H}$
$-0.84237400$

$-0.21937400$

$-3.99136300$

$-3.55249200$

$-3.35879200$

$-2.32708000$

$-1.58413000$

$-2.83975600$

$-3.03953800$

$-5.36330600$

$-5.23647400$

$-5.89578800$

$-5.93040300$
$-3.30048800$

$-2.48640000$

0.28130700

$-0.38777100$

1.20966400

$-4.60668700$

$-5.37417200$

$-4.92434400$

$-4.50284100$

$-0.08719200$

$-0.73050900$

0.81261000

$-0.63086800$
0.61666000

0.63011100

0.95002000

$-0.04234400$

1.53265200

$-0.67713700$

$-0.91879100$

0.23631500

$-1.49550200$

1.47189900

2.35028500

1.78804100

0.71463900
$-1.44358100$

$-0.61907300$

$-0.22342900$

$-0.59225900$

$-1.47533200$

$-1.88586000$

$-1.75270300$

$-0.29298800$

0.39250900

$-2.48805600$

$-0.17031700$

$-0.46209700$

$-0.73761400$

1.33219000

1.42436600

1.70534100

2.19182300

2.25978700

2. 90398100

3.55120300

2.88473200

4.90953200

3.61492400

5.61807600

5.39797300

4.96873700

3.09250100

6.67287500
$-2.75795900$

$-3.73968900$

$-3.66243200$

$-2.58047000$

$-1.60436700$

$-1.71721900$

$-2.82609700$

$-4.58193600$

$-4.45688200$

$-1.47762700$

$-2.52764600$

$-1.56981900$

$-3.30433900$

$-2.76447700$

$-2.98619200$

$-3.63754000$

$-1.51445500$

$-0.67994200$

$-1.37110400$

$-0.10683500$

0.97384800

$-0.00496400$

2.13110200

1.16149800

$-0.85560200$

2. 23123500

2. 94978000

1.22999600
2.64907200

2.08848500

0.75215200

$-0.05330300$

0.48948100

1.84293800

3.68805100

2. 69379900

0.33885200

$-0.28577500$

$-1.50854100$

$-1.94860200$

$-2.04122700$

$-1.80651700$

$-2.87319800$

$-1.26336900$

$-1.60372100$

$-2.49503900$

$-0.42794900$

$-0.20351800$

0.40594600

$-0.50520400$

0.71260500

$-0.21754700$

$-0.97041400$

0.40096900

1.19539800

$-0.46935900$ 
$\mathrm{H}$

C

$\mathrm{H}$

$\mathrm{H}$

$\mathrm{H}$

C

O

$\mathrm{O}$

$\mathrm{Pd}$

C

O

C

$\mathrm{H}$

$\mathrm{H}$

$\mathrm{H}$

C

O

O

C

$\mathrm{H}$

$\mathrm{H}$

$\mathrm{H}$

$\mathrm{H}$

O

$\mathrm{H}$
5.51337600

2.71639700

1.87574400

2.53804300

3. 63197200

1.40799100

0.68527000

0.98604800

$-1.32775000$

$-4.10271200$

$-3.72154300$

$-5.52615000$

$-5.52732400$

$-6.16400900$

$-5.90346900$

$-1.32857800$

$-1.26468700$

$-0.88837500$

$-1.93271700$

$-2.37027100$

$-2.69539700$

$-1.15787400$

$-0.26640600$

$-3.36498100$

$-2.58361000$
3.14090600

0.63795200

$-2.16475200$

0.78390300

$-1.79828600$

1.38496500

$-3.21187800$

0.54015300

$-2.10826500$

1.37928700

1.00272400

0.70066800

0.21450700

$-0.01339700$

1.80111200

1.56636800

0.44005900

$-0.21312600$

$-1.39973200$

0.16897700

0.62839300

$-0.71615600$

0.90594800

3.49283600

2. 52216700

3. 49485800

4.79680800

4.68828000

5.10793400

5.57067600

2.69243000

0.71601400

$-0.98441900$
$-0.07353200$

$-0.70588100$

$-0.91109400$

$-1.05305600$

$-2.04821000$

$-1.07282500$

$-0.34047500$

0.07996600

$-0.69251200$

1.30944800

$-0.36761800$

$-1.35997500$

0.35335800

$-0.37979100$

1.47052300

$-0.23611700$

2. 24004500

\section{$o_{1}$-TS5-cf4}

C

C

C

C

C

C

$\mathrm{H}$

$\mathrm{H}$

$\mathrm{H}$

$\mathrm{H}$

C

$\mathrm{H}$

$\mathrm{H}$

C

$\mathrm{H}$

$\mathrm{H}$
0.73744800

$-0.15034100$

$-0.30325700$

0.39061300

1.29173300

1. 45035500

0.86609900

$-0.72326300$

$-0.99979100$

2.47945900

0.24048700

1.23974800

$-0.18194900$

$-0.62263700$

$-0.08981500$

$-0.76654600$
$-2.39463400$

$-3.31691700$

$-3.40615300$

$-2.55387000$

$-1.60376400$

$-1.55222300$

$-2.34057600$

$-3.98050400$

$-4.12371700$

$-1.71180000$

$-2.75276500$

$-2.74125000$

$-3.74765900$

$-1.72363500$

$-0.77321300$

$-2.08535700$
2.89719200

2. 33203400

0.94882900

0.08295900

0.64494900

2. 05584600

3. 97456000

2. 97491900

0.52969100

0.04691200

$-1.41591500$

$-1.87064400$

$-1.58391900$

$-2.18069100$

$-2.25256300$

$-3.20829900$ 


\begin{tabular}{|c|c|c|c|}
\hline C & -1.97751400 & -1.51617500 & -1.50941700 \\
\hline O & -2.45775500 & -2.36106900 & -0.76532600 \\
\hline $\mathrm{N}$ & -2.63668900 & -0.31716400 & -1.74176600 \\
\hline $\mathrm{C}$ & -3.44858500 & 0.16710900 & -0.65691500 \\
\hline $\mathrm{C}$ & -2.88028200 & 0.77242900 & 0.48958200 \\
\hline C & -4.83499200 & 0.06988500 & -0.77297200 \\
\hline $\mathrm{C}$ & -3.73723900 & 1.26738900 & 1.48277100 \\
\hline C & -5.67279800 & 0.55771700 & 0.23024500 \\
\hline $\mathrm{H}$ & -5.24189200 & -0.40467800 & -1.66029100 \\
\hline $\mathrm{C}$ & -5.12064400 & 1.15914900 & 1.36219400 \\
\hline $\mathrm{H}$ & -3.28926700 & 1.73968200 & 2.35013600 \\
\hline $\mathrm{H}$ & -6.75070000 & 0.46623400 & 0.12716200 \\
\hline $\mathrm{H}$ & -5.76428700 & 1.54375300 & 2.14843200 \\
\hline $\mathrm{C}$ & -2.12971800 & 0.69938500 & -2.66114500 \\
\hline $\mathrm{H}$ & -2.95262400 & 1.37322500 & -2.91549700 \\
\hline $\mathrm{H}$ & -1.78605400 & 0.22915100 & -3.58599800 \\
\hline $\mathrm{H}$ & -1.30796500 & 1.28287500 & -2.22658400 \\
\hline C & -1.39714900 & 0.94360700 & 0.71498200 \\
\hline O & -0.63762700 & 0.31341500 & -0.10621600 \\
\hline O & -0.99799400 & 1.67509700 & 1.64791400 \\
\hline $\mathrm{Pd}$ & 1.38871900 & 0.36647800 & -0.07712400 \\
\hline $\mathrm{C}$ & 1.41029700 & 3.41807300 & 0.10968900 \\
\hline O & 1.45619100 & 2.46158200 & -0.68219700 \\
\hline O & 0.85116500 & 3.39843500 & 1.28868500 \\
\hline C & 2.02953500 & 4.74338400 & -0.25158000 \\
\hline $\mathrm{H}$ & 2.75842100 & 5.02050000 & 0.51708600 \\
\hline $\mathrm{H}$ & 1.25626400 & 5.51835200 & -0.26263000 \\
\hline $\mathrm{H}$ & 2.51627400 & 4.67949200 & -1.22482200 \\
\hline $\mathrm{H}$ & 0.24271700 & 2.58099000 & 1.44123700 \\
\hline $\mathrm{C}$ & 4.13980100 & -0.60090700 & -0.43892400 \\
\hline O & 3.44755100 & 0.44097400 & -0.22487900 \\
\hline O & 3.69023600 & -1.78956700 & -0.41935200 \\
\hline $\mathrm{C}$ & 5.61352500 & -0.41796400 & -0.72675900 \\
\hline $\mathrm{H}$ & 6.19447400 & -0.91641400 & 0.05593600 \\
\hline $\mathrm{H}$ & 5.87278900 & 0.64037900 & -0.76944600 \\
\hline $\mathrm{H}$ & 5.85613900 & -0.90570100 & -1.67584700 \\
\hline $\mathrm{H}$ & 2.16154300 & -0.84727000 & 2.47980100 \\
\hline
\end{tabular}

\section{$o_{1}$-TS5-cf5}

$\mathrm{C}$
$\mathrm{C}$
$\mathrm{C}$
$\mathrm{C}$

$-2.88028200$

$-4.83499200$

$-5.67279800$

$-5.67279800$

$-5.24189200$

5.12064400

$-3.28926700$

$-6.75070000$

700

$-2.12971800$

$-1.30796500$

$-0.63762700$

1.38871900

1.41029700

6500

2.02953500

.25626400

0.24271700

4.13980100

3.44755100

3.69023600

5.61352500

5.194278900

5.85613900

$-0.84727000$
$-1.50941700$

$-0.76532600$

$-0.65691500$

.

0.77297200

0.23024500

$-1.66029100$

1.36219400

2.35013600

2.14843200

$-2.66114500$

$-3.58599800$

$-2.22658400$

0.71498200

.10621600

1.64791400

0.07712400

$-0.68219700$

1.28868500

$-0.25158000$

0.51708600

$-0.26263000$

$-1.22482200$

1.44123700

$-0.43892400$

$-0.22487900$

5200

0.72675900

5593600

$-1.67584700$

2.47980100
$-1.52856100$

$\begin{array}{rr}3.35427800 & -2.13857400 \\ 2.16587900 & -1.52859800 \\ 1.76044500 & -0.28157400 \\ 2.60802200 & 0.36573900\end{array}$

$-1.90714300$

$-1.36042200$

$-0.42273700$ 


\begin{tabular}{|c|c|c|c|}
\hline $\mathrm{C}$ & -0.10538400 & 3.83314600 & -0.23452500 \\
\hline $\mathrm{C}$ & -0.62445500 & 4.19235300 & -1.47764700 \\
\hline $\mathrm{H}$ & -2.31212000 & 1.43804800 & 0.56029000 \\
\hline $\mathrm{H}$ & 0.56204700 & 4.52315800 & 0.27517800 \\
\hline $\mathrm{C}$ & 0.11250500 & 2.28408200 & 1.74463900 \\
\hline $\mathrm{H}$ & -0.41146100 & 2.93570400 & 2.45812200 \\
\hline $\mathrm{H}$ & -0.14076700 & 1.25529000 & 2.01694200 \\
\hline $\mathrm{C}$ & 1.63496000 & 2.48813400 & 1.94783400 \\
\hline $\mathrm{H}$ & 1.98050800 & 3.40945700 & 1.47244100 \\
\hline $\mathrm{H}$ & 1.82292200 & 2.58534600 & 3.02018000 \\
\hline $\mathrm{C}$ & 2.42343800 & 1.24892000 & 1.53695800 \\
\hline 0 & 2.48638800 & 0.30956200 & 2.32212600 \\
\hline $\mathrm{N}$ & 3.04681900 & 1.20609300 & 0.30504600 \\
\hline $\mathrm{C}$ & 3.64386600 & -0.04358800 & -0.07617600 \\
\hline $\mathrm{C}$ & 2.88826400 & -1.22376200 & -0.15895100 \\
\hline $\mathrm{C}$ & 5.01063000 & -0.07295600 & -0.35614000 \\
\hline $\mathrm{C}$ & 3.52665800 & -2.42153500 & -0.49556300 \\
\hline $\mathrm{C}$ & 5.63125000 & -1.26430400 & -0.73405600 \\
\hline $\mathrm{H}$ & 5.58319300 & 0.84519100 & -0.25874400 \\
\hline $\mathrm{C}$ & 4.88744500 & -2.44363100 & -0.79912800 \\
\hline $\mathrm{H}$ & 2.93762500 & -3.33286400 & -0.51377400 \\
\hline $\mathrm{H}$ & 6.69482500 & -1.27290600 & -0.95606800 \\
\hline $\mathrm{H}$ & 5.36631900 & -3.37923000 & -1.07400100 \\
\hline $\mathrm{C}$ & 2.77047200 & 2.12079300 & -0.80367500 \\
\hline $\mathrm{H}$ & 3.63206800 & 2.12536100 & -1.47619000 \\
\hline $\mathrm{H}$ & 2.61759100 & 3.13753400 & -0.44279800 \\
\hline $\mathrm{H}$ & 1.88253900 & 1.79812400 & -1.35794000 \\
\hline $\mathrm{C}$ & 1.41094800 & -1.26626300 & 0.12070300 \\
\hline 0 & 0.73009000 & -0.31086900 & -0.42346800 \\
\hline 0 & 0.97245900 & -2.21503200 & 0.79510600 \\
\hline $\mathrm{Pd}$ & -1.30500500 & -0.36905600 & -0.34790400 \\
\hline $\mathrm{C}$ & -1.78338100 & -3.33940500 & -0.14387800 \\
\hline 0 & -1.53071900 & -2.44080000 & -0.96793300 \\
\hline 0 & -1.22850500 & -3.42381000 & 1.03185000 \\
\hline $\mathrm{C}$ & -2.80662200 & -4.39435100 & -0.46411400 \\
\hline $\mathrm{H}$ & -2.67777500 & -5.27759600 & 0.16347600 \\
\hline $\mathrm{H}$ & -2.74527300 & -4.65330400 & -1.52350700 \\
\hline $\mathrm{H}$ & -3.79854000 & -3.96300500 & -0.28382600 \\
\hline $\mathrm{H}$ & -0.40801100 & -2.79543100 & 1.04400300 \\
\hline $\mathrm{C}$ & -3.99555800 & 0.24403700 & 0.59768300 \\
\hline 0 & -3.37401600 & -0.49570900 & -0.23516700 \\
\hline 0 & -3.48802800 & 1.23830200 & 1.19358100 \\
\hline $\mathrm{C}$ & -5.43254200 & -0.12485600 & 0.89543400 \\
\hline $\mathrm{H}$ & -5.98309700 & 0.74697500 & 1.25266900 \\
\hline
\end{tabular}


$\mathrm{H}$

$\mathrm{H}$

$\mathrm{H}$

$\mathrm{H}$

$\mathrm{H}$
$-5.43688300$

$-5.90571200$

$-0.33793600$

$-1.94499400$

$-2.65769700$
$-0.88853800$

$-0.54828100$

5.14147200

3.64086500

1.53678200
1.68195500

0.00678500

$-1.92378300$

$-3.10002100$

$-2.00020400$

\section{$o_{1}$-TS5-cf6}

C

C

C

C

C

C

$\mathrm{H}$

$\mathrm{H}$

$\mathrm{H}$

C

$\mathrm{H}$

$\mathrm{H}$

C

$\mathrm{H}$

$\mathrm{H}$

C

O

$\mathrm{N}$

C

C

C

C

C

$\mathrm{H}$

C

$\mathrm{H}$

$\mathrm{H}$

$\mathrm{H}$

C

$\mathrm{H}$

$\mathrm{H}$

$\mathrm{H}$

C

O

$O$

$\mathrm{Pd}$
$-1.01336400$

$-1.66646300$

$-1.23072800$

$-0.11265800$

0.51766700

0.08799800

$-2.54106900$

$-2.23537000$

1. 38602600

0.26245000

$-0.47799700$

0.13445400

1. 67441900

1.83465400

1.74235500

2.73133500

3.14859200

3.09068400

3.58782500

2.66410300

4.95311900

3.11919900

5.40544700

5.64882000

4.48863300

2.39679900

6.47116200

4.83862800

2.83975300

1.77462700

3.26652400

3.33439200

1.19744000

0.43803900

0.82384500

$-1.54111100$
$-3.39929500$

$-2.31717900$

$-1.75794300$

$-2.33642400$

$-3.43828700$

$-3.94638400$

$-1.89460400$

$-1.68773500$

$-3.88025900$

$-1.82740400$

$-2.23302900$

$-0.74094100$

$-2.18027900$

$-3.26129200$

$-1.83175800$

$-1.55866100$

$-2.15945200$

$-0.24789000$

0.50405000

1.09950300

0.59012400

1. 75094700

1.27548300

0.09755600

1.85017700

2.18110200

1. 34124400

2.36867100

0.44031000

0.61939500

$-0.12509100$

1.41172800

1.06179700

0.52193200

1. 55025300

0.32506000
$-2.26233400$

$-1.68767000$

$-0.45918900$

0.21352000

$-0.37015400$

$-1.59846700$

$-2.17678300$

0.39701400

0.10024400

1. 59771600

2.30323200

1. 62833000

2.12973700

2.13816200

3.16385600

1. 22316200

0.23989600

1.47773100

0.35923900

$-0.51617800$

0.09641400

$-1.66480300$

$-1.03319800$

0.76863000

$-1.91592400$

$-2.35216000$

$-1.23402600$

$-2.80422100$

2.74692300

2. 91637300

3.58263600

2.70344900

$-0.18563400$

$-1.06237000$

0.90935200

$-0.54417200$ 


$\begin{array}{lrrr}\mathrm{C} & -3.99339400 & -0.67694900 & 0.72527800 \\ \mathrm{O} & -3.33058200 & -1.68172700 & 1.12814800 \\ \mathrm{C} & -5.42523500 & -0.54587400 & 1.19675400 \\ \mathrm{H} & -6.00873400 & -1.38057500 & 0.79436200 \\ \mathrm{H} & -5.45380200 & -0.62140700 & 2.28779800 \\ \mathrm{H} & -5.85723800 & 0.40045300 & 0.86942500 \\ \mathrm{C} & -1.88016500 & 3.29782700 & 0.07468900 \\ \mathrm{O} & -1.96012800 & 2.44203500 & -0.81967800 \\ \mathrm{O} & -1.13125800 & 3.19811300 & 1.14231700 \\ \mathrm{C} & -2.66767800 & 4.57864300 & -0.00607400 \\ \mathrm{H} & -3.33607900 & 4.55553100 & -0.86676900 \\ \mathrm{H} & -3.24075900 & 4.71071600 & 0.91718600 \\ \mathrm{H} & -1.97778100 & 5.42532500 & -0.08670400 \\ \mathrm{H} & -0.46396900 & 2.42513000 & 1.06764300 \\ \mathrm{O} & -3.54935900 & 0.21865400 & -0.05873300 \\ \mathrm{H} & 0.61937200 & -4.78804200 & -2.03556000 \\ \mathrm{H} & -1.35012800 & -3.81322000 & -3.20864400\end{array}$

\section{$o_{1}$-TS5-cf7}

C

C

C

C

C

C

$\mathrm{H}$

$\mathrm{H}$

$\mathrm{H}$

$\mathrm{H}$

$\mathrm{H}$

C

$\mathrm{H}$

$\mathrm{H}$

C

$\mathrm{H}$

$\mathrm{H}$

C

O

$\mathrm{N}$

C

C

C

C
0.95909200

$-0.13344700$

$-0.56893100$

0.02950600

1.16384800

1.60842400

1. 30469600

$-0.63975500$

$-1.39940900$

2.17155000

2. 49556100

$-0.45952900$

0.33246500

$-0.63914600$

$-1.76800600$

$-1.54938500$

$-2.14682500$

$-2.81186400$

$-3.21580800$

$-3.16553000$

$-3.42732600$

$-2.56852000$

$-4.66912500$

$-2.99568400$
$-2.87581900$

$-3.58521500$

$-3.38830300$

$-2.42985300$

$-1.72577300$

$-1.97042200$

$-3.04799100$

$-4.31960600$

$-3.97105900$

$-1.86057000$

$-1.45304900$

$-2.27593200$

$-1.82041600$

$-3.28216800$

$-1.45035600$

$-0.38693700$

$-1.70926400$

$-1.84981600$

$-3.00741800$

$-0.91074100$

0.47083900

1. 51800200

0.77771500

2.84355300
2.47379400

1.96306400

0.65212800

$-0.17830700$

0.31983100

1. 64713500

3.48920000

2.58494900

0.27050300

$-0.50806600$

2.00405600

$-1.60959500$

$-2.21409200$

$-2.00361600$

$-1.84352100$

$-1.80645900$

$-2.83797600$

$-0.81657500$

$-0.74290900$

0.14172500

$-0.17638200$

0.20306400

$-0.74672100$

0.04801800 
C

$\mathrm{H}$

C

$\mathrm{H}$

$\mathrm{H}$

$\mathrm{H}$

C

$\mathrm{H}$

$\mathrm{H}$

$\mathrm{H}$

C

O

$\mathrm{O}$

$\mathrm{Pd}$

C

O

O

C

$\mathrm{H}$

$\mathrm{H}$

$\mathrm{H}$

$\mathrm{H}$

C

O

O

C

$\mathrm{H}$

$\mathrm{H}$

$\mathrm{H}$
$-5.06806400$

$-5.32738600$

$-4.23328600$

$-2.32943200$

$-6.03168100$

$-4.54109200$

$-3.94665900$

$-3.92015700$

$-3.49343900$

$-4.99041900$

$-1.14030000$

$-0.49763100$

$-0.68036400$

1.50916900

2.10645200

1. 94189300

1.53632800

3.01741800

2. 44106400

3.49617500

3.77444500

0.75019700

4.02020400

3.55095600

3. 34453500

5.48859400

6.04573900

5.58992300

5.88722100
2.10070500

$-0.04102600$

3.13938900

3. 63691800

2. 31608600

4.17396100

$-1.41745100$

$-0.66896900$

$-2.34599500$

$-1.62228300$

1. 32769300

0.52280100

2.00459100

0.29485900

3.26165800

2. 39728900

3.25751800

4.43754600

5.36637400

4.35294800

4.46970800

2.59521200

$-0.99266200$

0.05671300

$-2.02855600$

$-0.97070000$

$-0.35587000$

$-0.52055300$

$-1.98607000$
$-0.93500300$

$-1.02463500$

$-0.51994300$

0.37175000

$-1.38825300$

$-0.64224600$

1.27691200

2.07218400

1.62736700

1.00562300

0.63842000

$-0.12414900$

1.57901100

$-0.14075000$

0.35634800

$-0.52035900$

1. 53106400

0.11903000

0.18109600

$-0.85652100$

0.90949600

1.57766300

$-0.86899700$

$-0.31989300$

$-1.14275300$

$-1.23408800$

$-0.52405500$

$-2.22844500$

$-1.26883700$

\section{$o_{1}$-TS5-cf8}

C

C

C

C

C

C

$\mathrm{H}$

$\mathrm{H}$

$\mathrm{H}$

$\mathrm{H}$

C

$\mathrm{H}$
$-1.07003900$

$-1.64863900$

$-1.14453800$

$-0.03498600$

0.49132100

0.00738300

$-1.46134300$

$-2.12371200$

1.30478300

0.45916200

0.47892100

0.59762100
$-3.58782900$

$-2.44314100$

$-1.82901200$

$-2.41686900$

$-3.61086500$

$-4.16794600$

$-4.03942200$

$-1.64795300$

$-4.09580000$

$-5.08104700$

$-1.89933500$

$-2.76935700$
$-1.85376900$

$-1.32535900$

$-0.14613100$

0.52250600

0.00731200

$-1.17619000$

$-2.76113600$

0.71583100

0.53336100

$-1.55614800$

1.85615300

2.51029700 


$$
\begin{array}{r}
-0.28295300 \\
1.83432000 \\
2.28298700 \\
1.65093800 \\
2.80410000 \\
3.15723100 \\
3.18364000 \\
3.51282000 \\
2.56875400 \\
4.87709700 \\
3.01576100 \\
5.30308600 \\
5.61019200 \\
4.36577900 \\
2.27025400 \\
6.36523400 \\
4.68451900 \\
3.83675600 \\
3.20240200 \\
3.93855500 \\
4.81945700 \\
-1.08158400 \\
0.5
\end{array}
$$

$-1.25517300$

2.30779700

$-1.11197600$

1.88471200

$-1.26808100$

2.87130100

$-0.04712800$

1. 78581600

$-1.65892900$

0.85318200

$-2.83384200$

0.90340600

$-0.86651700$

$-0.22312900$

0.52741200

$-0.11308000$

1.56188500

0.02312200

0.86276300

$-0.15192000$

2.88810500

0.13495800

2.18594100

0.06616600

$-0.08453700$

$-0.22765200$

3.21044400

0.05983600

3. 66198400

0.28260900

2. 41160500

$-0.12500100$

4.24656100

0.12676300

$-1.60030300$

$-1.31520900$

$-2.44568100$

$-1.58874300$

$-0.92656500$

$-2.16758900$

$-1.99542500$

$-1.03153800$

1.36158800

0.12819400

0.48363700

$-0.64241800$

2.06638300

0.97292900

0.23590200

$-0.40306500$

$-0.71189800$

0.77280700

$-1.57838600$

1.39267500

$-0.56847400$

1.13666100

2.09634000

0.04371800

0.37639000

$-1.55691000$

1.26446700

3.20820600

$-0.36046500$

2.24566900

$-1.10413700$

3. 37357100

0.82686300

0.06135400

$-0.13210200$

4.30200600

$-0.78318600$

4.07234100

$-1.75874200$

4.40013100

$-0.03588500$

5.25612000

$-0.81800600$

2.72524300

0.95057200

$-2.01566400$

$-1.80353200$

\section{m-TS9}




\begin{tabular}{|c|c|c|c|}
\hline C & -0.30236600 & 1.36631700 & -2.24118200 \\
\hline C & -0.32779500 & 1.80820600 & -0.89681500 \\
\hline $\mathrm{C}$ & 0.84034700 & 2.39126200 & -0.3587430 \\
\hline C & 2.01020400 & 2.52105400 & -1.1122560 \\
\hline C & 1.99883600 & 2.05550800 & -2.43614300 \\
\hline C & 0.85198100 & 1.49408700 & -3.0060870 \\
\hline $\mathrm{H}$ & -1.19944700 & 0.93589900 & -2.6794060 \\
\hline $\mathrm{H}$ & -1.40881800 & 2.54197700 & -0.6574410 \\
\hline $\mathrm{H}$ & 0.84231300 & 2.73105200 & 0.6735110 \\
\hline $\mathrm{H}$ & 2.90539500 & 2.13944400 & -3.0325100 \\
\hline $\mathrm{H}$ & 0.87044800 & 1.15274600 & -4.0375220 \\
\hline C & 3.27999500 & 3.05474800 & -0.4858500 \\
\hline $\mathrm{H}$ & 3.05005100 & 3.85456200 & 0.2245600 \\
\hline $\mathrm{H}$ & 3.93775000 & 3.46961600 & -1.2583820 \\
\hline C & 4.03725300 & 1.94273300 & 0.27369200 \\
\hline $\mathrm{H}$ & 4.99647600 & 2.33736800 & 0.6309150 \\
\hline $\mathrm{H}$ & 4.24901900 & 1.10933700 & -0.3994540 \\
\hline C & 3.23918300 & 1.52103900 & 1.50898100 \\
\hline O & 2.84934200 & 2.37061400 & 2.3084760 \\
\hline $\mathrm{N}$ & 2.93186400 & 0.19651800 & 1.6871530 \\
\hline C & 3.39379300 & -0.85173000 & 0.8342730 \\
\hline $\mathrm{C}$ & 2.55956000 & -1.51812300 & -0.093467 \\
\hline $\mathrm{C}$ & 4.73990800 & -1.22559800 & 0.9377120 \\
\hline C & 3.11540800 & -2.53558200 & -0.885776 \\
\hline C & 5.27539300 & -2.23248000 & 0.1373350 \\
\hline $\mathrm{H}$ & 5.36027600 & -0.70345400 & 1.6600400 \\
\hline C & 4.45605400 & -2.89363000 & -0.778961 \\
\hline $\mathrm{H}$ & 2.46595600 & -3.03170200 & -1.597554 \\
\hline $\mathrm{H}$ & 6.32332800 & -2.50208900 & 0.235690 \\
\hline $\mathrm{H}$ & 4.85779900 & -3.68419200 & -1.406160 \\
\hline C & 2.12806200 & -0.14746900 & 2.866532 \\
\hline $\mathrm{H}$ & 2.71657800 & 0.00137300 & 3.7786940 \\
\hline $\mathrm{H}$ & 1.24170600 & 0.48658400 & 2.9078250 \\
\hline $\mathrm{H}$ & 1.81900000 & -1.18893200 & 2.7875220 \\
\hline $\mathrm{C}$ & 1.09867800 & -1.20907700 & -0.303890 \\
\hline O & 0.53950100 & -0.45995000 & 0.5614610 \\
\hline O & 0.53945800 & -1.74076600 & -1.307079 \\
\hline $\mathrm{Pd}$ & -1.31259700 & 0.45533700 & 0.414788 \\
\hline C & -3.21099000 & -1.70062500 & 1.3378190 \\
\hline O & -2.26284400 & -0.93919100 & 1.715228 \\
\hline C & -4.23015100 & -2.06249400 & 2.404016 \\
\hline $\mathrm{H}$ & -4.76934600 & -2.97218400 & 2.133343 \\
\hline $\mathrm{H}$ & -3.74013300 & -2.17514900 & 3.37409 \\
\hline $\mathrm{H}$ & -4.94513700 & -1.23519700 & 2.48424 \\
\hline
\end{tabular}


C

O

C

$\mathrm{H}$

$\mathrm{H}$

$\mathrm{H}$

O

O

Ag
$-3.36005900$

$-3.15846900$

$-4.74585200$

$-4.97857500$

$-5.48608300$

$-4.76223600$

$-2.48569200$

$-3.39356000$

$-1.62971200$
2.52903600

1. 34748100

3. 09974600

3. 81716300

2.29824700

3. 62643200

3.26303600

$-2.16840000$

$-1.98200400$
0.00343000
0.42500700
0.20859900
$-0.58072900$
0.23974100
1.16980700
$-0.54714700$
0.17398300
$-1.09914700$

\section{p-TS9}

C

C

C

C

C

C

$\mathrm{H}$

$\mathrm{H}$

$\mathrm{H}$

$\mathrm{H}$

C

$\mathrm{H}$

$\mathrm{H}$

C

$\mathrm{H}$

$\mathrm{H}$

C

O

$\mathrm{N}$

C

C

C

C

C

$\mathrm{H}$

C

$\mathrm{H}$

$\mathrm{H}$

$\mathrm{H}$

C

$\mathrm{H}$

$\mathrm{H}$
0.56791900

0.01916700

$-1.30420400$

$-2.13030300$

$-1.58837100$

$-0.26989600$

1.76777500

$-1.72687600$

$-2.22244100$

0.12394400

$-3.60645900$

$-3.80138100$

$-3.98519200$

$-4.38283800$

$-5.45964500$

$-4.17707600$

$-4.05258700$

$-4.12129100$

$-3.67496100$

$-3.59028100$

$-2.36106700$

$-4.77878300$

$-2.36821100$

$-4.76923300$

$-5.71319800$

$-3.55695700$

$-1.41970400$

$-5.70347000$

$-3.53598500$

$-3.41729600$

$-4.34656500$

$-2.69374800$
2.03385500

2. 05102700

2. 41094800

2.76982700

2.80499600

2.43370200

2. 64262900

2. 37293500

3.09728700

2.43542900

3. 00205500

3. 37895100

3.73635500

1. 67758100

1.87990200

1.25643300

0.71057500

1.08752400

$-0.57333300$

$-1.08587000$

$-1.21863800$

$-1.43588500$

$-1.67857500$

$-1.90455200$

$-1.32171600$

$-2.02564400$

$-1.75687100$

$-2.17073000$

$-2.38682900$

$-1.47302100$

$-1.66293000$

$-1.01599600$
0.33030600

$-0.97491900$

$-1.19979000$

$-0.12260100$

1.17380200

1.39838500

0.35187000

$-2.19954300$

2.00814800

2.41166500

$-0.33502900$

$-1.34311600$

0.38514800

$-0.15454000$

$-0.21275800$

0.83161000

$-1.29339200$

$-2.46175400$

$-0.98976400$

0.34281600

1.02515100

0.99437400

2.35072600

2. 30726200

0.45279500

2. 98841500

2.87035000

2.79380500

4.01259000

$-2.12028700$

$-2.66908100$

$-2.79675300$ 
$\mathrm{H}$

C

O

$\mathrm{O}$

$\mathrm{Pd}$

$\mathrm{H}$

C

O

O

C

$\mathrm{H}$

$\mathrm{H}$

$\mathrm{H}$

C

O

O

C

$\mathrm{H}$

$\mathrm{H}$

$\mathrm{H}$

Ag
$-3.00982400$

$-1.02753400$

$-0.11711800$

$-0.89934600$

1.71460400

0.64610500

3.79041600

3.57281400

2. 89757900

5.22511000

5.28998300

5.53722500

5.87939700

3. 45610700

2. 82190400

3.17433600

4.69015700

4.94509100

5.52402000

4.53404000

1.15958100
$-2.40681500$

$-0.91147800$

$-0.55131300$

$-1.07355900$

0.29605800

1. 77082200

2.33007700

1.08629400

3.23302000

2. 77077200

3.44343900

3.33478200

1.90930600

$-2.12115400$

$-1.48296500$

$-2.17951400$

$-2.87313900$

$-3.67287300$

$-2.16264900$

$-3.27002500$

$-1.48078400$
$-1.73579600$

0.39612500

1.21514600

$-0.84900200$

0.72000300

$-1.81835900$

0.23158800

0.35215800

0.26279000

0.04641500

$-0.81351900$

0.93182600

$-0.09100800$

0.20498400

1.10451700

$-1.02965300$

0.67314900

$-0.02471800$

0.71834600

1.67903700

$-1.49501200$

\section{$o_{1}$-TS9}

C

C

C

C

C

C

$\mathrm{H}$

C

$\mathrm{H}$

$\mathrm{H}$

C

$\mathrm{H}$

$\mathrm{H}$

C

O

$\mathrm{N}$

C

C

C

C
$-0.29444200$

0.66646000

0.38980000

$-0.88049000$

$-1.83350900$

$-1.55580700$

1.39269500

$-1.19716700$

$-1.42799100$

$-0.32135800$

$-2.38914300$

$-3.18655400$

$-2.80666800$

$-1.95937500$

$-1.04503400$

$-2.70432200$

$-3.47282600$

$-2.89274500$

$-4.86599700$

$-3.73472000$
1.25217900

3.38189400

1.22719000

1.71025300

2.28346300

2.31538200

1.78623100

2.48965100

2.93292200

3.98818700

2. 91473200

2. 31479400

2.02008500

3. 07857500

1.17952800

1. 34751100

0.01512800

$-0.37296400$

$-0.85281100$

$-0.31263500$

$-1.24188700$
2.37781200

1.07461100

0.81070900

1.83672700

3.09751000

0.59907300

$-0.52132100$

$-0.32420100$

$-1.17630800$

$-1.28528700$

$-0.59940600$

$-1.95276200$

$-2.21233100$

$-3.00897700$

$-2.19000500$

$-1.04229700$

0.15618100

$-1.15103500$

1.20872900 


\begin{tabular}{|c|c|c|c|}
\hline $\mathrm{C}$ & -5.68937000 & -0.69653400 & -0.09320900 \\
\hline $\mathrm{H}$ & -5.28831200 & 0.05088500 & -2.08295400 \\
\hline $\mathrm{C}$ & -5.11974500 & -1.16067900 & 1.09407400 \\
\hline $\mathrm{H}$ & -3.27273300 & -1.60484000 & 2.11990000 \\
\hline $\mathrm{H}$ & -6.76909400 & -0.63280500 & -0.19704000 \\
\hline $\mathrm{H}$ & -5.75109700 & -1.46140000 & 1.92536300 \\
\hline $\mathrm{C}$ & -2.24732400 & -1.07571000 & -3.05741700 \\
\hline $\mathrm{H}$ & -1.37759700 & -1.57981200 & -2.62030800 \\
\hline $\mathrm{H}$ & -1.95582300 & -0.65672600 & -4.02099900 \\
\hline $\mathrm{H}$ & -3.06639100 & -1.78711800 & -3.19068600 \\
\hline C & -1.40801000 & -0.95349200 & 0.37956400 \\
\hline 0 & -0.67163000 & -0.40794300 & -0.50901800 \\
\hline O & -1.00156700 & -1.56514700 & 1.40536800 \\
\hline $\mathrm{H}$ & -2.80802600 & 2.76036100 & 1.64805100 \\
\hline $\mathrm{H}$ & -2.32162600 & 1.80808200 & 3.86875900 \\
\hline $\mathrm{H}$ & -0.07250800 & 0.86347900 & 4.37181100 \\
\hline $\mathrm{H}$ & 1.65771900 & 0.83234000 & 2.59145200 \\
\hline $\mathrm{Pd}$ & 1.23146600 & 0.36277300 & -0.34672700 \\
\hline $\mathrm{C}$ & 2.84660600 & -2.03465800 & -1.19402900 \\
\hline $\mathrm{O}$ & 2.08611600 & -1.10577800 & -1.61379700 \\
\hline 0 & 2.88538500 & -2.52446100 & -0.02497200 \\
\hline $\mathrm{C}$ & 3.82401100 & -2.59234800 & -2.21524000 \\
\hline $\mathrm{H}$ & 4.69722500 & -1.93028700 & -2.24970500 \\
\hline $\mathrm{H}$ & 3.37027900 & -2.59872300 & -3.20908000 \\
\hline $\mathrm{H}$ & 4.15139200 & -3.59410400 & -1.93053500 \\
\hline $\mathrm{C}$ & 3.29707600 & 2.41369100 & -0.12155100 \\
\hline 0 & 3.11644700 & 1.17823800 & -0.34858900 \\
\hline $\mathrm{O}$ & 2.40346400 & 3.21027100 & 0.30761700 \\
\hline $\mathrm{C}$ & 4.66968600 & 2.97621900 & -0.40927200 \\
\hline $\mathrm{H}$ & 4.66610300 & 3.38262200 & -1.42738100 \\
\hline $\mathrm{H}$ & 5.42334300 & 2.18903000 & -0.35211100 \\
\hline T & 4.89764000 & 3.78869300 & 0.28364000 \\
\hline Ac & 1.13508400 & -2.10137700 & 1.20967900 \\
\hline
\end{tabular}

\section{m-TS10}

$\mathrm{C}$
$\mathrm{C}$
$\mathrm{C}$
$\mathrm{C}$
$\mathrm{C}$
$\mathrm{C}$
$\mathrm{H}$
$\mathrm{H}$

2.34071200

1.83103500

2. 26261200

3. 12324100

3.59947400

3.23229200

2.03767400

1.81792500
$-1.77599000$

$-0.45795800$

0.43672200

0.03090200

$-1.28855100$

$-2.18284900$

$-2.47232200$

0.08438200 


-0.31669900
1.46993900
-0.32963400
1.86243400
1.50584300
2.39176700
2.86726400
3.78255500
3.14888000
2.28938900
1.62278000
2.50806000
3.26422800
2.65893100
4.65116300
3.44764400
5.42433700
5.11433100
4.82597900
2.96418600
6.49928600
5.42866500
1.77911800
1.94664000
0.70292300
2.16294900
1.18127600
0.47532700
0.72286200
-1.62855700
-1.67747300
-1.55804000
-1.80676000
-0.96534300
-1.81821200
-2.72314600
-4.29683300
-3.66460200
-5.77212500
-6.31393200
-6.16304700
-5.89990300
-3.78112300
-1.68882400

$-0.31669900$

1.88143400

1.45357700

4.27038600

$-1.62188200$

3.63112000

$-3.19411100$

3. 44622000

0.95118300

3. 66270100

1.96262000

4.33342500

0.58542400

2.27347900

1.03936000

2. 61695400

1.53991800

1.94218600

0.03647600

1.13188900

1.87835000

1.36906400

2.88126000

$-0.17071400$

1.48439500

$-0.55596200$

0.34236200

$-1.21340500$

$-0.75102700$

0.33570300

$-1.71095700$

$-1.79598000$

$-0.73507500$

1.19233700

0.10323600

$-1.79768600$

$-2.61366000$

$-0.72356200$

$-0.66984400$

$-2.62045700$

2. 23300300

3. 30176200

2. 04022500

1.93195500

$-0.84011700$

$-0.45294800$

$-1.35290000$

$-2.44331600$

$-0.38715600$

0.64426500

1.71373200

0.53478000

$-0.04184900$

1.52934500

$-0.00612800$

0.20295300

$-0.25432000$

$-0.26735200$

0.47474400

1.46742600

0.23383300

$-0.29645800$

$-0.09135100$

0.35024200

1.54477400

1.86487100

0.46635700

$-2.39018600$ 


\section{p-TS10}

C

C

C

C

C

C

$\mathrm{H}$

$\mathrm{H}$

$\mathrm{H}$

$\mathrm{H}$

C

$\mathrm{H}$

$\mathrm{H}$

C

$\mathrm{H}$

$\mathrm{H}$

C

$\mathrm{O}$

$\mathrm{N}$

C

C

C

C

C

$\mathrm{H}$

C

$\mathrm{H}$

$\mathrm{H}$

$\mathrm{H}$

C

$\mathrm{H}$

$\mathrm{H}$

$\mathrm{H}$

C

O

$\mathrm{O}$

$\mathrm{Pd}$

$\mathrm{H}$

C

O
1.10468200

0.60993100

$-0.65868400$

$-1.48227600$

$-0.96891900$

0.30263500

2. 44192200

$-1.05229400$

$-1.59285300$

0.67302300

$-2.94592300$

$-3.11338100$

$-3.30863600$

$-3.77735100$

$-4.83467100$

$-3.68732900$

$-3.29274900$

$-3.18695200$

$-2.85420200$

$-3.22556300$

$-2.29751200$

$-4.58280900$

$-2.75906200$

$-5.01736500$

$-5.29939200$

$-4.10395300$

$-2.03678900$

$-6.07434000$

$-4.43625600$

$-2.11874600$

$-1.97527900$

$-2.69368900$

$-1.14615100$

$-0.82482800$

$-0.29023400$

$-0.17018000$

1.79107200

1. 21808600

4.29928600

3.79318100
$-1.86619500$

$-2.34516700$

$-2.90555400$

$-2.98796200$

$-2.59963400$

$-2.04643700$

$-2.16038600$

$-3.21288500$

$-2.69439000$

$-1.71562600$

$-3.33250400$

$-3.93991900$

$-3.89265600$

$-2.02804300$

$-2.28285100$

$-1.44394200$

$-1.25734500$

$-1.82759900$

0.04883400

0.89050900

1.71009100

0.98369800

2. 60262600

1.83532200

0.39117500

2. 65875400

3.23606700

1.87759100

3.34229400

0.60984100

1.67933100

0.44861600

0.12233500

1. 62635900

0.55809700

2.72266000

0.12617300

$-2.24015700$

$-1.35067100$

$-0.20387100$
$-0.40311000$

0.82980400

0.92575300

$-0.20853900$

$-1.45636300$

$-1.55285500$

$-0.57877600$

1.88947000

$-2.34316300$

$-2.52061100$

$-0.06092700$

0.83253200

$-0.93062100$

0.06836400

0.20802300

$-0.84981000$

1.29730900

2.37762000

1.14835500

0.07137300

$-0.61416100$

$-0.27352400$

$-1.59675700$

$-1.28558800$

0.28637500

$-1.94661800$

$-2.09905400$

$-1.53310700$

$-2.72177300$

2. 29317000

2.13458700

3.20786700

2.39009000

$-0.41188100$

$-0.04863900$

$-0.69063300$

$-0.21265100$

1.72564200

$-0.60104900$

$-0.42720800$ 
$\mathrm{O}$
$\mathrm{C}$
$\mathrm{H}$
$\mathrm{H}$
$\mathrm{H}$
$\mathrm{C}$
$\mathrm{O}$
$\mathrm{O}$
$\mathrm{C}$
$\mathrm{H}$
$\mathrm{H}$
$\mathrm{H}$
$\mathrm{H}$

\section{$o_{1}$-TS10}

C

C

C

C

C

C

$\mathrm{H}$

C

$\mathrm{H}$

$\mathrm{H}$

C

$\mathrm{H}$

$\mathrm{H}$

C

$\mathrm{O}$

$\mathrm{N}$

C

C

C

C

C

$\mathrm{H}$

C

$\mathrm{H}$

$\mathrm{H}$

$\mathrm{H}$

C

$\mathrm{H}$
3.64145400

5.80568100

6.09315600

6.24382300

6.17423200

2. 57936200

2. 20607100

2.70285400

2. 83669000

1. 93128100

3. 13516200

3. 62074900

0.84500200
$-2.43723800$

$-1.44146200$

$-2.10444200$

$-0.45127200$

$-1.88316100$

2. 57087000

2.18977400

1.81144200

4.06689700

4. 61973100

4.33302800

4.35304900

2.60262100
$-0.69756500$

$-0.67866200$

$-1.49874900$

$-0.80741500$

0.25380400

1. 16231000

$-0.05874700$

2.10896900

1.25568500

0.97909400

2. 27113800

0.54697500

$-0.49182800$
1.36168600

1.89733200

1.38608900

0.32745000

$-0.18612600$

0.30983700

2.34139900

$-0.22938100$

$-0.30255100$

0.45736300

$-1.62554900$

$-2.05328500$

$-2.32385000$

$-1.60794900$

$-0.74982200$

$-2.68037800$

$-3.49935800$

$-2.97091800$

$-4.82234100$

$-3.74476500$

$-5.60590200$

$-5.22189500$

$-5.06803600$

$-3.31775400$

$-6.63425000$

$-5.67686000$

$-2.79253100$

$-2.38454300$
$-3.17187500$

$-2.14002200$

$-1.83267900$

$-2.61635500$

$-3.66586700$

$-3.93074200$

$-1.69103400$

$-2.36908200$

$-3.33215000$

$-1.74814500$

$-1.69552300$

$-1.70050300$

$-2.26135500$

$-0.28244000$

0.08014200

0.53711300

0.32895300

0.59647500

$-0.09820800$

0.40470400

$-0.27016000$

$-0.30031400$

$-0.02327000$

0.59346100

$-0.60610300$

$-0.16531300$

1.85014300

2.63672400
$-2.47145700$

$-1.70918500$

$-0.42437000$

0.10239100

$-0.66847500$

$-1.94534300$

0.48563700

1.48746300

2.00598700

2.06891900

1.48077900

0.47712400

2.11191800

2.06841400

2.86228500

1.74112700

0.58468500

$-0.68972000$

0.70390400

$-1.83326400$

$-0.43934900$

1.69327400

$-1.70453700$

$-2.81370000$

$-0.34212600$

$-2.59252200$

2. 38802100

1.74423500 


$\begin{array}{lrrr}\mathrm{H} & -2.22991600 & 1.82103500 & 3.32064600 \\ \mathrm{H} & -3.84694800 & 2.06148000 & 2.59001700 \\ \mathrm{C} & -1.53423500 & 1.01566000 & -0.73336100 \\ \mathrm{O} & -0.66921000 & 0.13048600 & -0.90067500 \\ \mathrm{O} & -1.30420200 & 2.27099900 & -0.51681700 \\ \mathrm{H} & -0.98502800 & -4.28367400 & -0.26306600 \\ \mathrm{H} & -0.11403300 & -4.74537300 & -2.52766700 \\ \mathrm{H} & 1.75767000 & -3.39280000 & -3.45879800 \\ \mathrm{H} & 2.72824000 & -1.55636800 & -2.09901900 \\ \mathrm{Pd} & 1.33306100 & 0.28744200 & -0.21130900 \\ \mathrm{C} & 1.89757700 & 3.00064300 & -1.17460200 \\ \mathrm{O} & 1.14922800 & 2.39446900 & -0.24678500 \\ \mathrm{O} & 2.64571500 & 2.41627400 & -1.93658200 \\ \mathrm{C} & 1.71689100 & 4.51020900 & -1.17115600 \\ \mathrm{H} & 2.33354100 & 4.96203000 & -1.94998700 \\ \mathrm{H} & 1.99520700 & 4.91270000 & -0.19158600 \\ \mathrm{H} & 0.66379600 & 4.76436600 & -1.33975800 \\ \mathrm{C} & 3.75550200 & -0.41572400 & 1.25584300 \\ \mathrm{O} & 3.17740900 & 0.52270300 & 0.62447400 \\ \mathrm{O} & 3.35639800 & -1.61884100 & 1.30107000 \\ \mathrm{C} & 5.00093300 & -0.04514400 & 2.02979500 \\ \mathrm{H} & 5.69662500 & -0.88649300 & 2.04661200 \\ \mathrm{H} & 4.70763600 & 0.18106800 & 3.06142400 \\ \mathrm{H} & 5.46739600 & 0.84111200 & 1.59672300 \\ \mathrm{H} & -0.25747000 & 2.44549900 & -0.40655800\end{array}$

